Measurement of the $Z$ boson transverse momentum distribution at the Tevatron

\author{
$\mathrm{Ng}$, Johnny Shing Tung, Ph.D. \\ Harvard University, 1991
}

Copyright (C1991 by Ng, Johnny Shing Tung. All rights reserved.

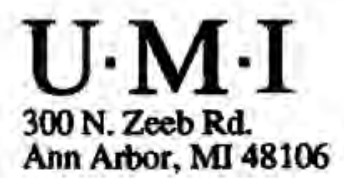





\section{HARVARD UNIVERSITY}

THE GRADUATE SCHOOL OF ARTS AND SCIENCES

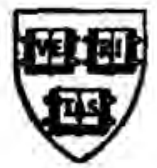

\section{THESIS ACCEPTANCE CERTIFICATE (To be placed in Original Copy)}

The undersigned, appointed by the

Division

Department of Physics

Committee

have examined a thesis entitled

"Measurement of the $\mathrm{Z}$ Boson Transverse Momentum Distribution at the Tevatron"

presented by Johnny Shing Tung $\mathrm{Ng}$

candidate for the degree of Doctor of Philosophy and hereby certify that it is worthy of acceptance.

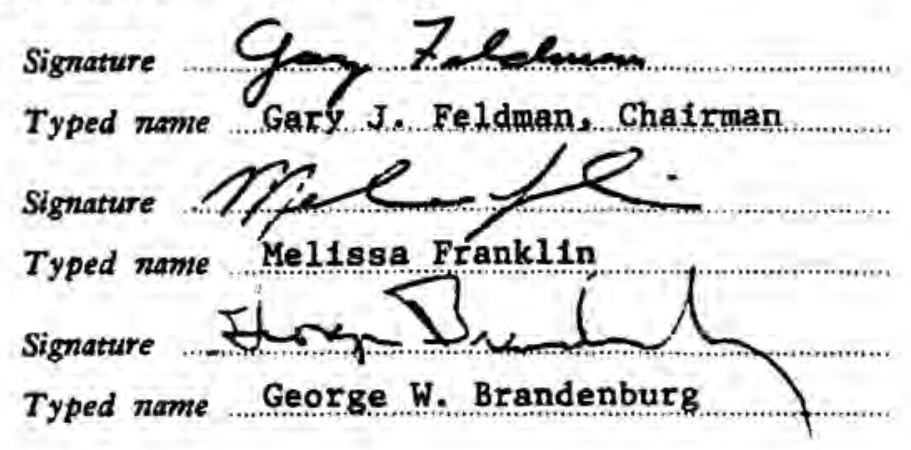

Date ..... May 21, 1991 



\title{
Measurement of the $Z$ Boson Transverse Momentum Distribution at the Tevatron
}

\author{
A thesis presented \\ by \\ Johnny Shing Tung Ng \\ to \\ The Department of Physics \\ in partial fulfillment of the requirements \\ for the degree of \\ Doctor of Philosophy \\ in the subject of \\ Physics \\ Harvard University
}

Cambridge, Massachusetts

June 1991 
Copyright (c) 1991 by Johnny Shing Tung Ng All Rights Reserved 


\begin{abstract}
We have measured the $Z$ boson production differential cross section as a function of transverse momentum using $Z \rightarrow e e$ and $Z \rightarrow \mu \mu$ decays in $\bar{p} p$ collisions at $\sqrt{s}=1.8$ TeV with the Collider Detector at Fermilab. Comparison with Standard Model predictions shows good agreement over the range $0<p_{T}<160 \mathrm{GeV} / c$ available from this data sample.
\end{abstract}




\section{Contents}

List of Tables $\quad$ v

List of Figures $\quad$ vi

Acknowledgements $\quad$ x

1 Introduction and Theoretical Orientation 1

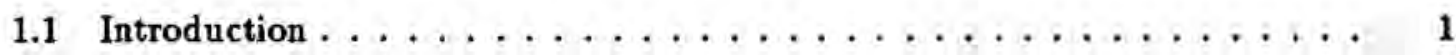

1.2 The Standard Model $\ldots \ldots \ldots \ldots \ldots \ldots \ldots \ldots \ldots \ldots$

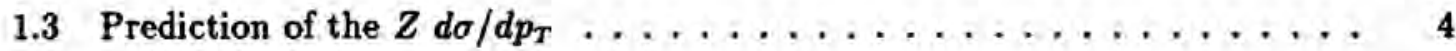

2 Experimental Apparatus $\quad 11$

2.1 The Fermilab Tevatron Accelerator $\ldots \ldots \ldots \ldots \ldots \ldots$

2.2 The Collider Detector at Fermilab (CDF) $\ldots \ldots \ldots \ldots \ldots \ldots$

2.2 .1 Tracking Devices ...................... 17

2.2 .2 Calorimeters ........................ 19

2.2 .3 Muon Detector . . . . . . . . . . . . . . . . 24

2.2.4 The Data Acquisition System . . . . . . . . . . . . 25

3 Data Collection and Reconstruction 29

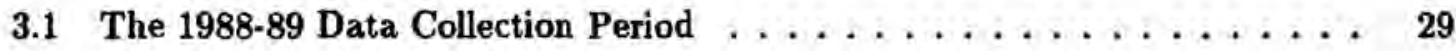

3.2 Data Reconstruction .................... 30

3.2 .1 Tracking . . . . . . . . . . . . . . . . . 32 
3.2 .2 The Electrons . . . . . . . . . . . . . . . . . 40

3.2.3 The Muons $\ldots \ldots \ldots \ldots \ldots \ldots \ldots \ldots \ldots \ldots$

3.2.4 Jet Clustering . . . . . . . . . . . . . . . 44

3.3 The Data Sample . . . . . . . . . . . . . . . 45

3.4 The Integrated Luminosity $\ldots \ldots \ldots \ldots \ldots \ldots \ldots \ldots \ldots$

4 Event Selection $\quad 48$

4.1 Electron Selection $\ldots \ldots \ldots \ldots \ldots \ldots \ldots \ldots \ldots \ldots \ldots$

4.1.1 Electron Fiducial Region $\ldots \ldots \ldots \ldots \ldots \ldots$

4.1 .2 Electron Identification Cuts . . . . . . . . . . . . 49

4.1.3 Electron Identification Cut Efficiency $\ldots \ldots \ldots \ldots \ldots$

4.2 Muon Selection ..................... 62

4.2 .1 Muon Fiducial Region . . . . . . . . . . . . . 62

4.2.2 Muon Identification Cuts . . . . . . . . . . . . 64

4.2.3 Muon Identification Cut Efficiency . . . . . . . . . . 67

4.3 Systematics in the Lepton Efficiencies $\ldots \ldots \ldots \ldots \ldots$

4.3.1 Isolation Cut Efficiencies: Muons vs. Electrons . . . . . . . . . . 69

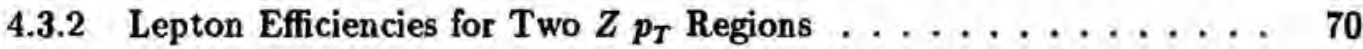

4.4 The Trigger Requirement $\ldots \ldots \ldots \ldots \ldots \ldots \ldots \ldots \ldots$

4.4.1 Electron Trigger Efficiency $\ldots \ldots \ldots \ldots \ldots \ldots$

4.4 .2 Muon Trigger Efficiency . . . . . . . . . . . . 73

4.5 The Selection of $Z \rightarrow e e$ and $Z \rightarrow \mu \mu$ Events $\ldots \ldots \ldots \ldots \ldots$

5 Event Properties of the Z Data Sample $\quad 78$

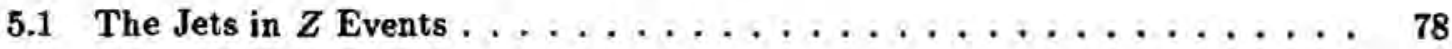

5.2 The Missing $E_{T}$ in $Z$ Events $\ldots \ldots \ldots \ldots \ldots \ldots \ldots \ldots$

5.3 Theoretical Expectations. . . . . . . . . . . . . . . 84

5.3.1 The Papageno Monte Carlo Event Generator . . . . . . . . . . . 84 
5.3.2 The Parton Fragmentation Simulation . . . . . . . . . . . . 84

5.3.3 The Detector Simulation . . . . . . . . . . . . . 85

5.4 Jet Energy Corrections . . . . . . . . . . . . . . . 86

5.5 Jet Selection Efficiency . . . . . . . . . . . . . . . 88

5.6 Comparison of Data with QCD Expectations . . . . . . . . 88

5.6.1 Jet Production . . . . . . . . . . . . . . . . 89

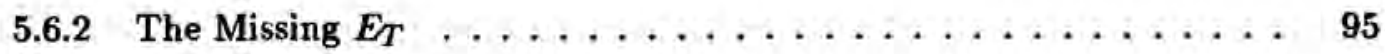

6 Measurement of the $Z d \sigma / d p_{T} \quad 98$

6.1 The Physics and Detector Models . . . . . . . . . . . . . . 98

6.1.1 The ISAJET Monte Carlo Event Generator . . . . . . . . . . 98

6.1.2 The Simple Detector Simulation . . . . . . . . . . 99

6.1.3 Monte Carlo Simulation vs. Raw Data . . . . . . . . . . . . . 101

6.2 The Acceptance Correction $\ldots \ldots \ldots \ldots \ldots \ldots \ldots \ldots$

6.2.1 Combining Data Samples with Different Acceptances. . . . . . . 107

6.3 The Correction for Resolution Smearing . . . . . . . . . . 108

6.4 Background Estimate $\ldots \ldots \ldots \ldots \ldots \ldots \ldots \ldots$

6.5 Correction for the Drell-Yan Continuum . . . . . . . . . . 117

7 Results and Conclusions $\quad 121$

7.1 The Procedure for Obtaining $d \sigma / d p_{T} \ldots \ldots \ldots \ldots \ldots \ldots \ldots \ldots$

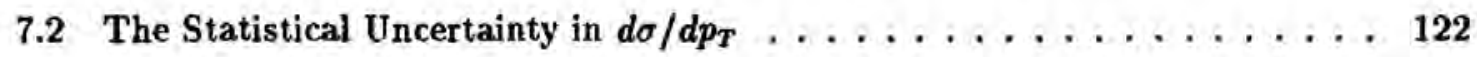

7.3 The Sources Of Systematic Uncertainties . . . . . . . . . . . . . 123

7.4 The Treatment of Correlated Uncertainties . . . . . . . . . . 125

7.4.1 Statistical Uncertainties Only . . . . . . . . . . . 126

7.4.2 Including Systematic Uncertainties . . . . . . . . . . 127

7.5 Results of the $Z p_{T}$ Distribution $\ldots \ldots \ldots \ldots \ldots \ldots \ldots$

$7,5.1$ Comparison With QCD Prediction ............. 131 
7.5.2 Comparison With the $W p_{T}$ Distribution $\ldots \ldots \ldots \ldots$

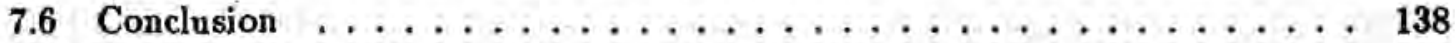

Bibliography 139 


\section{List of Tables}

2.1 Typical beam parameters of the Tevatron. . . . . . . . . . 11

3.1 Parameters in the offline EM clustering algorithm $\ldots \ldots \ldots \ldots \ldots \ldots$

3.2 Parameters in the offline Jet clustering algorithm $\ldots \ldots \ldots \ldots \ldots$

4.1 The electron fiducial volume . . . . . . . . . . . . . . . 49

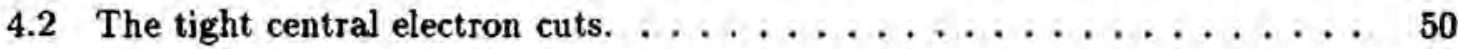

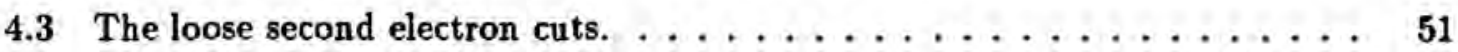

4.4 The isolated electron identification cut efficiency measured from $Z$ electrons. 58

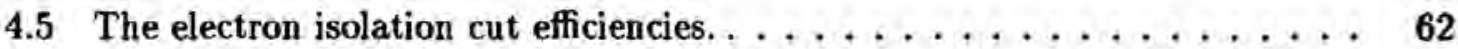

4.6 The total electron selection efficiency. $\ldots \ldots \ldots \ldots \ldots \ldots \ldots$

4.7 Fiducial region of the central muon drift chamber. . . . . . . . . 64

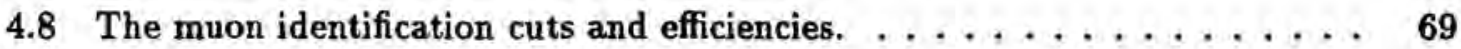

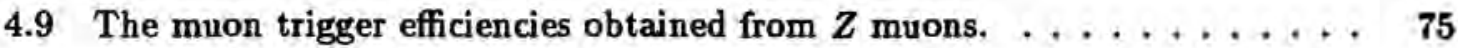

5.1 The $Z$ events with high jet multiplicity $\ldots \ldots \ldots \ldots \ldots \ldots \ldots$

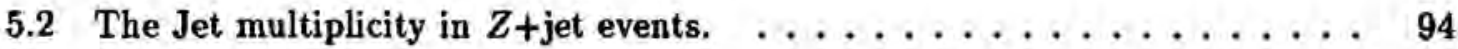

6.1 The smearing matrix $C_{i j} \ldots \ldots \ldots \ldots \ldots \ldots \ldots \ldots \ldots \ldots \ldots \ldots$

7.1 The percentage uncertainties broken down for each source. . . . . . . . 129

7.2 The correlation matrix. $\ldots \ldots \ldots \ldots \ldots \ldots \ldots \ldots \ldots \ldots \ldots$

7.3 Summary of the results and uncertainties. . . . . . . . . . . 131

7.4 The fit parameters of parametrizations of the $W$ and the $Z p_{T}$ spectra. . . 132 


\section{List of Figures}

1.1 Some diagrams that contribute to hadronic a) 0-jet, b) 1-jet, and c) 2-jet

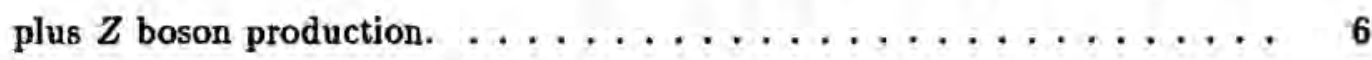

1.2 The $d \sigma / d p_{T}$ results calculated using the resummation, perturbative, and matching methods. .................... 8

1.3 The next-to-leading order QCD prediction of $d \sigma / d p_{T}$ shown as a band... . 10

2.1 General lay-out of the Fermilab a)Tevatron accelerator, and the b)antiproton source. .............................. 12

2.2 Flow chart illustrating the Tevatron operation cycle. . . . . . . . 13

2.3 The CDF detector: a perspective view of the various components showing nearly complete $4 \pi$ coverage. $\ldots \ldots \ldots \ldots \ldots \ldots \ldots$

2.4 The CDF detector: a cut-away view showing symmetry about the beam line; the other half is symmetric about the midplane. . . . . . . . . 16

2.5 The CTC: a) End view showing 9 superlayers. b) Trajectories in a drift cell; the arrow indicates the radial direction. $\ldots \ldots \ldots \ldots \ldots$

2.6 The calorimeter $\eta-\phi$ segmentation. . . . . . . . . . . 20

2.7 The light gathering layout of one CEM module. . . . . . . . . . 21

2.8 The Central Muon drift chamber system. . . . . . . . . . 26

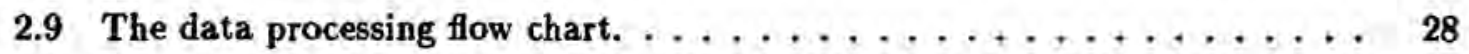

3.1 The CDF and Tevatron performance. . . . . . . . . . 30 
3.2 A $Z \rightarrow e e$ event observed in the CDF detector: the "lego" view of calorimeter transverse energy depositions in the $\eta \phi$ geometry. . . . . . . . .

3.3 The $Z$ event shown in the previous plot viewed along the beam axis in the

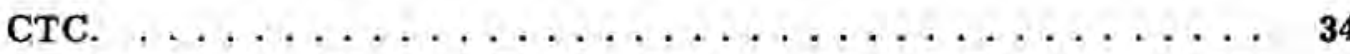

3.4 The CEM tower energy response function in local $x$ and $z$ coordinates. . . 41

4.1 The distribution of electron isolation for each detector region. . . . . . . 54

4.2 The distributions of variables used for tight CEM electron cuts from a sample

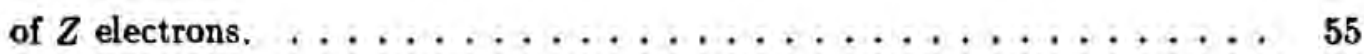

4.3 The distributions of cut variables used to identify the second electron in a $Z$

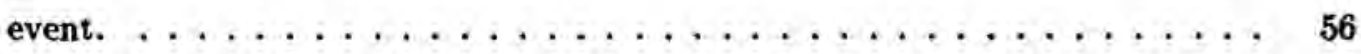

4.4 Ilustration of the method used for finding the isolation cut efficiency. . . 59

4.5 The distribution of equivalent electron isolation. . . . . . . . 61

4.6 The muon detection efficiency as a function of $z$ position. . . . . . . 63

4.7 The muon and pion energy spectra measured in the central calorimeters at the test beam. ......................... 65

4.8 The distributions of cut variables used in CMUO muon identification. . . . 66

4.9 The distributions of cut variables used in CMIO muon identification. . . . 68

4.10 The efficiency of isolation cut for each $\phi$-cone for central muons and electrons. 70

4.11 The efficiencies for two $Z p_{T}$ regions for central muons compared with central

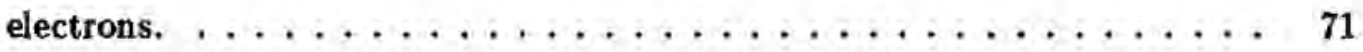

4.12 The efficiencies for two $Z p_{T}$ regions for FEM and PEM electrons. . . . . 72

4.13 The mass distributions of $\mu \mu$ and $e e$ pairs, including low mass pairs. . . . 77

4.14 The observed $p_{T}$ distributions of the $Z \rightarrow e e$ and $Z \rightarrow \mu \mu$ candidates. . . 77

5.1 The $Z p_{T}$ distributions for events with and without jets. . . . . 80

5.2 The distributions of some jet variables for $Z+1$-jet events. , . . . . 81

5.3 The distributions of some jet variables for the second jet in $Z+2$-jet events. 82 
5.4 The Missing $E_{T}$ distributions in $Z$ events. . . . . . . . . . 83

5.5 The $Z+1$-jet event properties compared with $\mathrm{QCD}$ predictions. . . . . 90

5.6 The $Z+2$-jet event properties compared with QCD predictions. . . . . 91

5.7 The jet-jet invariant mass distribution for $Z+2$-jet events. . . . . . 93

5.8 The Jet multiplicity in $Z$ events. . . . . . . . . . . . 93

5.9 The uncorrected Missing $E_{T}$ in $Z$ events compared with QCD expectation. $\quad 95$

5.10 The $\not_{T}$ distribution in $Z \rightarrow e \epsilon$ events before and after jet energy correction. 96

5.11 The $E_{T}$ recoiling against the $Z$ vs. the $Z p_{T} \ldots \ldots \ldots \ldots \ldots$

6.1 The observed (uncorrected) $Z p_{T}$ distributions. . . . . . . . 102

6.2 The lepton transverse momentum distributions. . . . . . . . . . . . . 102

6.3 The $Z$ rapidity distributions. . . . . . . . . . . . . 103

6.4 The lepton pseudo-rapidity distributions. $\ldots \ldots \ldots \ldots \ldots \ldots$

6.5 The invariant mass distributions of $\mu \mu$ and $e e$ pairs, including low mass pairs. 104

6.6 Two Monte Carlo $p_{T}$ spectra, one is the default, the other is adjusted to fit the raw data. . . . . . . . . . . . . . . . . 105

6.7 The combined $Z \rightarrow e e$ and $Z \rightarrow \mu \mu$ acceptance as a function of $p_{T} \ldots \ldots 108$

6.8 The $Z p_{T}$ resolution as a function of $p_{T} \ldots \ldots \ldots \ldots \ldots \ldots \ldots$

6.9 The "smearing fraction", or the probability that the $p_{T}$ in a given range is measured to be in some other range because of resolution smearing. . . . 112

6.10 The "unsmearing" mechanism. It shows how the content in each observed bin is to be distributed among the "unsmeared" bins. . . . . . . . 113

6.11 The results of consistency tests of the smearing and "unsmearing" matrices. 114

6.12 The effects of corrections on the $p_{T}$ spectrum. . . . . . . . . 115

6.13 Scatter plot of the maximum isolation of the two charged leptons vs. the invariant mass used in background estimate. . . . . . . . . 118

6.14 The distributions of maximum isolation for three background processes. . . 119 
7.1 The differential cross section for $Z$ production for the combined $Z \rightarrow e e$ and $Z \rightarrow \mu \mu$ samples. The next-to-leading order QCD calculation is shown as a band; the width of the band indicates the theoretical uncertainties. . . . . 133

7.2 The differential cross section for $Z$ production for the $Z \rightarrow e e$ and $Z \rightarrow \mu \mu$ samples separately. The next-to-leading order QCD calculation is shown as a band; the width of the band indicates the theoretical uncertainties. . . . .

7.3 The ratio of the $W$ and $Z p_{T}$ spectra to the nominal QCD prediction for the $Z$; the $W$ 's are normalized by the ratio of the measured $W$ and $Z$ total cross sections. The QCD prediction is shown as a band of solid (dotted) lines for

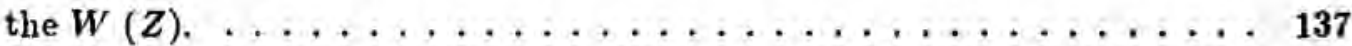




\section{Acknowledgements}

I thank the physicists and support staff of Fermilab and the members of the CDF collaboration for their dedicated effort that made this measurement possible. I am especially grateful to my friend and co-worker Ed Kearns for his generous help, and to Peter, David, Teruki, Brian, Craig and Marshall and many others. Peter Arnold and Russel Kauffman provided clear explanations about their calculations.

My thesis advisor Gary Feldman made available valuable time to work with me. His insights helped focus my attention on the relevant physics issues. Melissa Franklin tirelessly and critically followed each stage of this measurement. Her insistence on good work led to results of higher quality. George Brandenburg has been a constant source of support, despite the many changes in the group. His interest in this work was crucial, especially during the early stages. Gary, Melissa, and George also very carefully read several drafts of the thesis and made helpful comments and suggestions. Bob Cousins made some valuable suggestions, especially on the error calculation. Because of my work with these physicists, the otherwise simple academic exercise of writing a thesis turned out to be a great learning experience.

I also benefited from my associations with several other physicists over the years. Marjorie Shapiro and Roy Schwitters provided thoughtful guidance when I first joined the group. Sau Lan Wu gave me a valuable opportunity to work at CERN. Professor Chinowsky has been a source of wisdom ever since my undergraduate years at Berkeley.

It is known that spending too many hours in the lab is bad for one's health. I'm grateful to all my badminton friends for always being available for a good game that helped alleviate the tension and frustration associated with thesis work. Andy, Colin, Rowan, Ed, Peter, Rose, the staff at HEPL, and the street musicians and magicians in Harvard square all provided the necessary social (and sometimes anti-8ocial) distractions.

I have always relied on my family to provide the strength to carry on in difficult and 
sometimes seemingly impossible times. It is a pleasure to be able to share my accomplishment with my mother and father, brothers and sisters. Their encouragement and patience are appreciated.

"One's life has an end, but learning has no end," Many people give up learning knowing that they will never learn everything. Others only find dissatisfaction in not knowing more - my grandmother was such a person. She never had the chance to go to school. And yet, in her 70's, she was learning to read and write in Chinese. She told me once that she would have gotten at least a Ph.D. I'm sure she would have. This work is dedicated to the memory of my grandmother. 


\section{Chapter 1}

\section{Introduction and Theoretical Orientation}

\subsection{Introduction}

From the time when bombarding gold foils with $\alpha$ particles was used to probe matter at the distance scale of $10^{-6} \mathrm{~cm}$ to today's high energy hadron colliders that probe matter at the distance scale of $10^{-16} \mathrm{~cm}$, a wealth of experimental data on fundamental particles and forces has been accumulated. At the same time, the phenomenology of the Electromagnetic, Weak, and Strong interactions has been put on a firm theoretical foundation in terms of relativistic quantum field theories, also known as "gauge theories". Our knowledge of the basic constituents and forces of nature is embodied in a Standard Model of the electroweak and strong interactions.

The discovery in 1973 [1] of the neutral currents provided the first experimental evidence for the electroweak theory [2]. Further support followed in 1983 with the experimental observation [3] of the $W$ and $Z$ gauge bosons. Recent measurements of the $W$ mass [4], the $Z$ mass [5], and other electroweak parameters at the $Z$ resonance [6] have provided more stringent tests.

Precise tests of the strong interaction theory, Quantum Chromodynamics (QCD), are more difficult. Because of the large strong interaction coupling, the perturbative QCD calculations are valid only for large momentum transfer processes where the effective coupling becomes small. Also, QCD is a theory of confined quarks and gluons, whereas only hadrons 
are observed in the experiment. Nevertheless, the large number of experimental results obtained so far indicates that QCD is the best theory for the strong interaction. These tests include jet production in $e^{+} e^{-}$and $\bar{p} p$ collisions, lepton production in deep inelastic scattering processes, Drell-Yan processes, and $W / Z$ productions, among others. [7]

In this thesis, we carry out a study of the $Z$ production property in $\tilde{p} p$ collisions as a test of QCD. We present the result of a measurement of the differential cross section, as a function of the transverse momentum $\left(p_{T}\right)$, of $Z$ gauge bosons produced in protonantiproton collisions at a center-of-mass energy of $1.8 \mathrm{TeV}$. This measurement is based on data collected with the Collider Detector at Fermilab (CDF) during the 1988-89 run. The result is compared with the prediction of a next-to-leading order QCD calculation.[8]

In the rest of this Chapter, we describe the Standard Model and the calculation of the $Z p_{T}$ distribution. The experimental apparatus is described in Chapter 2. In Chapter 3, we discuss the procedure of reconstructing a data sample of $Z$ events consisting of electrons and muons from the raw data recorded by our instruments. The $Z$ event selection is detailed in Chapter 4. Some properties of these $Z$ events are examined in Chapter 5 . We describe the measurement of the $Z d \sigma / d p_{T}$ in Chapter 6. The result is presented in Chapter 7, along with comparison with theoretical calculations.

\subsection{The Standard Model}

Fermi's theory, in its V-A form with a four-fermion interaction, provides a good description of all low energy weak interaction data. Since typical weak interactions, such as $\beta$-decay, are slow and involve charge changes, the particle transmitting the weak force must be heavy and charged. At high energy, however, Fermi's theary predicts unphysically large cross sections. Also, the theory is not renormalizable - in higher order calculations, the singularities cannot be removed by absorbing them into the definition of physical quantities.

In an attempt to solve this problem, Yang and Mills developed a model with invariance under a local transformation of the gauge group SU(2). The requirement of gauge 
invariance ensures renormalizability. The yector particles that transmit the weak force, the gauge bosons, can interact with each other but are required to be massless like the photon. The bosons acquire mass via the Higgs mechanism which spontaneously breaks the gauge symmetry but preserves the renormalizability of the theory. In the Standard Model, the electromagnetic force is unified with the weak force. An additional symmetry under the $\mathrm{U}(1)$ gauge group is required. It results in four gauge bosons: two massive charged $W$ bosons, one massive neutral $Z$ boson, and the (massless) photon. The Fermi theory required only the charged $W$ bosons. The existence of the neutral $Z$ boson in the Standard Model led to the prediction of weak neutral current processes such as $\nu_{\mu} e^{-} \rightarrow \nu_{\mu} e^{-}$. The gauge boson masses and interactions can be determined by three parameters: the electromagnetic coupling $\alpha \approx 1 / 137$, the Fermi coupling $G_{F} \approx 1.166 \times 10^{-5} \mathrm{GeV}^{-2}$, and the weak mixing angle $\theta_{W}$. The value of $\sin ^{2} \theta_{W}$ has been measured in a variety of experiments, and the world average is $0.2259 \pm 0.0046$.[9]

In this thesis, we are interested in the production of $Z$ bosons in $\bar{p} p$ collisions as a test of the QCD description of strong interactions. Deep inelastic scattering experiments $e p \rightarrow e X$ showed that the proton structure functions depend approximately only on a dimensionless variable. This scaling behavior is interpreted as due to interactions of the probe, the virtual photon or the $Z$, with point-like constituents inside the proton. These point-like constituents, with electroweak couplings, are identified with the quarks. Applying momentum sum rules to the quark distributions reveals the existence of electroweak-neutral partons - these are identified as the giuons. QCD is a gauge theory with "color" SU(3) symmetry and it provides a field theoretical basis for the naive parton model. It describes the interactions between the quarks and the gluons, as well as among the gluons themselves. Because of the non-observation of free quarks, a long range strong interaction is desirable. The SU(3) gauge bosons, the gluons in this case, remain massless and there is no need for spontaneous symmetry breaking. QCD is asymptotically free. The effective strong coupling becomes small at short distances, so that perturbative calculations can be used to make 
quantitative predictions.

\subsection{Prediction of the $Z d \sigma / d p_{T}$}

The major ingredients in a QCD calculation for $Z$ production in $\bar{p} p$ collisions are the following:

1. The parton momentum distribution functions. The $Z$ is produced through interactions between the quarks and/or gluons, and information on the momenta of the colliding quarks or gluons are necessary for the calculation. The parton momentum distributions are obtained from measurements of various processes, including deep inelastic scattering of muons on nuclear targets. Because of gluon radiation, these distributions depend on the momentum scale $Q^{2}$ characteristic of the process. In QCD, the distributions measured from a given process at a given scale can be used for

any process at any scale. The distribution function $f_{a}\left(x_{a}, Q^{2}\right)$ gives the probability that a parton of type $a$ carries a fraction $x_{a}$ of the parent hadron's momentum.

2. The strong interaction coupling "constant". Perturbative predictions are computed as an expansion in the strong coupling "constant" $\alpha_{s}$. In evaluating the matrix elements, some procedure must be used to remove singularities. This "renormalization" procedure introduces a scale dependence, and the strong coupling is expressed as:

$$
a_{s}\left(Q^{2}\right)=\frac{12 \pi}{(33-2 f) \ln \left(Q^{2} / \Lambda_{Q C D}^{2}\right)},
$$

where $Q^{2}$ is a momentum scale, $\Lambda_{Q C D}$ is a parameter to be determined by experiment, usually at the same time the parton momentum distributions are determined, and $f$ is the number of quark flavors participating in the process.

3. The hard scattering cross section. The parton scattering cross section $\hat{\sigma}\left(x_{a}, \ldots, \alpha_{s}\left(Q^{2}\right)\right)$, for processes such as $q+\bar{q} \rightarrow Z$ and $q+g \rightarrow Z+q$, is calculated using perturbative methods. 
The differential cross section for the hadronic process, $\tilde{p} p \rightarrow Z+$ anything, is then expressed as:

$$
\frac{1}{p_{T}} \frac{d \sigma}{d p_{T}}=\sum_{a, b} C_{a, b} \int d x_{a} d x_{b} f_{a}\left(x_{a}, Q^{2}\right) f_{b}\left(x_{b}, Q^{2}\right) \frac{1}{p_{T}} \frac{d \hat{\sigma}}{d p_{T}}\left(x_{a}, x_{b}, \ldots, \alpha_{s}\left(Q^{2}\right)\right)
$$

where we sum over all parton types $a, b$ with color factor $C_{a, b}$. The scale in the parton distributions is identified with the scale in $\alpha_{s}$. The scale can be chosen among the physical scales for a given process. For example, for hadronic $Z$ production, the scales could be $Q^{2}=M_{Z}^{2}$ or $Q^{2}=p_{T}^{2}$. This ambiguity arises only because perturbative calculations are carried out to a finite order.

In the perturbative approach $[8,10,11]$, which is the way quantitative predictions can be extracted from $Q C D$, the calculation of the $Z d \sigma / d p_{T}$ can be expressed as:

$$
\frac{1}{p_{T}} \frac{d \sigma}{d p_{T}}=\alpha_{w} \alpha_{s}\left(u_{1}+u_{2} \alpha_{8}+u_{3} \alpha_{s}^{2}+\cdots\right)
$$

where $\alpha_{w}=\sqrt{2} G_{F} M_{W}^{2} / \pi$. Figure 1.1 shows the sample Feynman diagrams of processes that are included in the calculation. If all processes at all orders of $\alpha_{s}$ are calculated, the perturbation series converges and a finite result for $d \sigma / d p_{T}$ can be obtained. But, given the large number of processes involved, this is impossible with present day technology. An estimate, however, can be obtained by calculating the contributions at the first few orders of $\alpha_{s}$, assuming the contributions from higher orders are small. For large $p_{T}$, contributions from all processes up to $\mathcal{O}\left(\alpha_{s}^{2}\right)$ have been calculated, and a finite result obtained for $d \sigma / d p_{r} \cdot[10]$

The leading-logarithm approximation to Equation 1.3, including only the dominant terms at each order, has the form:

$$
\frac{1}{p_{T}} \frac{d \sigma}{d p_{T}} \approx \frac{\alpha_{w} \alpha_{s}}{p_{T}^{2}} \ln \left(\frac{Q^{2}}{p_{T}^{2}}\right)\left[v_{1}+v_{2} \alpha_{s} \ln ^{2}\left(\frac{Q^{2}}{p_{T}^{2}}\right)+v_{3} \alpha_{s}^{2} \ln ^{4}\left(\frac{Q^{2}}{p_{T}^{2}}\right)+\cdots\right],
$$

where $Q^{2}$ is the squared boson mass. Each factor of $\alpha_{3}$ is associated with the radiation of either a real or virtual gluon. Each gluon potentially has both mass and collinear infrared singularities. These singularities are rendered finite by introducing a lower cut off at $p_{T}$ in 

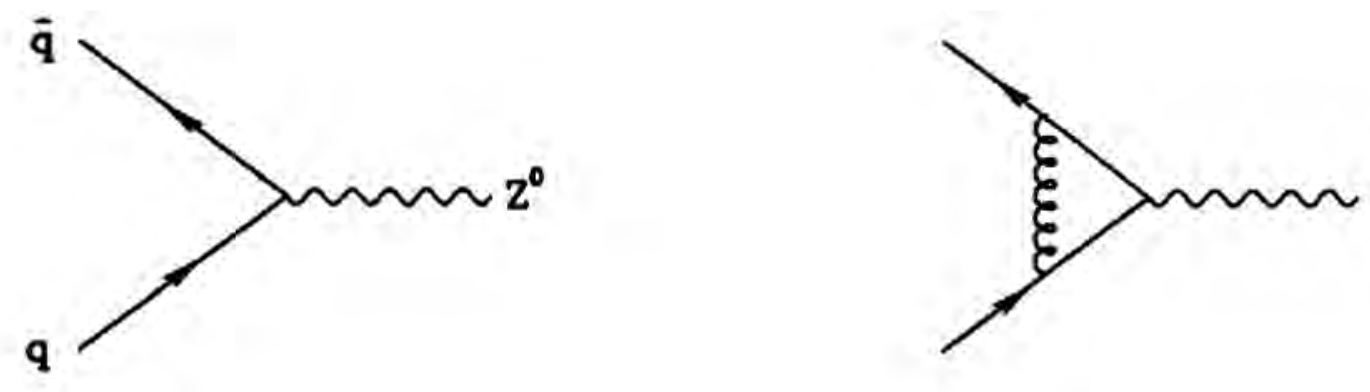

a)
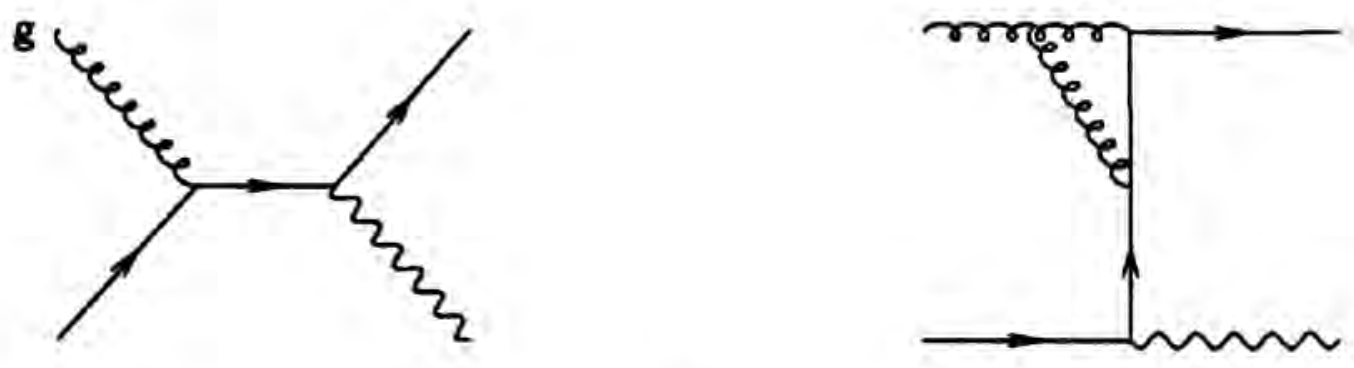

b)
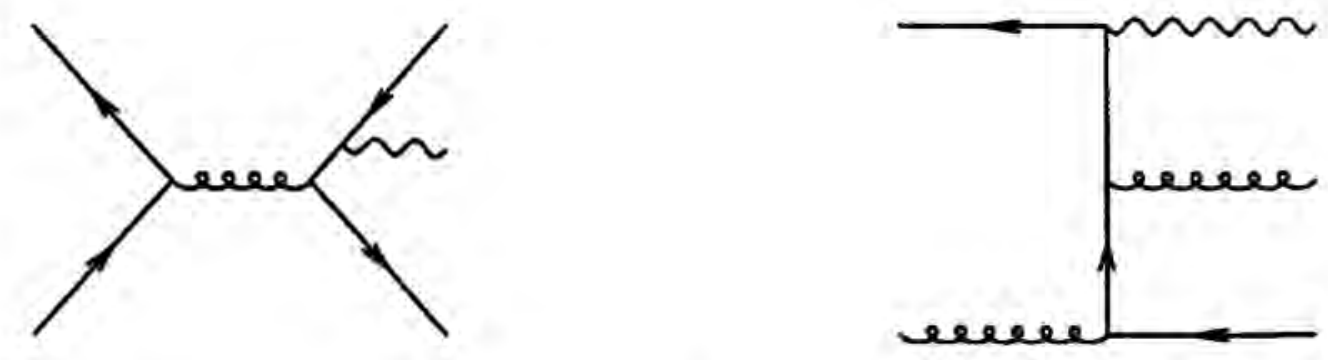

c)

Figure 1.1: Some diagrams that contribute to hadronic a) 0-jet, b) 1-jet, and c) 2-jet plus $Z$ boson production. 
the phase space integral. This results in the logarithmic term $\ln \left(Q^{2} / p_{T}^{2}\right)$ for each factor of $\alpha_{s}$. Note, now the convergence of the perturbation series is governed not by $\alpha_{s}$ alone, but by $\alpha_{s} \ln \left(Q^{2} / p_{T}^{2}\right)$. For small $p_{T}, \alpha_{g} \ln \left(Q^{2} / p_{T}^{2}\right)$ can be large even if $\alpha_{s}$ is small, and the finite order perturbative calculation is no longer valid. Also note that the overall factor of $1 / p_{T}^{2}$ leads to an unphysically large cross section at small $p_{r}$.

Fortunately, the series in Equation 1.4 can be summed. In fact, terms in addition to those in the leading-logarithmic approximation which are at least as singular as $1 / p_{T}^{2}$ as $p_{T} \rightarrow 0$ can be included in the sum. The expansion is organized as:

$$
\frac{1}{p_{T}} \frac{d \sigma}{d p_{T}} \approx \frac{\alpha_{w} \alpha_{s}}{p_{T}^{2}}\left(Z_{1}+Z_{2} \alpha_{s}+Z_{3} \alpha_{s}^{2}+\cdots\right)
$$

and the $Z_{n}$ 's now contain finite sums of $\alpha_{s} \ln \left(Q^{2} / p_{T}^{2}\right)$ terms. $Z_{1}$ has been completely cal. culated. Only part of $Z_{2}$ is available. The resummation result is finite as $p_{T} \rightarrow 0$, and is valid at next-to-leading order.[8] The procedure for summing these large logarithmic terms in the perturbation series is generally referred to as "soft gluon resummation".[12]

The result obtained using Equation 1.5 only contains contributions from terms which diverge as $1 / p_{T}^{2}$ as $p_{T} \rightarrow 0$, and is a good approximation for $p_{T} \ll Q^{2}$. The perturbative result is valid at some large $p_{T}$ where $\alpha_{s} \ln \left(Q^{2} / p_{T}^{2}\right) \ll 1$. By matching these two results in the overlap region of intermediate values of $p_{T}$, the accuracy of the resummed result is improved. The matching procedure accounts for terms not included in the resummation, that is, those that do not diverge as $1 / p_{T}^{2}$ as $p_{T} \rightarrow 0$. These terms can be extracted from the second order perturbative result by subtracting the $1 / p_{T}^{2}$ terms from it. The asymptotic $1 / p_{T}^{2}$ behavior of the perturbative result, as $p_{T} \rightarrow 0$, is obtained by expanding Equation 1.5 in powers of $\alpha_{s}$. This has been done to second order in $\alpha_{s}$. The result of matching the resummation result at low $p_{T}$ with the second order perturbative result at high $p_{T}$ can be expressed as:

$$
\frac{d \sigma}{d p_{T}}(\text { matched })=\frac{d \sigma}{d p_{T}}(\text { resummed })+\left[\frac{d \sigma}{d p_{T}}(\text { perturbative })-\frac{d \sigma}{d p_{T}}(\text { asymptotic })\right] .
$$




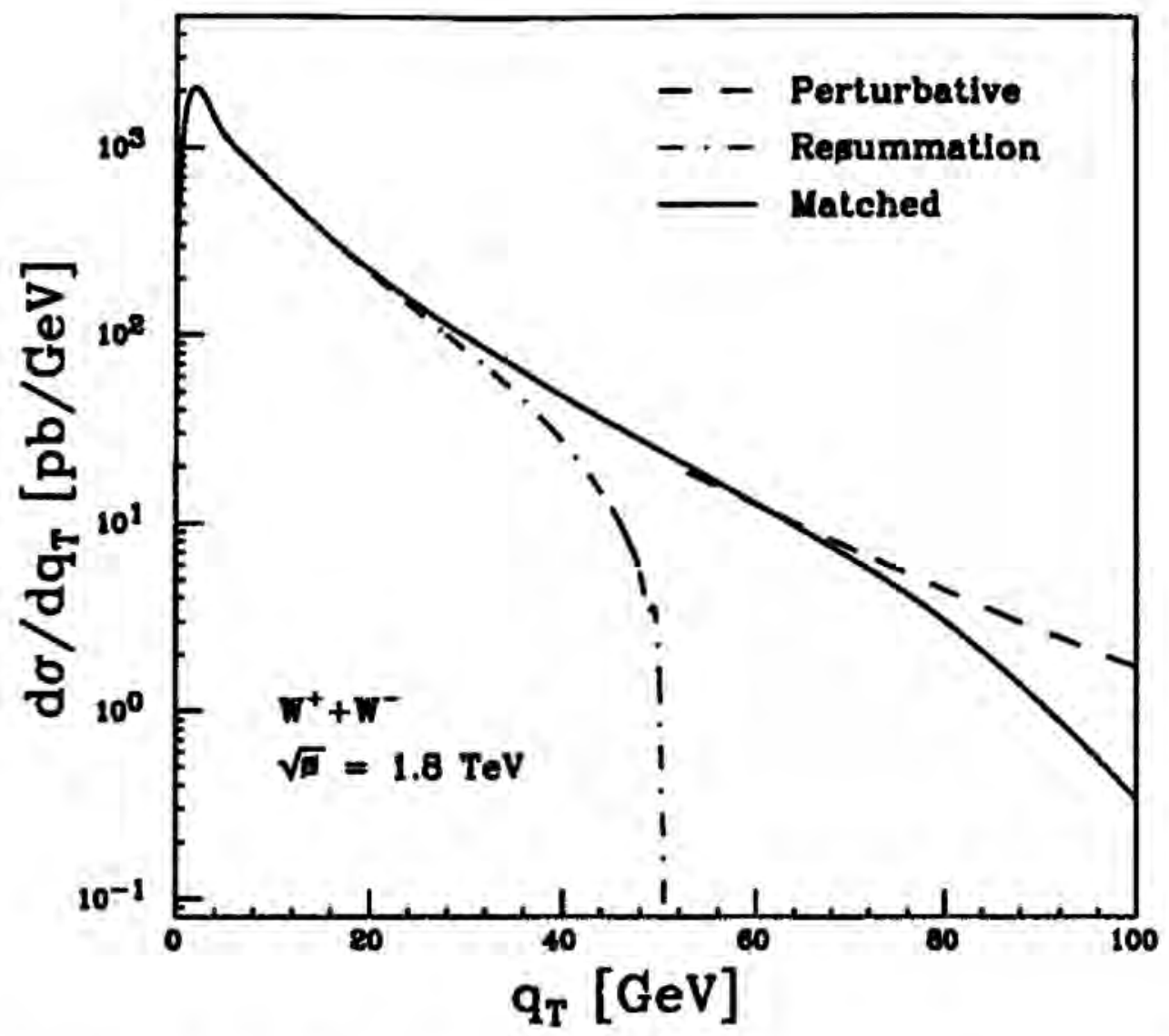

Figure 1,2: The $d \sigma / d p_{T}$ results calculated using the resummation, perturbative, and matching methods. The perturbative result is used for $p_{T}>50 \mathrm{GeV} / c$.

Note that at low $p_{T}$, the perturbative and the asymptotic pieces cancel, leaving the resummed piece; whereas at high $p_{T}$, the resummed and the asymptotic pieces cancel to second order in $\alpha_{s}$. Because this cancelation is not complete, the matched result becomes inaccurate at some large value of $p_{T}$, and the perturbative result is used. The process of matching is illustrated in Figure 1.2, where the results for $d \sigma / d p_{x}$ obtained from the different calculations are shown.

The next-to-leading order QCD prediction of $d \sigma / d p_{T}$, using the matching result for $p_{T}<50 \mathrm{GeV} / c$ and the perturbative result for $p_{T}>50 \mathrm{GeV} / c$, is plotted as a band in Figure 1.3; the width of the band indicates the uncertainty in the theoretical prediction. [8] The sources of the theoretical uncertainty are the following: 
1. The effects of non-perturbative physics. At small $p_{T}$, when $p_{T}<\Lambda_{Q C D}$, confinement effects sets in and $\alpha_{s}$ blows up. These non-perturbative effects are parametrized and are accounted for in the resummation procedure. Different choices of the parametrization leads to a $p_{T}$ dependent variation in the predicted $d \sigma / d p_{T}$. The variation is taken as a theoretical error. It is $\approx 40 \%$ at $p_{T}=1 \mathrm{GeV} / c$, and reduces to $\approx 2 \%$ at $p_{T}=10 \mathrm{GeV} / c$.

2. The choice of structure function and $\Lambda_{Q C D}$. The result using the HMRS(B) set [13] with $\Lambda_{Q C D}=190 \mathrm{MeV}$ is different from that using the HMRS(E) set with $\Lambda_{Q C D}=100$ $\mathrm{MeV}$ by 10 to $15 \%$.

3. The contributions from higher order processes. For the matching result, an estimate of the higher order contributions is simply taken as the difference between the matched and the perturbative results at $p_{T}=50 \mathrm{GeV} / c$. This procedure gives an uncertainty of $\approx 10 \%$ from neglecting higher order contributions.

4. The choice of renormalization and factorization scales. The uncertainty is estimated by choosing $Q^{2}=M_{Z}^{2}$ and $Q^{2}=p_{T}^{2}$. The difference in the perturbative result is $\approx 10 \%$. The matching result in not very sensitive to the choice of scale because the variation cancels out between the perturbative and the asymptotic results in Equation 1.6.

This detailed QCD calculation of the $W$ and $Z p_{T}$ distribution must be confronted with experimental tests. Previous studies of $W$ and $Z$ hadronic production have been performed at CERN in $\bar{p} p$ collisions at a center-of-mass energy of $0.63 \mathrm{TeV}$.[14] Results on the tota] production cross section as well as the $p_{T}$ distributions were in agreement with QCD predictions. The Fermilab Tevatron, with $\bar{p} p$ collisions at a center-of-mass energy of $1.8 \mathrm{TeV}$, provides an opportunity to test QCD in a new kinematic range. 


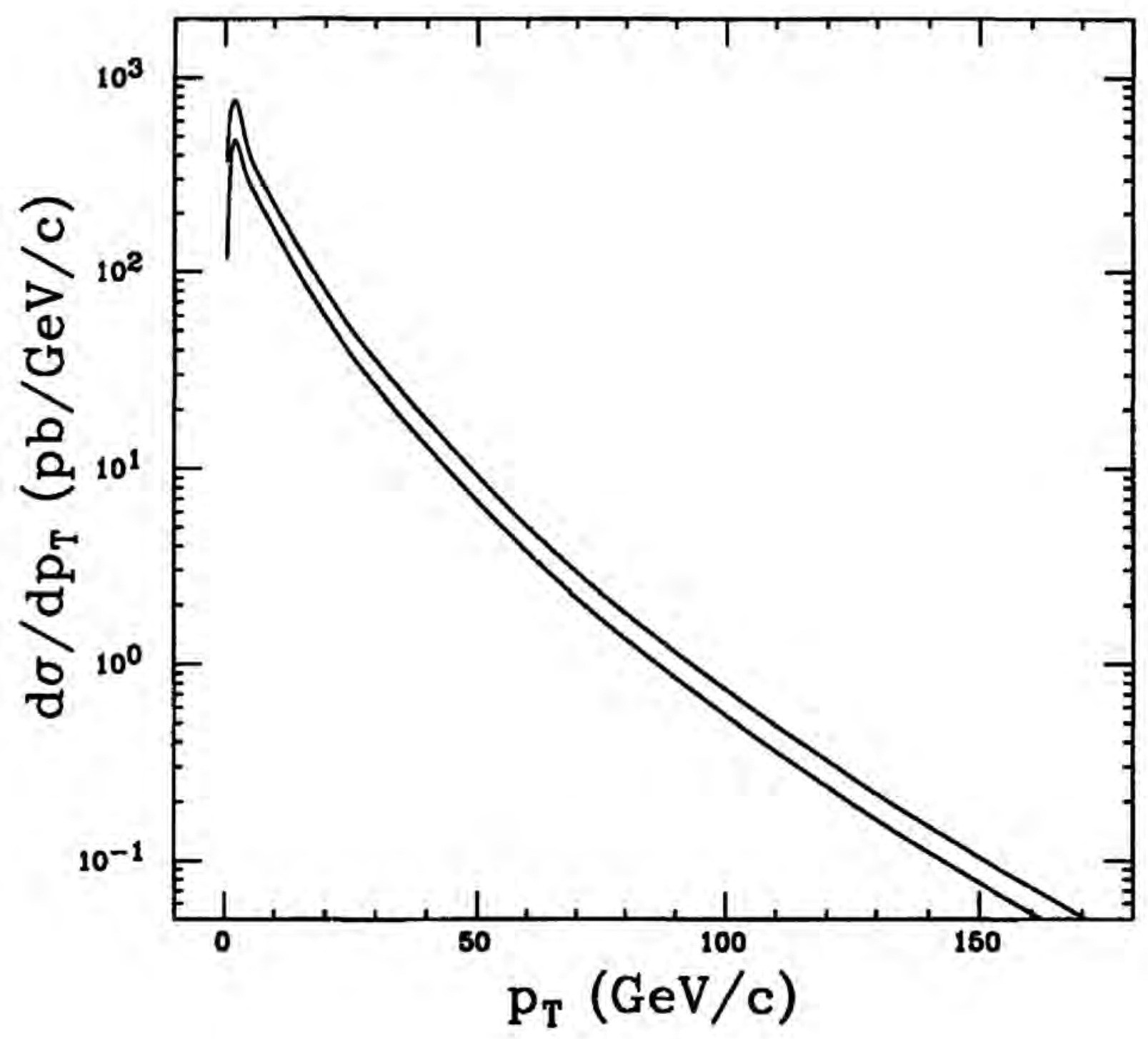

Figure 1.3: The next-to-leading order QCD prediction of $d \sigma / d p_{T}$ shown as a band; the width of the band indicates the theoretical uncertainty. 


\section{Chapter 2}

\section{Experimental Apparatus}

\subsection{The Fermilab Tevatron Accelerator}

The Fermi National Accelerator Laboratory, located in Batavia, Illinois, is host to the Tevatron, a proton-antiproton synchrotron accelerator.[15] The Tevatron complex consists of the proton source, the antiproton source, and the tunnel $2-\mathrm{km}$ in diameter housing the magnets and beam pipes of the Main Ring and the Tevatron ring. The general layout is shown in Figure 2.1, along with details of the antiproton source. The steps leading to $\bar{p} p$ collisions are outlined in the flow chart in Figure 2.2. Typical performance parameters of the Tevatron are summarized in Table 2.1.

During the 1988-89 data collection period, the Tevatron delivered a peak luminosity of $2 \times 10^{30} \mathrm{~cm}^{-2} \mathrm{sec}^{-1}$ at $\sqrt{s}=1.8 \mathrm{TeV}$, and a total of $10 \mathrm{pb}^{-1}$. The data written to tape corresponds to approximately half of the total delivered luminosity.

Table 2.1: Typical beam parameters of the Tevatron.

\begin{tabular}{c|c}
\hline Parameter Description & Parameter Value \\
\hline Beam Energy & $900 \mathrm{GeV}$ \\
Number of Bunches & 6 \\
Number of Protons/Bunch & $7 \times 10^{10}$ \\
Number of anti-Protons/Bunch & $2.5 \times 10^{10}$ \\
Luminosity & $1.8 \times 10^{30} \mathrm{~cm}^{-2} \mathrm{sec}^{-1}$ \\
Luminosity Lifetime & 20 hours \\
\hline
\end{tabular}


a)

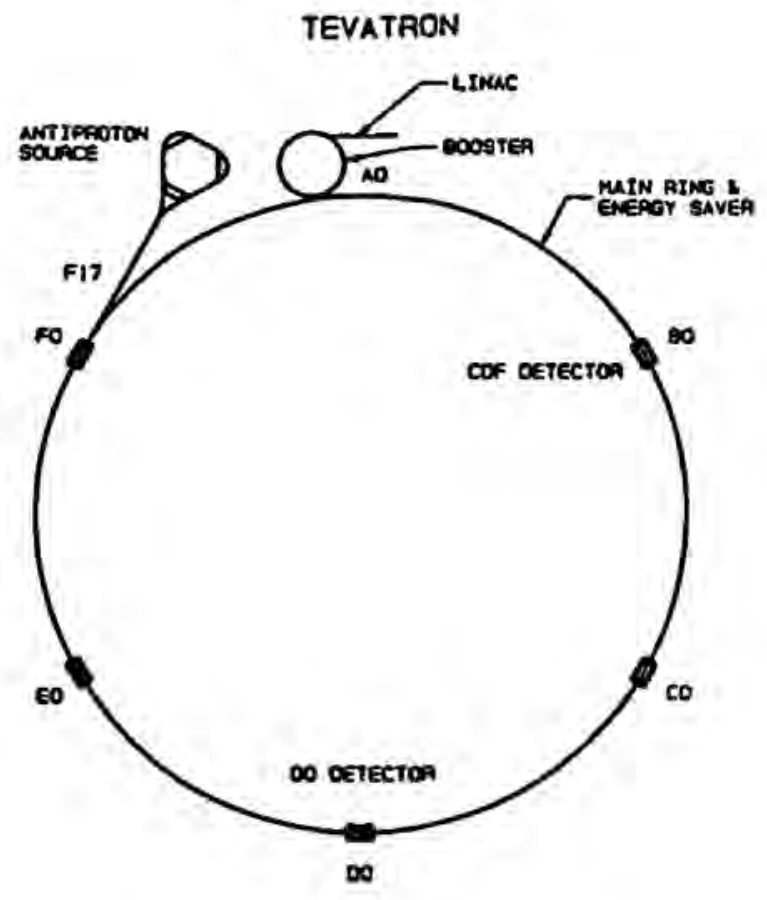

b)

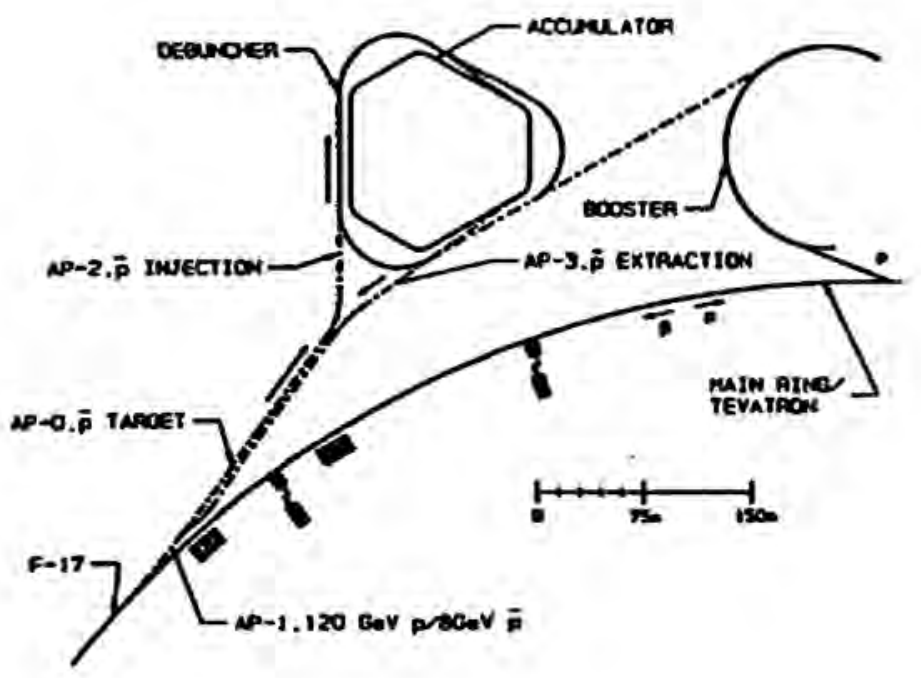

Figure 2.1: General lay-out of the Fermilab a)Tevatron accelerator, and the b)antiproton source. 


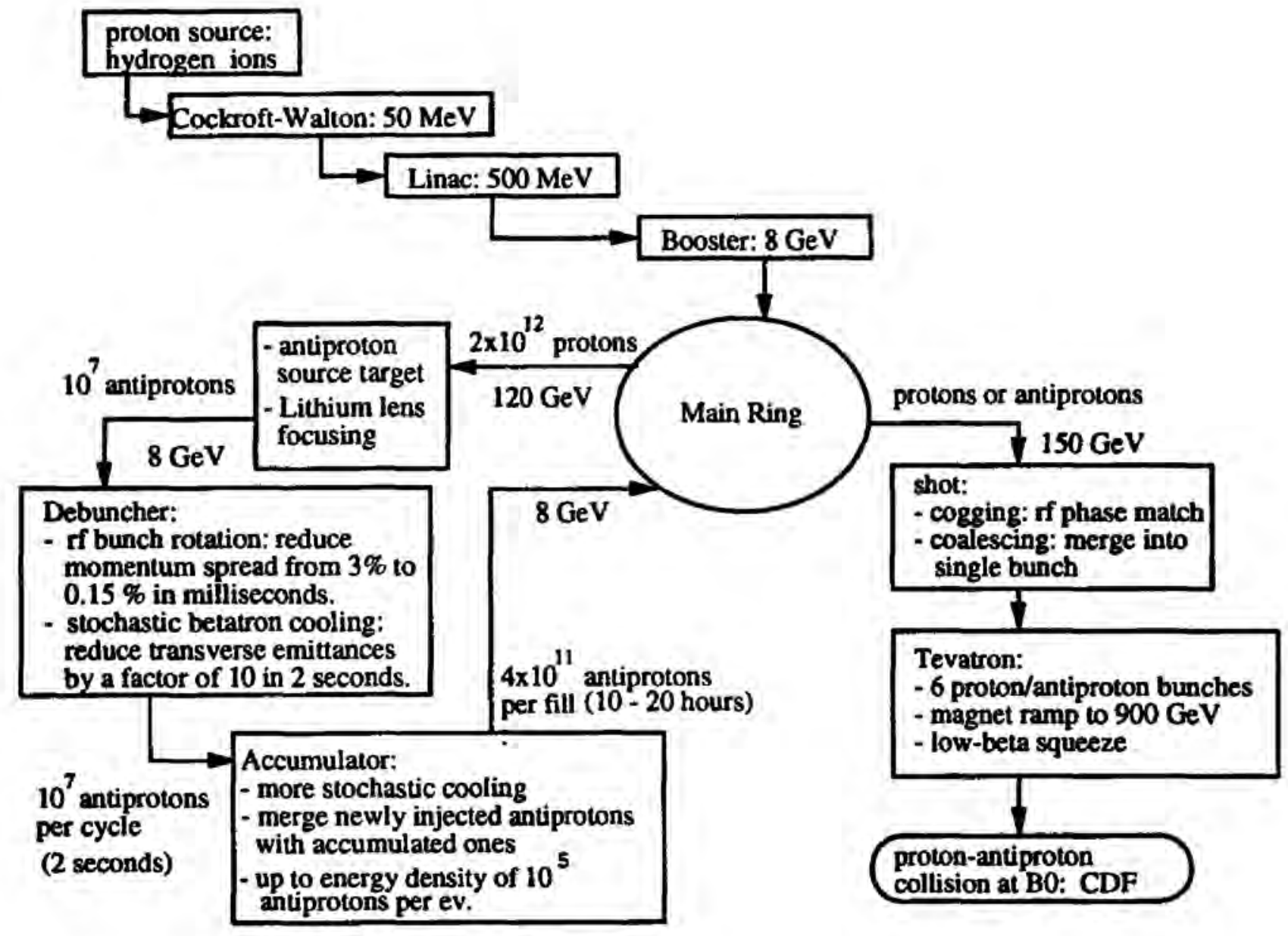

Figure 2.2: Flow chart illustrating the Tevatron operation cycle. 


\subsection{The Collider Detector at Fermilab (CDF)}

In contrast to the few counters needed in Rutherford's experiment, the complexity of high energy hadron-hadron scattering processes in the $\mathrm{TeV}$ range places stringent requirements on a detector's capability. To explore the wide variety of physics phenomena at the Tevatron, the CDF [17] detector was designed to be able to measure the energy and momentum of leptons and quark/gluon jets over a large solid angle. It is also expected to be able to identify these particles with good efficiency. This goal is achieved by a combination of charged particle tracking detectors, sampling calorimeters, and muon detectors surrounding the interaction region. Information from these detectors must be available at the trigger level to select high momentum transfer scattering events among the bulk of interactions resulting from "soft" processes.

At a hadron collider, the constituent scattering process takes place in a system with net longitudinal motion with respect to the laboratory because the colliding partons do not have the same momentum. It is therefore desirable to use quantities that are insensitive to a longitudinal Lorentz boost when describing these scatterings. One such quantity is the transverse energy, $E_{T}=E \sin \theta$, where $\theta$ is the polar angle with respect to the beam axis. For a massless particle, $E_{T}$ is the same as $p_{T}$ which is invariant. Also, energy is more accessible via calorimeter measurement than momentum is via tracking. The other useful quantity

is rapidity, $y=\frac{1}{2} \ln \left(\frac{E+p_{z}}{E-p_{z}}\right)$, where $p_{z}$ is the component of the particle momentum along the proton beam direction ( $z$-axis). It is known that in "soft" processes, which constitute most of the hadronic interactions, particle production at a given $E_{T}$ is uniform in azimuth $(\phi)$ and rapidity. Rapidity also has the property of being additive under a longitudinal Lorentz boost. To explore these characteristics of hadron collisions, CDF is constructed with a readout segmentation uniform in $\phi$ and pseudo-rapidity, $\eta=-\ln [\tan (\theta / 2)]$. For a massless particle, pseudo-rapidity is the same as rapidity. Some details of the CDF detector are shown in Figures 2.3 and 2.4. 


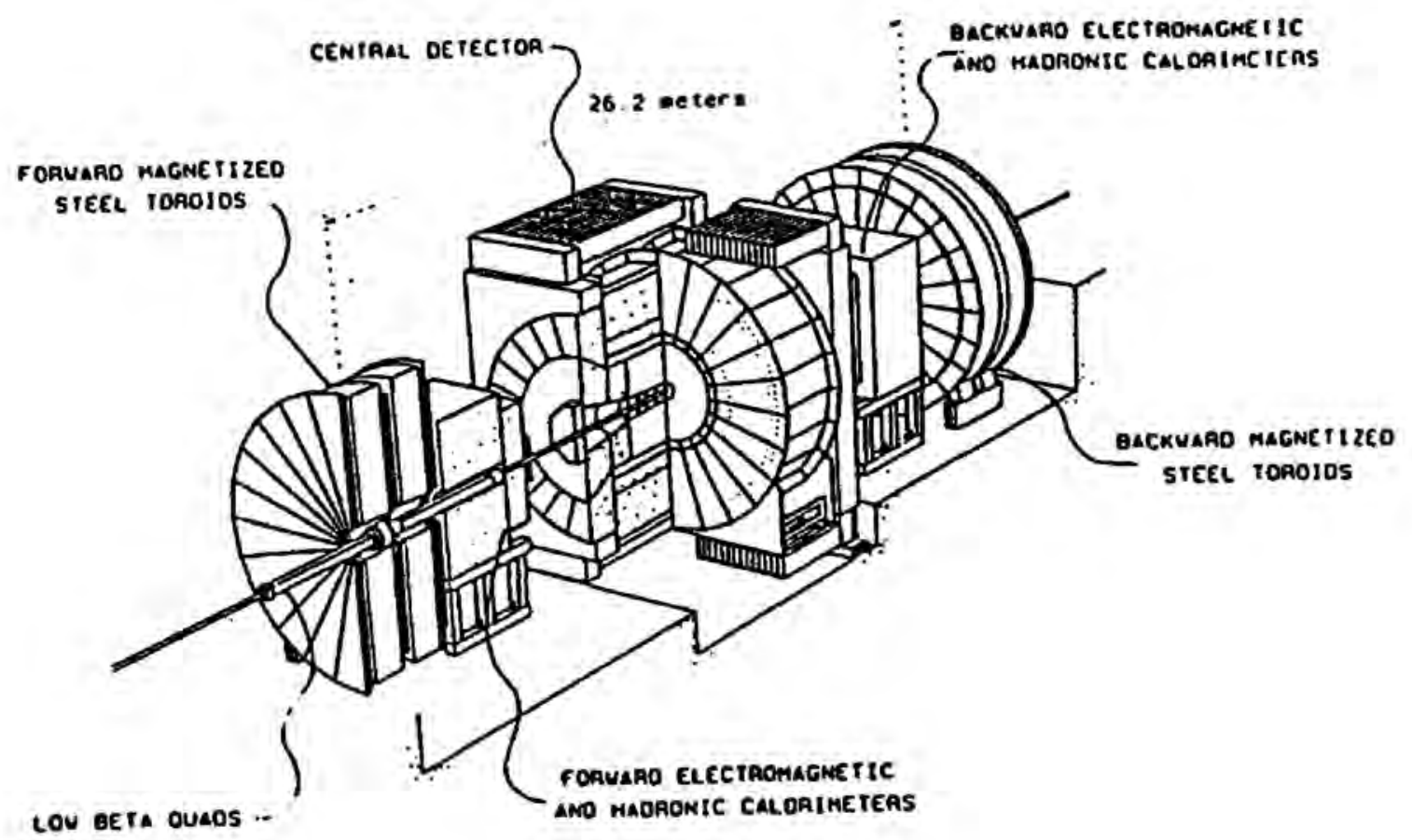

Figure 2.3: The CDF detector: a perspective view of the various components showing nearly complete $4 \pi$ coverage. 


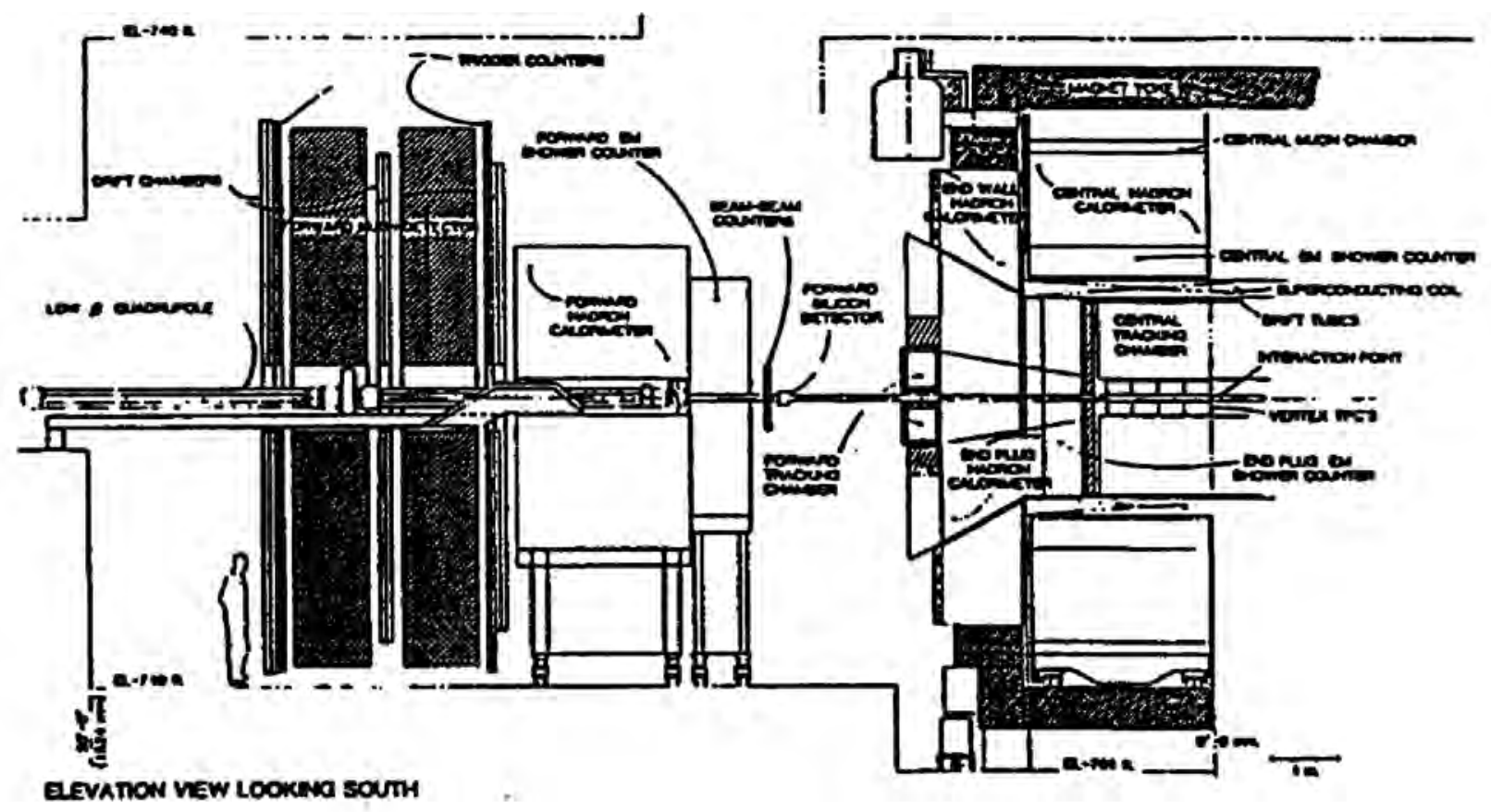

Figure 2.4: The CDF detector: a cut-away view showing symmetry about the beam line; the other half is symmetric about the midplane. 


\subsubsection{Tracking Devices}

The Vertex Time Projection Chamber (VTPC) system, shown in Figure 2.4 surrounding the beam pipe, is designed to provide event vertex determination, identify multiple interactions in the same beam crossing, and provide tracking information over a wide range of polar angles. It is constructed with low-mass material to reduce photon conversions in its active volume. It consists of 8 modules, mounted end-to-end along the beam direction. In each module, electrons drift away from a central high voltage grid toward endcaps divided into octants. The arrival times at the sense wires provide $r-z$ information. Charge distribution in the pads and the relative rotation in $\phi$ of alternate modules provide some $\phi$ information. For central tracking, only the $r-z$ information is used to determine the vertex position along the $z$ direction. The primary $z$-vertex resolution is $\approx 1 \mathrm{~mm}$.

Surrounding the VTPC is the Central Tracking Chamber (CTC), shown in Figure 2.5. The primary function of the CTC is to provide momentum measurement on charged particles at the trigger level as well as in offline data analysis. It is designed to handle high multiplicity ( $\approx 4$ charged particles per unit rapidity typical of "soft" processes) and a high interaction rate $(\approx 50 \mathrm{kHz})$, and operate inside a 1.4 Tesla magnetic field provided by a superconducting coil.

The CTC is a $3.2 \mathrm{~m}$ long cylindrical drift chamber, with an inner radius of $0.28 \mathrm{~m}$, an outer radius of $1.38 \mathrm{~m}$, and 84 layers of sense wires arranged into 9 superlayers. Five axial superlayers, each containing 12 sense wire layers parallel to the beam axis, provide $r-\phi$ tracking. Interleaved are four stereo superlayers, each containing 6 sense wires tilted at $\pm 3^{\circ}$ with respect to the beam axis, that provide $r-z$ tracking. Each superlayer is divided into cells defined by two planes of field wires with maximum drift distance less than 40 $\mathrm{mm}$ (drift time of less than $800 \mathrm{~ns}$ ). Each cell is tilted at $45^{\circ}$ to the radial direction, as shown in Figure 2.5. The tilt compensates for the Lorentz angle, so that the drift trajectories of electrons from the ionization are roughly azimuthal. Tilting the cells also helps to resolve closely spaced tracks because the cells now overlap, simplifies resolving the 
a)

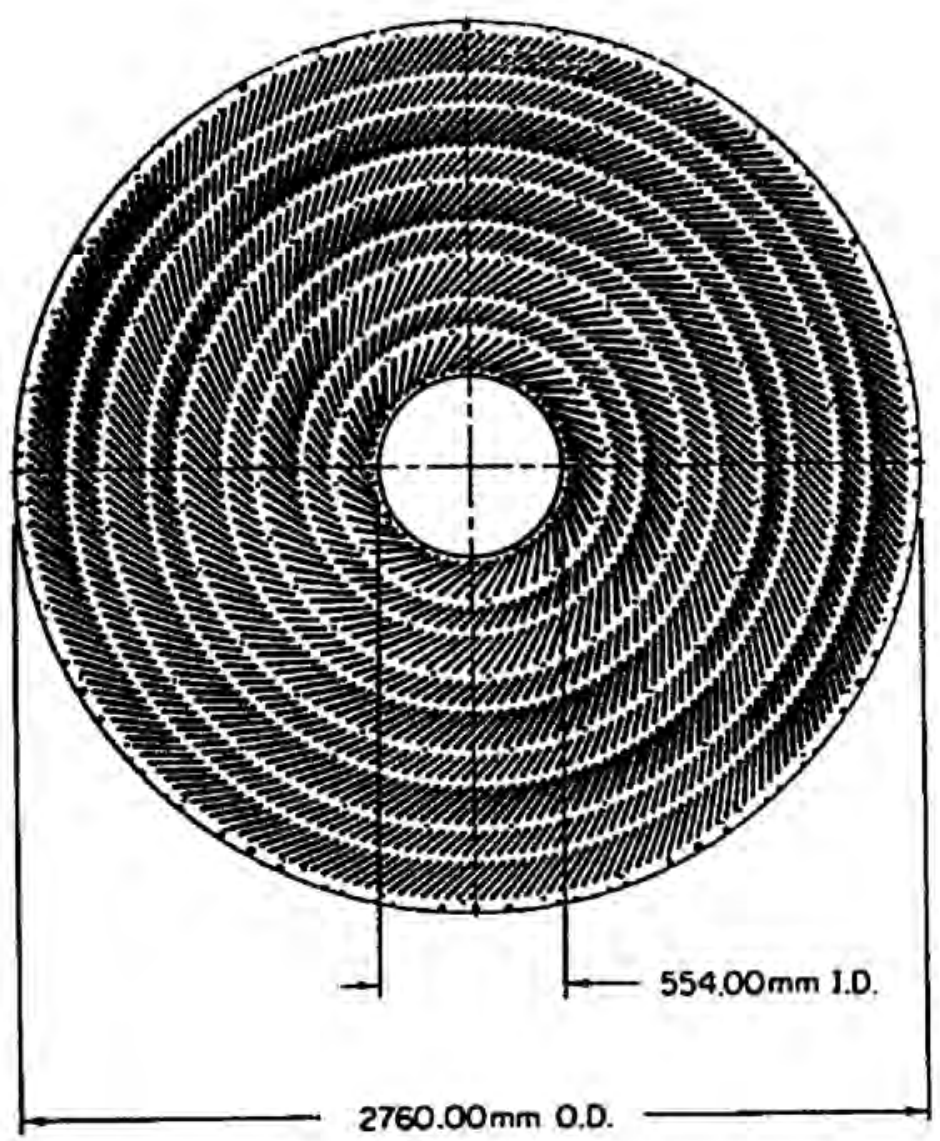

b)

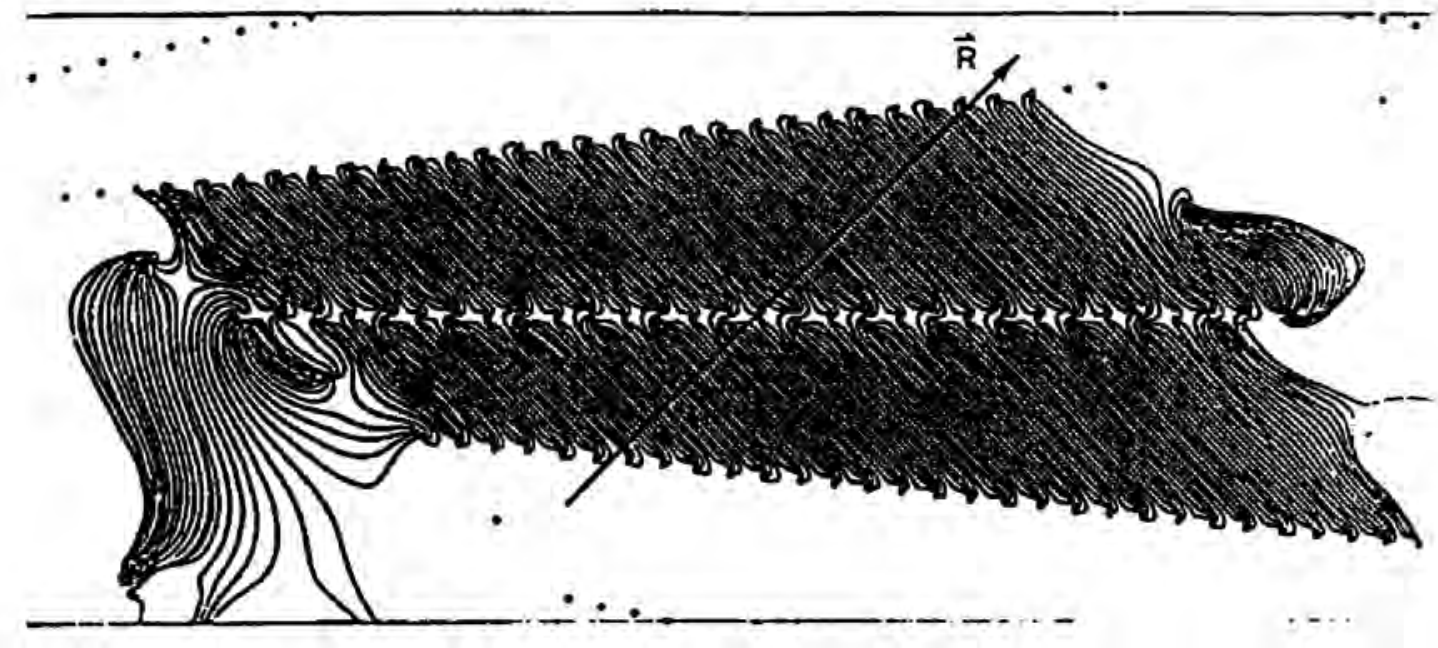

Figure 2.5: The CTC: a) End view showing 9 superlayers, b) Trajectories in a drift cell; the arrow indicates the radial direction. 
left-right ambiguities because the ghost track would be pointed away from the beam axis by a large angle, and allows radial tracks to sample the whole range of drift distances in the cell. For tracks passing all 9 superlayers, in the region $|\eta|<1.0$, the CTC momentum resolution is $\delta p_{T} / p_{T}=0.17 \% p_{T}(\mathrm{GeV} / \mathrm{c})$. By constraining the tracks to come from the beam spot, determined for each Tevatron store by studying the the impact parameter distribution as a function of $\phi$, the momentum resolution is improved to $\delta p_{T} / p_{T}=0,11 \% p_{T}(\mathrm{GeV} / \mathrm{c})$. Details of the track recognition and fitting procedures are discussed in Section 3.2.1.

\subsubsection{Calorimeters}

Calorimeters are used to measure the energy of charged and neutral particles, and provide shower profile and position information essential to electron identification. Calorimeters are organized into a projective tower geometry, i.e., each tower points back to the interaction region, with electromagnetic compartments followed by hadronic compartments. Calorimeters covering the central region, $|\eta|<1.1$, use scintillator as the active detector medium. Calorimeters covering the plug and forward regions, $1.1<|\eta|<2.4$ and $2.4<|\eta|<4.2$, respectively, use gas proportional tubes. In the central and plug electromagnetic calorimeters, proportional chambers are imbedded near shower maximum to measure shower position and shape. Calorimeter segmentation and coverage in $\eta \cdot \phi$ are shown in Figure 2.6.

\section{The Central Electromagnetic Calorimeter (CEM)}

The central EM calorimeter, surrounding the central tracking chamber, consists of 4 " $\mathrm{C}^{\text {" }}$. shaped arches. Each arch has twelve $15^{\circ}$ azimuthal wedges. Each wedge has 10 towers. Each tower, covering $\approx 0.11$ in $\eta$ and $15^{\circ}$ in $\phi$, is constructed with 30 layers of $5 \mathrm{~mm}$ thick

polystyrene scintillator interleaved with $\frac{1}{8}$ inch lead. It has a single sampling depth, with a proportional chamber inserted near shower maximum This chamber consists of 64 wires along the $z$-direction providing the $x$-view, and 128 strips along the $x$-direction providing the $z$-view of the shower position and profile. The position resolution is $2 \mathrm{~mm}$ for 50 $\mathrm{GeV} / \mathrm{c}$ electrons. The thickness of the $\mathrm{EM}$ compartment is $\approx 18$ radiation lengths and $\approx 1$ absorption length. As $\theta$ varies, constant radiation length is obtained by substituting 


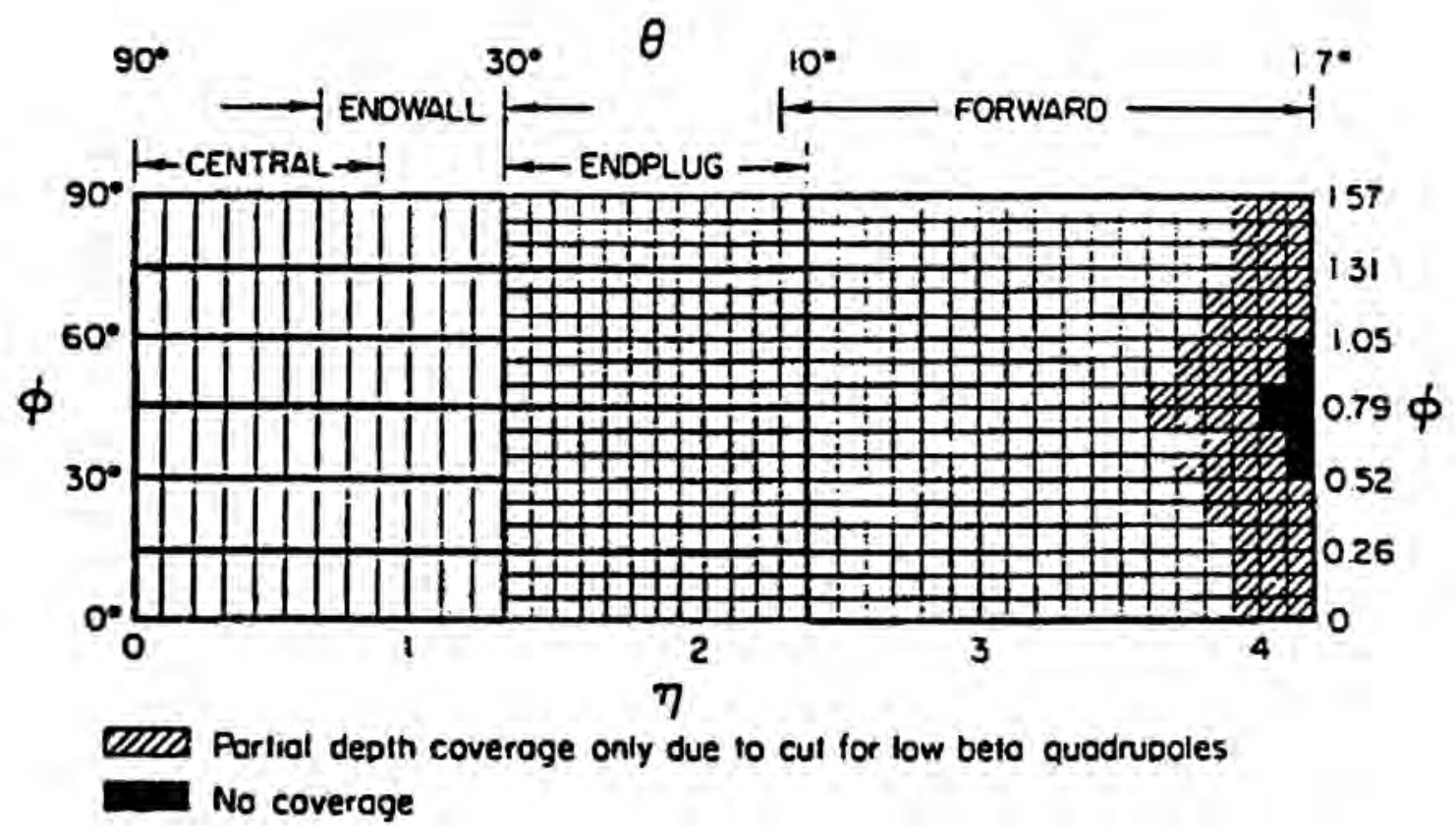

Figure 2.6: The calorimeter $\eta$ - $\phi$ segmentation.

acrylic for some of the lead. Light is collected on the two azimuthal sides of the tower with wave-shifters attached to phototubes mounted inside magnetic shields. The light gathering layout of a central wedge is shown in Figure 2.7.

Each wedge was calibrated at the test beam, using the response to $50 \mathrm{GeV} / \mathrm{c}$ electrons at the tower center. The calibration is maintained by the response to ${ }^{137} \mathrm{Cs}$ sources that inject a signal into the scintillator, and monitored by a system of xenon flash tubes that inject light into the waveshifter and light emitting diodes (LEDs) that inject light into the phototubes. The calibration system monitors any radiation damage, ageing, or effects due to the magnetic field. Variation of energy response over a tower due to light attenuation is less than $1 \%$ from studies at the test beam. Tower-to-tower calibration is obtained from inclusive electron data. The absolute energy scale is set by studying the $E / p$ distribution of $W$ electrons. The CEM energy resolution is $\left(\sigma_{E} / E\right)^{2}=\left(0.135 / \sqrt{E_{T}(\mathrm{GeV})}\right)^{2}+(0.02)^{2}$. 


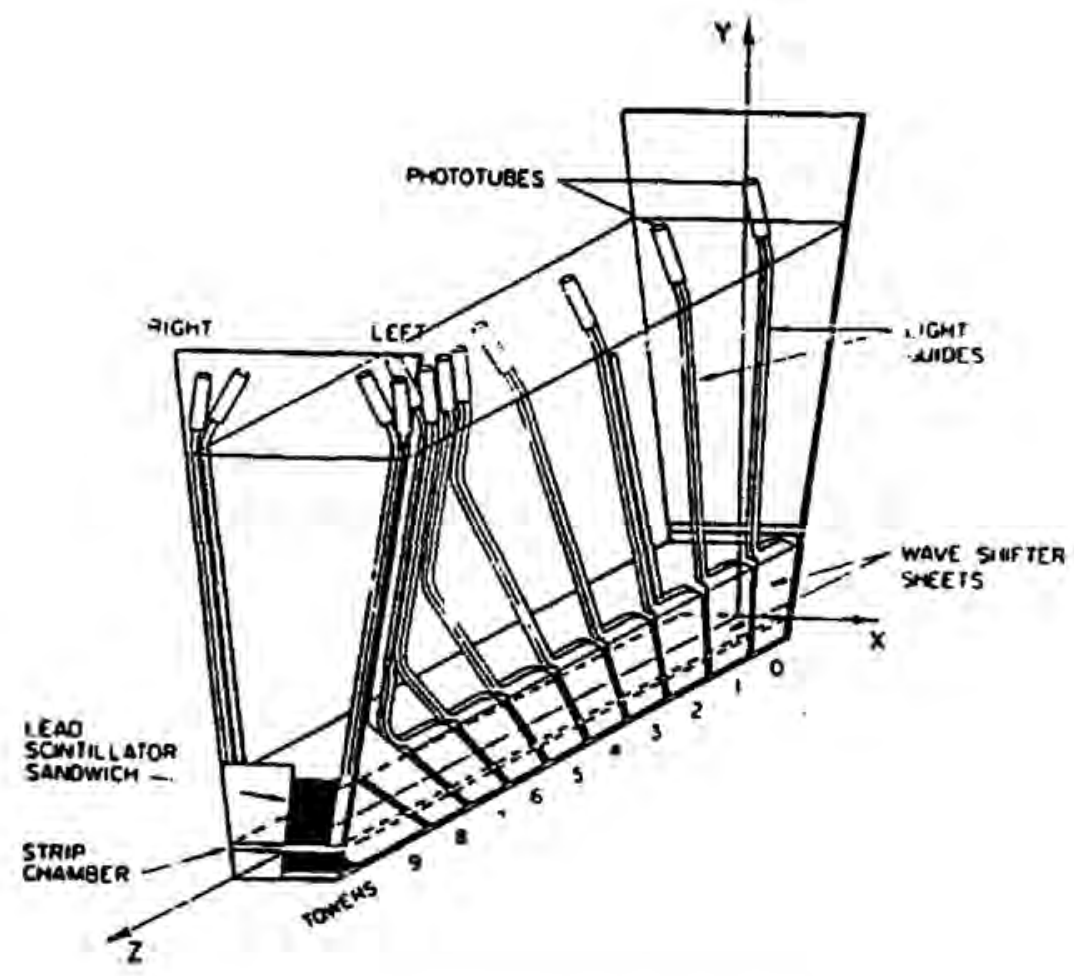

Figure 2.7: The light gathering layout of one CEM module.

The CEM energy scale calibration is discussed in more detail in Section 3.2.2.

The Central and Endwall Hadron Calorimeters (CHA and WHA)

Immediately surrounding the CEM are the hadron calorimeters with matching $\boldsymbol{\eta}-\phi$ segmentation. The central hadron calorimeter (CHA), covering $|\eta|<0.8$, is constructed with 32 layers of $1 \mathrm{~cm}$ thick scintillator interleaved with $2.5 \mathrm{~cm}$ thick steel. The endwall (WHA) calorimeter, covering $0.8<|\eta|<1.1$, is constructed with 15 layers of $1 \mathrm{~cm}$ thick scintillator interleaved with $5 \mathrm{~cm}$ steel. Each hadronic tower is $\approx 4.7$ absorption length thick.

Each CHA module was calibrated at the test beam, using the response to pions in the energy range 10 to $150 \mathrm{GeV} / \mathrm{c}$ at the tower center. The calibration is maintained by the response to ${ }^{137} C s$ sources. Module-to-module calibration is tracked to within $4 \%$ by the sources. Two WHA modules were calibrated at the test beam. The rest of the modules were calibrated by cross-referencing the responses to sources. There is also a monitoring 
system that distributes laser light pulses to the phototubes and checks the linearity and the ADC range calibration. In addition, ageing effects are monitored by ${ }^{137} \mathrm{Cs}$ sources. Below $50 \mathrm{GeV}$, energy resolution is affected by the sampling thickness; for higher energy, leakage effects are dominant. At the tower $\theta$ edges, response is lower by about $10 \%$ due to lower wavelength shifter light output. At tower $\phi$ interfaces, there are hot-spots due to Cherenkov radiation of particles showering in lightguides. This energy deposition can be recognized as anomalous left-right phototube output ratio and are removed in offline analysis. Further analysis of test beam data reveals nonlinearity in response at low energy and must be taken into account in jet energy measurements. (See Section 5.4.)

\section{The Gas Calorimeters}

All four gas calorimeters have similar construction, readout, monitoring and calibration schemes. Each uses gas sampling calorimetry, with lead or steel interleaved with layers of proportional tubes. The proportional tubes and cathode pads are read out by sample-andhold circuits. Cathode pads are etched out of copper plating with uniform segmentation $0.1 \times 5^{\circ}$ in $\eta-\phi$. The gas used is 50-50 argon-ethane with small admixture of isopropyl/ethanol alcohol at low temperature to prevent glow discharge. The response of gas calorimeters is sensitive to change in operational conditions such as the high voltage, the gas temperature, the gas pressure, and the gas composition. The gain of the gas is monitored by measuring the peak of the $5.9 \mathrm{keV} \mathrm{X}$-rays from ${ }^{55} \mathrm{Fe}$ sources using proportional tubes similar to those in the calorimeter.

The responses of the gas calorimeters and the gas gain monitoring tubes were studied at the test beam. During the 1988.89 run, the responses of tubes mounted on each detector and sampling the same gas as the detector, tracked each other to within $3 \%$. The average of the responses of these tubes, for each detector on each side of the collision hall, was used as the gas gain calibration. These calibrations were downloaded to the trigger before each run to compensate for calorimeter response variations. Gas composition was checked before usage, and maintained to within $1 \%$ by re-mixing if necessary. 


\section{The End Plug Electromagnetic Calorimeter (PEM)}

Covering the region $1.1<|\eta|<2.4$, or $10^{\circ}<|\theta|<36^{\circ}$, the plug electromagnetic calorimeter is constructed with a conical hole of $10^{\circ}$ and a cylindrical outer shape. Each side consists of 4 quadrants, $90^{\circ}$ each in $\phi$. Each quadrant has 34 layers of proportional tube arrays interleaved with $2.7 \mathrm{~mm}$ thick lead. Pads on each layer are etched out of copper plating clad on G-10 panels with segmentation $0.1 \times 5^{\circ}$ in $\eta-\phi$. Longitudinally, pads are ganged together to form a projective tower in three depth segments. Near shower maximum, from layer 6 to layer 15 , for $1.2<|\eta|<1.84,32$ arc-shaped $\theta$-strips with $\Delta \eta=0.02$ and $\Delta \phi=30^{\circ}$, and 30 radial $\phi$-strips with $\Delta \phi=1^{\circ}$ are imbedded to measure shower profile and position. The angular resolution is $0.04^{\circ}$ for $\theta$-strips and $0.1^{\circ}$ for $\phi$-strips. In the $\eta$ region not covered by strip chambers, the physical size of the pads is small enough for good shower shape and position measurement.

At the test beam, all 2304 towers were scanned by $100 \mathrm{GeV}$ electrons at the tower center. The energy resolution was determined to be $\sigma_{E} / E=28 \% / \sqrt{E(\mathrm{GeV})} \pm 2 \%$. The longitudinal shower profile at different incident electron energies and angles indicate $\approx 4 \%$ leakage at $200 \mathrm{GeV}$. The response variation in $\phi$ is $\approx 10 \%$ peak-to-peak when averaged over $\eta$. The variation in $\eta$ is $\approx 20 \%$ peak-to-peak when averaged over $\phi$. A correction map is obtained and is used in offline analysis to correct the electron energy measurement. Quadrant-to-quadrant response variation is obtained from $W$ and $Z$ data. See Section 3.2.2 for more details on PEM electron energy corrections.

\section{The End Plug Hadronic Calorimeter (PHA)}

Immediately behind the PEM is the plug hadron calorimeter. Each side consists of 12 sectors, $30^{\circ}$ each in $\phi$. Each sector consists of 20 sampling layers of proportional tubes interleaved with $5 \mathrm{~cm}$ steel plates for a total of 6.5 absorption lengths. Pads from each layer are ganged together to form a projective tower in one depth segment with $\eta$ - $\phi$ segmentation identical with the PEM. Wires for each layer of the sector are also read out. The energy scale was set at the test beam with pions at the central tower of a sector. The Energy 
resolution was measured to be $\sigma_{E} / E=86 \% / \sqrt{E(\mathrm{GeV})}+4 \%$.[18]

The Forward Electromagnetic Calorimeter (FEM)

Extending the coverage down to $\approx 2^{\circ}$ in $\theta$ on both sides, or $2.2<|\eta|<4.2$, the forward electromagnetic calorimeter is located $\approx 6.5 \mathrm{~m}$ from the nominal interaction point. Each side consists of 4 quadrants, $90^{\circ}$ each in $\phi$. Each quadrant consists of 30 sampling layers of proportional tubes interleaved with $48 \mathrm{~mm}$ thick lead plates. Each layer is self-contained and can be replaced when necessary. Longitudinally, pads are ganged together to form a projective tower in two depth segments. Anode wires strung vertically and ganged together in five sectors are read out at each layer.

Performance of the FEM was studied with test beam electrons 20 to $200 \mathrm{GeV}$ in energy. The energy response is linear up to $100 \mathrm{GeV}$, with a $10 \%$ non-linearity at $200 \mathrm{GeV}$. The energy resolution was determined to be $\sigma_{E} / E=25 \% / \sqrt{E(\mathrm{GeV})} \pm 0.5 \%$. The quadrant-toquadrant response variation is obtained from $\bar{p} p$ data, using the energy flow of low energy neutrons. Correction to the overall energy scale is obtained from $Z$ events. These are discussed in more detail in Section 3.2.2.

\section{The Forward Hadronic Calorimeter (FHA)}

Behind the FEM is the FHA. Each quadrant of the FHA consists of 27 layers of proportional tubes interleaved with $5 \mathrm{~cm}$ thick steel plates. Cathode pads are ganged together to form a projective tower in one depth segment. The energy response was studied at the test beam with pions. The energy resolution is $\sigma_{E} / E=93 \% / \sqrt{E(\mathrm{GeV})} \pm 1.5 \%$.[18] The relative response of each quadrant is obtained from $\bar{p} p$ data using di-jet balancing and neutron energy flow.

\subsubsection{Muon Detector}

The Central Muon detector consists of drift chambers located behind $\approx 6$ hadronic absorption lengths of the central calorimeter at $347 \mathrm{~cm}$ from beam axis and cover the region $0.03<|\eta|<0.63$. (In $\theta$, the ranges are $88.5^{\circ}$ to $55.9^{\circ}$ and $91.5^{\circ}$ to $124.1^{\circ}$.) The drift chambers are arranged in wedges $12.6^{\circ}$ in $\phi$ that fit onto top of the CHA wedges. Each 
muon wedge is further divided into 3 modules $4.2^{\circ}$ each in $\phi$. Each module consists of 4 layers of 4 rectangular drift cells. Two pairs of sense wires from alternate layers are radially aligned, with each pair offset by $2 \mathrm{~mm}$ from the other. Drift arrival times at one pair of wires determine the track angle; the other pair is used to resolve the $\phi$ ambiguity. This setup allows momentum measurement for a level-1 trigger. The location of the drift chamber wedge and arrangement of the 4 layers are shown in Figure 2.8. The wires in alternate cells of the same layer are tied together at the $90^{\circ} \theta$-end with charge and arrival time read out separately at the $56^{\circ} \theta$-end. The track position along the wire is determined from charge division. To account for variation in charge collection, each sense wire is calibrated with ${ }^{55}$ Fe sources.

Cosmic ray test results indicate a rms resolution of $1.2 \mathrm{~mm}$ along the wire and $0.25 \mathrm{~mm}$ in the $\phi$ direction. Studies with test beam pions indicate pion punch through of $10 \%$ at 15 $\mathrm{GeV}$ and $40 \%$ at $50 \mathrm{GeV}$. For $Z \rightarrow \mu \mu$ event selection, the second muon can be identified with good efficiency from a high $p_{T}$ track with the requirement that the energy deposition in the calorimeter be consistent with that from a minimum ionizing particle. Details of muon reconstruction and selection for $Z \rightarrow \mu \mu$ events are discussed in Sections 3.2 .3 and 4.2 , respectively.

\subsubsection{The Data Acquisition System}

The CDF online data acquisition system consists of a front-end system, a multi-level trigger system for selecting interesting events, an online micro-computer system for reformatting a complete event into CDF offline data structure, and an array of parallel processors, also known as the level 3 trigger, for selecting events to be sent to host VAX computers and written to tape.

In the CDF, the interaction rate is $\approx 50 \mathrm{kHz}$ at an instantaneous luminosity of $10^{30}$ $\mathrm{cm}^{-2} \mathrm{sec}^{-1}$. The bulk of these interactions result from "soft" processes. We are mainly interested in the physics of hard scattering processes with high momentum transfer. The data output rate is constrained by the tape writing limit, which is $\approx 1 \mathrm{~Hz}$. Thus, the trigger 
a)

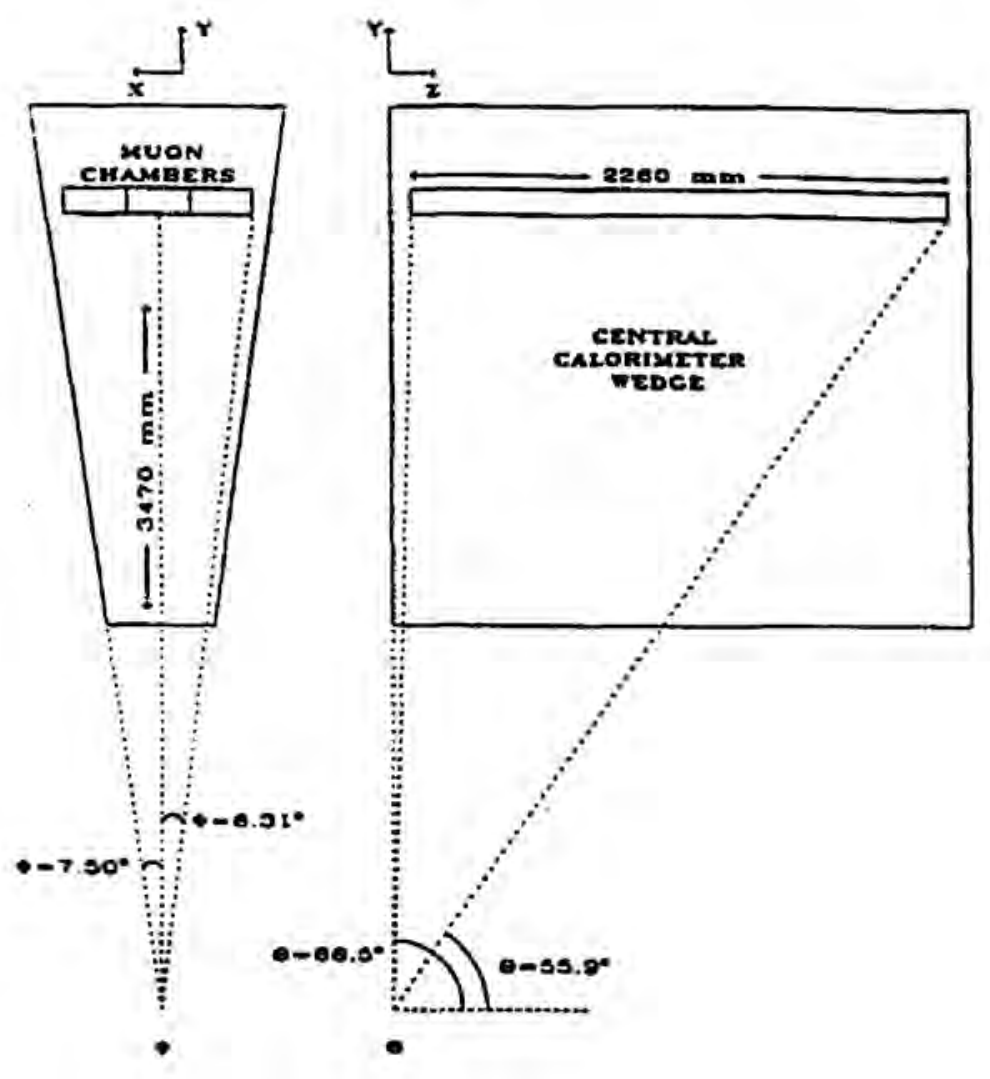

b)

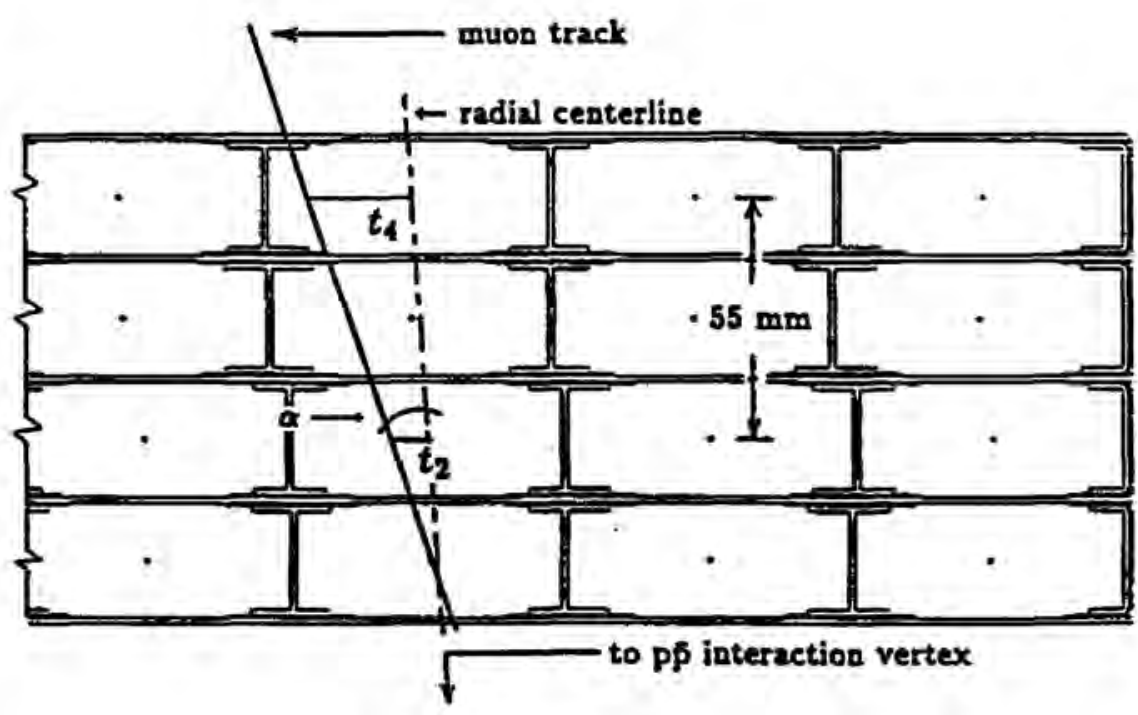

Figure 2.8: The Central Muon drift chamber system. a) The location of the muon detector within the central calorimeter. b) The cross sectional view of a single muon drift chamber, showing the drift times and the track angle. 
must be able to select interesting events to write to tape. It is a 4-level trigger:

- Level 0 . It simply requires the time-of-flight counters on both side of the interaction region to have hits in coincidence.

- Level 1. It makes a decision based on the total transverse energy deposition in the calorimeter and the presence of stiff tracks and muon drift chamber hits. The hardware track finder uses arrival times of CTC hits to indicate the presence of a track. The level-1 muon trigger forms track segments from hits in muon chambers, and makes a cut in $r-\phi$ angle equivalent to a $p_{T}$ threshold cut. A Level 1 reject resets the front-end electronics for the next event. The Level 1 trigger rate is a few $\mathrm{kHz}$.

- Level 2. It is based on event topological features such as the presence of energy clusters, high $p_{T}$ CTC tracks, and muon drift chamber hits. The cluster finder takes a seed tower and adds up nearest neighbor towers above threshold. The Central Fast Track (CFT) [19] finder compares CTC hits to pre-determined hit patterns for two-dimensional tracks with a given $p_{x}$ threshold. The $\phi$-information of the CFT track can be matched to an electromagnetic (EM) cluster for the electron trigger, or a muon drift chamber track segment for the muon trigger. The information collected at level two is passed on to programmable FASTBUS modules to generate trigger accept/reject signals. A level 2 accept initiates readout of the front end scanners. The Level 2 trigger rate is typically $5 \mathrm{~Hz}$.

- Level 3. It runs offline algorithms to reconstruct calorimeter energy clusters and a fast track finding algorithm for better track $p_{T}$ and $\phi$ measurement. The Level 3 trigger was implemented during the run. The electron analysis is based on the Level 2 trigger, while the muon analysis uses the Level 3 trigger information. The Level 3 trigger rate is typically $\mathrm{i} \mathbf{H z}$.

The event processing flow is shown in Figure 2.9. 


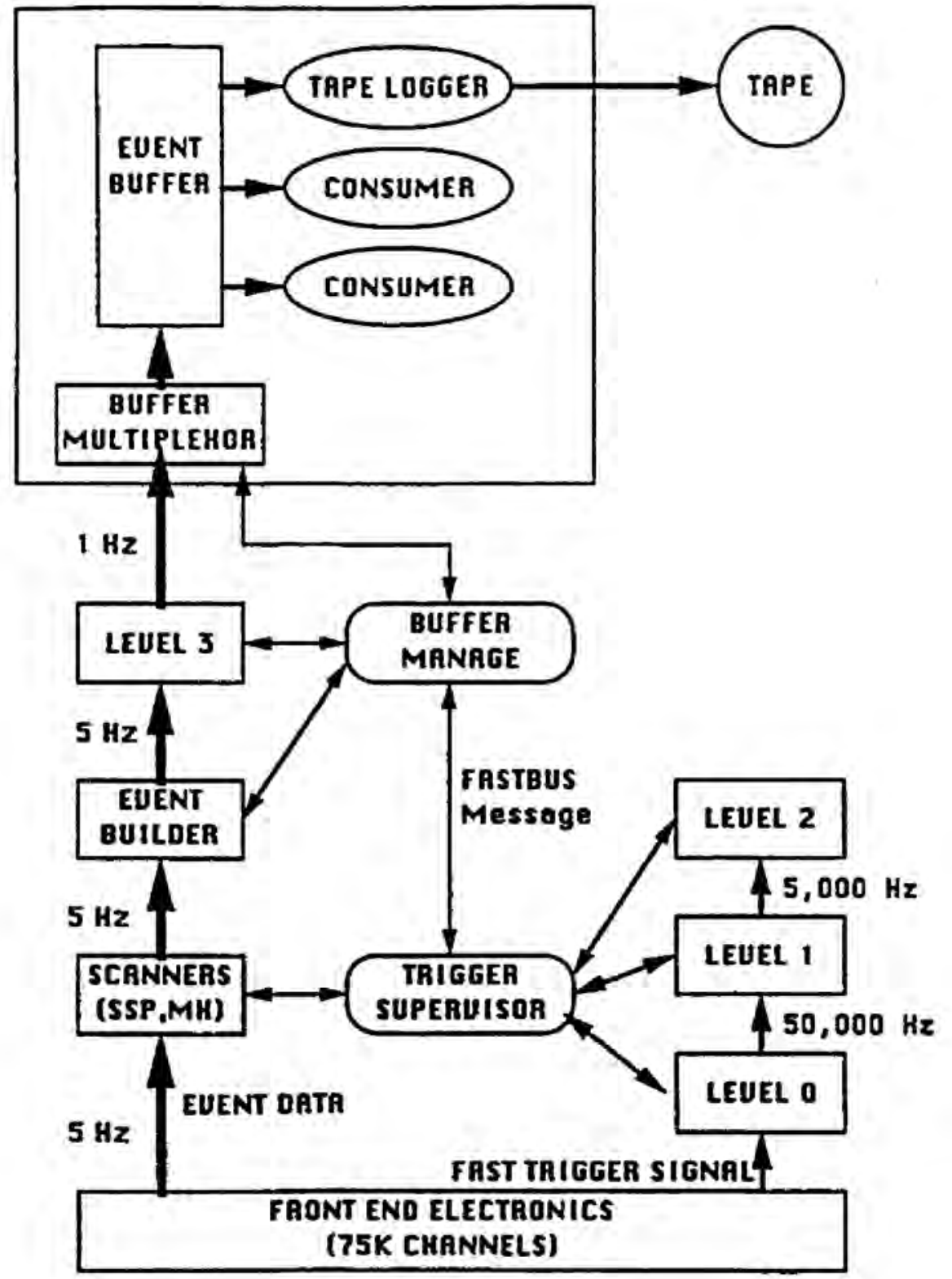

Figure 2.9: The data processing flow chart. 


\section{Chapter 3}

\section{Data Collection and Reconstruction}

To reconstruct an event in terms of leptons and quark/gluon jets, we start with the raw data collected in terms of electronic charge-counter (ADC) or time-counter (TDC) units. These raw signals must go through a process of calibration and noise removal. Pattern recognition algorithms are then used to reconstruct the electrons, muons, and jets in each event. Because we are interested in events where a $Z$ boson is produced and then subsequently decays into a pair of leptons, only a subset of the data is retained for further study. In this Chapter, we describe the data collection, reconstruction, and reduction processes.

\subsection{The 1988-89 Data Collection Period}

The 1988-89 data run started in June 1988. The CDF detector was rolled into the collision hall and checked, and the Tevatron performance in collider mode was fine-tuned. The first good data sample was written to tape in August 1988. During the run, the CDF experiment was carried out by teams, 4 persons each, that took 8 -hour shifts, 24 hours a day. The main responsibility of the shift personnel consisted of data collection, detector calibration, and detector performance monitoring. By the end of the run in May 1989, the Tevatron delivered a total of $\approx 10 \mathrm{pb}^{-1}$ of data. The CDF experiment recorded $\approx 4.7 \mathrm{pb}^{-1}$ of data on tape. The performance of the accelerator and the CDF is plotted in Figure 3.1 in terms of the integrated luminosity delivered and recorded for each week. 


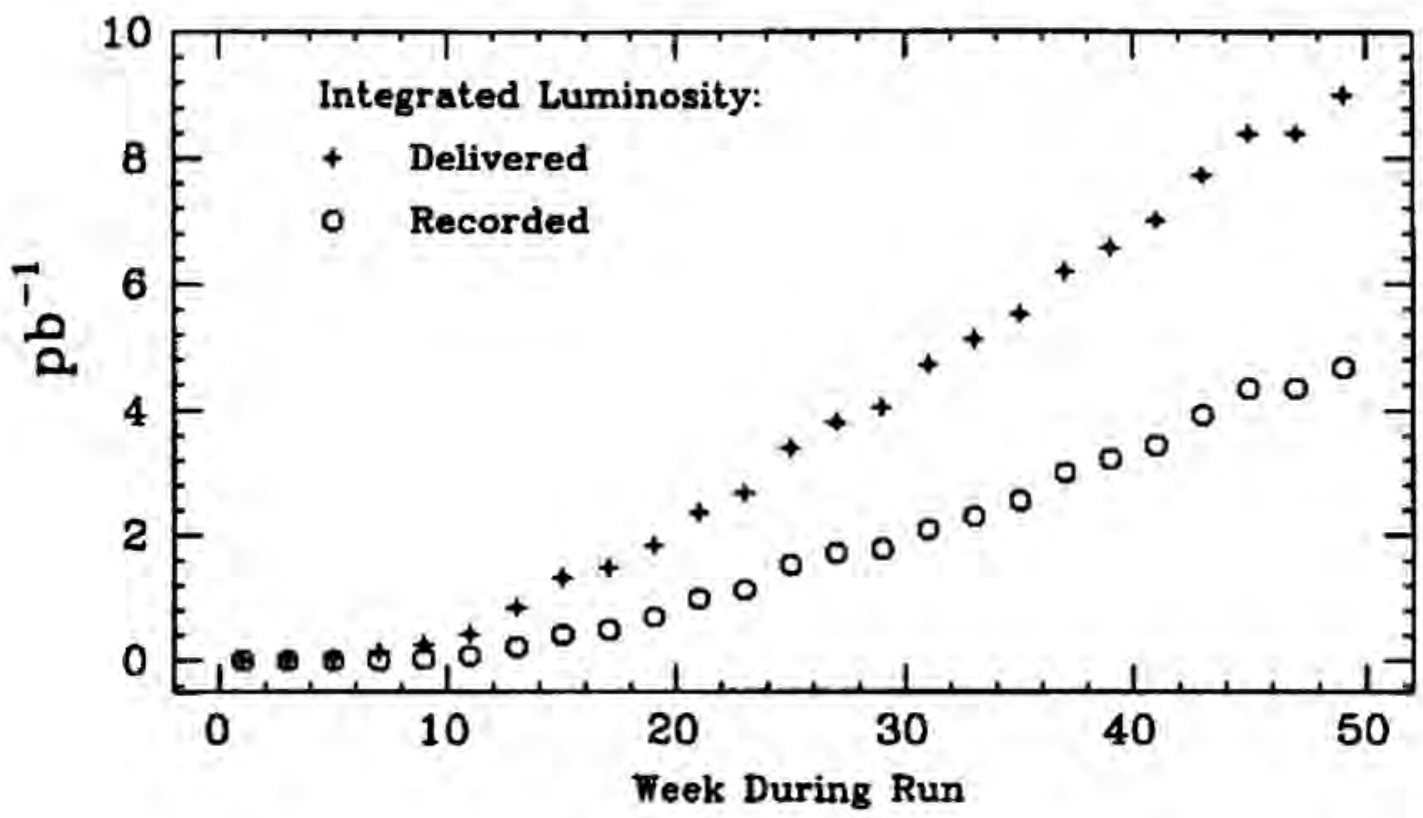

Figure 3.1: The CDF and Tevatron performance.

\subsection{Data Reconstruction}

As discussed in Section 2.2, each detector component is equipped with an online calibration and monitoring system. Variations in electronic gains and baseline reference levels (pedestal shifts) are corrected as the data is being read out. In offline data processing, a single scale factor obtained at the test beam for each calorimeter is applied to convert charge ADC counts into energy in GeV, For the muon drift chambers, the TDC counts converted into drift times, together with the ADC counts converted into charge depositions allow the muon hit position to be measured. For the CTC, the hits recorded by each wire, as determined by the TDC counters, allow charged tracks to be reconstructed.

The event vertex, the position inside the detector at which the collision took place, is determined by VTPC tracks extrapolated to the beam axis. Some events have multiple interactions and thus multiple vertices. (At an interaction rate of $50 \mathrm{kHz}, \approx 9 \%$ of the beam crossings have multiple interactions.) The primary vertex is identified as the one with the largest number of $r \cdot z$ tracks intersecting the beam axis. In the $r-\phi$ plane, the 
beam position is slightly off center relative to the CDF. Along the beam axis, the event $z$-vertex has an approximate Gaussian distribution with a $\sigma$ of $30 \mathrm{~cm}$. The $z$-vertex is important in calculating quantities such as transverse energy and in determining the overall event topology.

For the calorimeter data, spurious signals from the following sources must first be removed:

- Some electronic channels have large pedestal shifts during data taking, which would be mistaken as energy deposition in offline analysis. These large shifts are corrected using minimum bias data, taken with a trigger requiring only beam-beam counter coincidence.

- Some electronic channels have excessive noise, as indicated by a large width in the pedestal distribution. There are also dead channels, as indicated by charge injection calibrations. These bad channels are listed and kept track of using minimum bias data, and are suppressed in offline analysis.

- In some events, one of the two photo-multipliers reading out the scintillators in the CEM, CHA, and WHA would report a large amount of energy while the other would report almost no energy. This is either due to Cherenkov light from particles showering in the lightguides, or HV discharges in one of the phototubes. The signature is clear, and the fake energy is easily corrected for.

- The Tevatron Main ring passes over the CDF detector. Those events that have energy deposition from particles coming from the Main ring are filtered out using timing information from the central and endwall hadron calorimeters. The same filter also removes events with cosmic rays.

- In the plug and forward hadron calorimeters, electronic noise from ground loops in the cables that carry signals from the calorimeter pads to the front-end appear as energy in the group of towers corresponding to a ribbon cable. This noise is recognized as 
a rectangular patch in the $\eta-\phi$ "lego" plots of calorimeter energy deposition. It is eliminated from the event.

- Other spurious signals in the gas calorimeters are characterized by localized energy deposition in the longitudinal segmentation. For the PEM, HV discharges occur at the outer edges, resulting in a large signal in a single tower depth segment that matches the wire signal in a single layer. Another source of "gas spikes" is believed to be energy deposited in a single layer by heavily jonizing protons which result from slow neutrons interacting with free protons in the gas or other detector material. The gas calorimeter energy calibration constants are large because of the small sampling fraction. When the proton ranges out in a single layer, its energy deposition is scaled up by some large factor. This results in an apparently large energy deposition. These anomalous energy depositions, called "Texas Towers", are removed.[20]

The data is now prepared for full event reconstruction as discussed below. A completely reconstructed $Z$ event is shown in Figures 3.2 and 3.3, as viewed in the calorimeter $\eta$ $\phi$ geometry and in the central tracking chamber, respectively. The two narrow energy clusters with high transverse energy are the two electrons. The third broader energy cluster is the recoil jet produced along with the $Z$ in the same event. The tracking data, viewed along the beam axis, shows two stiff tracks matching the two high $E_{T}$ energy clusters for the two electrons. It is clear that the $Z p_{T}$ vector reconstructed from these two electrons is opposite to that of the jet, shown here as a cluster of charged tracks. Other low momentum tracks in the event can also be seen.

\subsubsection{Tracking}

The central tracking chamber is described in detail in Section 2.2.1. The measurement of charged tracks is important in charged lepton momentum and position determinations, as well as lepton identification. It consists of a pattern recognition procedure that finds all the hits that belong to a track, and a fitting procedure that finds the track curvature, and thus 


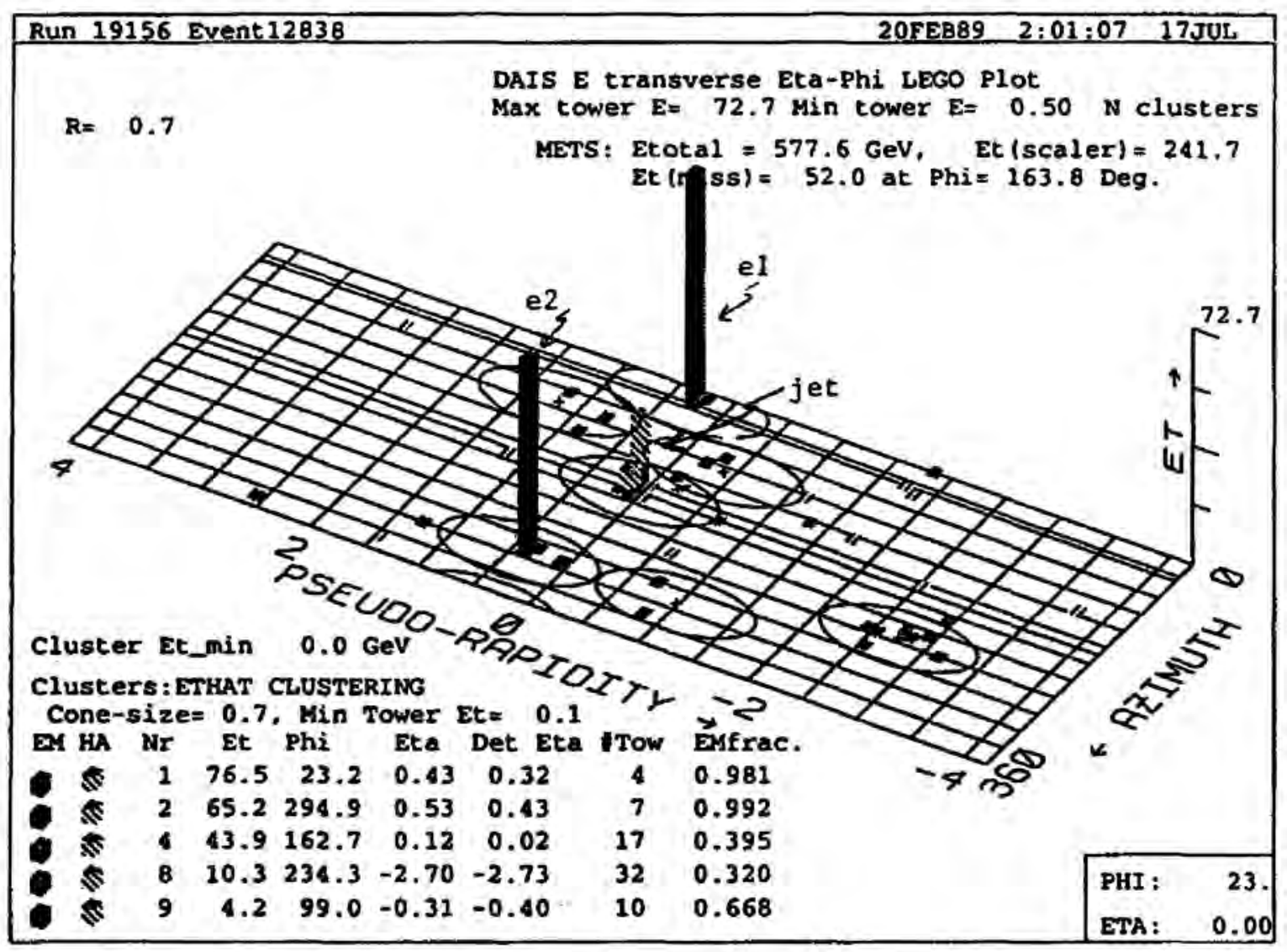

Figure 3.2: A $Z \rightarrow e \epsilon$ event observed in the CDF detector: the "lego" view of calorimeter transverse energy depositions in the $\eta-\phi$ geometry. Solid and cross-hatched towers indicate EM and hadronic energy depositions, respectively. 


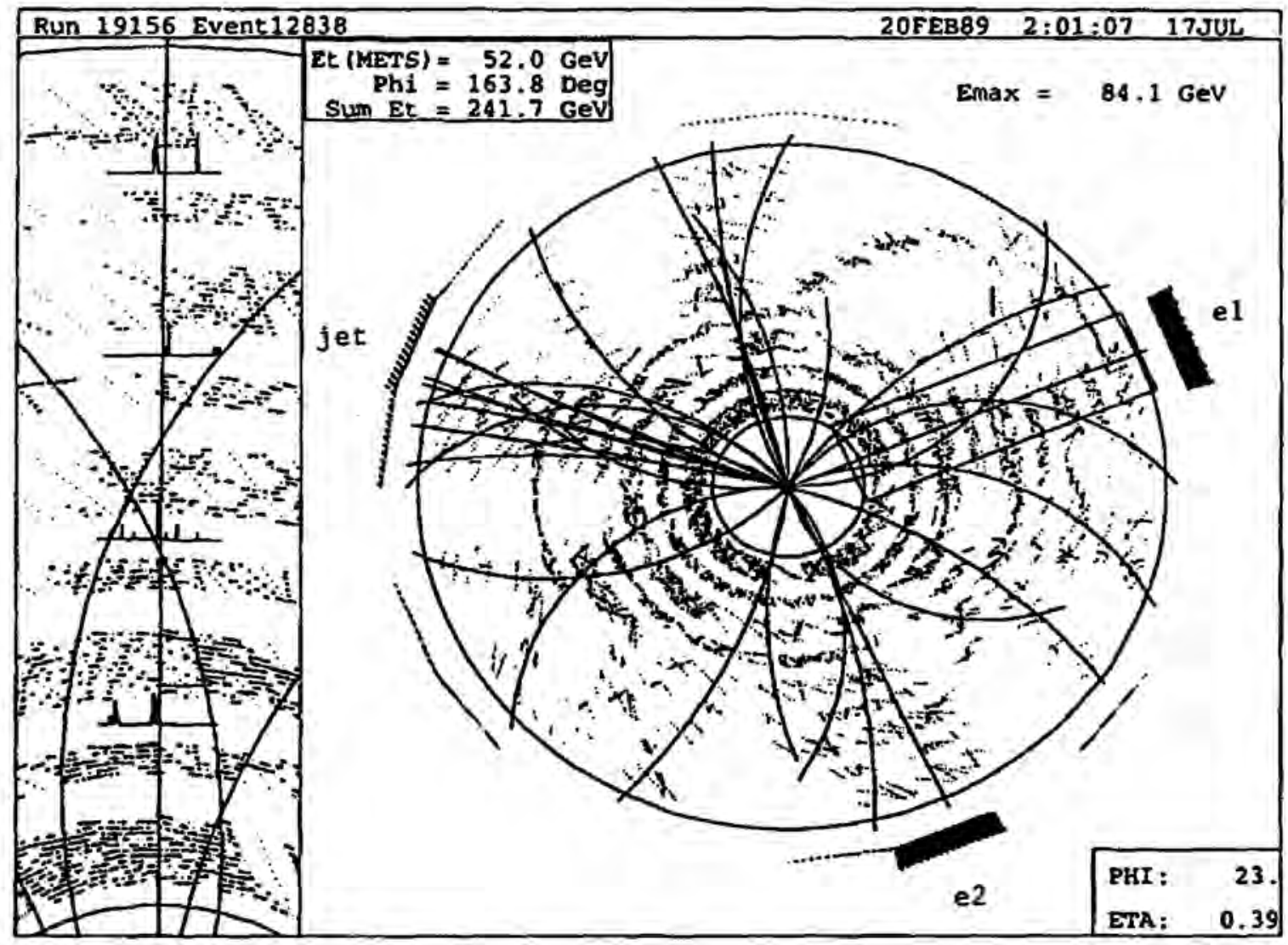

Figure 3.3: The $Z$ event shown in the previous plot viewed along the beam axis in the CTC. The calorimeter energy depositions are shown outside the CTC. 
the track momentum, from these hits. The tracking algorithm [21] is an iterative procedure:

1) First, a seed track segment in $r-\phi$ is found in a cell in the outer-most superlayer. This can be accomplished by looking for a set of at least 5 hits from each wire plane with decreasing TDC counts. The wire closest to the track would give the smallest TDC count, or the shortest drift time. The next closest wire would give a larger TDC count, and so on. The search continues in neighboring cells until all sense wires have been tested. If the search is successful, a track segment is formed using these hits.

2) Using this track segment and the beam position, a circle is calculated in $r-\phi$ corresponding to the expected trajectory of the track.

3) Hits are sought for along the circle. If more hits are found, all hits are fit to a circle without using the beam position. Hits that contribute significantly to the residuals in each superlayer are removed. Hits that are kept are marked and will not be used again in another seed track segment. If other seed tracks are found, the marked hits could be used again.

4) After step 3) of "editing hits", steps 1) to 3) are repeated to find the best set of two-dimensional hits.

5) Having found a set of hits that formed a track in the $r-\phi$ plane, the $r-\phi$ circle is projected onto the stereo wire cells. The stereo wires are tilted by $\pm 3^{\circ}$ with respect to the beam axis. That is, the position of a given stereo wire at one end of the CTC is offset in $\phi$ from the position of the same wire at the opposite end of the CTC. The offset is such that the line joining the two positions is at $3^{\circ}$ with respect to the plane formed by the beam axis and the wire position at one end. Hits from the intersected stereo wire cells are used to determine the $z$ position of the tracks. Steps 1) to 5) are iterated to find the best set of three-dimensional hits. The hits thus obtained are fit to a helix, the trajectory of a charged particle traveling in an uniform axial magnetic field. The track fitting procedure adjusts the track parameters, the 
azimuthal position $\phi$, the axial position $z$, and the radius $r$, to minimize the $\chi^{2}$ between the expected and the measured drift distance. To find the best fit, various iterative corrections are made to the drift constants, the beam position, and the chamber wire positions (alignment). [22]

\section{Drift Constants}

The raw times measured by the CTC multi-hit TDCs are corrected for:

- Channel and time dependent pedestal fluctuations.

1. The TDC channel pedestal variations due to different cable lengths and TDC offsets are corrected using pulser calibrations.

2. The TDC channel pedestal variations due to the difference in pre-amplifier responses to calibration and chamber pulses are corrected using a large sample of tracks from minimum bias data.

3. The time dependent pedestals due to variations in delays in the electronic circuits are corrected using minimum bias data for each superlayer for each run.

- Pulse width-slewing. The observed variation of the average error (measurement prediction) with pulse width and drift time are removed for each superlayer for each run.

- Drift velocity variation with position. This is due to distortions in the drift trajectory near the boundary of the drift region. (See Figure 2.5.) The correction is measured for each superlayer from minimum bias data. Using an average drift velocity for each superlayer, and accounting for variation in interaction time for events of multiple interactions, a conversion table from drift time to drift distance is obtained as a function of position. This is done whenever the chamber high voltage settings are changed.

\section{Beam Position}

The beam position can be measured accurately using VTPC information. Beam-constraining 
the track in the fitting process increases the accuracy of the fit. It is measured for each Tevatron store using a large number of tracks from minimum bias data. The lateral beam profile, from accelerator parameters, is a gaussian with $\sigma \approx 50 \mu \mathrm{m}$. The beam center is measured, allowing the beam to be off center and tilted relative to the CTC, to better than $5 \mu \mathrm{m}$. The beam position is found to vary little between each store. Using tracks passing within $1 \mathrm{~mm}$ of the beam position from the previous store removes degradation in the resolution due to secondary tracks.

\section{Alignment}

The random alignment errors in each wire are measured, again using tracks from minimum bias data, by the average discrepancy between the measured and the predicted drift distance. It is typically $<10 \mu \mathrm{m}$, consistent with the chamber design. This error does not account for systematic layer-by-layer azimuthal alignment problems. These result from a twist of the CTC endplates with respect to each other because of different mechanical stresses. To obtain corrections for the layer mis-alignment problem, more constraints are needed in the track fitting process. Otherwise, the error is compensated by adjustments in the drift model parameters. The available constraints are the beam position, with the $z$ position given by the VTPC, and the energy of the track measured by the calorimeter. The alignment procedure exploits the fact that the error on the track curvature is charge dependent, but the calorimeter response is charge independent. The $W \rightarrow e \nu$ sample provides the best source of high $p_{T}$ tracks whose energy can be measured with good resolution by the central EM calorimeter. The corrections [22] are obtained in an iterative procedure:

1. The electron track is fit with the best alignment corrections from previous iterations; and the errors in the impact parameter is plotted against azimuthal angle.

2. The track is now refit with two constraints:

- The beam position. The $z$-vertex is given by the VTPC, and the impact parameter is given by the best estimate from the previous iteration. 
- The curvature. It is fixed to be $\pm \beta / E_{T}$, where $E_{T}$ is the electron/positron transverse energy determined by the calorimeter and the $\theta$ of the track, and $\beta$ is the factor, estimated from the previous iteration, that makes the distribution $E_{T} / p_{T}$ peak at 1 .

The residuals of the fit are kept for each layer and for each charge.

3. Steps 1) and 2) are repeated for each event. Then,

- a new beam position is determined from results of 1 );

- a new estimate of $\beta$ is obtained from the $E_{T} / p_{T}$ distribution; and

- a new alignment correction is obtained from the residuals of step 2) for each layer and each charge. The results for the two charges are averaged to cancel effects from other calibration errors.

The process is repeated until the adjustment in the wire positions is small compare to the statistical error. The result is that the average $E / p$ for electrons and positrons are now the same. It is also important to note that the procedure does not depend on the absolute scale of the calorimeter, but only that its response to positives and negatives are the same.

The effect of the layer mis-alignment, as well as the correction for it, can be seen in cosmic ray data. For a cosmic ray passing through the detector near the beam axis, the in-coming and out-going branches appear as two tracks of the same momentum but opposite curvature. The distribution of cosmic rays curvature match reveals that the charge dependent shift in the curvature is removed after alignment corrections. The charge asymmetry in momentum measurement for a $35 \mathrm{GeV} / c$ track is $3 \%$ before alignment, but improved to $0.3 \%$ after alignment corrections. For measurements that average over charge, such as the $Z d \sigma / d p_{T}$ measurement, the alignment improves the momentum resolution but does not affect the momentum scale.

\section{Systematic Errors in the Track $p_{T}$ Measurement}

The curvature from the track fitting, along with the value of 1.4116 Tesla for the magnetic 
field, determines the momentum of the track. The momentum scale of the track can be set accurately by surveying the nominal wire locations with $50 \mu \mathrm{m}$ precision, and mapping the absolute magnetic field to $\pm 0.05 \%$. The uncertainty in the field is mostly due to the solenoid operating at a current of $4650 \mathrm{~A}$, whereas it was mapped at $5000 \mathrm{~A}$. [23]

Error in the curvature can be caused by one of the following:

- The beam position. Error in the interaction point introduces an error in the curvature. One term in this error averages to zero over azimuth. The other term contributes an offset to the curvature but is eliminated by the alignment procedure.

- The drift constants. Error in the drift constants leads to an error in the drift time. This is equivalent to an error on the wire position except for the drift time and direction dependence - it does not contribute any systematic error when averaged over all events.

- The wire positions. An error in the wire radial position leads to an error in the wire azimuthal position that is curvature dependent: $\Delta \phi=(d \phi / d r) \Delta r=$ curvature $\times \Delta r$. This introduces a curvature dependence in the azimuthal offset alignment. It results in a scale error.

The offset error in the curvature is limited to less than $10^{-7} \mathrm{~cm}^{-1}$ by the statistics of the $W \rightarrow e \nu$ sample used in the alignment. The scale error can be determined from a large clean sample of $J / \psi \rightarrow \mu \mu$ and $\Upsilon \rightarrow \mu \mu$ events. The fit $J / \psi$ mass agrees with the world average to within $0.03 \%$. The fit $\Upsilon$ mass is $0.1 \pm 0.1 \%$ higher than the world average. No adjustment to the momentum scale is done. An overall momentum scale uncertainty of $0.1 \%$ is determined.[4] This applies to tracks of $5 \mathrm{GeV} / c p_{T}$. And it improves at higher $p_{T}$ because the curvature is better measured at higher $p_{T}$. The track momentum resolution is improved by constraining the track to come from the interaction point. It is $\left|\delta\left(\frac{1}{p_{T}}\right)\right|=0,0011$, with $p_{T}$ in $\mathrm{GeV} / \mathrm{c}$, or, $\delta p_{T} / p_{T}=0.0011 p_{T}$. 
Table 3.1: Parameters in the offline EM clustering algorithm

\begin{tabular}{c|c}
\hline Parameter Description & Default Parameter Value \\
\hline Seed tower $E_{T}$ threshold & $3.0 \mathrm{GeV}$ \\
Daughter tower $E_{T}$ threshold & $0.1 \mathrm{GeV}$ \\
Max. daughter to seed tower $E_{T}$ & 1.0 \\
Minimum cluster $E_{T}$ & $5.0 \mathrm{GeV}$ \\
Max. Had $/$ EM ratio & 0.125 \\
CEM cluster size limit in $\eta \times \phi$ & $0.3 \times 0.3(3 \times 1$ towers $)$ \\
PEM cluster size limit in $\eta \times \phi$ & $0.5 \times 0.4(5 \times 5$ towers $)$ \\
FEM cluster size limit in $\eta \times \phi$ & $0.7 \times 0.6(7 \times 7$ towers $)$ \\
\hline
\end{tabular}

\subsubsection{The Electrons}

The reconstruction of electrons uses the fact that electromagnetic showers have a limited size, and therefore the deposited energy appears as a cluster in the calorimeter $\eta-\phi$ tower segmentation. The transverse energy, $E_{T}=E \sin \theta$, is used in cluster finding, where $\theta$ is the polar angle at the tower centroid measured from the event $z$-vertex. A list of towers with $E_{T}$ above a threshold and bordering a seed tower is formed. Towers are added to the cluster until it reaches a size limit or the list of towers is exhausted. Requirements in the clustering algorithm are summarized in Table 3.1 for the different detector components. The electron energy is measured by the EM calorimeter. The direction of electrons in the central region is measured by the beam-constrained CTC track. The direction of electrons in the plug region with strip chamber coverage is given by the shower position measured with the strips. For electrons in the plug region not covered by strips, and for electrons in the forward region, the electron direction is given by the cluster centroid measured with the pads. The electron energy and direction define the electron momentum vector. The identification of an EM cluster as an electron for this measurement is the subject of Section 4.1.

\section{Electron Energy Corrections}

The calorimeter response to electrons is obtained at the test beam. An absolute energy scale calibration is obtained using CTC momentum measurement. The electron energy is corrected for measurement errors due to cracks, edge effects, and non-linearity in the the 


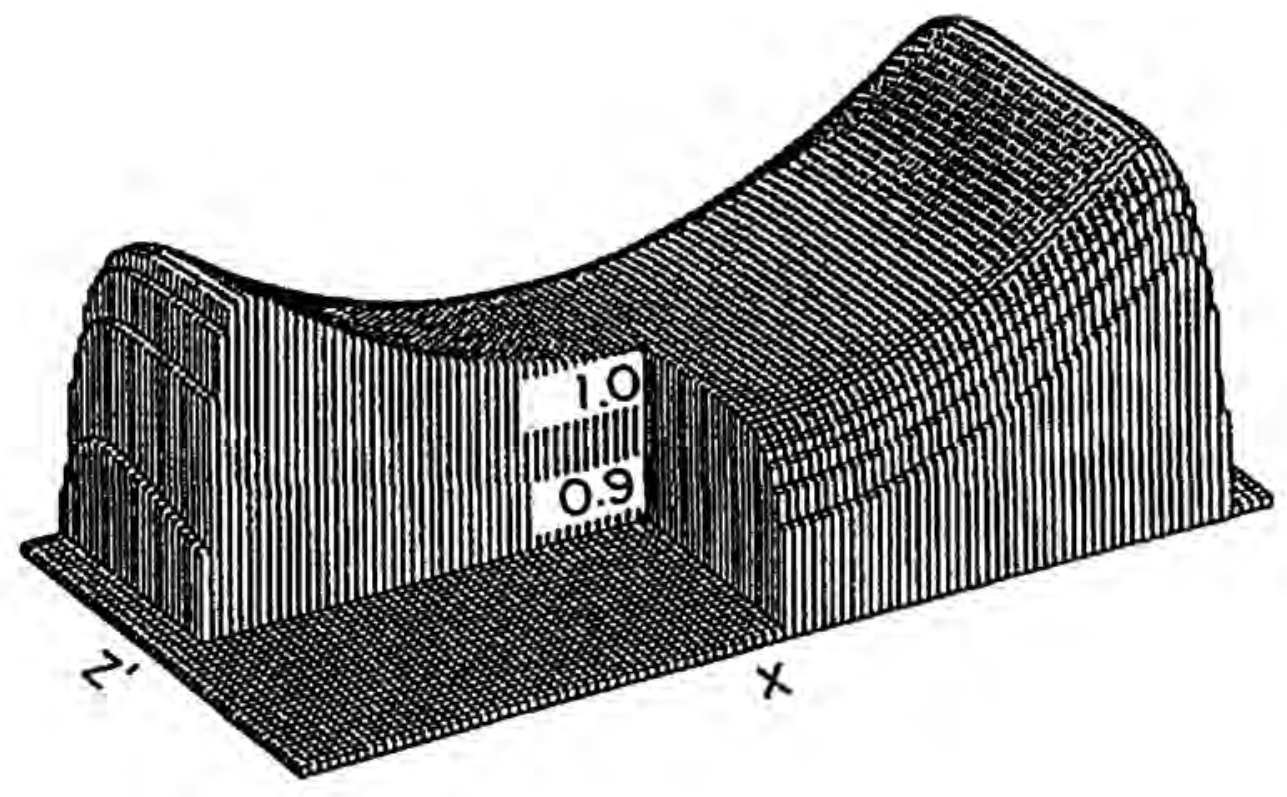

Figure 3.4: The CEM tower energy response function in local $x$ and $z$ coordinates.

calorimeter. These corrections are derived from both test beam and real $\bar{p} p$ data.

For the CEM, the following corrections are applied:

- A response map for each tower. This corrects for scintillator light collection efficiency and shower leakage dependency on the electron impact point at the face of the tower.[24] The energy scale is established at the test beam with $50 \mathrm{GeV}$ electrons at the center of the tower. The response map provides a relative correction with respect to the center of the tower. The map was obtained at the test beam by scanning each tower of the CEM with $50 \mathrm{GeV}$ electrons in $4 \mathrm{~cm}$ steps in both the $x$ and the $z$ directions. (The tower size is $\approx 23 \mathrm{~cm}$ in $x$ and $24 \mathrm{~cm}$ in $z$.) The result is parametrized as a function of $x$ and $z$. A typical tower response function is shown in Figure 3.4.

- A relative tower-to-tower response correction. It is obtained with a sample of 17000 inclusive electrons. The average $E / p$ measured by each tower is required to be the 
same. The spread in these single-tower corrections is $\approx 2.5 \%$.

- An absolute energy scale calibration with respect to the CTC momentum scale. This is obtained with a sample $W \rightarrow e \nu$ electrons. We compare the $E / p$ distribution of these electrons to a prediction from Monte Carlo simulation including radiative effects. The energy scale factor is 1.0194 . The uncertainty on the energy scale is $\pm 0.4 \%$.

For the PEM, the following corrections are applied:

- A relative tower-to-tower response correction. Each tower of the PEM was calibrated by $100 \mathrm{GeV}$ electrons at the test beam.

- Dead layer correction. Some chambers did not hold high voltage during a given run. This information is recorded in a data base. The energy not measured because of this dead layer is later accounted for by interpolation, using an average longitudinal shower profile for test beam electrons.

- Non-linear response. The energy dependence of the PEM response is obtained at the test beam.

- A relative quadrant-to-quadrant response correction.[25] After applying all known corrections listed above, a final response correction and an overall energy scale factor is obtained using electrons from $W$ and $Z$ decays. A $Z$ mass distribution, with one electron in the CEM, is formed for each PEM quadrant where another electron is found. A response correction is obtained for each quadrant with respect to the quadrant best calibrated at the test beam. The average C-P $Z$ mass for this quadrant is in good agreement with the C-C $Z$ mass. Thus, this correction also serves as an absolute energy scale calibration factor. The final correction factors are obtained through an iterative procedure, until the correction is less than $0.5 \%$. The uncertainty on the energy scale is $\pm 5 \%$, dominated by the statistics of the C-P $Z$ events in each quadrant. 
For the FEM, the following corrections are applied:

- A relative quadrant-to-quadrant response correction.[26] Response variations are obtained from the azimuthal energy flow of neutron-induced "gas spikes", or "Texas Towers". (See Section 3.2.) The rate of "Texas Towers" should be independent of azimuth. Because the "Texas Towers" energy spectrum is rapidly changing, energy scale difference between each quadrant would result in an azimuthal dependence in the rate.

- Non-linear response. The energy dependence of the FEM response is obtained at the test beam, up to a beam energy of $200 \mathrm{GeV}$. But the electron energy in the forward region from an $Z$ decay can be as high as $400 \mathrm{GeV}$. Non-linearity is measured by using the average C-F $Z$ mass to extrapolate the test beam data to higher energy.[27] By constraining to the C-C $Z$ mass, an absolute energy scale calibration factor is obtained. The uncertainty on the energy scale is $\pm 5 \%$, dominated by the statistics of the C-F $Z$ events.

\subsubsection{The Muons}

The reconstruction of muons uses the fact that a muon can penetrate a large amount of material without significant change in its momentum. The muon drift chambers are located behind $\approx 6$ hadronic absorption length of calorimeter material. The muon detector is described in Section 2.2.3. Hits in four radially aligned drift cells, one from each layer, form a muon track segment, or muon stub. The drift velocity is monitored by the drift time difference measured by sense wires offset by $2 \mathrm{~mm}$ in alternate layers. The muon hit position in $\phi$ is measured by the drift time recorded by one pair of wires from alternate layers, while the other pair resolves the left-right ambiguity. The muon hit position in $z$ is measured by the division of charge at the two ends of a sense wire. The hits in a stub are then fit to a straight line, and the muon position at the bottom layer is determined. This position is used to match an extrapolated CTC track. For the analysis of $Z \rightarrow \mu \mu$ decays, 
one of the muons can be reconstructed by simply imposing a minimum ionizing requirement on a high $p_{r}$ CTC track. The muon identification procedure is described in Section 4.2.3.

\subsubsection{Jet Clustering}

The parton model quarks and gluons from a hard $\bar{p} p$ scattering are not directly observed experimentally. Instead, a parton fragments into a shower of particles, resulting in a cluster of energy depositions in the calorimeter. The CDF jet clustering algorithm attempts to recover the 4-vectors of the original parton.[28] It clusters together the energies of towers inside a cone of fixed radius in $\eta \cdot \phi$ in an iterative process:

1. A "pre-cluster" is formed from adjacent towers with decreasing $E_{T}$. Each tower must have at least $1 \mathrm{GeV}$ of transverse energy.

2. If the sum of the transverse energies of the towers in a "pre-cluster" is above $2 \mathrm{GeV}$, a centroid in $\eta \cdot \phi$ is computed by $E_{T}$ weighting of the tower positions. All towers with $E_{T}>0.1 \mathrm{GeV}$ within a cone in $\eta \phi$ of radius 0.7 centered on the "pre-cluster" are now added to form the cluster. The centroid of the cluster is then calculated, as the $E_{T}$-weighted average of the $\eta-\phi$ position of each tower in the cluster.

3. The cone is now centered on the cluster centroid, and all towers with $E_{T}>0.1 \mathrm{GeV}$ inside the cone are included in the cluster. A new cluster centroid is calculated with this new list of towers. The process is repeated until the tower list in the cluster remains unchanged.

4. Repeat steps 1) to 3) to find other clusters. This leads to cases where two clusters overlap, with partially identical lists of towers. If the sum $E_{T}$ of towers shared by both clusters is more than $75 \%$ of the $E_{T}$ of either cluster, the two clusters are merged. Otherwise, the clusters are separated, assigning towers in the overlapping region to the nearest cluster. This division is an iterative process, with cluster centroid recalculation using the new list of towers. The original set of overlapping towers is then again divided until the list of towers in each cluster remains unchanged. 
Table 3.2: Parameters in the offline Jet clustering algorithm

\begin{tabular}{c|c}
\hline Parameter Description & Default Parameter Value \\
\hline Cluster Cone Size & 0.7 \\
Seed tower $E_{T}$ threshold & $1.0 \mathrm{GeV}$ \\
Daughter tower $E_{T}$ threshold & $0.1 \mathrm{GeV}$ \\
Minimum fraction of $E_{T}$ overlap to \\
merge two clusters
\end{tabular}

The default jet clustering parameters are summarized in Table 3.2. The cluster 4-momentum is obtained as the sum of the 4-momenta measured in each tower in the cluster, assuming the tower energy is deposited by a zero mass particle. To interpret a jet cluster as a quark or gluon requires a reasonable model of how partons turn into showers of particles, and how our detector would respond to these particles. The Monte Carlo simulation of jet fragmentation and energy response are described in Chapter 5.

\subsection{The Data Sample}

The complete data sample collected during the 1988-89 run consists of events passing various triggers. For example, events obtained with the jet triggers requiring a cluster above some $E_{T}$ threshold are suitable for QCD jet measurements, while events obtained with the electron triggers requiring an EM cluster above some $E_{T}$ threshold are suitable for electroweak measurements. The $Z$ boson events used in this measurement are observed in their decays $Z \rightarrow e e$ and $Z \rightarrow \mu j$. These events are characterized by a pair of energetic electrons or muons. The data sample used in this measurement consists of events that have at least one high transverse momentum charged lepton ( $e$ or $\mu$ ).

Furthermore, for a good absolute cross section measurement, we use data collected under uniform conditions throughout the run. We remove from the sample data collected under the following anomalous conditions.

- Bad-quality runs (this amounts to $\approx 5 \%$ of the data): 
1. runs that have abnormally high trigger rates, as measured with minimum bias events;

2. runs in which a number of the channels in the plug EM calorimeter are not read out; and

3. runs that have large means or sigmas in the missing $E_{T}$ distribution, as measured with minimum bias events.

- Bad muon trigger runs (this amounts to $\approx 14 \%$ of the muon data) in which the muon trigger hardware had problems.

\subsection{The Integrated Luminosity}

The luminosity is calculated using the collision rate and live time information recorded for each event by the beam-beam counters (BBC), and an estimate of the component of the total $\tilde{p} p$ cross-section seen by the BBC:

$$
\mathcal{C}=\frac{\boldsymbol{R}_{B B C}}{\sigma_{B A C}}
$$

A total interaction rate for each run, as well as each tape, is accumulated, using the BBC information for the event with the smallest event number and the event with the largest event number. This is not exact since events were written to tape out of order by Level 3. The rate for each run is exact except for cases where events were rejected in Level 3 after the event with highest event number was written, and for cases where one or more of the tapes was found to be unusable and the rates for each tape had to be subtracted. The collision rate and live time information were corrected for multiple interactions and event loss due to an error in the Event Builder.

The effective $\mathrm{BBC}$ cross-section $\left(\sigma_{B A C}\right)$ at $\sqrt{s}=1.8 \mathrm{TeV}$ is obtained by scaling the weighted average of $\sigma_{B B C}$ estimated from UA4 $\sigma_{t o t}$ measurement and the $\sigma_{B B C}$ derived from Tevatron beam parameters at $\sqrt{s}=0.546 \mathrm{TeV}[29]$ : 


$$
\frac{\sigma_{B B C}(1800)}{\sigma_{B B C}(546)}=\frac{\frac{R_{B B C}(1800)}{\mathcal{L}_{\text {acc }}(1800)}}{\frac{R_{B A C}(546)}{\mathcal{L}_{\text {acc }}(546)}} .
$$

The uncertainty on the luminosity measurement from accelerator parameters, $\mathcal{L}_{a c c}$, is $11 \%$, but the uncertainty on the ratio of $\mathcal{L}_{\text {acc }}$ measured at two energies is only $4.3 \%$.

The UA4 $\sigma_{t o t}$ measurement uses a luminosity independent method by measuring the total interaction rate, elastic plus inelastic simultaneously, with the extrapolation of the differential elastic scattering rate to the optical point [30]. From $\sigma_{\text {tot }}$, the effective crosssection seen by the UA4 trigger counters can be derived. This is then related to $\sigma_{B B C}$ by correcting for the slightly different geometric acceptances between the BBC and the UA4 trigger counters, and the BBC efficiency. We obtain $\sigma_{R B C}^{U A 4}=(37.1 \pm 2.1) \mathrm{mb}$.

Another estimate of $\sigma_{B A C}$ can be obtained from using accelerator parameters. The luminosity in bunched beam colliders can be calculated as:

$$
\mathcal{L}=N_{p} N_{\bar{p}} \int \frac{1}{\sqrt{2 \pi} \sigma_{z}} \frac{e^{-\frac{z^{2}}{2 \sigma_{z}^{2}}}}{4 \pi \sigma_{x}\left(z, \frac{d p}{p}\right) \sigma_{y}\left(z, \frac{d p}{p}\right)} d z .
$$

The beam parameters used are:

- the number of protons (antiprotons) in each bunch, $N_{p}\left(N_{\bar{p}}\right)$, and

- the bunch longitudinal profile, $\sigma_{z}$, measured by a current monitor;

- the bunch transverse size, $\sigma_{x}$ and $\sigma_{y}$, measured by passing wires through the beam;

- and the momentum dispersion, $\frac{d p}{p}$, derived from the longitudinal profile and the measured RF voltage.

Using the BBC coincidence rates from data collected during Tevatron running at $\sqrt{s}=$ $546 \mathrm{GeV}$, we obtain $\sigma_{A B C}^{a c c}=(32.8 \pm 3.6) \mathrm{mb}$. A weighted average of $\sigma_{A B C}^{U A 4}$ and $\sigma_{B A C}^{a c c}$ gives $\sigma_{B A C}(546)=(36.0 \pm 1.8) \mathrm{mb}$. The scaling factor, the right hand side of Equation 3.2, is measured to be $1.30 \pm 0.06$, and so $\sigma_{B B C}(1800)=(46.8 \pm 3.2) \mathrm{mb}$. The uncertainty on the integrated luminosity, dominated by the uncertainty on $\sigma_{B A C}$, is $6.8 \%$. 


\section{Chapter 4}

\section{Event Selection}

The data are reconstructed in terms of four-vectors of the leptons and jets, along with the information necessary to identify these particles. Our sample, described in Section 3.3, is selected by requiring at least one high transverse momentum lepton in the event. It contains not only $Z$ 's, but also $W$ 's, as well as backgrounds. We must apply additional selection criteria to obtain a sample of $Z$ events for the $d \sigma / d p_{T}$ measurement. In this Chapter, we discuss electron and muon identification, and the selection of $Z \rightarrow e \epsilon[31,32,33]$ and $Z \rightarrow \mu \mu[34]$ events from our data sample.

\subsection{Electron Selection}

The electromagnetic energy clustering procedure described in Section 3.2 .2 is based on the general characteristics expected from electrons in CDF, and not every EM cluster is a real electron. For example, a jet cluster would be reconstructed as an EM cluster if the fluctuations in the fragmentation process gives the jet cluster a large EM energy fraction. This is the case when the jet consists of $\pi^{0} \mathrm{~s}$ and low momentum $\pi^{ \pm} \mathrm{s}$. Thus, cuts on electron quality parameters are needed to identify good electrons.

\subsubsection{Electron Fiducial Region}

Because the electron identification and energy reconstruction rely heavily on calorimeter information, we restrict electrons to regions of the calorimeter away from the edges. This is the region where electron detection efficiency is uniform. It is also where the energy scale 
Table 4.1: The electron fiducial volume

\begin{tabular}{ccc}
\hline Region & Fiducial Cuts & Description \\
\hline CEM & $|x| \leq 21 \mathrm{~cm}$ & cluster be within $21 \mathrm{~cm}$ of the tower center \\
& $|z| \leq 9 \mathrm{~cm}$ & cluster be $9 \mathrm{~cm}$ away from the $90^{\circ}$-crack \\
& $\neq$ Tower-9 & exclude outer-most $($ in $\eta)$ CEM tower \\
& $\neq$ Chimney-Tower & exclude tower containing cryostat access \\
\hline PEM & $\neq \eta$-edge & exclude two inner $/$ outer $\eta$ tower-annuli \\
& $\neq \phi$-edge & $5^{\circ}(1$ tower $)$ away from the edge \\
& $\neq$ Bad-Tower & exclude dead PEM channels \\
\hline FEM & $|\eta|<3.7$ & exclude small pads near the beam-pipe \\
& $\neq \phi$-edge & $5^{\circ}(1$ tower $)$ away from the edge \\
\hline
\end{tabular}

calibration is reliable. The fiducial requirements are summarized in Table 4.1 for the three EM calorimeters. The EM cluster cannot have the seed tower in one of those excluded calorimeter towers. The cuts are made on the EM cluster seed tower location. For the CEM cluster position requirement, we cut on the position measured by the strip chambers, not the extrapolated CTC track.

\subsubsection{Electron Identification Cuts}

The electron identification criteria are designed to reject fake electron backgrounds from hadronic particles. The backgrounds are mainly overlapping $\pi^{ \pm} s$ and $\pi^{0} s$, or early showering $\pi^{ \pm} \mathrm{s}$ that leave large amount of energy in the EM compartment. These hadronic particles are part of the quark/gluon jet, and therefore have a large amount of energy deposited in the surrounding calorimeter towers - that is, they are mostly non-isolated. The isolation requirement would also reject real electrons, from semi-leptonic b-quark decays, for example, that are generally near or inside the $b$-quark jet.

The identification process starts with an electromagnetic (EM) cluster as defined in Section 3.2.2. Because the electron identification capabilities are different among the detector components, the electron quality parameters used are different for electron candidates found in the central, the plug, and the forward regions. Furthermore, a combination of these parameters are selected to jdentify $Z$ electrons. In particular, we select a clean sample of $Z$ 
Table 4.2: The tight central electron cuts.

\begin{tabular}{ccc}
\hline$E_{T}$ & $>$ & $20 \mathrm{GeV}$ \\
$E / p$ & $<$ & 1.5 \\
$\mathrm{LSHR}$ & $<$ & 0.2 \\
$\chi_{\text {strip }}^{2}<$ & 15 \\
$|\Delta x|$ & $<$ & $1.5 \mathrm{~cm}$ \\
$|\Delta z|$ & $<$ & $3.0 \mathrm{~cm}$ \\
Iso & $<$ & 0.1 \\
$\mathrm{Had} / \mathrm{EM}$ & $<$ & $0.055+0.045 E / 100$ \\
\hline
\end{tabular}

events by requiring one electron in the central region to pass tight cuts, allowing the second electron in any of the three regions to pass only loose requirements. The cut parameters are described in detail below. The cut values are summarized in Table 4.2 and Table 4.3. We also show the distributions of these cut parameters for the sample of $Z$ electrons used in the cut efficiency measurement. The procedures for selecting this electron sample and measuring the cut efficiency are described in Section 4.1.3. The cut parameters common for all three regions are listed below.

- $E_{\text {had }} / E_{\text {em: }}$ the ratio of the energy measured in the hadronic compartment to that measured in the EM compartment of the calorimeter. It rejects EM clusters with too much hadronic energy, such as those resulting from overlaps of charged and neutral pions.

- Iso: the measure of isolation. ${ }^{1}$ It is the amount of transverse energy deposited in a cone of radius 0.4 in $\eta-\phi$ surrounding the electron candidate normalized to the transverse energy of the electron:

$$
I \text { so }=\frac{\left(\sum_{\text {towers }}^{\text {cone }} E_{T}\right)-E_{T}^{e}}{E_{T}^{e}},
$$

\footnotetext{
The definition used in this thesis is that of Reference [31]. Alternative definition uses the total $E_{r}$ inside the cone in the denominator.[32]
} 
Table 4.3: The loose second electron cuts.

\begin{tabular}{lccc}
\hline If CEM electron: & $E_{T}$ & $>$ & $10 \mathrm{GeV}$ \\
& $E / p$ & $<$ & 2.0 \\
& Iso & $<$ & 0.1 \\
& $\mathrm{Had} / \mathrm{Em}$ & $<$ & 0.1 \\
\hline If PEM electron: & $E_{T}$ & $>$ & $10 \mathrm{GeV}$ \\
& $\chi_{\mathbf{3} \times 3}^{2}$ & $<$ & 20 \\
& VTPC occupancy & $>$ & 0.5 \\
& Iso & $<$ & 0.1 \\
& $\mathrm{Had} / \mathrm{Em}^{2}$ & $<$ & 0.1 \\
\hline If FEM electron: & $E_{T}$ & $>$ & $10 \mathrm{GeV}$ \\
& Iso & $<$ & 0.1 \\
& $\mathrm{Had} / \mathrm{Em}^{2}$ & $<$ & 0.1 \\
\hline
\end{tabular}

where the cone is centered on electron and the sum is over the transverse energies in all towers whose centroids are within that cone.

In the central region, CEM electron can be identified by using the following cuts.

- $E / p$ : the ratio of the electron energy measured by the CEM to the electron momentum measured by the tracking chamber. We use it to make an energy-momentum matching cut. It rejects high $p_{T} \pi^{ \pm}$. A $\pi^{ \pm}$would deposit small amounts of energy in the EM calorimeter because the $\pi^{ \pm}$interaction length is much longer than the electron radiation length. This ratio also rejects random overlaps of $\pi^{0} \mathrm{~s}$ with low momentum tracks. The selection cut, $E / p<1.5$, accepts electrons that have radiated a photon, and therefore lower momentum.

- $\Delta x$ and $\Delta z$; the differences between the electron position determined by the proportional strip chambers (imbedded inside the CEM) and that determined by the extrapolated CTC track, in the $x$ and $z$ directions, respectively. This position matching requirement rejects random overlaps of $\pi^{0} 8$ with $\pi^{ \pm} \mathrm{s}$ in the case where the $\pi^{0}$ (after decaying into a pair of photons) gives an EM cluster and the $\pi^{ \pm}$gives a high $p_{T}$ charged track.

- $\chi_{\text {strip: }}^{2}$ the $\chi^{2}$ between the transverse shower profile (in the $z$-view) measured by the 
strips in the central strip chambers and the expected profile determined from test beam electrons.

- Lshr: a $\chi^{2}$-like quantity that measures the lateral (in the $z$-view) sharing of energy in an EM cluster using the energies in the towers adjacent to the seed tower of the cluster and the expected amount of lateral sharing determined from test beam electrons. It is defined as

$$
L s h r=0.14 \times \sum_{k} \frac{M_{k}-P_{k}}{\sqrt{(0.14 \times \sqrt{E})^{2}+\left(\Delta P_{k}\right)^{2}}},
$$

where the sum is over towers adjacent to the seed tower, $M_{k}$ is the measured tower energy and $\boldsymbol{P}_{\boldsymbol{k}}$ is the tower energy predicted using the shower position and a shower profile obtained from test beam, $E$ is the cluster energy, and $\Delta P_{k}$ is the error in $P_{k}$ associated with a $1 \mathrm{~cm}$ error in the shower position.[35]

The shower shape requirements specified by $L s h r$ and $\chi_{\text {strip }}^{2}$ also help reject overlaps of multiple $\pi^{0} \mathrm{~s}$ and a $\pi^{ \pm}$.

The distributions of the electron isolation are shown in Figure 4.1 for each detector region. The distributions of the CEM electron selection parameters are shown in Figure 4.2. The arrows indicate where the cuts are for each selection parameter. The plots are made with a sample of $Z$ electrons nsed in the efficiency measurement. This is discussed in Section 4.1.3.

The CEM cuts described above are sufficient for selecting a sample of inclusive central electrons. The $Z$ events with both electrons in the central region are selected by requiring the second electron to pass a loose $E / p$ cut, in addition to the $E_{h a d} / E_{e m}$ and $I$ so cuts. For the $Z$ events with one electron in the central region and the second one in the forward region, the second electron in the forward region need only pass the $E_{\text {had }} / E_{\text {em }}$ and $I s o$ cuts. For those with the second electron in the plug region, the following additional cut parameters are used.

- $\chi_{3 \times 3}^{2}$ : the $\chi^{2}$ between the transverse shower profile (in both the $\phi$-view and the $\theta$ view) measured by the $8(3 \times 3$ in $\eta-\phi)$ towers surrounding the seed tower in the PEM 
and the expected profile determined from test beam electrons.

- VTPC Occupancy: the ratio of the number of wire hits found to that expected along the direction from the event vertex to the position of the electron at the PEM. A high occupancy indicates the presence of a charge track.

The distributions of the cut parameters used to identify the second electron in a $Z$ event are shown in Figure 4.3. The arrows indicate where the cuts are for each selection parameter. The plots are made with a sample of $Z$ electrons used in the efficiency measurement. To select a $Z$ event, we further impose kinematic requirements on the electrons as discussed in Section 4.5.

\subsubsection{Electron Identification Cut Efficiency}

The electron quality cut parameters are described in Section 4.1.2. The cut values are listed in Table 4.2 and Table 4.3. The efficiencies of these cuts are measured from a sample of electrons selected from the $Z$ data sample. This electron sample is selected as follows:

1. For each event, require at least one electron in the central region to satisfy the tight cuts listed in Table 4.2 .

2. The second electron is included in the sample for loose cut efficiency measurement if it

- passes the isolation cut,

- has at least $10 \mathrm{GeV}$ of $E_{T}$, and

- forms a mass between 75 and $105 \mathrm{GeV}$ with the electron selected in step 1.

3. The second electron is included in the sample for tight cut efficiency measurement if it

- is in the central region,

- passes the isolation cut, 


\section{Electron Isolation Distributions}
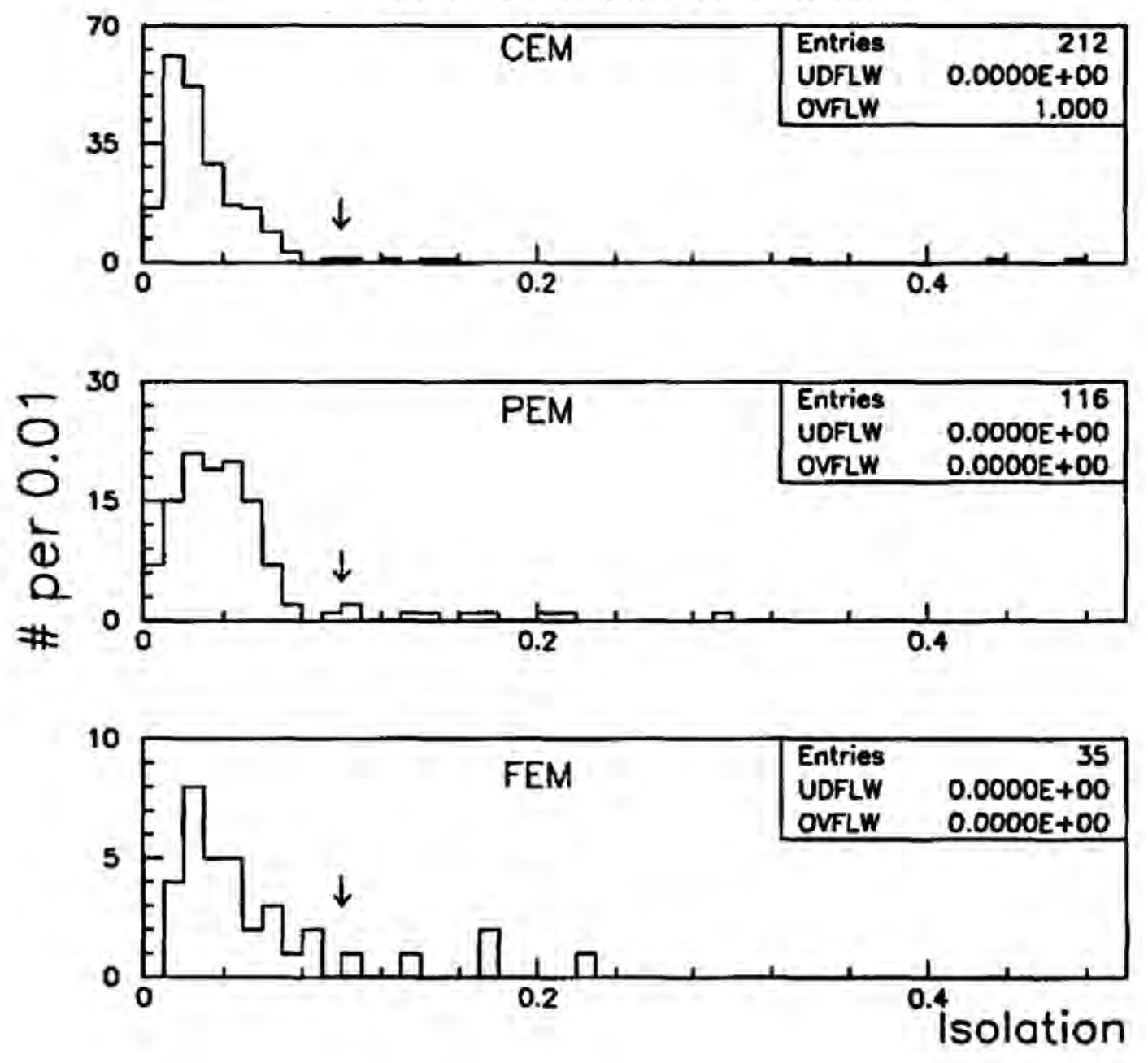

Figure 4.1: The distribution of electron isolation for each detector region. The plots are made with a sample of $Z$ electrons. 
Tight CEM Electron Cuts
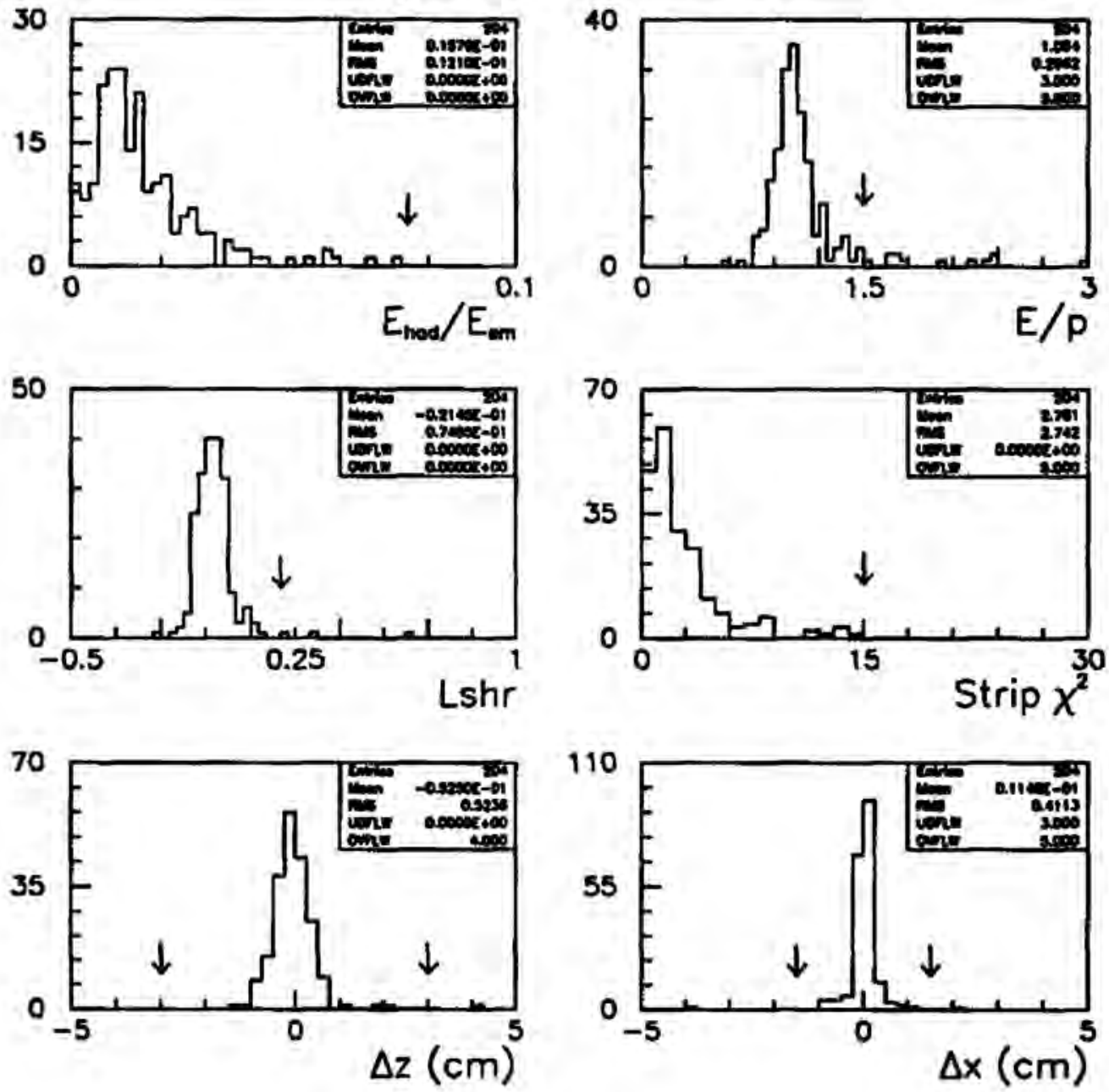

Figure 4.2: The distributions of variables used for tight CEM electron cuts from a sample of $Z$ electrons. The arrows indicate the cuts. (The $E_{\text {had }} / E_{\text {em }}$ cut depends on energy and the arrow indicates the cut value for an electron with $45 \mathrm{GeV}$ energy.) 

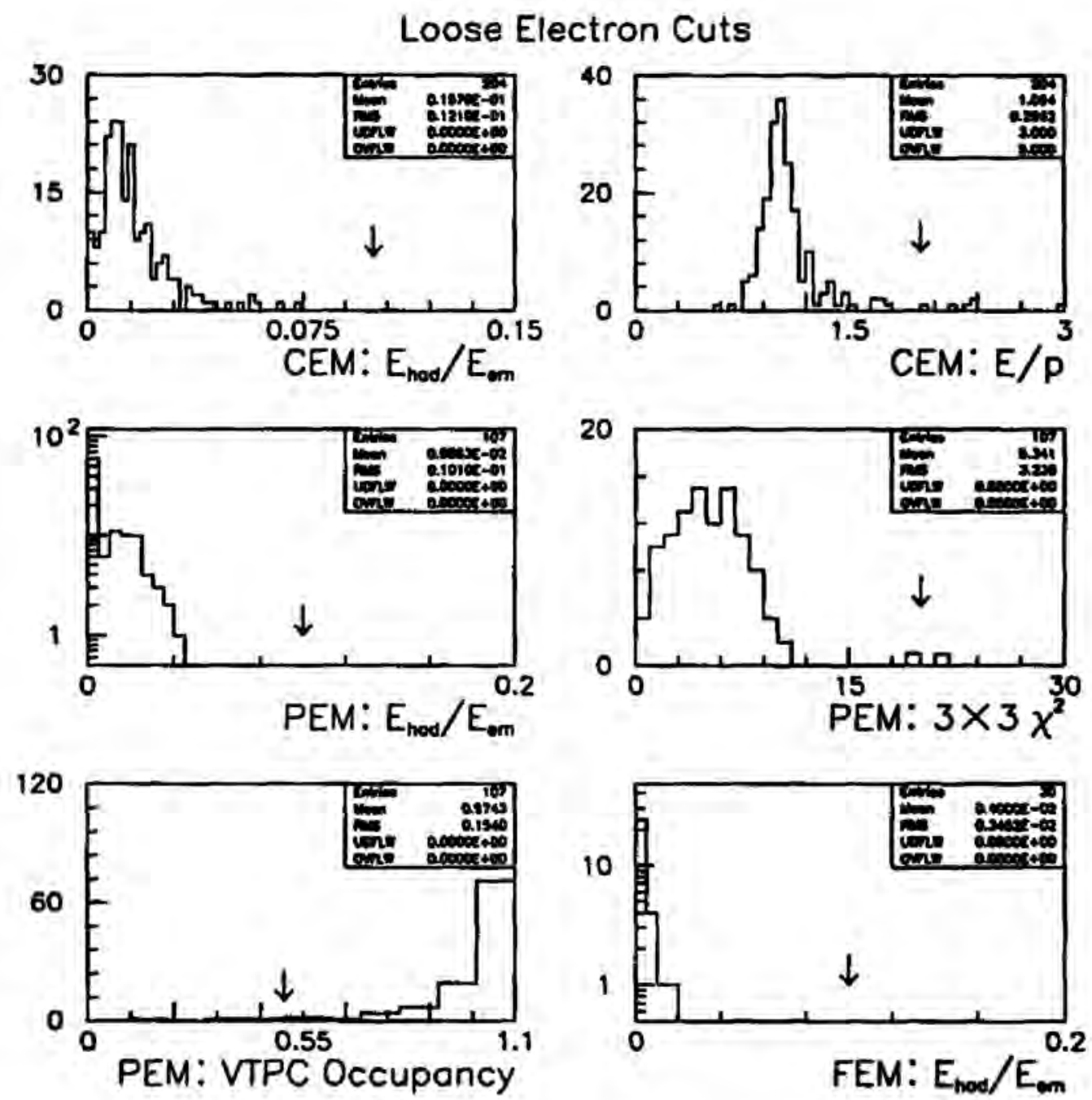

Figure 4.3: The distributions of cut variables used to identify the second electron in a $Z$ event. The arrows indicate the cuts. The plots are made with a sample of $Z$ electrons used in the efficiency measurement. 
- has at least $20 \mathrm{GeV}$ of $E_{T}$, and

- forms a mass between 75 and $105 \mathrm{GeV}$ with the electron selected in step 1.

Note that if both electrons are in the central region, both can be included in the sample for the tight cut efficiency measurement.

This sample of electrons is unbiased with respect to the selection cuts except for the isolation cut. The efficiency of the set of cuts listed in Table 4.2 and Table 4.3 is obtained from the number of electrons that passes the cuts. The efficiency of the isolation cut is obtained separately (see below), and the total electron selection efficiency is the product of the electron cut efficiency and the isolation cut efficiency. The efficiencies measured this way, and summarized in Table 4.4, are for isolated electrons.

The isolation cut is used to reduce the background in the sample. Any residual background contamination in this sample wonld imply that the efficiencies have been underestimated. However, from the isolations of the electrons in this sample, the fake electron background is estimated to be small, and a conservative estimate shows that the systematic uncertainty due to background is comparable to the statistical uncertainty.[32] We conclude that the uncertainties on the efficiencies are approximately determined by the statistics of the sample.

As a check, we measure the CEM selection cut efficiencies using a sample of $W$ electrons selected with the following cuts:

- Require a CEM cluster with $E_{T}>25 \mathrm{GeV}$ and an associated track with $p_{T}>$ $7.5 \mathrm{GeV} / c$ to pass the isolation cut.

- Cut on the missing transverse momentum: $\not_{T}>20 \mathrm{GeV}$. The $\not_{T}$ is defined as:

$$
\overrightarrow{H_{T}}=-\sum_{i} E_{T}^{i} \cdot \hat{n}_{i}
$$

a vector sum of the transverse energies in calorimeter towers in the region $|\eta|<3.6$. 
Table 4.4: The isolated electron identification cut efficiency measured from $Z$ electrons.

\begin{tabular}{lc} 
& $\epsilon_{\text {ele }}$ \\
\hline CEM tight cuts: & $0.868 \pm 0.025$ \\
CEM loose cuts: & $0.934 \pm 0.017$ \\
PEM loose cuts: & $0.947 \pm 0.023$ \\
FEM loose cuts: & $1.00_{-0.06}^{+0}$ \\
\hline
\end{tabular}

- Cut on the significance of the $\not_{T}$ measurement:

$$
\sigma\left(\not_{T}\right) \equiv \frac{\not_{T}}{\sqrt{\sum_{\text {towers }} E_{T}}}>2.5 .
$$

- Cut on the $W$ transverse mass:

$$
M_{T} \equiv \sqrt{2 E_{T} \not H_{T}\left(1-\cos \left(\phi(e)-\phi\left(\not_{T}\right)\right)\right)}>50 \mathrm{GeV} .
$$

- Cut on di-jet events: we reject events with a jet cluster with $E_{T}>10 \mathrm{GeV}$ within $30^{\circ}$ in $\phi$ opposite of the electron candidate.

The efficiencies of the CEM tight and loose cuts measured from the $W$ electrons are found to be $0.864 \pm 0.007$ and $0.947 \pm 0.004$, respectively.[32] These are in good agreement with efficiencies obtained from the $Z$ electrons.

\section{The Isolation Cut Efficiency}

The electrons from $Z$ decays carry high $p_{T}$, and have energy well above the energy deposition from the underlying event. Since isolation is a measure of the energy surrounding the electron candidate, used to reject backgrounds from QCD jets, a real $Z$ decay electron would fail the isolation cut only if there is a large upward fluctuation in the underlying event energy deposition near the electron. The underlying event energy deposition is, however, independent of the azimuth. We exploit this azimuthal symmetry to measure the electron isolation cut efficiency. The method is described below. We discuss the systematic uncertainty in the isolation cut efficiency in Section 4.3.1.

Figure 4.4 offers a graphical illustration of the method: 


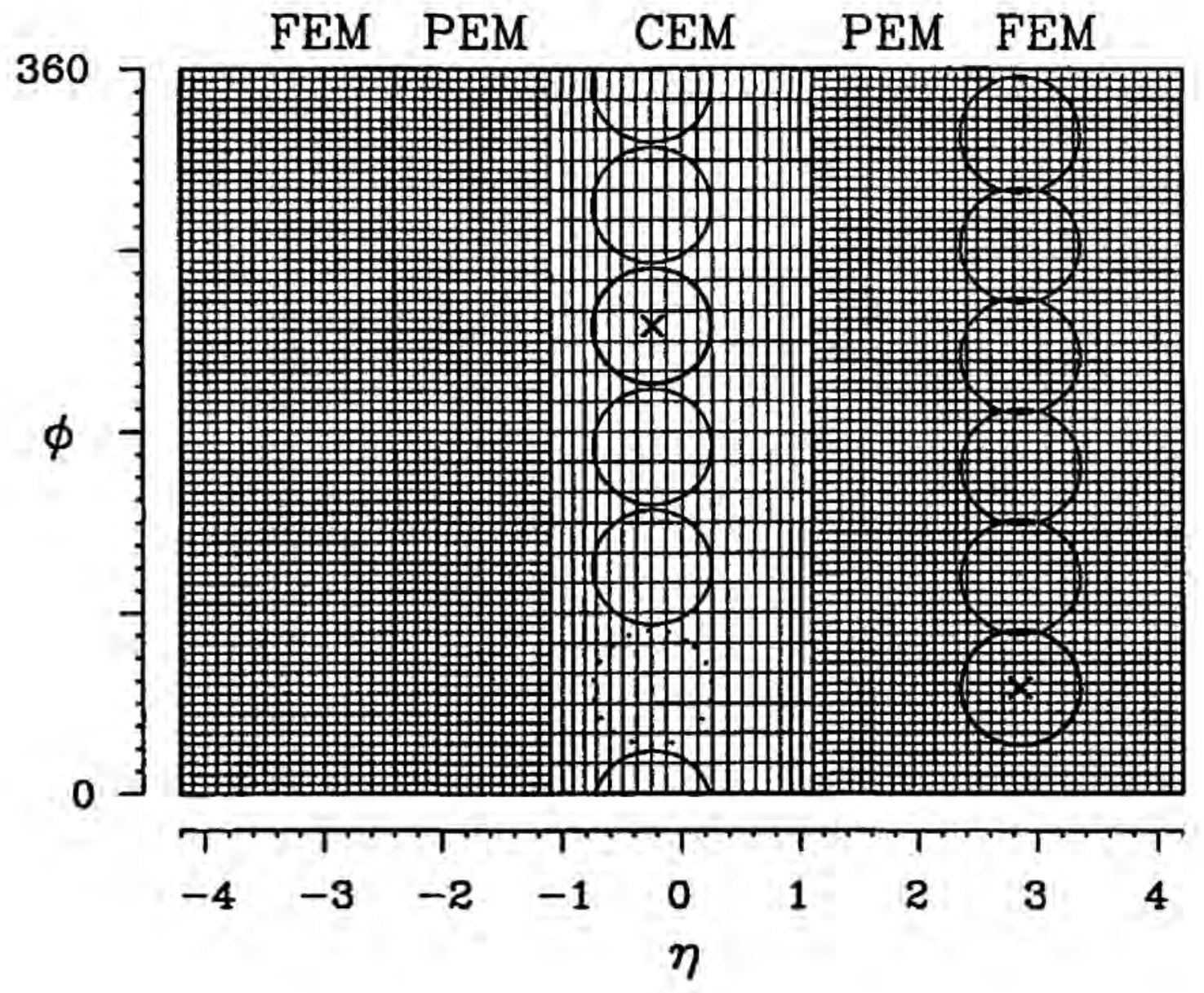

Figure 4.4: Illustration of the method nsed for finding the isolation cut efficiency. Each circle has radius of 0.4 in $\eta \phi$, representing the cones for calculating equivalent isolation. The $x$ 's mark locations of the $Z$ electrons. The dotted circle is $180^{\circ}$ away from the the electron in CEM and is not used. 
1. Starting with the $\eta \phi$ position of the electron, marked with an $\mathrm{x}$ in the figure, find a new cone of radius 0.4 at the same $\eta$ but offset in $\phi$ such that the two cones do not overlap.

2. For each new cone thus found, calculate an equivalent isolation as in Equation 4.1,

$$
I s o=\frac{\left(\sum_{\text {towers }}^{\text {cone }} E_{T}\right)-E_{T}^{\epsilon}}{E_{T}^{e}},
$$

except now in the sum over the transverse energies in the towers within the new cone, a tower that maps into a tower in the electron cluster is replaced by the tower in the electron cluster. For example, for the new cone whose center is offset by 4 towers, as is the case for the two nearest new cones in the CEM, the energy of the tower 4 towers away (in $\phi$ ) from a tower in the electron cluster is replaced by the energy of the tower in the electron cluster,

3. The next cone is selected and another equivalent isolation is calculated. The process continues until it wraps around in $\phi$.

4. A distribution of the equivalent isolation is made, from which the efficiency of the isolation cut is obtained.

The distributions of the equivalent isolation for each calorimeter are shown in Figure 4.5. The plots are made with the sample of $Z$ electrons used in the efficiency measurement. Each event contributes more than one entry. For CEM electrons, we do not use the cone that is $180^{\circ}$ away from the electron - this cone tends to have energy from the other electron that is back-to-back in $\phi$. The efficiency for the isolation cut is summarized in Table 4.5. The uncertainty is dominated by the assigned $4 \%$ systematic uncertainty, from the variation in the efficiency from each cone, as discussed in Section 4.3.1. The total electron selection efficiency is summarized in Table 4.6 . 

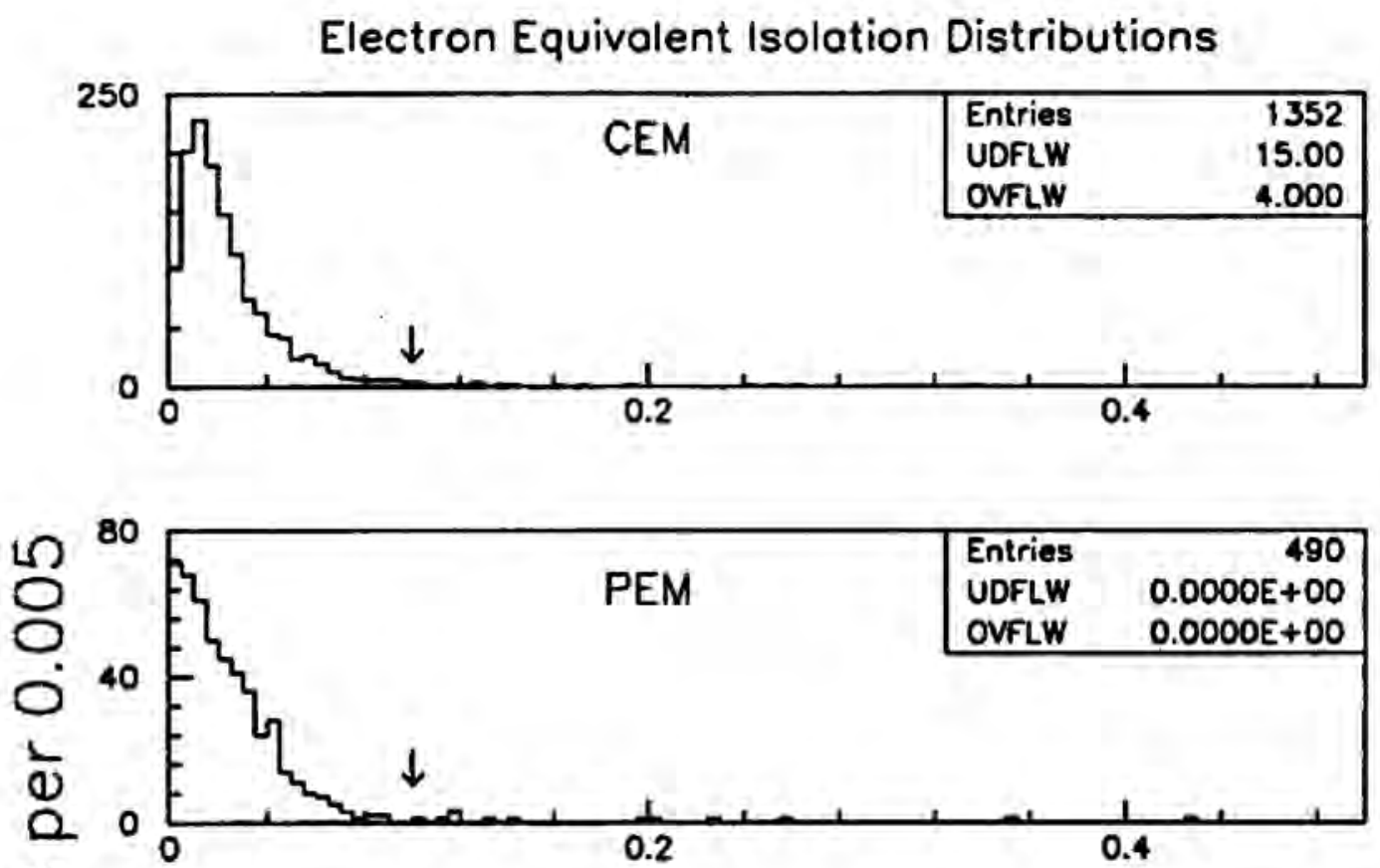

\#

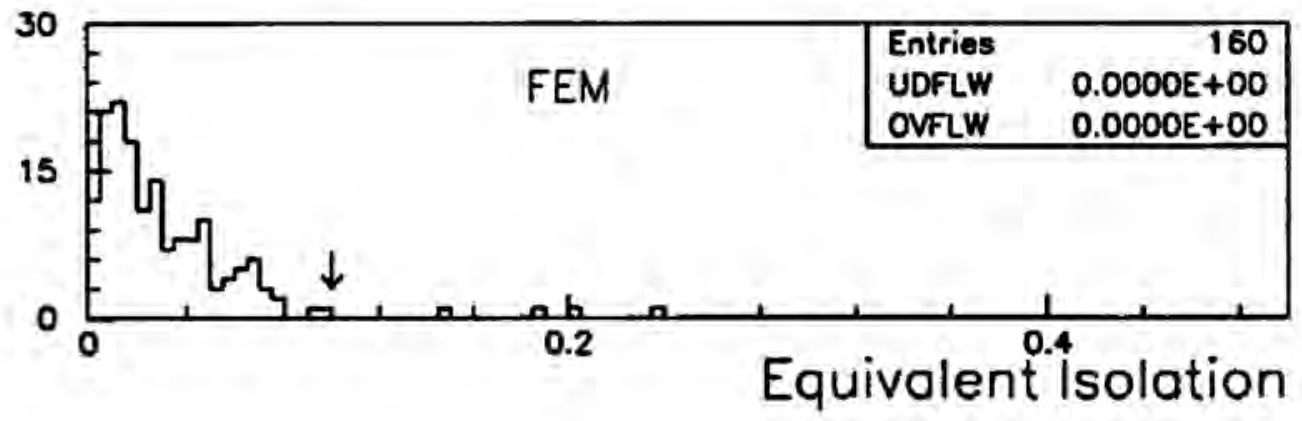

Figure 4.5: The distribution of equivalent electron isolation, calculated using cones at the same $\eta$ but offset in $\phi$ from the electron. 
Table 4.5: The electron isolation cut efficiencies estimated from equivalent isolation distributions.

\begin{tabular}{lc} 
& $\epsilon_{\text {iso }}$ \\
\hline CEM: & $0.985 \pm 0.04$ \\
PEM: & $0.976 \pm 0.04$ \\
FEM: & $0.975 \pm 0.04$ \\
\hline
\end{tabular}

Table 4.6: The total electron selection efficiency.

\begin{tabular}{lc} 
& $\epsilon_{\text {tot }}=\epsilon_{\text {iso }} \times \epsilon_{\text {ele }}$ \\
\hline CEM tight cuts: & $0.855 \pm 0.043$ \\
CEM loose cuts: & $0.920 \pm 0.041$ \\
PEM loose cuts: & $0.924 \pm 0.044$ \\
FEM loose cuts: & $0.975 \pm 0.073$ \\
\hline
\end{tabular}

\subsection{Muon Selection}

Muons are reconstructed from hits in muon drift chambers located behind the central calorimeter, as discussed in Section 3.2.3. This in general is a clean signature because most of the background hadronic particles from jet production are absorbed by the calorimeter in front. However, energetic pions can punch through the calorimeter leading to hits in the muon drift chamber. Also, for $Z \rightarrow \mu \mu$ selection, we allow one of the muons to pass only loose cuts and do not require it to be in regions covered by muon drift chambers. We need a set of cuts on muon quality parameters to make the optimal event selection.

\subsubsection{Muon Fiducial Region}

Fiducial requirements are necessary to ensure muons are detected in regions with uniform efficiencies. The muon drift chambers are expected to be fully efficient near the $\phi$ edges, as the drift field should be uniform in the azimuthal direction. This is verified by the uniformity in $W$ decay muon occupancy as a function of the muon hit $\phi$ position. The muon drift chambers are not expected to be fully efficient near the $z$ edges, however, because of drift field distortion effects near the ends of the chambers. This can be seen in cosmic 


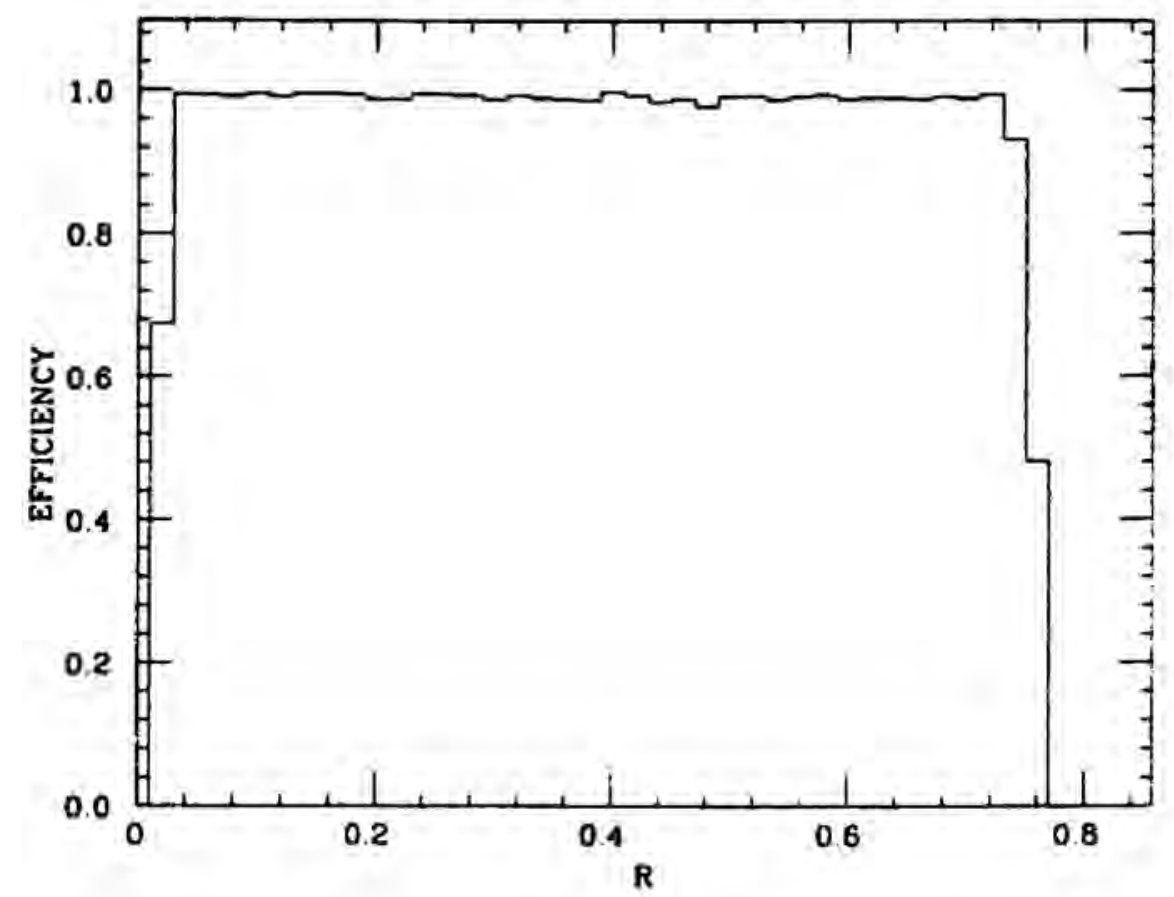

Figure 4.6: The muon detection efficiency as a function of $z$ position. $R$ is the charge division ratio used to determine $z$. A $\mathrm{R}$ value near 1.0 corresponds to the $56^{\circ}$-end of the muon drift chamber.

ray studies, as shown in Figure 4.6. Here, the $z$ position is plotted in terms of the charge division ratio R.[36] The fiducial cuts are listed in Table 4.7. The muon position is given by the drift chamber measurements. There are some events with ADC overflows, and thus no $z$-position measurement. These are probably due to multiple hits. Multiple-hits can occur, for example, when more than one pions from a jet punch through the calorimeter. It can also happen when a knocked-on electron in the back of the calorimeter goes into the drift chamber along with the muon. These events are removed. The resulting inefficiency is found to be less than $2 \%$ using muons from $W$ and $Z$ decays. Also, one chamber, the middle module in wedge 2 on the east side, is removed from the fiducial region because most muons detected in this chamber fail the track matching cuts.

The class of muons detected in muon drift chambers are called CMUOs - Central MUon Objects. For $Z \rightarrow \mu \mu$ selection, a high transverse momentum CTC track satisfying the minimum ionizing criteria can be used as the second muon. These are called CMIOs Central Minimum Ionizing Objects. The fiducial requirement for CMIOs is simply that 
Table 4.7: Fiducial region of the central muon drift chamber. Azimuthal direction is measured in local coordinate with respect to the center of a wedge.

\begin{tabular}{c|c|c|c|c|c}
\hline & \multicolumn{2}{|c|}{ Azimuthal Direction } & \multicolumn{3}{c}{$z$ Direction } \\
\cline { 2 - 6 } & $\phi$ & $x$ & $\theta$ & $\eta$ & $z$ \\
\hline $\begin{array}{c}\text { Muon Chamber } \\
\text { Coverage }\end{array}$ & $1.19-13.81^{\circ}$ & $\pm 38.1 \mathrm{~cm}$ & $88.5-55.9^{\circ}$ & $0.026-0.63$ & $9.1-235 \mathrm{~cm}$ \\
\hline $\begin{array}{c}\text { Fiducial } \\
\text { Requirements }\end{array}$ & $1.5-13.5^{\circ}$ & $\pm 36.6 \mathrm{~cm}$ & $87.7-57.0^{\circ}$ & $0.040-0.61$ & $13.9-226 \mathrm{~cm}$ \\
\hline
\end{tabular}

it passes through all 9 superlayers in the CTC, or equivalently, $|\eta|<1.0$ measured with respect to the center of CDF.

\subsubsection{Muon Identification Cuts}

The muon identification criteria are designed to reject fake muon backgrounds from energetic pion punch-through. An interacting punch-through pion tends to deposit a larger amount of energy in the calorimeter than a muon. The spectra of muon and pion energy deposition in the calorimeter were measured at the test beam, and are shown in Figure 4.7.[37] The low energy peak in the pion energy spectrum contains a small amount of muon contamination in the pion beam, as well as non-interacting pions. But the majority of the pions deposit more energy than muons. In any case, most non-interacting pion punch-through would show up as muons inside a jet, that is, non-isolated muons. As we will see, this does not present a problem for selecting a clean $Z \rightarrow \mu \mu$ sample.

The muon quality cuts for selecting both CMUOs and CMIOs are the following.

- Track quality: the CTC track associated with a muon candidate must be of good quality so that beam-constrained fitting can be done. It also must not be identified as a cosmic ray.

- $z$-vertex matching: the difference in the $z$-vertex from the CTC track and the VTPC must be less than $5 \mathrm{~cm}$. 

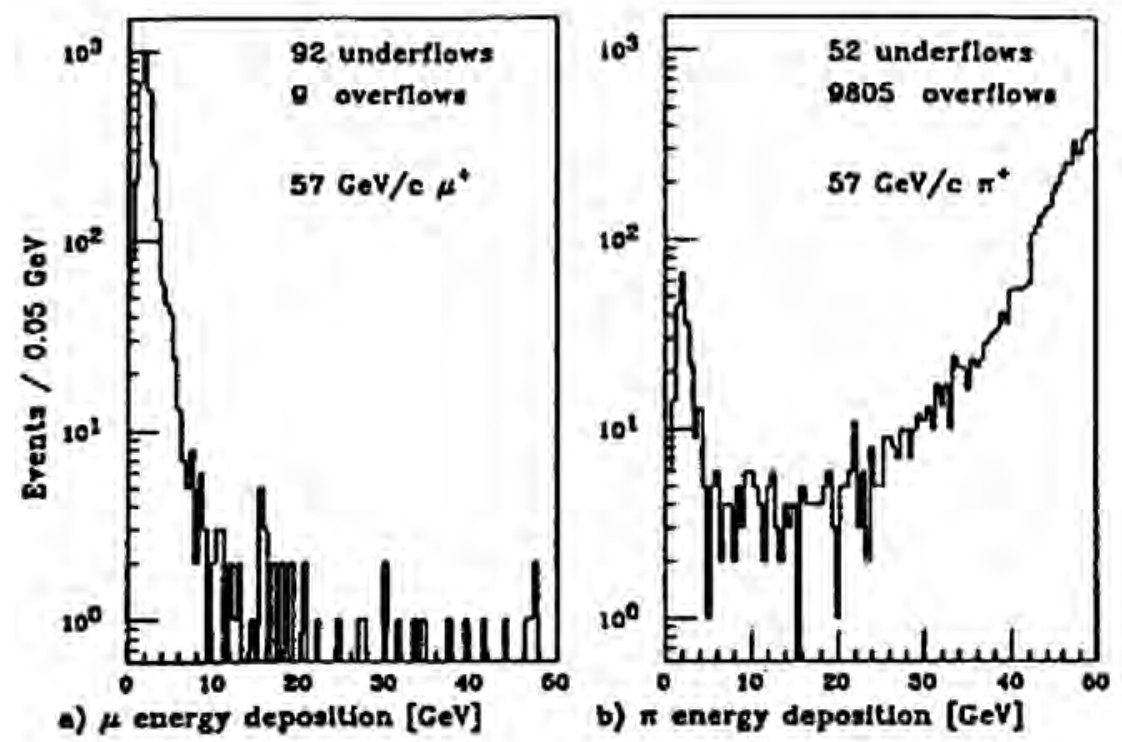

Figure 4.7: The muon and pion energy spectra measured in the central calorimeters at the test beam. The low energy peak in b) contains both non-interacting $\pi$ 's and a $3-4 \% \mu$ contamination in the $\pi$ beam.

- $E_{\text {em }}\left(E_{\text {had }}\right)$ : electromagnetic (hadronic) energy in the calorimeter tower traversed by the muon, as indicated by extrapolating the CTC track through the calorimeter, must be consistent with that for a minimum ionizing particle, and are required to be less than $2 \mathrm{GeV}(6 \mathrm{GeV})$. These cuts are used to reject interacting pions.

For identifying a good CMUO, we further require

- $\Delta x$-matching: the $x$-position measured by the drift chamber must match an extrapolated CTC track to within $2 \mathrm{~cm}$.

The distributions of these cut variables are shown in Figure 4.8 and Figure 4.9. Also shown in Figure 4.9 is the isolation of the muons, defined as for the electrons:

$$
I s o_{\mu}=\frac{\sum_{i} E_{T}^{i}-E_{T}^{\mu}}{p_{T}}
$$

where $E_{T}^{\mu}$ is the energy of the tower traversed by the muon, and $p_{T}$ is the transverse momentum of the muon. It is clear from the plot that the majority of the muons selected 


\section{CMUO Cuts}
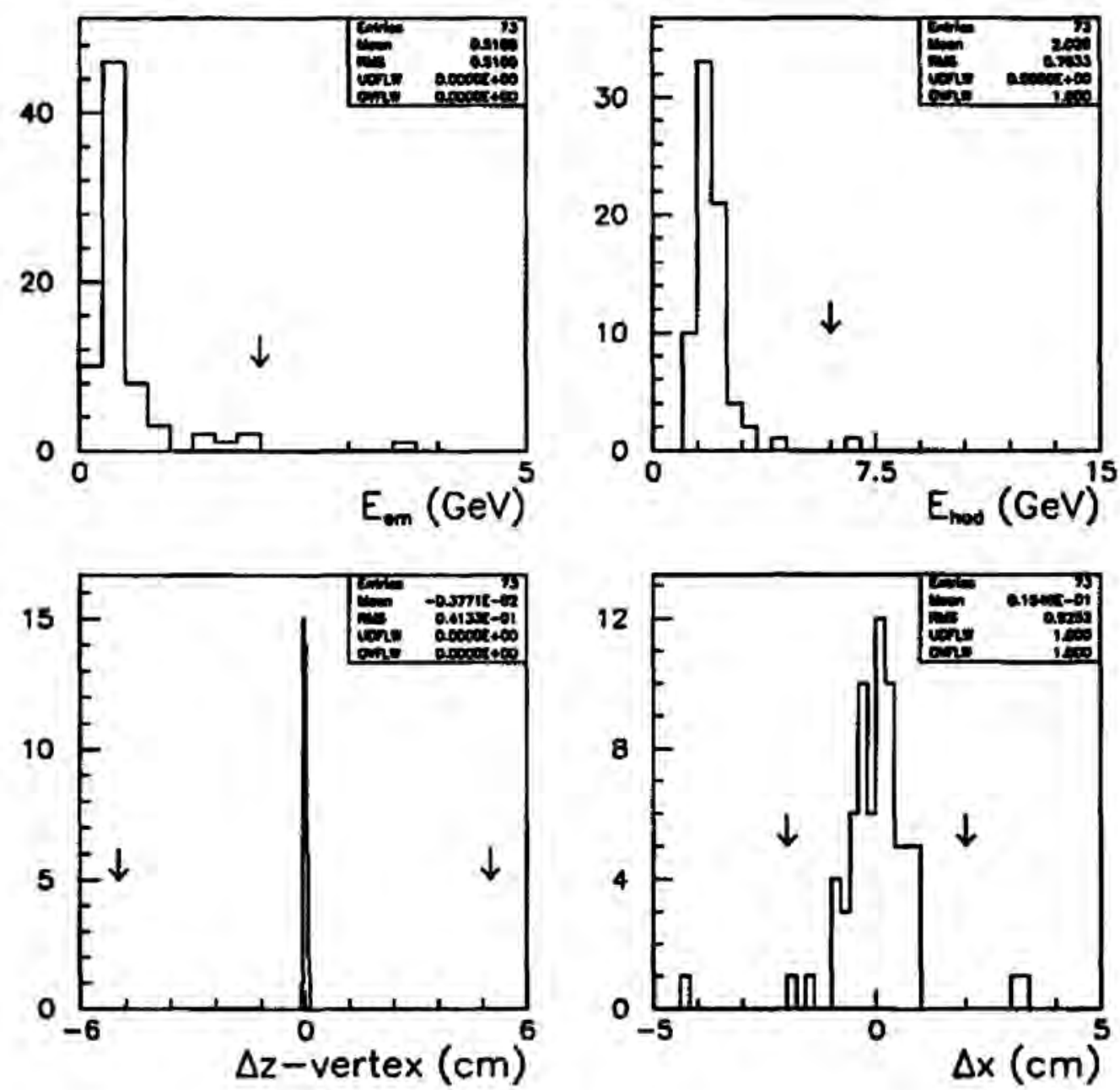

Figure 4.8: The distributions of cut variables used in CMUO muon identification. The plots are made with a sample of $Z$ CMUO muons used in the efficiency measurement. 
with the above cuts are isolated. We do not make any muon isolation cut in this analysis. The plots are made with a sample of $Z$ muons used in efficiency measurement. We describe muon selection efficiency measurement in Section 4.2.3.

\subsubsection{Muon Identification Cut Efficiency}

The muon quality cut parameters are described in Section 4.2.2. The cuts are listed in Table 4.8. The only difference between the tight cut and the loose cut is the requirement of a muon drift chamber track for the tight cut. Also listed in Table 4.8 are the efficiencies of the cuts. The efficiencies of the mnon quality cuts are measured from a sample of muons selected from the $Z$ data sample. This muon sample is selected, as in the electron efficiency measurement, as follows:

1. For each event, require at least one muon passes the tight cuts listed in Table 4.8.

2. The second muon is included in the sample for loose cut efficiency measurement if it

- is in the region $|\eta|<1.0$,

- has at least $10 \mathrm{GeV} / c$ of $p_{T}$, and

- forms a mass between 75 and $105 \mathrm{GeV}$ with the muon selected in step 1 .

3. The second muon is included in the sample for tight cut efficiency measurement if it

- is a CMUO, that is, it has a track in the muon drift chamber,

- has at least $20 \mathrm{GeV} / c$ of $p_{T}$, and

- forms a mass between 75 and $105 \mathrm{GeV}$ with the muon selected in step 1 .

Note that if both muons are CMUOs, both can be included in the sample for tight cut efficiency measurement.

The efficiency of the set of cuts listed in Table 4.8 can then be simply obtained from the number of muons that passes the cuts. The CTC tracking efficiency depends on the number 


\section{CMIO Cuts}
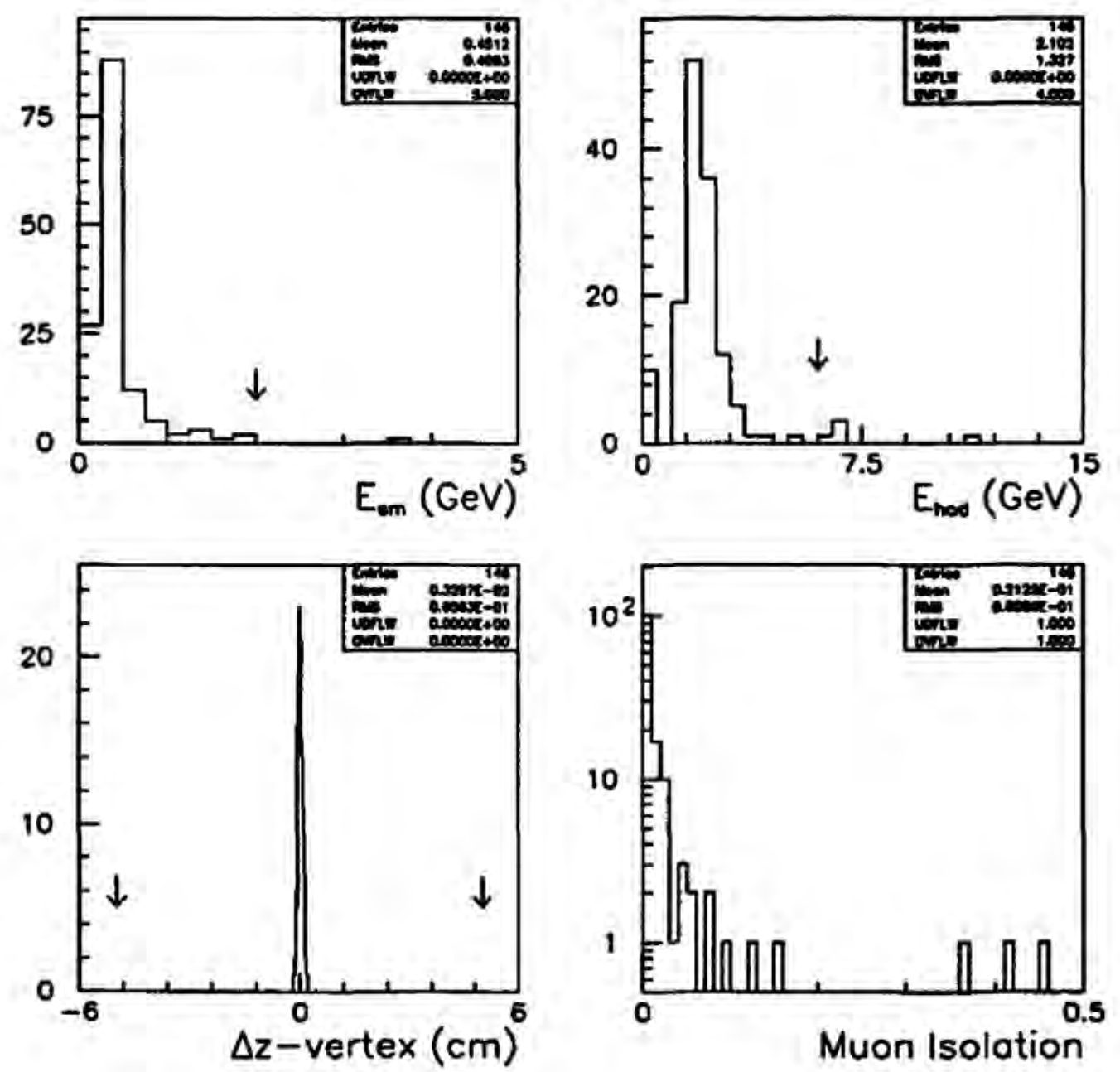

Figure 4.9: The distributions of cut variables used in CMIO muon identification. The plots are made with a sample of $Z$ CMIO muons used in the efficiency measurement. (Note that isolation cut is not used in this analysis). 
Table 4.8: The muon identification cuts and efficiencies.

\begin{tabular}{ccc}
\hline $\begin{array}{c}\text { Muon } \\
\text { Quality }\end{array}$ & $\begin{array}{c}\text { CMUO } \\
\text { Tight Cuts }\end{array}$ & $\begin{array}{c}\text { CMUO/CMIO } \\
\text { Loose Cuts }\end{array}$ \\
\hline Track & Good & Good \\
Quality & CTC Track & CTC Track \\
\hline $\begin{array}{c}z \text {-vertex } \\
\text { Match }\end{array}$ & $\mid z_{\text {track- }}$ - VTPC $\mid<5 \mathrm{~cm}$ & $\mid z_{\text {track }}-2$ VTPC $\mid<5 \mathrm{~cm}$ \\
\hline Track & $|\Delta x|<2.0 \mathrm{~cm}$ & \\
Match & & \\
\hline Min- & $E_{\text {em }}<2 \mathrm{GeV}$ & $E_{\text {em }}<2 \mathrm{GeV}$ \\
Ionizing & $E_{\text {had }}<6 \mathrm{GeV}$ & $E_{\text {had }}<6 \mathrm{GeV}$ \\
\hline \hline Efficiency & $0.943 \pm 0.028$ & $0.927 \pm 0.021$ \\
\hline
\end{tabular}

of superlayers traversed by the track.[38] For this analysis, CTC tracks are required to pass all superlayers, so that the tracking efficiency is uniform for tracks at different $\theta$ 's.

Note that we do not need to make an isolation cut on the muons to get a clean $Z \rightarrow \mu \mu$ sample. We use the fact that the underlying event energy deposition should be identical for both $Z \rightarrow \mu \mu$ and $Z \rightarrow e \epsilon$ events to study the systematic effects in the electron isolation cut efficiency measurement in Section 4.3.1.

\subsection{Systematics in the Lepton Efficiencies}

We now investigate the systematic effects in our lepton efficiency measurement. We discuss the systematic uncertainty in the electron isolation cut efficiency due to the assumption of azimuthal symmetry in the underlying event energy depositions with respect to the electron position. We also discuss possible dependence of the efficiencies on the $Z p_{T}$.

\subsubsection{Isolation Cut Efficiencies: Muons vs. Electrons}

Because the event topology should be identical for $Z \rightarrow e e$ and $Z \rightarrow \mu \mu$ events, we expect the underlying event energy deposition to be the same for the two decay modes. Muon isolation is not used in muon identification. It is studied here in comparison with electron isolation. The isolation variable for muons is defined in Equation 4.7. Efficiency of the muon isolation cut is abtained, as for the electron isolation cut, by using the azimuthal 


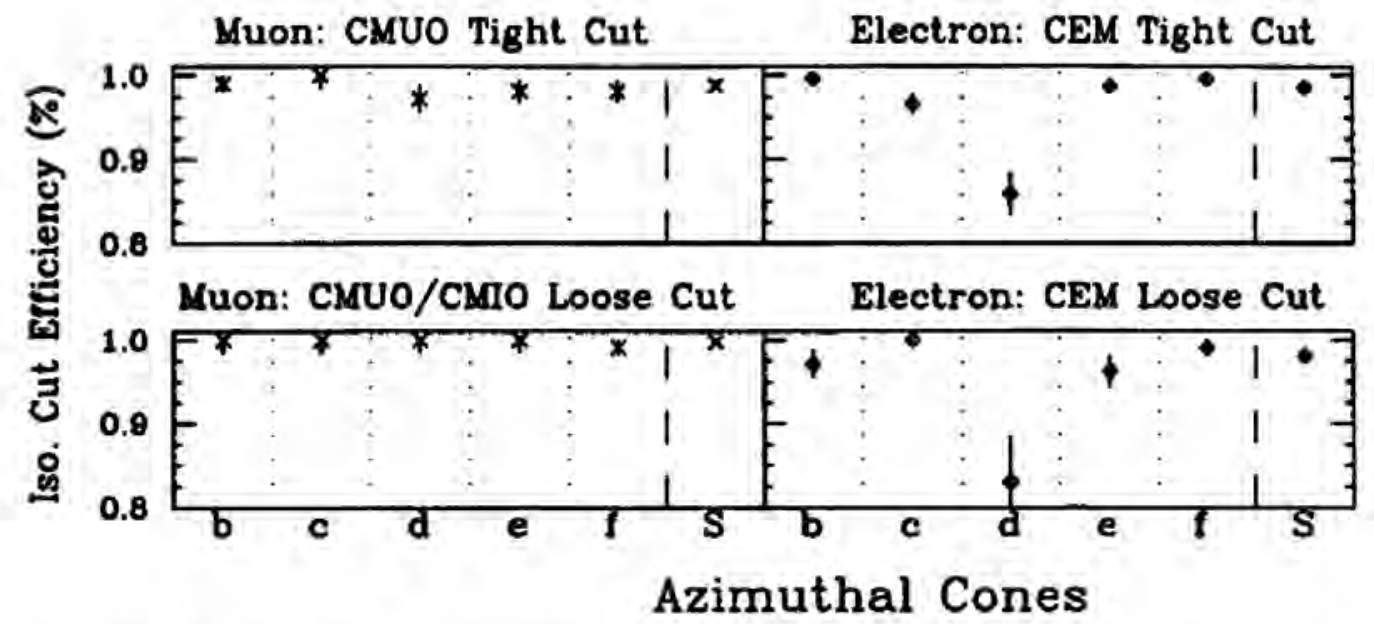

Figure 4.10: The efficiency of isolation cut for each $\phi$-cone for central muons and electrons. Cone " $S$ " is the average of cones " $b$ ". "c", "e", and " $p$ ".

symmetry in the underlying event energy deposition, and calculating equivalent isolations as if the muon were in a different $\phi$ position. The isolation efficiency obtained for each $\phi$-cone is shown in Figure 4.10 for both muons and CEM electrons. The $\phi$-cone " $\mathrm{d}^{n}$ is $180^{\circ}$ away from the lepton being studied and picks up energy deposited in the calorimeter by the other lepton in the event. We ignore this $\phi$-cone in our efficiency calculations. For muons, the variation among the other four cones is $1 \%$. For CEM electrons, $\mathrm{jt}$ is $4 \%$ and is assigned as a systematic uncertainty in the electron isolation cut efficiency. This is also true for PEM and FEM electrons. Effects that can lead to larger variation for electrons are fluctuation in EM shower and bremsstrahlung.

\subsubsection{Lepton Efficiencies for Two $Z p_{T}$ Regions}

Ideally, we would measure the efficiency as a function of the $Z p_{T}$. But we are limited by the statistics of the $Z$ data sample. We can still get an estimate of possible $p_{T}$ dependence, by dividing the sample into two $p_{T}$ ranges, above and below $15 \mathrm{GeV} / c$. As shown in Figure 4.11 and Figure 4.12, the efficiencies obtained for the two $p_{T}$ ranges are the same within the statistical uncertainties. 


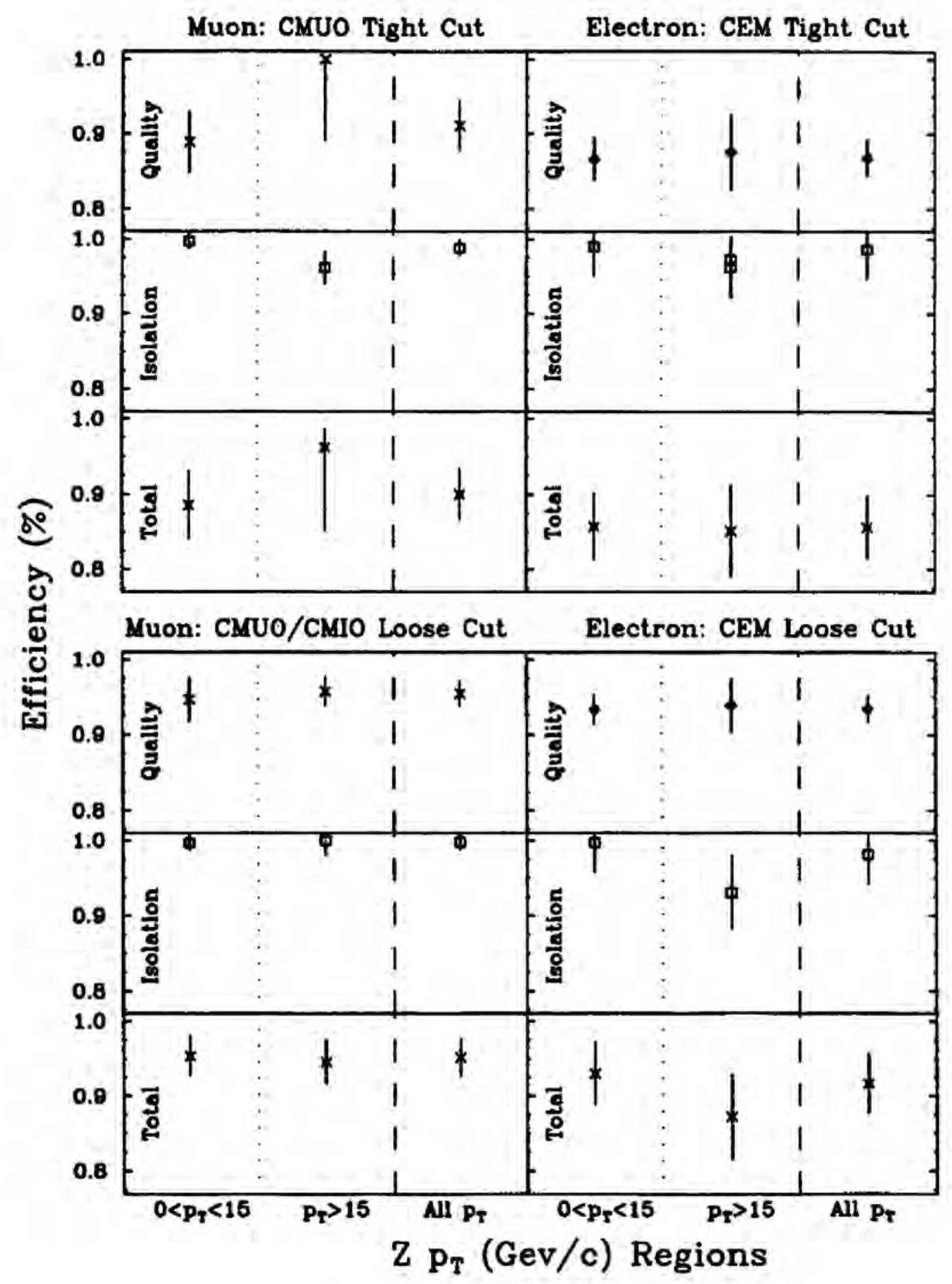

Figure 4.11: The efficiencies for two $Z p_{T}$ regions for central muons compared with central electrons. (Isolation cuts not used in the muon analysis, but presented here for comparison with the electron analysis.) 


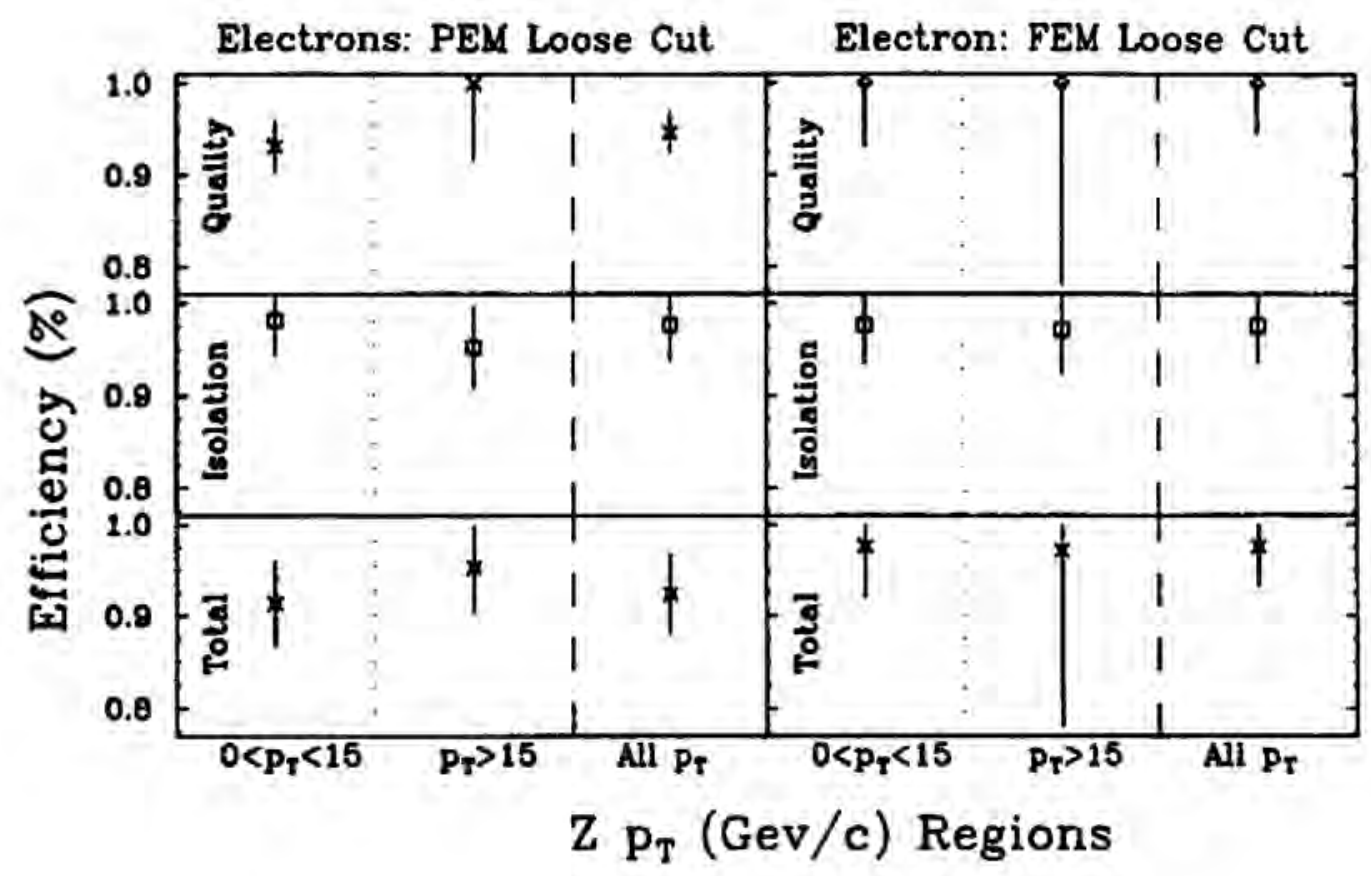

Figure 4.12: The efficiencies for two $Z p_{T}$ regions for FEM and PEM electrons.

\subsection{The Trigger Requirement}

Events in the electron data sample must satisfy the Level 2 central electron trigger, which requires an EM cluster that has at least $12 \mathrm{GeV}$ of $E_{T}$, a ratio of less than 0.125 for the hadronic to electromagnetic energy, and an associated track with $p_{T}>6 \mathrm{GeV} / c$ from the online Central Fast Track (CFT) [19] processor.

Events in the muon data sample must satisfy the central muon trigger. The Level 1 muon trigger requires the presence of a muon track segment above a $p_{T}$ threshold. This corresponds to a cut on the muon track angle. The Level 2 muon trigger requires a track from the CFT to match a muon track segment in $\phi$, and thus implicitly pre-requires a Level 1 muon trigger. The Level 3 muon trigger is essentially the same as the Level 2 trigger, with improved tracking. The track $p_{T}$ threshold is $9 \mathrm{GeV} / c$. 


\subsubsection{Electron Trigger Efficiency}

The electron trigger efficiency is measured using $W$ events in which the electron passes our selection cuts. The $W$ events are selected with the following cuts, which are similar to those used to study the $W$ electron identification efficiency:

- Require a central electron passing our tight cuts.

- Cut on the missing transverse momentum: $F_{T}>20 \mathrm{GeV}$.

- Cut on the significance of the $Z_{T}$ measurement:

$$
\sigma\left(\psi_{T}\right) \equiv \frac{\psi_{T}}{\sqrt{\sum_{\text {towers }} E_{T}}}>2.5 .
$$

- Cut on the $W$ transverse mass:

$$
M_{T} \equiv \sqrt{2 E_{T} \not_{T}\left(1-\cos \left(\phi(e)-\phi\left(H_{T}\right)\right)\right)}>50 \mathrm{GeV} .
$$

- Cut on di-jet events: we reject events with a jet cluster with $E_{T}>10 \mathrm{GeV}$ within $30^{\circ}$ in $\phi$ opposite of the electron candidate.

The trigger efficiency is given by

$$
\epsilon=\frac{N(\text { Satisfied CEM cuts and Trigger })}{N(\text { Satisfied CEM cuts })},
$$

and is measured to be $(97.2 \pm 0.4) \%$.[32] This is the trigger efficiency for C-P and C-F events. C-C events have two chances of satisfying the central electron triggers. The trigger efficiency for C-C events is $(99.9 \pm 0.1) \%$.

\subsubsection{Muon Trigger Efficiency}

In previous studies, the Level 1 muon trigger efficiency has been obtained with a sample of muons from dedicated cosmic-ray runs. It reaches a plateau at $p_{T}>15 \mathrm{GeV} / \mathrm{c}$ at $92.3 \%$. The Level 2 muon trigger efficiency has been measured using a sample of isolated CMUOs from events passing the Level 1 muon trigger and a Level 2 trigger independent of the muon 
trigger such as the Jet, Photon, Electron, or Missing $E_{T}$ triggers. It is determined to be $97.2 \%$ for $p_{T}>15 \mathrm{GeV} / \mathrm{c}$. The timing of cosmic-ray events is different from $\tilde{p} p$ events so that the online track processor behaves differently, and cosmic-ray events cannot be used to find the Level 2 muon trigger efficiency. The combined Level 1 and Level 2 muon trigger efficiency from this series of studies is $(90 \pm 2) \%$.[39]

However, as in the $Z \rightarrow e e$ analysis, we want the muon trigger efficiency for $Z \rightarrow \mu \mu$ events where the muons pass our selection cuts. In the electron analysis, there is considerable overlap between the electron trigger and other triggers such as the Missing $E_{T}$ and the photon triggers. The electron trigger efficiency can be measured from a sample of $W \rightarrow e v$ events that are unbiased with respect to the electron trigger. But because the muon deposits only a small amount of energy in the calorimeter, the $W \rightarrow \mu \nu$ events would usually pass only the muon trigger. That is, the $W \rightarrow \mu \nu$ events are not unbiased with respect to the muon trigger, and we cannot use the $W \rightarrow \mu \nu$ sample to measure the muon trigger efficiency.

Instead, we use the second muon (a CMUO) of the $Z \rightarrow \mu \mu$ events passing the same cuts used in this measurement to measure the muon trigger efficiency. Since there are two muons (CMUOs) in the event and we require only one to satisfy the single muon trigger, the other one is unbiased with respect to the muon trigger. The method is simple: for events obtained with a single muon trigger, if one muon triggered, check if the other muon fired the trigger also; for events obtained with a non-muon trigger, check if both muons fired the muon trigger. This is explained in more detail as follows. Let's call one of the muons "muon-1", the other "muon-2", and

- M1 = muon-1 passed selection cuts,

- M2 = muon-2 passed selection cuts,

- $\mathrm{T} 1=$ muon-1 fired muon trigger,

- $\mathrm{T} 2=$ muon-2 fired muon trigger, 
Table 4.9: The muon trigger efficiencies obtained from $Z$ muons.

\begin{tabular}{cc} 
& $\epsilon$ \\
\hline Level 1: & $0.969 \pm 0.022$ \\
Level 2: & $0.967 \pm 0.023$ \\
Level 3: & 1.0 \\
\hline Total: & $0.937 \pm 0.031$ \\
\hline
\end{tabular}

- $\mathrm{T} 3=\mathrm{T} 1$, or $\mathrm{T} 2$, or any other trigger that enabled this event to be written to tape.

We now have two independent measure of the muon trigger efficiency ( $a$ "." means a logical AND):

$$
\begin{aligned}
& \epsilon(\mathrm{T} 1)=\frac{\mathrm{N}(\mathrm{O} 1 \cdot \mathrm{T} 1 \cdot \mathrm{T} 3)}{\mathrm{N}(\mathrm{O} 1 \cdot \mathrm{T} 3)}, \\
& \epsilon(\mathrm{T} 2)=\frac{\mathrm{N}(\mathrm{O} 2 \cdot \mathrm{T} 2 \cdot \mathrm{T} 3)}{\mathrm{N}(\mathrm{O} 2 \cdot \mathrm{T} 3)} .
\end{aligned}
$$

For $Z \rightarrow \mu \mu$ events, T3 is most likely the single-muon trigger. The only requirement is that T3 not be T1 when muon-1 is being tested (Equation 4.11), or T2 when muon-2 is being tested (Equation 4.12) in the muon trigger efficiency measurement. We combine the measurements from the two muons and the maximum likelihood result is

$$
\epsilon_{\text {CMU Trigger }}=\frac{\mathrm{N}(01 \cdot \mathrm{T} 1 \cdot \mathrm{T} 3)+\mathrm{N}(\mathrm{O} 2 \cdot \mathrm{T} 2 \cdot \mathrm{T} 3)}{\mathrm{N}(\mathrm{O} 1 \cdot \mathrm{T} 3)+\mathrm{N}(\mathrm{O} 2 \cdot \mathrm{T} 3)}
$$

We obtain $\epsilon_{\text {Level1 }}=0.969 \pm 0.022$, and $\epsilon_{\text {Level2 }}=0.967 \pm 0.023$, for a combined $\epsilon_{\text {CMu Trigge }}=$ $0.937 \pm 0.031$. Within the uncertainties, these are consistent with the results of Reference [39]. The muon trigger efficiencies are summarized in Table 4.9. For $Z$ events with two CMUOs, each event has two chances to pass a muon trigger and the efficiency is $(99 \pm 1) \%$.

\subsection{The Selection of $Z \rightarrow e e$ and $Z \rightarrow \mu \mu$ Events}

Using the electron and muon identification criteria described above, we select a clean sample of $Z \rightarrow \epsilon e$ and $Z \rightarrow \mu \mu$ events. The exact identification cuts are listed in Table 4.2 and Table 4.3 for electrons, and Table 4.8 for muons. The event vertex must be within $60 \mathrm{~cm}$ of the center of CDF. In summary, the $Z \rightarrow e \epsilon$ sample is selected by requiring that: 
1. each electron must be inside the fiducial region;

2. at least one central electron must pass the tight cut and have $E_{T}>20 \mathrm{GeV}$;

3. a second electron in the central, plug, or forward regions must pass loose cuts and have $E_{T}>10 \mathrm{GeV}$;

4. the invariant mass must be in the range $75 \mathrm{GeV}<M_{e \epsilon}<105 \mathrm{GeV}$; and

5. the event must pass the Central Electron trigger.

The $Z \rightarrow \mu \mu$ sample is selected by requiring that:

1. each muon must be inside the fiducial region;

2. at least one muon detected in the muon drift chambers must pass the tight cuts and have $p_{T}>20 \mathrm{GeV} / c$;

3. a second muon with $|\eta|<1.0$ (either a CMUO or a CMIO) must pass the loose cuts and have $p_{T}>10 \mathrm{GeV} / c$;

4. the invariant mass must be in the range $75 \mathrm{GeV}<M_{\mu \mu}<105 \mathrm{GeV}$; and

5. the event must pass the Central Muon trigger.

The mass distribution of the $Z$ sample is shown in Figure 4.13, where we included events outside the $Z$ mass window cut. It shows a clear $Z$ mass peak. The number of events outside the $Z$ mass peak is consistent with the Drell-Yan continuum contribution predicted by a Monte Carlo simulation discussed in Section 6.2. The observed $p_{T}$ distribution of these $Z$ candidate events is shown in Figure 4.14. The efficiency of the kinematic and fiducial cuts depend on the $p_{T}$. The $Z$ selection acceptance as a function of the $Z p_{T}$ is obtained from Monte Carlo simulation as discussed in Section 6.2. 


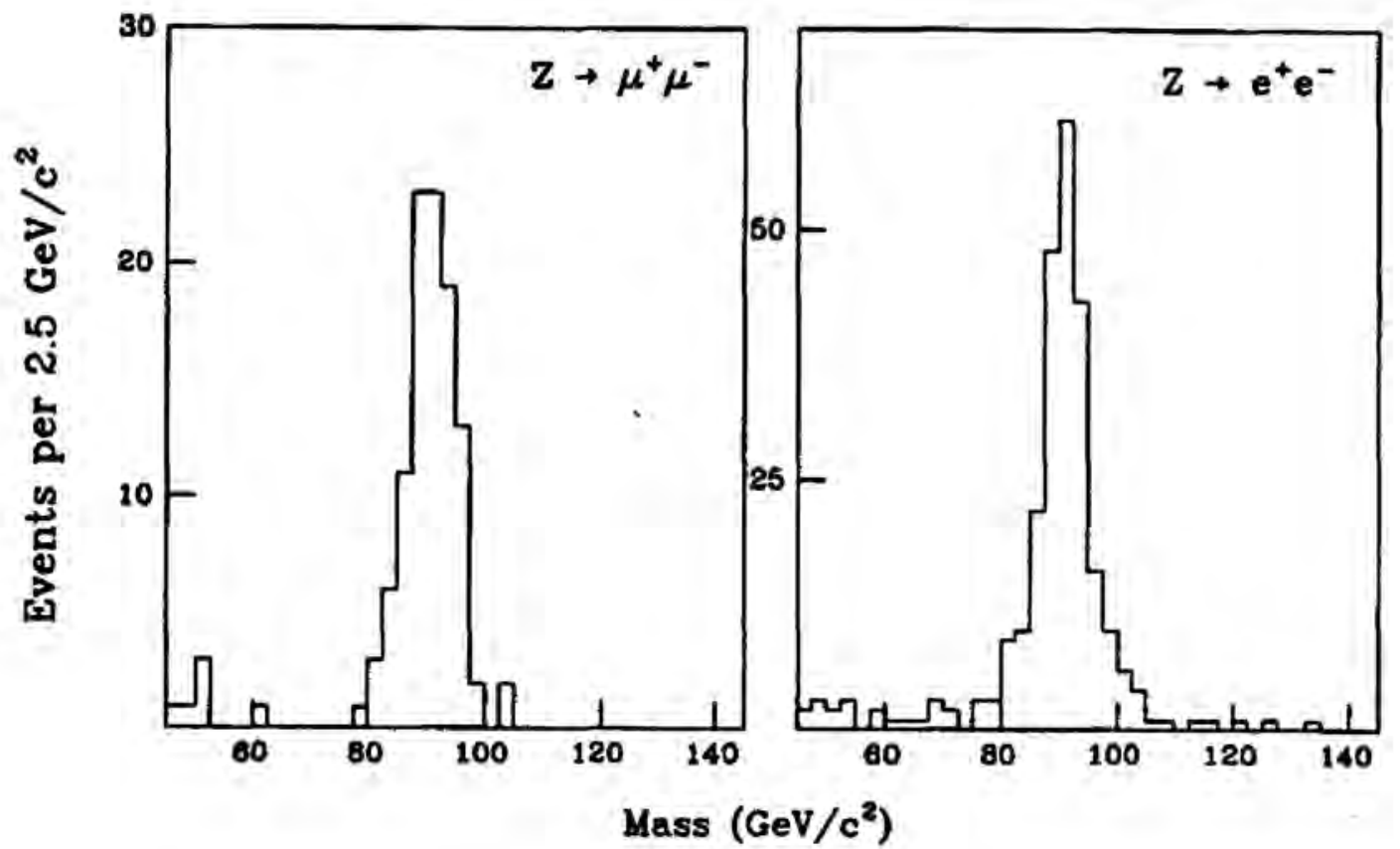

Figure 4.13: The mass distributions of $\mu \mu$ and $e e$ pairs, including low mass pairs. There are $103 Z \rightarrow \mu \mu, 235 Z \rightarrow e \epsilon$ candidate events with invariant mass between 75 and $105 \mathrm{GeV}$.

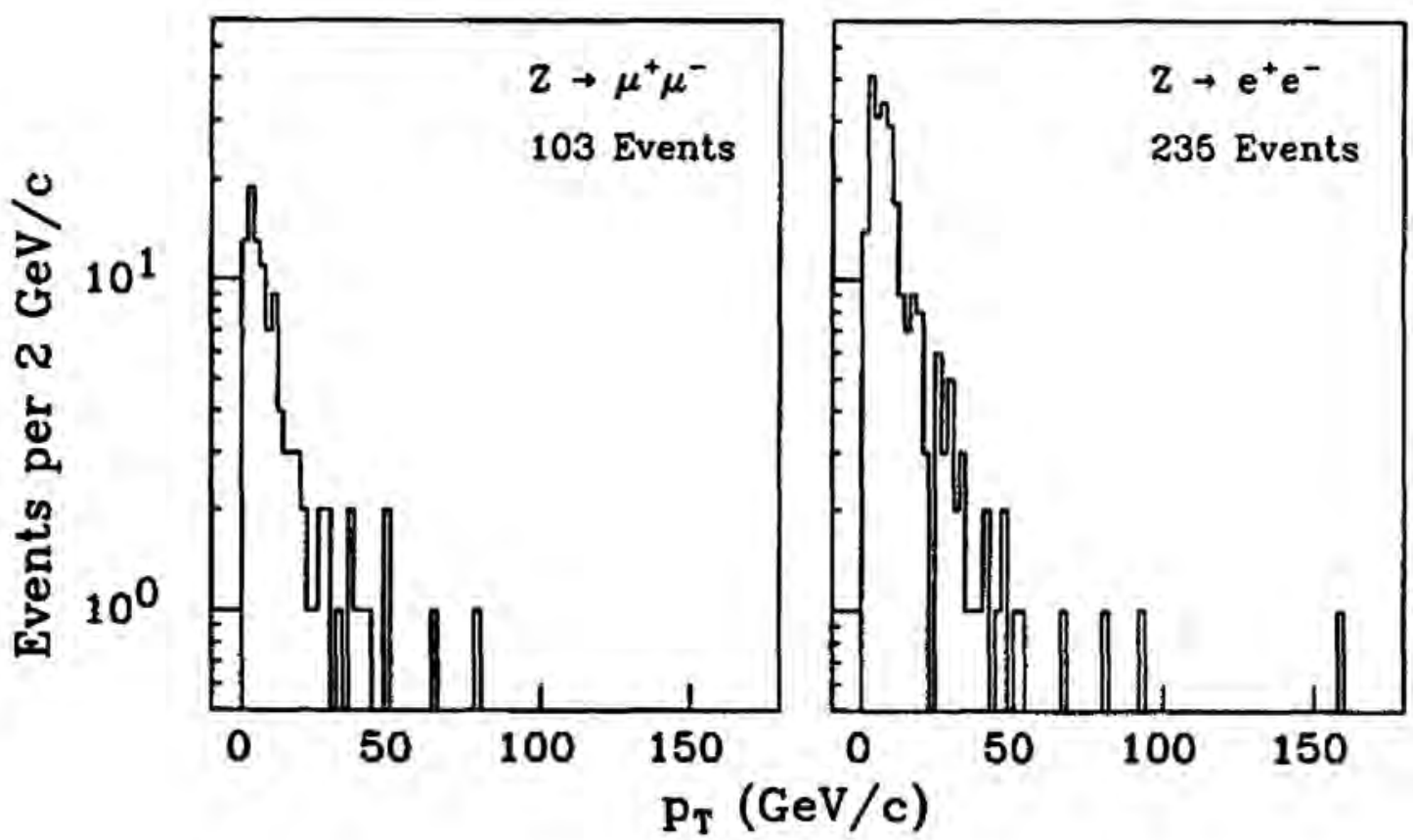

Figure 4.14: The observed $p_{T}$ distributions of the $Z \rightarrow e e$ and $Z \rightarrow \mu \mu$ candidates. 


\section{Chapter 5}

\section{Event Properties of the $Z$ Data Sample}

Thus far in our analysis, we have made selection only on the $Z$ decay leptons. It is worthwhile now to examine the rest of the event in our $Z$ sample, before proceeding to measure $d \sigma / d p_{T}$ and confront it with QCD predictions. In terms of QCD, we expect jets to be produced along with the $Z$ gauge bosons. It is also interesting to see if there is any deviation from QCD expectations, such as a large missing transverse energy in the event.

\subsection{The Jets in $Z$ Events}

In the framework of QCD, the transverse momentum of the $Z$ results from the production of quarks or gluons along with the $Z$. The quarks or gluons are associated with the jet clusters experimentally observed. Jet clustering is discussed in Section 3.2.4. For this analysis, a jet is selected with the following cuts:

- A jet cluster with at least $10 \mathrm{GeV}$ of observed transverse energy. (These are "uncorrected $^{n}$ jet energies.)

- The pseudo-rapidity of the jet cluster centroid, $\eta_{j e t}$, with respect to the center of the detector, must satisfy $\left|\eta_{j e t}\right|<3.5$.

We examine the $Z p_{T}$ for events with and without jets. Events without any jets are called 0 -jet events, those with one and only one are called 1-jet events, and those with at least two 
jets are called 2-jet events. (There are two 3-jet and one 4-jet events in this sample. These events are described in more detail in Section 5.6.1.) The $p_{T}$ distributions are shown in Figure 5.1 for both the $Z \rightarrow e e$ and $Z \rightarrow \mu \mu$ samples, Clearly, the $p_{T}$ distribution for 0 -jet events is peaked towards the low end, whereas it is broader for the 1 -jet events. This is what we expect, at least qualitatively. That is, when the boson is produced recoiling against an energetic jet (the quark or gluon), it would tend to have a large transverse momentum. Also note the similarity between the $Z \rightarrow e e$ and $Z \rightarrow \mu \mu$ events in this regard. For 1 -jet events, the distributions of the jet $E_{T}, \eta$, and EM energy fraction are shown in Figure 5.2. The same distributions for the second most energetic jet in 2-jet events are shown in Figure 5.3.

\subsection{The Missing $E_{T}$ in $Z$ Events}

The missing transverse energy measured by the calorimeter is defined as:

$$
\overrightarrow{H_{T}} \text { cal }=-\sum_{i} E_{T}^{i} \cdot \hat{n}_{i},
$$

a vector sum of the transverse energies in calorimeter towers in the region $|\eta|<3.6$. This gives the missing $E_{T}$ in $Z \rightarrow \epsilon \epsilon$ events. In $Z \rightarrow \mu \mu$ events, the muons only deposit a minimum ionizing amount of energy in the calorimeter. Each component of the $\vec{H}_{T_{\text {cal }}}$ is corrected using the muon momentum measured by the CTC:

$$
\vec{H}_{T_{\mu}}=\vec{H}_{T_{\text {cal }}}+\vec{E}_{T_{\text {cal }}^{\mu}}^{\mu}-{\overrightarrow{p_{T}}}^{\mu}
$$

where $E_{T_{\mathrm{cal}}}^{\mu}$ is the muon energy deposition in the calorimeter tower traversed by the muon.

The distributions of $E_{T}$ are shown in Figure 5.4. Note that the distribution is peaked towards the lower end for $Z+0$-jet events. But it is broader for $Z+1,2$-jet events, probably due to jet energy measurement error. In fact, as discussed in Section 5.6.2, the very large $F_{T}$ 's in the three $Z \rightarrow e e$ 1-jet events are due to jet energy mis-measurements. Also note that the $F_{T}$ distributions are narrower in the $Z \rightarrow \mu \mu$ events, as can be seen in the 0 jet events. This is probably due to the fact that the electron showers in the calorimeter, whereas the muon is minimum ionizing, and a measurement error in the electron energy would lead to missing $E_{T}$. 


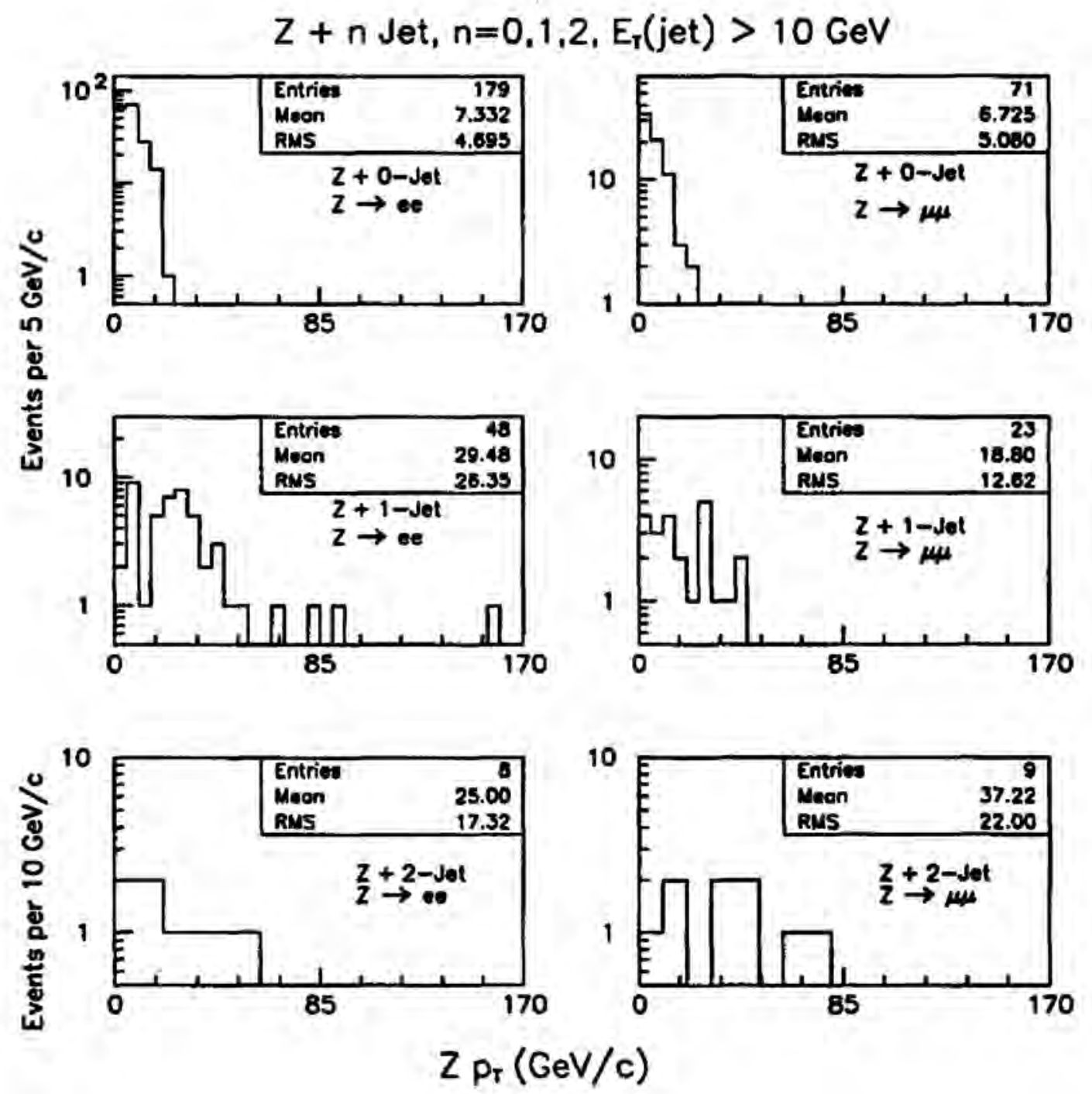

Figure 5.1: The $Z p_{T}$ distributions for events with and without jets. 

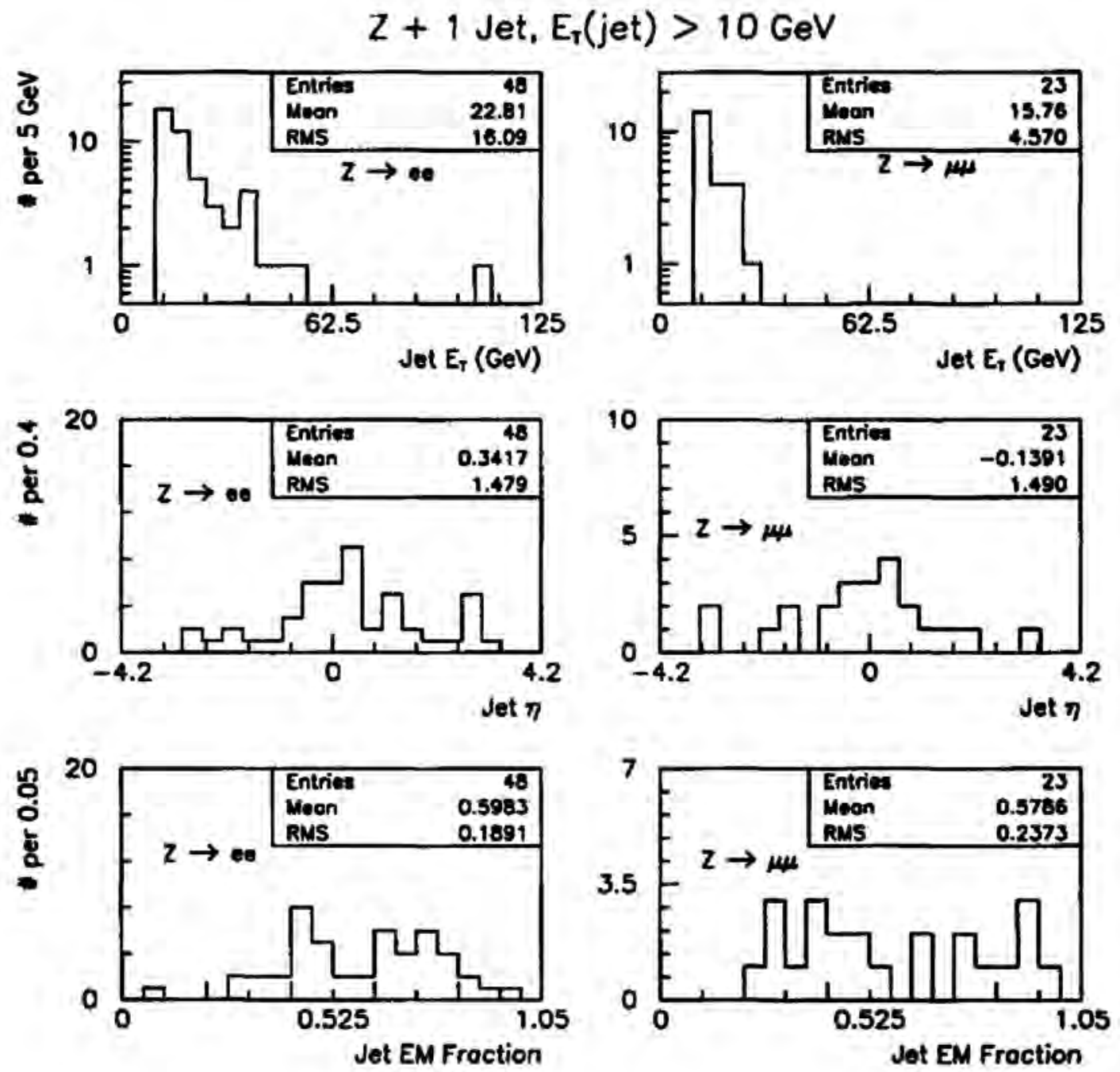

Figure 5.2: The distributions of some jet variables for $Z+1$-jet events. 

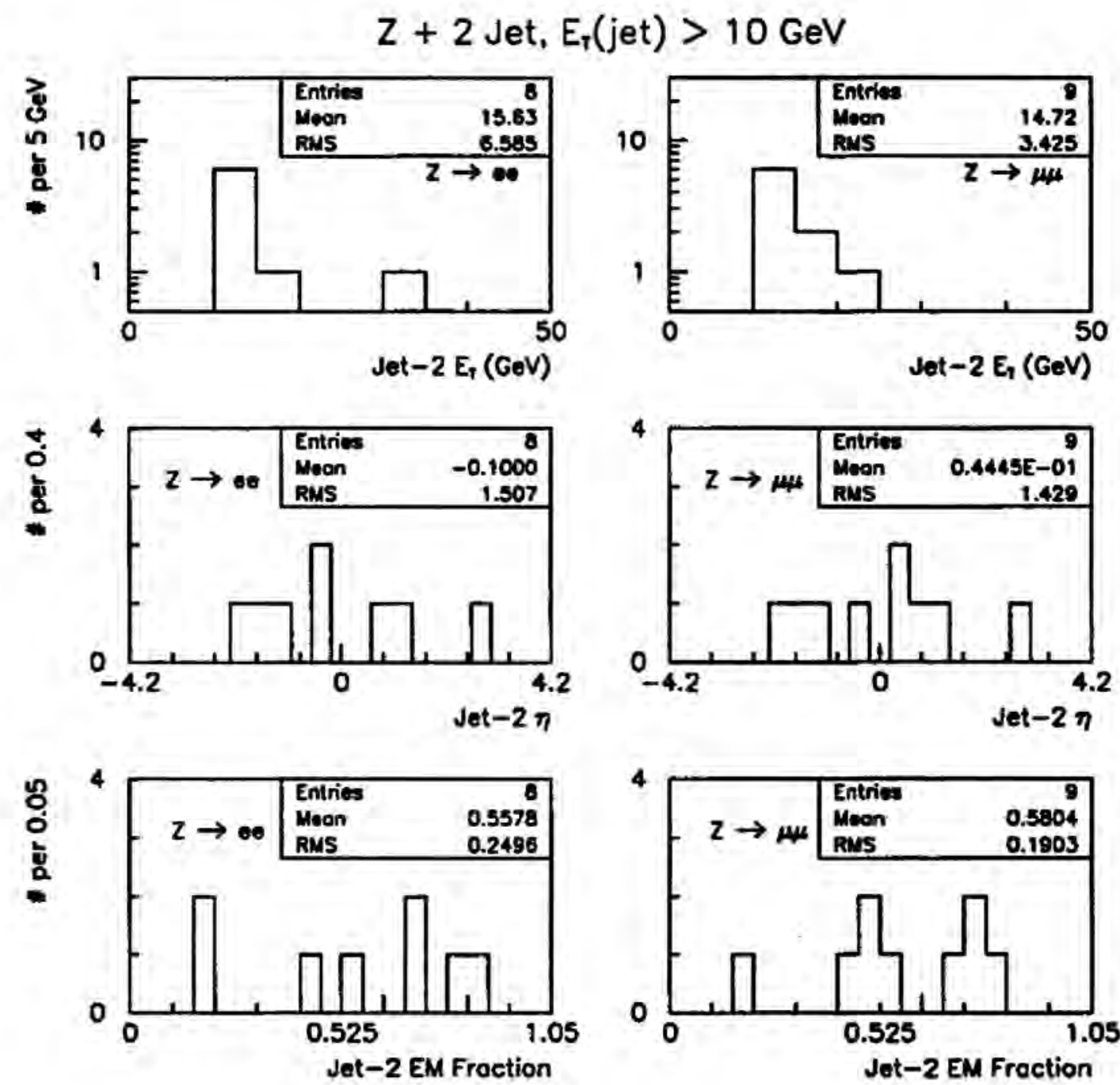

Figure 5.3: The distributions of some jet variables for the second jet in $Z+2$-jet events. 

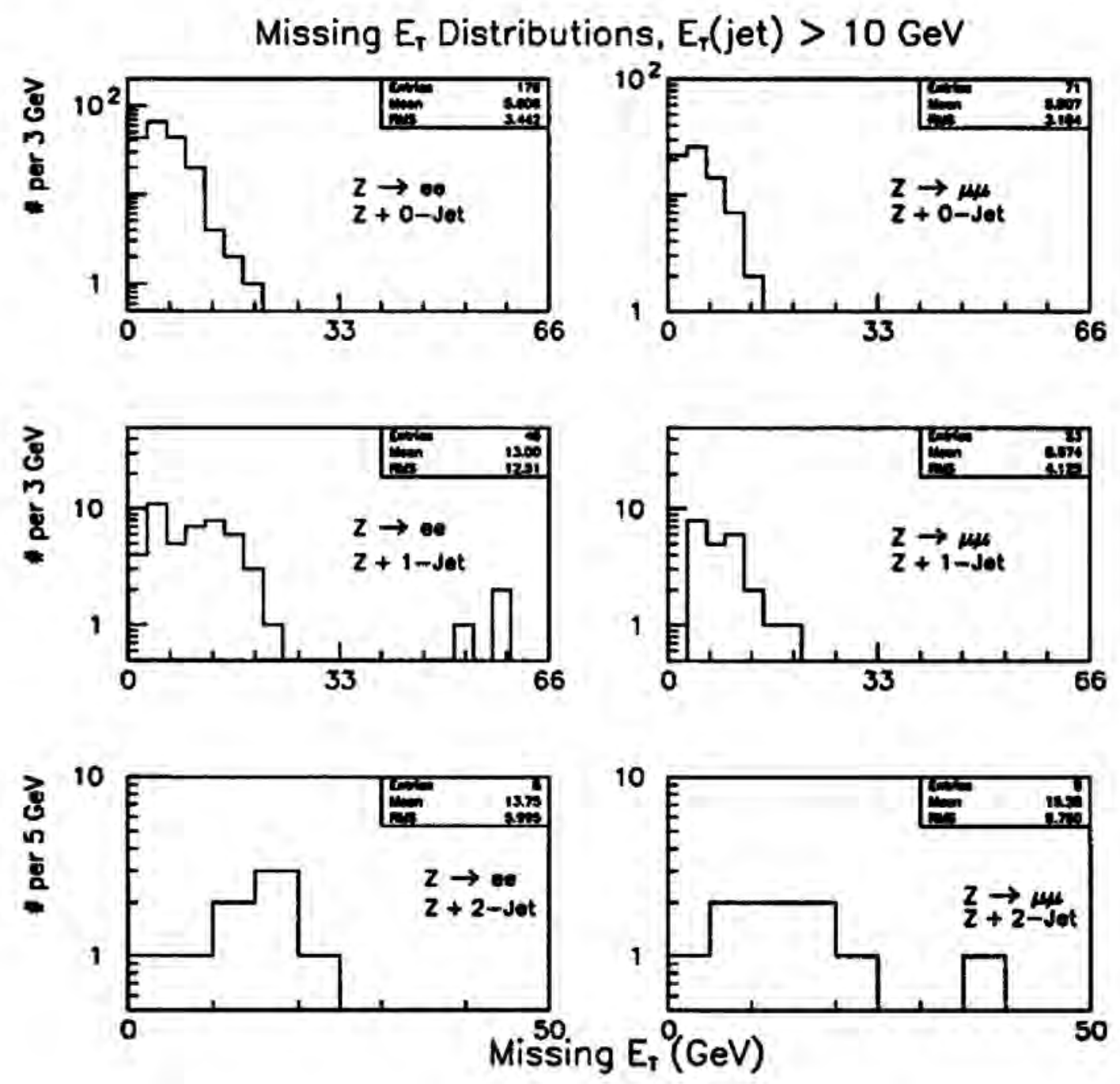

Figure 5.4: The Missing $E_{T}$ distributions in $Z$ events. 


\subsection{Theoretical Expectations}

In order to make theoretical predictions that can be compared directly with experimental observations, we must solve two basic problems. First, for QCD calculations involving massless quarks and gluons, cuts must be made at the parton level to remove singularities. Second, the jet clusters observed in our experiment consist of hadrons, whereas QCD matrix element calculations are done at the parton level. These problems can be solved using a Monte Carlo simulation. The Monte Carlo uses QCD matrix elements to calculate cross sections. A fragmentation model and a detector model tuned to test beam and di-jet data are used to transform the partons into jet clusters. The detector model also accounts for detector energy resolution and acceptance of the event selection cuts. The Monte Carlo predictions thus account for the various experimental effects. In Section 5.6, we compare these predictions directly with observed (uncorrected) data.

\subsubsection{The Papageno Monte Carlo Event Generator}

The Papageno [40] Monte Carlo generates $\ddot{p} p$ collision events using first order QCD matrix elements. For each specified physical process, the final states must first be defined, with cuts to avoid singularities in the matrix elements. For example, in $\bar{p} p \rightarrow Z+1$-jet, we require the parton produced along with the $Z$ to have at least $5 \mathrm{GeV}$ of transverse momentum. The kinematics of the event are chosen randomly in the phase space of n-body topologies, along with a longitudinal boost based on a structure function parametrization. If the kinematics of the event are within the specified region, the matrix element is calculated. As output, particles are produced at the parton level. Each event is given a weight, describing the probability for that event. The sum of the event weights is the total cross section for the specified process.

\section{5,3.2 The Parton Fragmentation Simulation}

To simulate real events, we fragment the partons into particles. Fragmentation determines the number and the energy spectra of these particles. The jet fragmentation model is similar 
to the one used in the ISAJET [41] Monte Carlo. The parton longitudinal momentum is distributed among the fragment hadrons according to the independent fragmentation scheme of Field and Feynman [42]. The hadrons are also given transverse momenta with respect to the jet axis. After fragmentation, the jets acquire mass, even though the original parton is massless. For generated boson + jet events, the hadron momenta are rescaled so that the jet 3 -momentum is the same as the original parton momentum, and thus the boson momentum is unchanged in the fragmentation process.' The underlying event is also simulated. A theoretical model is used, as in the ISAJET Monte Carlo.

The fragmentation model is originally tuned on CDF di-jet data, using the energy and multiplicity distributions of charged tracks inside the jet. For vector boson studies, the fragmentation is re-tuned on CDF $W+$ jets data, using the same procedure. In the same process, the underlying event contribution is also tuned, using the energy and multiplicity distributions of charged tracks inside a cone, of the same size as the jet clustering cone $(R=0.7), 90^{\circ}$ away from the jet in $\phi .[45]$

\subsubsection{The Detector Simulation}

The QFL [43] simulation is used to model the detector response to jet particles. QFL models the CDF calorimeter tower geometry, including the cracks, and its response to particles. To speed up the simulation, the particles are not stepped through the calorimeter at omall intervals. Instead, the energy deposited by a particle traveling through the calorimeter is determined by the total amount of material traversed. The average response is given by a set of "scale" factors determined by simulating test beam measurements, that is, by putting Monte Carlo pions and electrons at various energies into the calorimeter tower center and matching the simulated detector response to test beam data. The actual detector response linearity is studied using real test beam pions (electrons) in the energy range 7 to 227 (5150) $\mathrm{GeV}$ in the central calorimeter, and minimum bias data below $10 \mathrm{GeV}$.[44] The QFL

\footnotetext{
${ }^{1}$ When only jets are generated, the momenta of the resulting hadrons are rescaled so that the total energy of the event is conserved in the fragmentation process.
} 
parameters are tuned to reproduce the observed response behavior.

A simple simulation is used to model the detection and selection of $Z$ decay leptons. (See Section 6.1.2 for details.) The simulation models the lepton fiducial regions, momentum resolutions, and selection efficiencies. The efficiencies are measured from data using $Z$ decay leptons, as discussed in Sections 4.1.3 and 4.2.3. This simulation reproduces well the data,

\subsection{Jet Energy Corrections}

The jet clustering algorithm attempts to recover the four vector of the original parton. This is difficult because of two basic problems:

- Calorimeter response.

1. Nonlinearity in energy response.

2. Energy loss in un-instrumented detector area.

- Jet energy definition.

1. Underlying event energy inside the clustering cone.

2. Jet particles lost outside of the clustering cone.

It is thus desirable to correct the observed jet energy for effects due to the clustering algorithm and/or the detector response. The energy correction is obtained using CDF di-jet data and Monte Carlo simulation.[46]

The calorimeter jet energy response relative to the average central response is obtained by requiring $p_{T}$ balance in di-jet events. A scale factor is derived by comparing the $p_{T}$ of the central jet to the $p_{T}$ of the other jet, as a function of the $\eta$ of the other jet. The absolute central jet energy response is obtained using a Monte Carlo simulation. Gluon pairs are generated flat in $p_{T}$ and $\eta$. Event simulation, including fragmentation, underlying event insertion, and detector response is carried out as described in Section 5.3. The simulation is then tuned to reproduce CDF di-jet data. The jets are reconstructed using the standard 
algorithm. The simulated jet cluster is matched to the original gluon, defined as one within a radius of 0.6 in $\eta \phi$. The calorimeter response, as a function of $p_{T}$, is the ratio of the sum $p_{T}$ of particles inside the jet cone to the jet cluster $p_{T}$.

The energy from the underlying event is measured using CDF di-jet data, as the energy inside a central jet cone $90^{\circ}$ away from the leading jet's axis in $\phi$. But since the absolute energy response is determined with a Monte Carlo that includes underlying event, the underlying event energy must be corrected for energy response before subtracting it from the response-corrected jet energy. This correction is obtained, as in the jet energy response determination, by comparing the sum $p_{T}$ of calorimeter towers in a jet cone $90^{\circ}$ away from the leading jet in $\phi$ to the sum $p_{T}$ of the generated particles in that same cone. Because of the ambiguity in distinguishing underlying event particles from fragmentation particles, the underlying event correction must be applied in conjunction with an out-of-cone correction. First, the difference between the sum $p_{T}$ of particles in the jet cone and the total $p_{T}$ of the matched parton is determined. Then, to account for underlying event over-subtraction, the sum $p_{T}$ of fragmentation particles in the $90^{\circ}$-away cone is added to this difference to result in a correction for jet particles lost outside of the clustering cone. In summary the observed jet energy is corrected as follows:

$$
\begin{aligned}
E_{T}(\text { corrected })= & E_{T}(\text { observed }) \times(\text { absolute central jet energy scale }) \\
& \times(\text { relative jet energy scale }) \\
& -(\text { corrected underlying event energy }) \\
& +(\text { jet particles out-of-cone lost })
\end{aligned}
$$

The corrected jet energy depends on a particular fragmentation model. For results presented here, comparisons are made directly with Monte Carlo predictions that include various detector effects, and jet energy correction is not necessary. We only use jet energy correction when studying the missing $E_{T}$ distributions. 


\subsection{Jet Selection Efficiency}

The efficiencies of our jet clustering algorithm and the selection cuts are not perfect. Fluctuations in the underlying event energy deposition could lead to fake clusters. On the other hand, jet clusters below the $E_{T}$ threshold are not counted. However, we compare data with QCD predictions after Monte Carlo simulation of experimental effects. These effects are accounted for in the predictions.

But the effect from "Texas Towers", energy clusters from slow neutrons, is not simulated. The "Texas Towers" are removed in a cleanup procedure.[20] Studies indicate that $>95 \%$ of the "Texas Towers" above $20 \mathrm{GeV}$ in energy and not overlapping with a jet are eliminated.[47] We scan our data sample and find no evidence of "Texas Towers" being identified as a jet. We cannot, however, exclude the "Texas Towers" inside a jet. This is treated as leading to an additional uncertainty in the jet energy scale, which directly affects jet counting since we make an $E_{T}$ threshold requirement. This contributes an uncertainty in the jet multiplicity measurement.

\subsection{Comparison of Data with QCD Expectations}

We now compare the distributions observed in the data with theoretical expectations. The QCD prediction is given by the Papageno Monte Carlo calculation. We use QFL to simulate the calorimeter response to jets, and a simple model to simulate the $Z$ selection process. The theoretical cross sections, obtained from Papageno plus event simulation, is multiplied by the integrated luminosity and the $Z \rightarrow e e(\mu \mu)$ branching fraction, to give the expected number of events. Experimental effects such as acceptance and detector response are simulated in the Monte Carlo calculation, and no correction to the data is necessary for the comparisons. The $Z+1,2$-jet events are generated with the following parton level cuts.

- The parton transverse momentum: $p_{T}>5 \mathrm{GeV} / c$.

- The parton pseudo-rapidity: $|\eta|<3.5$. 
- The separation between partons in $\eta \phi$ geometry: $\Delta R>0.7$.

The EHLQ [48] set 1 parametrizations of parton distributions are used.

Events are generated with one (two) parton(s) in the final state for the $Z+1$-jet $(Z+2$ jet) mode. Ideally, one would use the $n$-jet matrix elements, $n=0,1,2, \ldots$, calculated to all orders in $\alpha_{s}$ to generate events with a mixture of jet multiplicities, and predict the $Z+1$-jet and $Z+2$-jet rates after event simulation. However, only the leading order matrix elements are available in Papageno and combining cross sections at different orders of $\alpha_{n}$ is inconsistent with perturbative calculations. Thus, the $Z+1$-jet rate is predicted using only the 1-jet matrix elements. That is, it does not include contributions from events with two (or more) partons in the final state where only one parton is detected as a jet. Similarly, the $Z+2$-jet rate is predicted using only the 2 -jet matrix elements. This is a fundamental limitation on the predicative power of leading order QCD calculations such as those used in the Papageno Monte Carlo.

\subsubsection{Jet Production}

The distributions of the $Z p_{T}$, the jet $E_{T}, \eta$, and EM energy fraction are compared with QCD predictions in Figure 5.5 for $Z+1$-jet events. The same distributions are shown in Figure 5.6 for $Z+2$-jet events, where the jet variables are for the second most energetic jet. We combine the $Z \rightarrow e e$ and $Z \rightarrow \mu \mu$ data samples for better statistics. There are good agreements in both the normalization and the shape of these distributions between data and theory.

The invariant mass of the two jets could be used to search for signals of resonance decaying into two jets. The comparison of the jet-jet invariant mass distribution between data and theory is shown in Figure 5.7. We are limited by the small number of 2 -jet events in our sample. But the QCD expectation adequately describes the data.

It is also interesting to measure the jet multiplicity in these $Z$ events. There are three events with more than two jets in the event. Their event properties are summarized in Table 5.1. The results of 0 to 4 -jet production rates are summarized in Table 5.2 and shown 


$$
\mathrm{Z}+1-\mathrm{Jet}
$$
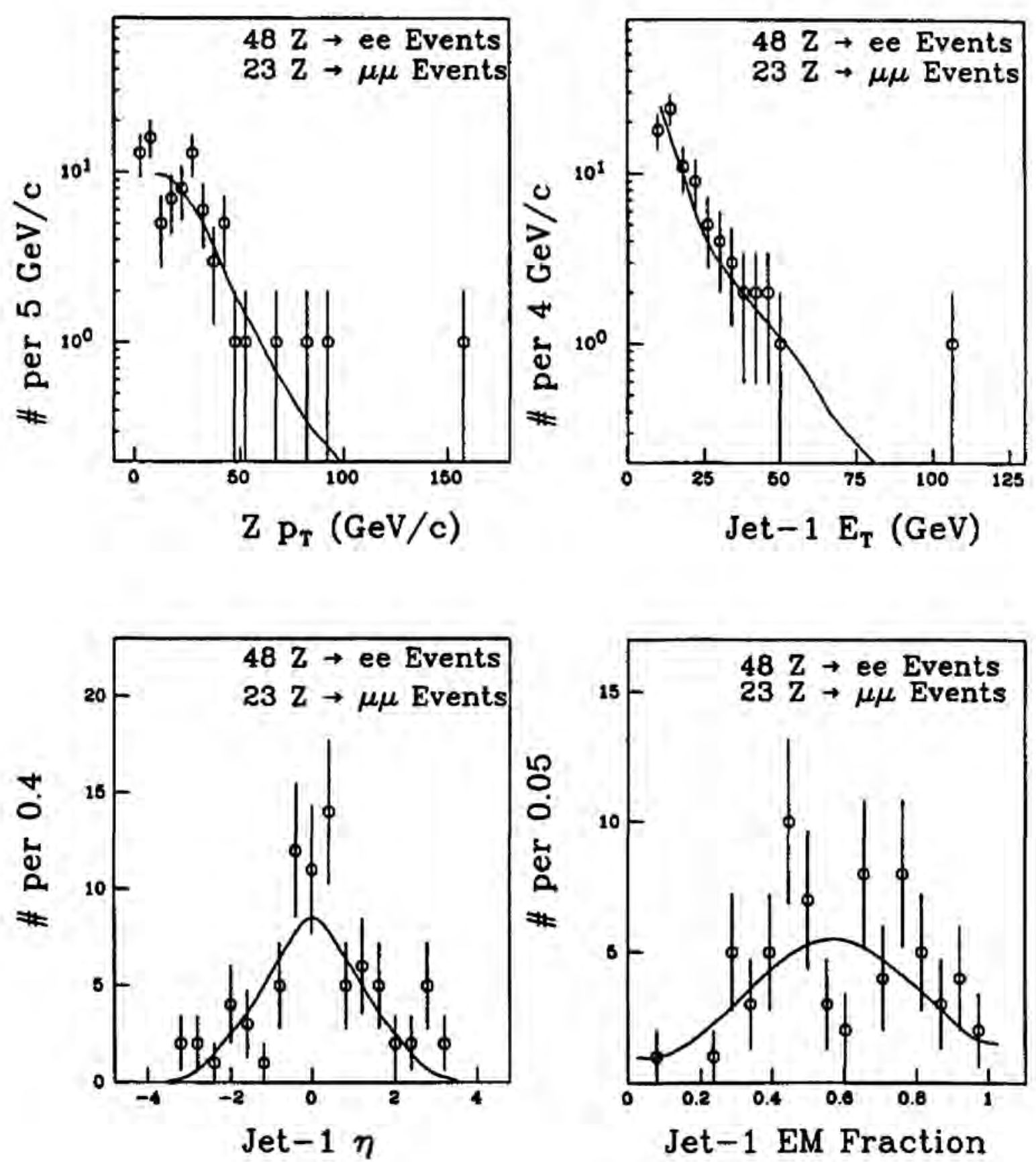

Figure 5.5: The $Z+1$-jet event properties compared with QCD predictions. The curves are Papageno plus QFL simulation for jet selection and a simple model for lepton selection. 


\section{$Z+2$-Jet}
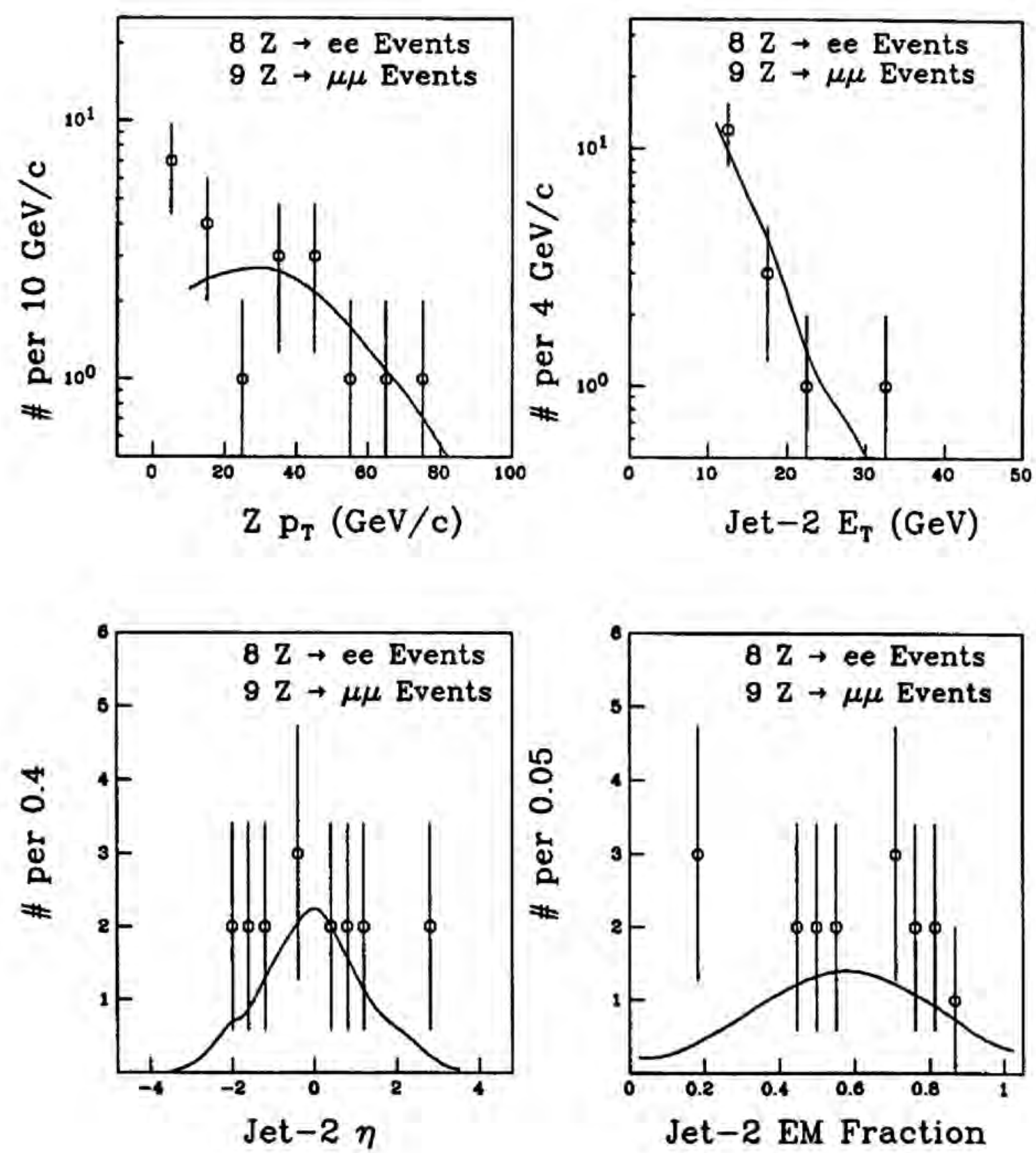

Figure 5.6: The $Z+2$-jet event properties compared with QCD predictions. The curves are Papageno plus QFL simulation for jet selection and a simple model for lepton selection. 
Table 5.1: The $Z$ events with high jet multiplicity

\begin{tabular}{l|ccc}
\hline Event & Jet $E_{T}(\mathrm{GeV})$ & Jet $\eta, \phi$ & Jet EM Fraction \\
\hline Run:16036, Event:164 & 33.5 & $0.2,278^{\circ}$ & 0.46 \\
$Z \rightarrow e e, 4$-jet, $p_{T}=9 \mathrm{GeV} / c$ & 33.3 & $-0.5,108^{\circ}$ & 0.18 \\
$\boldsymbol{\psi}_{T}=6 \mathrm{GeV}, \phi\left(\boldsymbol{\phi}_{T}\right)=74^{\circ}$ & 19.4 & $-1.9,338^{\circ}$ & 0.87 \\
& 12.7 & $-2.0,270^{\circ}$ & 0.38 \\
Run:17160, Event:8756 & & & \\
$Z \rightarrow e e, 3$-jet, $p_{T}=54 \mathrm{GeV} / c$ & 28.5 & $1.1,151^{\circ}$ & 0.25 \\
$\boldsymbol{\phi}_{T}=21 \mathrm{GeV}, \phi\left(\boldsymbol{E}_{T}\right)=168^{\circ}$ & 13.2 & $1.4,212^{\circ}$ & 0.79 \\
& 12.6 & $0.4,130^{\circ}$ & 0.39 \\
Run:19944, Event:84716 & & & \\
$Z \rightarrow \mu \mu, 3$-jet, $\boldsymbol{p}_{T}=66 \mathrm{GeV} / c$ & 15.6 & $-1.6,258^{\circ}$ & 0.51 \\
$\boldsymbol{\phi}_{T}=39 \mathrm{GeV}, \phi\left(\boldsymbol{Z}_{T}\right)=61^{\circ}$ & 10.1 & $-1.7,180^{\circ}$ & 0.19 \\
\hline
\end{tabular}

in Figure 5.8, along with various QCD predictions. Note that $n$-jet here means there are $n$ and only $n$ jets in the event. (That is, the 2 -jet events do not include 3 -jet events, etc.) The first uncertainty in the observed multiplicity is statistical. The second is due to the systematic uncertainty in the jet energy scale. This is the dominant systematic uncertainty since we make an $E_{T}$ cut in jet selection, and the jet $E_{T}$ distribution falls off rapidly. It is derived by varying the jet selection $E_{T}$ threshold of $10 \mathrm{GeV}$ by $\pm 15 \%$. That is, the upper uncertainty is obtained by setting the jet selection $E_{T}$ threshold at $11.5 \mathrm{GeV}$, the lower at 8.5 GeV. This is a conservative estimate, to account for uncertainty in the jet energy scale from calorimeter response and possible "Texas Tower" overlap with the jet.

The jet production rates are compared directly with Papageno plus event simulation in Table 5.2. This Monte Carlo calculation of the cross section is multiplied by $\mathcal{L}=4.05 \mathrm{pb}^{-1}$ and $B=0.033$ to yield the predicted rates.

We also make comparisons with predictions from parton level QCD calculations. The calculation of Brandt, et. al.,[49] uses the available complete $\mathcal{O}\left(\alpha_{n}^{2}\right)$ matrix calculation described in Section 1.3, that gives the sum of 1 -jet and 2-jet production. The 0 -jet cross section is given by the lowest order diagrams. A finite perturbative 2-jet cross section, normalized to the 0 -jet cross section, is calculated using the tree level diagrams at $\mathcal{O}\left(\alpha_{s}^{2}\right)$, along 


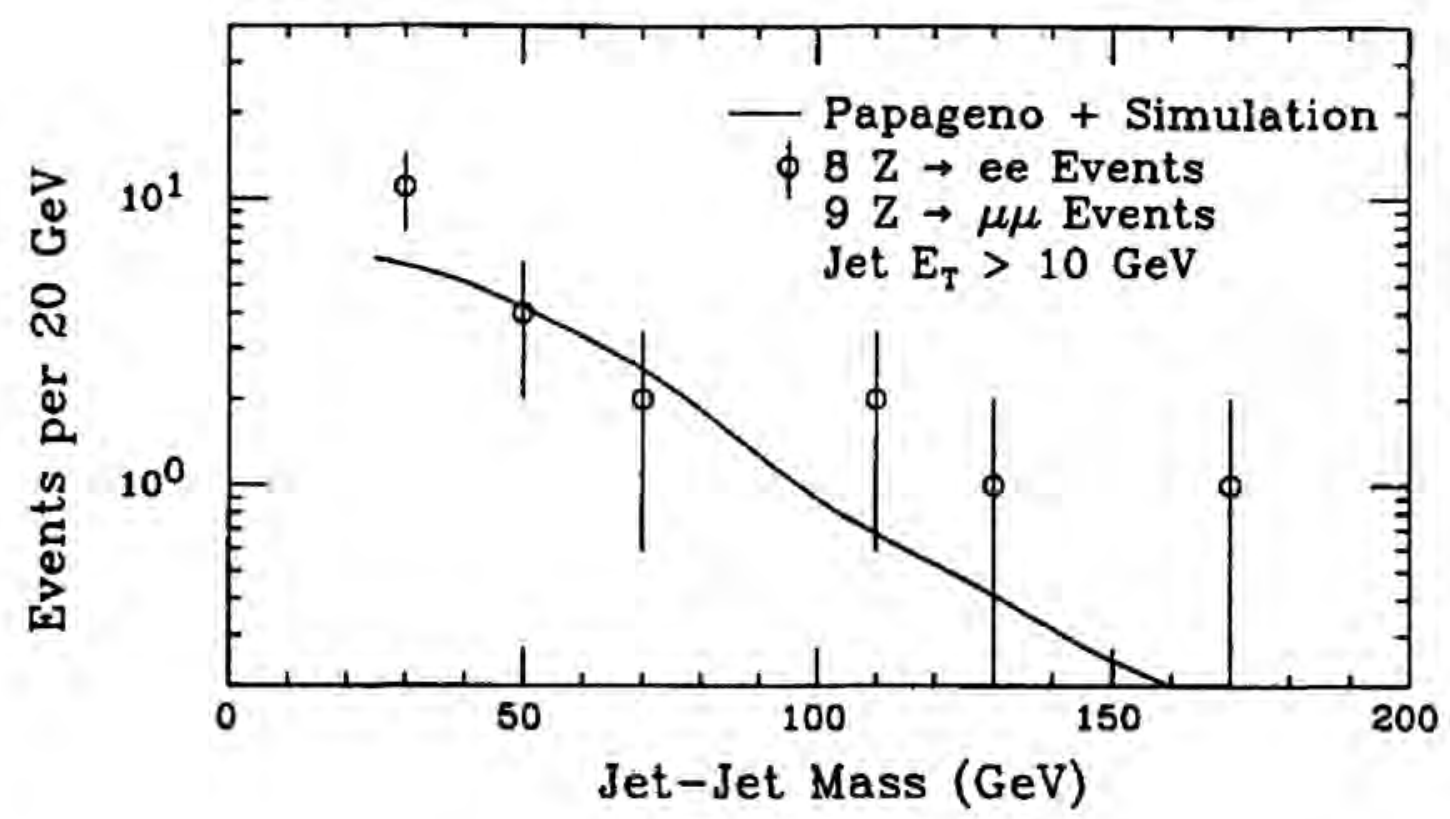

Figure 5. $\bar{i}$ : The jet-jet invariant mass distribution for $Z+2$-jet events. The curve is Papageno plus QFL simulation for jet selection and a simple model for lepton selection.

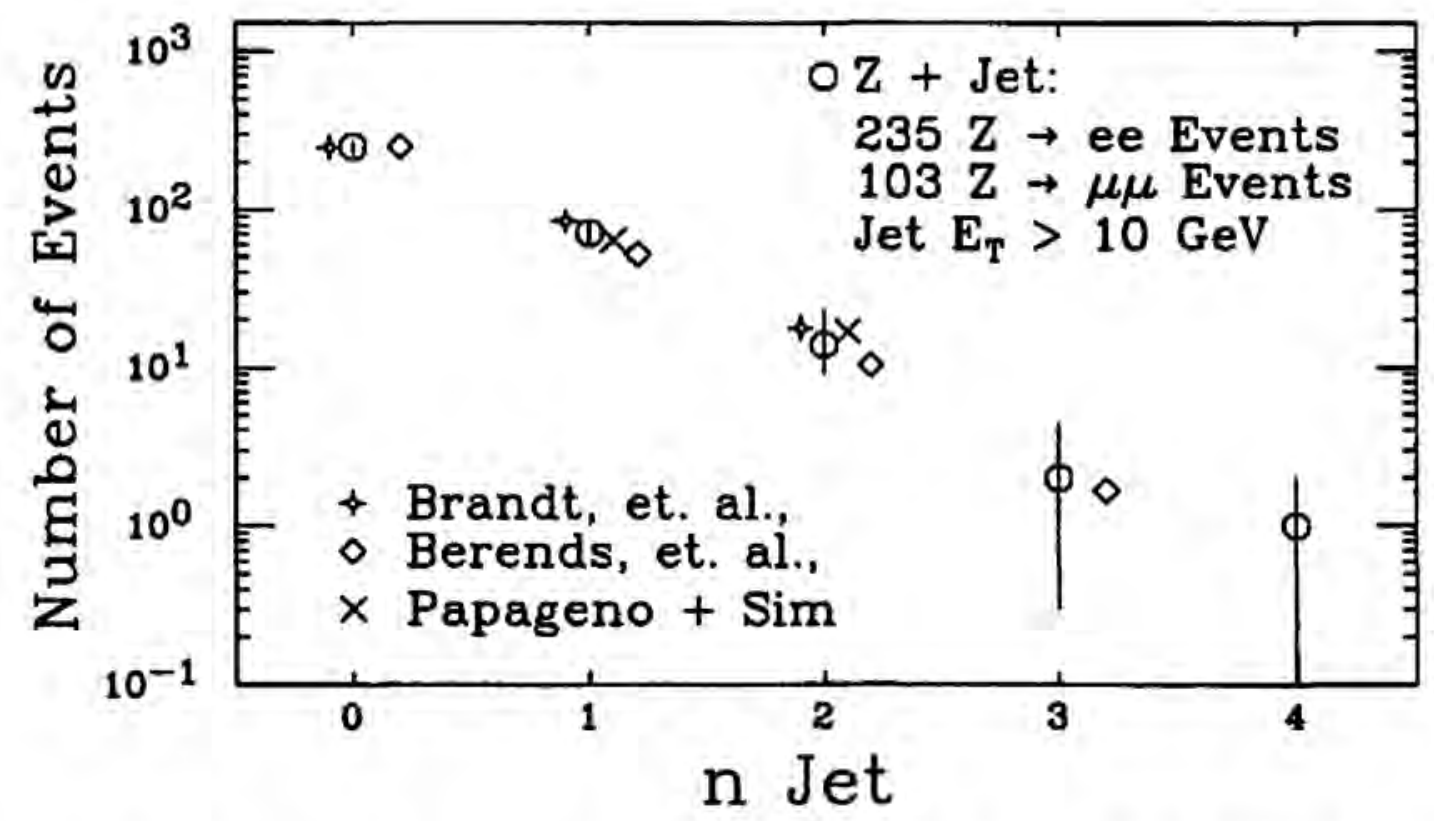

Figure 5.8: The Jet multiplicity in $Z$ events. The 0 -jet rate from Berends, et. al. is normalized to data. 
Table 5.2: The Jet multiplicity in $Z$ +jet events. The first uncertainty is statistical. The second is systematic, due to $\pm 15 \%$ uncertainty in the jet energy scale (see text). The 0 -jet rate from Berends, et. al. is normalized to data.

\begin{tabular}{lcccc}
\hline & Data & Papageno + Simulation & Brandt, et. al. & Berends, et. al. \\
\hline 0-jet & $250 \pm 16_{-20}^{+14}$ & - & 248 & 250 \\
1 -jet & $71 \pm 8_{+11}^{-8}$ & 64 & 84 & 52 \\
2-jet & $14 \pm 4_{+8}^{-4}$ & 17 & 18 & 10 \\
3-jet & $2 \pm 1_{+2}^{-1}$ & - & - & 1.7 \\
4-jet & $1 \pm 1_{0}^{0}$ & - & - & - \\
\hline
\end{tabular}

with parton level cuts to avoid singularities. Then, by subtracting the 2-jet cross section from the inclusive 1-jet plus 2-jet cross section, the $\mathcal{O}\left(\alpha_{s}^{2}\right)$ correction to 1-jet production is obtained. To compare these rates with data, an additional correction of $40 \%$ for the $Z$ selection acceptance, obtained from the Monte Carlo simulation, is applied.

A similar parton level calculation also exists from Berends, et. al..[50] The matrix elements for vector boson plus parton production are used, along with parton level cuts, to predict the jet multiplicity in $Z$ events. Their parton and lepton acceptance cuts are different from the ones we made in our data selection. The predicted 0 -jet rate is normalized to data, and only the relative jet production rates are compared.

These comparisons with parton level calculations are not precise, since the calculations do not take into account the effects of fragmentation or detector responses. They nevertheless provide a check of Papageno plus event simulation calculations. In any case, all QCD predictions agree with the data. 


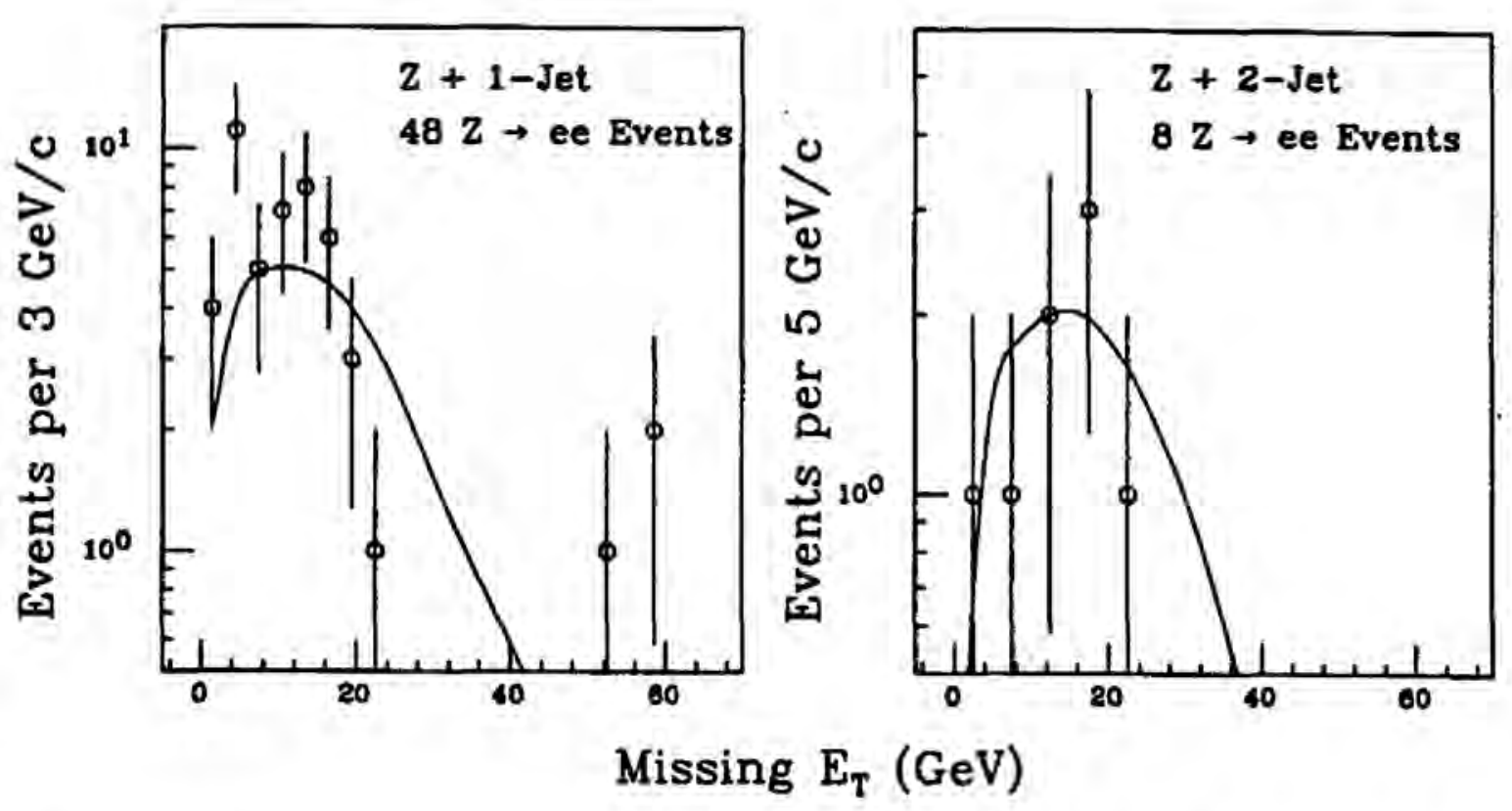

Figure 5.9: The uncorrected Missing $E_{T}$ in $Z$ events compared with QCD expectation. The curves are Papageno plus QFL simulation for jet selection and a simple model for lepton selection.

\subsubsection{The Missing $E_{T}$}

The observed (uncorrected) $F_{T}$ distributions in $Z \rightarrow e e$ events are compared with QCD expectation in Figure $5.9 .^{2}$ The observed $\not_{T}$ in $Z+1,2$-jet events is well described by Papageno plus event simulation, which predicts a tail in the distribution, most likely due to calorimeter energy measurement error.

In fact, a closer look at the three 1 -jet events with large $F_{T}$ reveals that the azimuthal direction of the $\mathbb{F}_{T}$ for each event is very close to that of a jet. A measurement error in the jet energy would cause an apparent large $\boldsymbol{Z}_{T}$. Jet energy correction is discussed in Section 5.4. We correct the observed missing $E_{T}$ for jet energy mis-measurement as follows:

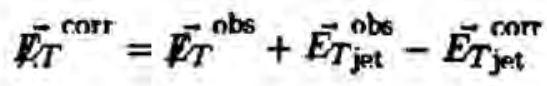

In Figure 5.10, we show the $p_{T}$ distributions before and after jet energy corrections. The

\footnotetext{
${ }^{2}$ For technical reasons, the $Z-\mu \mu$ events have not been simulated with QFL. As discussed in Section 5.2, the $Z_{T}$ in $Z-\mu \mu$ events behaves slightly differently from that in $Z-e \epsilon$ events.
} 


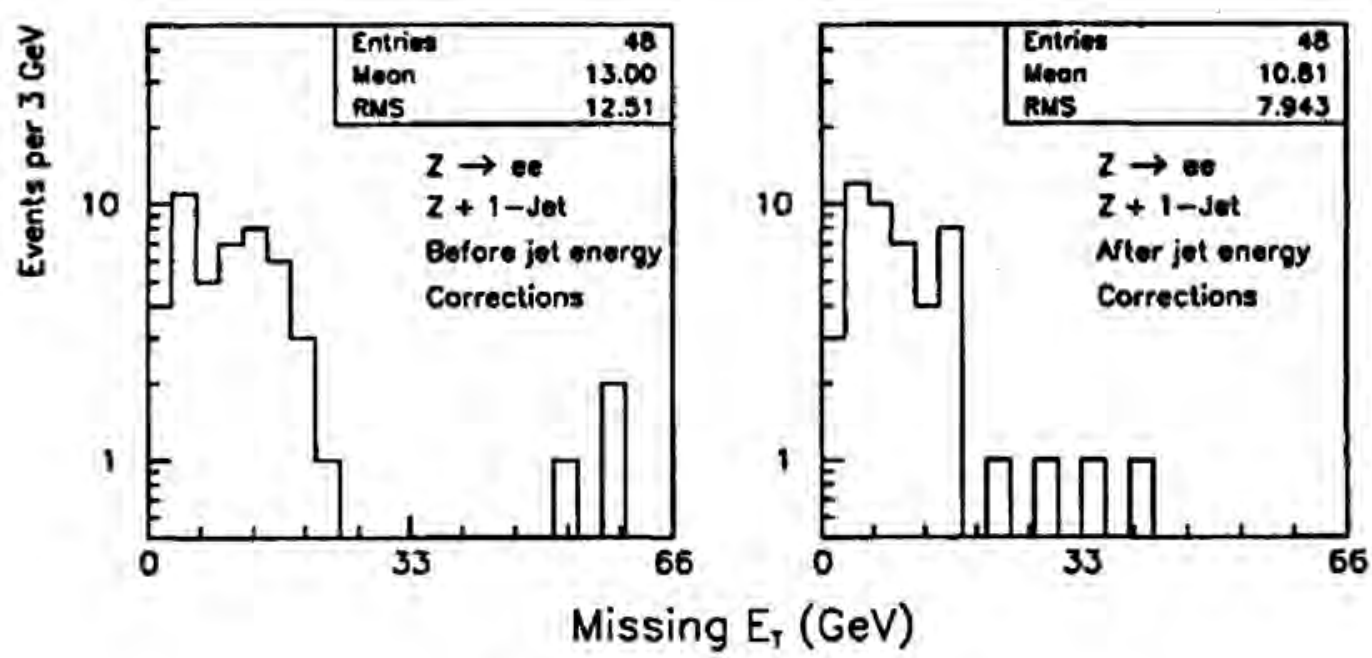

Figure 5.10: The $\not \not_{T}$ distribution in $Z \rightarrow e e$ events before and after jet energy correction.

large $D_{T}$ in those three events is shifted toward the lower end, as expected.

Another quantity of interest, and related to $\not_{T}$, is the $\vec{E}_{T}$ recoiling against the $Z \overrightarrow{p_{T}}$ defined as:

$$
\vec{E}_{T}^{\text {reroil }}=\sum_{i} E_{T}^{i} \cdot \hat{n}_{i}-\vec{E}_{T}^{\text {cal }} \text { (leptons). }
$$

That is, the vector sum of calorimeter energy, excluding the energy deposition from the $Z$ decay leptons. This is an alternative measure of the $Z p_{T}$. We show $E_{T}^{\text {recnil }}$ vs. $p_{T}$ in Figure 5.11. The data points are clustered around the diagonal dashed line, indicating a direct correlation between the magnitudes of these two quantities. Also shown is the same scatter plot with $E_{T}^{\text {rernil }}$ corrected for jet energy measurement as in Equation 5.4. Events with large missing $E_{T}$ appear as points far above the diagonal. 

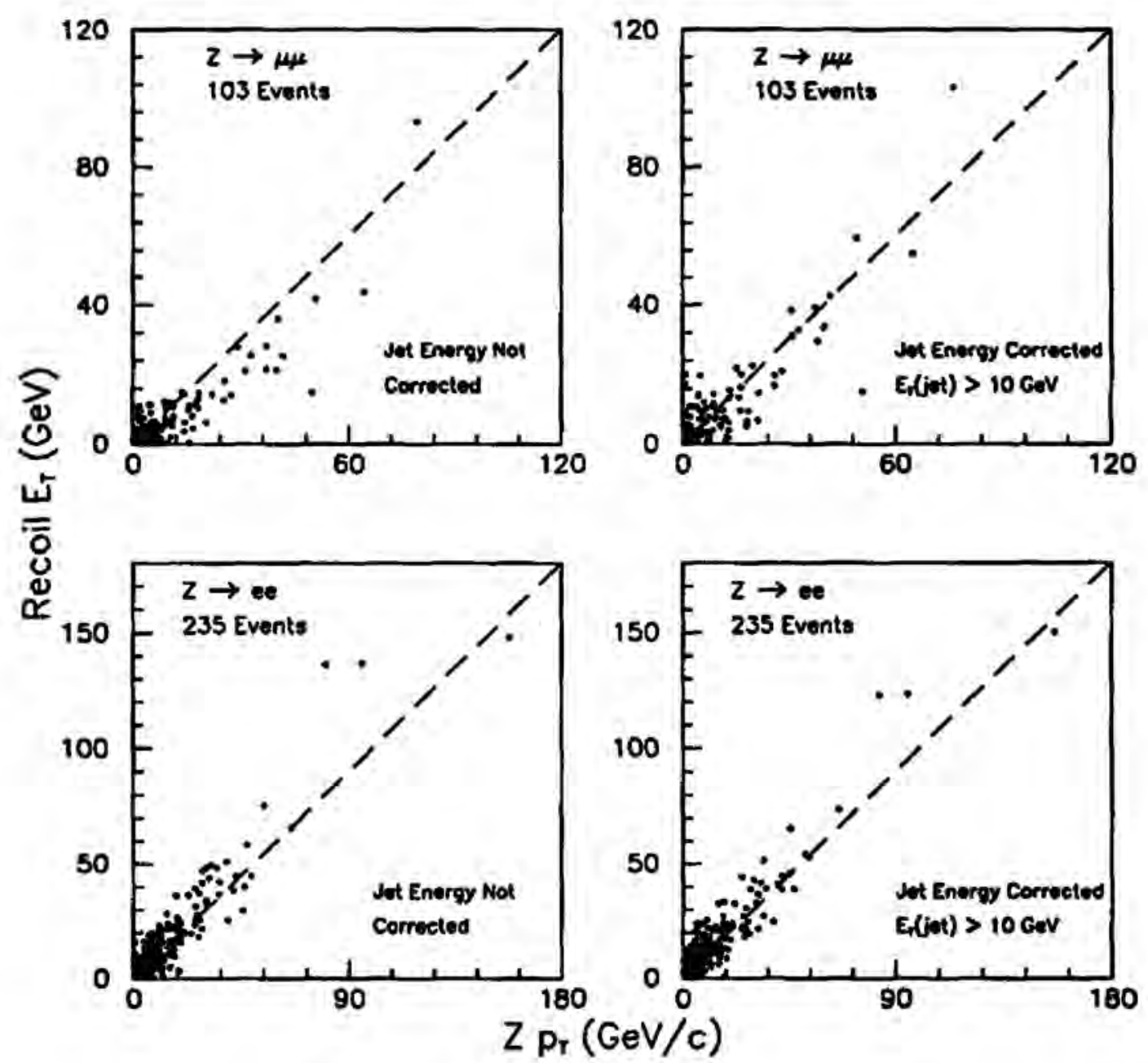

Figure 5.11: The $E_{T}$ recoiling against the $Z$ vs. the $Z p_{T}$. The $45^{\circ}$ dashed line is to guide the eye. 


\section{Chapter 6}

\section{Measurement of the $Z d \sigma / d p_{T}$}

Having examined the various aspects of our $Z$ event sample and concluded that they agree with our expectations, we now proceed to measure the inclusive $Z$ production differential cross section as a function of $p_{T}$. The measurement is made using information from the leptons. The transverse momentum of the $Z$ is the vector sum of the ee or $\mu \mu$ transverse momenta. We account for the inefficiency in the event selection procedure - the fact that the number of events observed does not correspond to the true number of events produced. We alsa correct for the $p_{T}$ dependent detector acceptance. Detector resolution also introduces

distortion in the observed $Z p_{T}$ spectrum. In this Chapter, we discuss the corrections for acceptance and resolution smearing in the $d \sigma / d p_{T}$ measurement.

\subsection{The Physics and Detector Models}

To understand the bias introduced in our kinematic and fiducial cuts, and the effect of resolution smearing, we need to model the detector as well as the physical process that we are studying. This is accomplished using a Monte Carlo simulation.

\subsubsection{The ISAJET Monte Carlo Event Generator}

$p \bar{p} \rightarrow Z \rightarrow e e(\mu \mu)$ events are generated using the ISAJET [41] Monte Carlo. We use several available structure function parametrizations, MRS [51] sets E and B and DFLM [52] sets 1,2 , and 3 in the event generation. In ISAJET, event generation starts with the hard scattering cross-section. Annihilation and Compton scattering diagrams and the full matrix 
elements for $Z$ decays are included. ISAJET has the feature that the $p_{T}$ spectrum agrees approximately with data. This is accomplished by choosing the cutoff used to remove the $1 / p_{T}^{2}$ singularity to reproduce approximately the $p_{T}$ spectrum calculated by resummation see Section 1.3. (But as discussed in Section 6.1.3, further adjustment to the Monte Carlo $p_{T}$ spectrum is needed to fit the data.) ISAJET also simulates other effects such as final state radiation and parton fragmentation. For the purpose of obtaining corrections for acceptance and resolution smearing, we only need the four-vectors of the Monte Carlo $Z$ decay leptons.

\subsubsection{The Simple Detector Simulation}

The $Z$ lepton detection and selection processes are simulated with a simple detector model. The electron energy resolution is easily modeled, as electromagnetic shower processes are well behaved, compared to the hadronic shower processes dominating jet energy resolution. The muon momentum resolution is given by the tracking resolution. The lepton identification efficiencies, as discussed in Section 4.1.3 and Section 4.2.3, are measured from the data using $Z$ decay leptons. The simple detector model is based on a set of input parameters. We vary the values of the model parameters within their uncertainties to obtain the uncertainty in the final $d \sigma / d p_{T}$ measurement due to uncertainty in the detector model. (See Section 7.2.) The model has the following features:

1. The event $z$-vertex distribution. It is modeled by by a Gaussian with $\sigma_{z}=30 \mathrm{~cm}$.

2. The electron fiducial region. The electron impact position at the calorimeter is determined by extrapolating its momentum vector to the detector.

3. The muon fiducial region. The muon impact position is determined by extrapolating the muon momentum vector to the detector taking into account the magnetic field.

4. The energy and momentum scales. The model assumes the energy and momentum scales are correctly set. (See Section 3.2.1 for discussion of CTC momentum scale, 
and Section 3.2.2 for discussion of electron energy correction.) The model uses the following scale uncertainties:

$$
\begin{array}{ll}
\text { CTC: } & \pm 0.1 \% \\
\text { CEM: } & \pm 0.4 \% \\
\text { PEM: } & \pm 5 \% \\
\text { FEM: } & \pm 5 \%
\end{array}
$$

\begin{tabular}{|c|c|c|c|c|c|}
\hline & $\left.\sigma_{E} / E\right)^{2}$ & & $\left(0.135 / \sqrt{E}_{T}\right)^{2}$ & + & (U) \\
\hline & $\sigma_{E} / E$ & & $0.28 / \sqrt{E}$ & & .02 \\
\hline & $\sigma_{E} / E$ & & $0.28 / \sqrt{E}$ & & .02 \\
\hline
\end{tabular}

5. The electron energy resolution. It is modeled by smearing the electron energy by a Gaussian with $\sigma$ given by the nominal calorimeter energy resolution:

The model uses the following conservative estimates of the uncertainties on the resolution:

\begin{tabular}{lcc} 
& $\sqrt{E_{T}}$ or $\sqrt{E}$ Term & Constant Term \\
\hline CEM: & $\pm 10 \%$ & $\pm 25 \%$ \\
PEM: & $\pm 10 \%$ & $\pm 50 \%$ \\
FEM: & $\pm 10 \%$ & $\pm 50 \%$
\end{tabular}

6. The CTC track momentum resolution. For beam-constrained tracks, it is $\left|\delta\left(\frac{1}{p T}\right)\right|=$ 0.0011 , with $p_{T}$ in $\mathrm{GeV} / \mathrm{c}$, or, $\delta p_{T} / p_{T}=0.0011 p_{T}$. Momentum resolution is modeled by smearing $\frac{1}{p T}$ by a Gaussian with $\sigma$ given by $0.0011 p_{T}$. An uncertainty of $\pm 10 \%$ on the parameter 0.0011 is used.

7. The lepton quality selection. The model does not actually simulate the detector response to leptons. We simply decide whether a given lepton passes our selection cuts based on the efficiency for selecting the given type of lepton. This amounts to randomly rejecting a fraction $(1-\epsilon)$ of the leptons whose identification efficiency is $\epsilon$. The values and uncertainties of lepton selection efficiencies are tabulated in Table 4.6 and Table 4.8 .

8. The lepton trigger requirement. This is done in the exact same way as for the previous item, based on the trigger efficiency of the given type of lepton. The values and uncertainties of the lepton trigger efficiencies are presented in Section 4.4.1 and Section 4.4.2. 
Note that this model does not include any bremsstrahlung effects. The electron energy is measured by the calorimeter. The effect of hard photon radiation on the acceptance is found to be small from a study including radiative effects.[32] It could have affected the acceptance through the mass window cut, as the energy resolution would have been different.

\subsubsection{Monte Carlo Simulation vs. Raw Data}

Before proceeding further, we check that the $Z$ production and decay properties observed in the raw data are well reproduced by the Monte Carlo simulation. The comparison is shown in Figures 6.1-6.2. The shape of the $p_{T}$ spectrum agrees well over the full range of observed data. (See below for more discussion on this aspect of the simulation.) The $Z$ decay properties are well reproduced, as shown in the distributions of lepton transverse momentum and pseudo-rapidity The fraction of events with a decay lepton in a given detector region is also properly reproduced. The cracks between the two halves of the CEM at $90^{\circ}$, the CEM and PEM at $30^{\circ}$, and the PEM and FEM at $10^{\circ}$ polar angles are correctly modeled, as can be seen in the electron $\eta$ distribution. Agreement in the $Z$ rapidity distribution shows that the longitudinal production property is also well modeled. The agreement in the mass distribution, shown in Figure 6.5, shows that momentum resolution smearing is properly modeled.

A slight discrepancy in the $Z p_{T}$ spectrum at low $p_{T}$ can be seen in Figure 6.1. The shape of the Monte Carlo $p_{T}$ spectrum could affect our $d \sigma / d p_{T}$ measurement through the resolution smearing corrections. We adjust the Monte Carlo $p_{T}$ spectrum to fit the data by accepting the appropriate fraction of Monte Carlo events in a given $p_{T}$ bin. This procedure is referred to as "sculpting":

1. Fit the raw $Z p_{T}$ spectrum to a parametrization

$$
\frac{\mathrm{dN}}{d p_{T}}=\frac{2 p_{T}\left(p_{T}^{2}-D\right)^{C-1}}{B\left(\left(E p_{T}\right)^{F}+1\right)},
$$

which is based on the function used in ISAJET to generate the boson $p_{T}$ distributions.[53] 


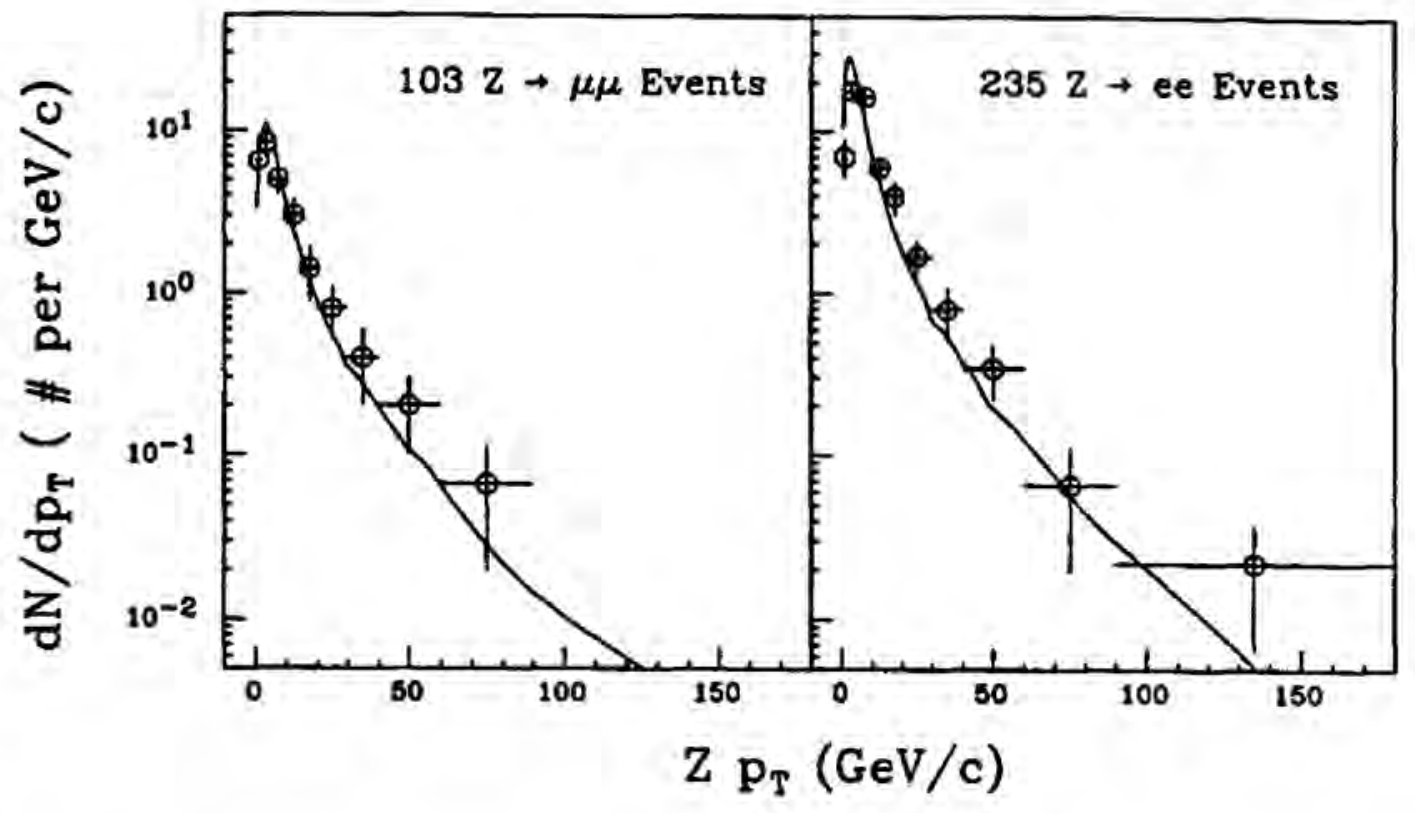

Figure 6.1: The observed (uncorrected) $Z p_{T}$ distributions. The curves are the defanlt Monte Carlo spectra normalized to data.

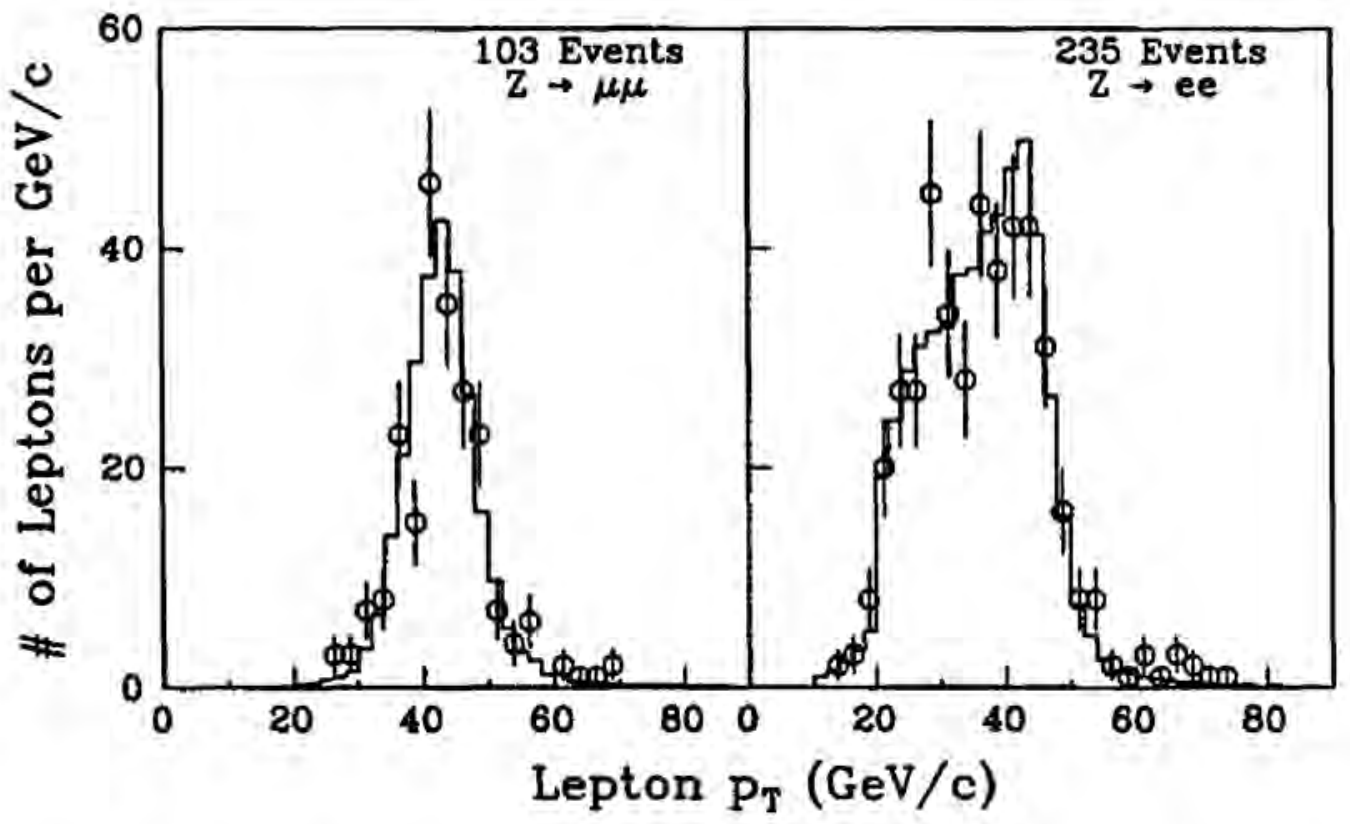

Figure 6.2: The lepton transverse momentum distributions. The histograms are the results of Monte Carlo simulations normalized to data. 


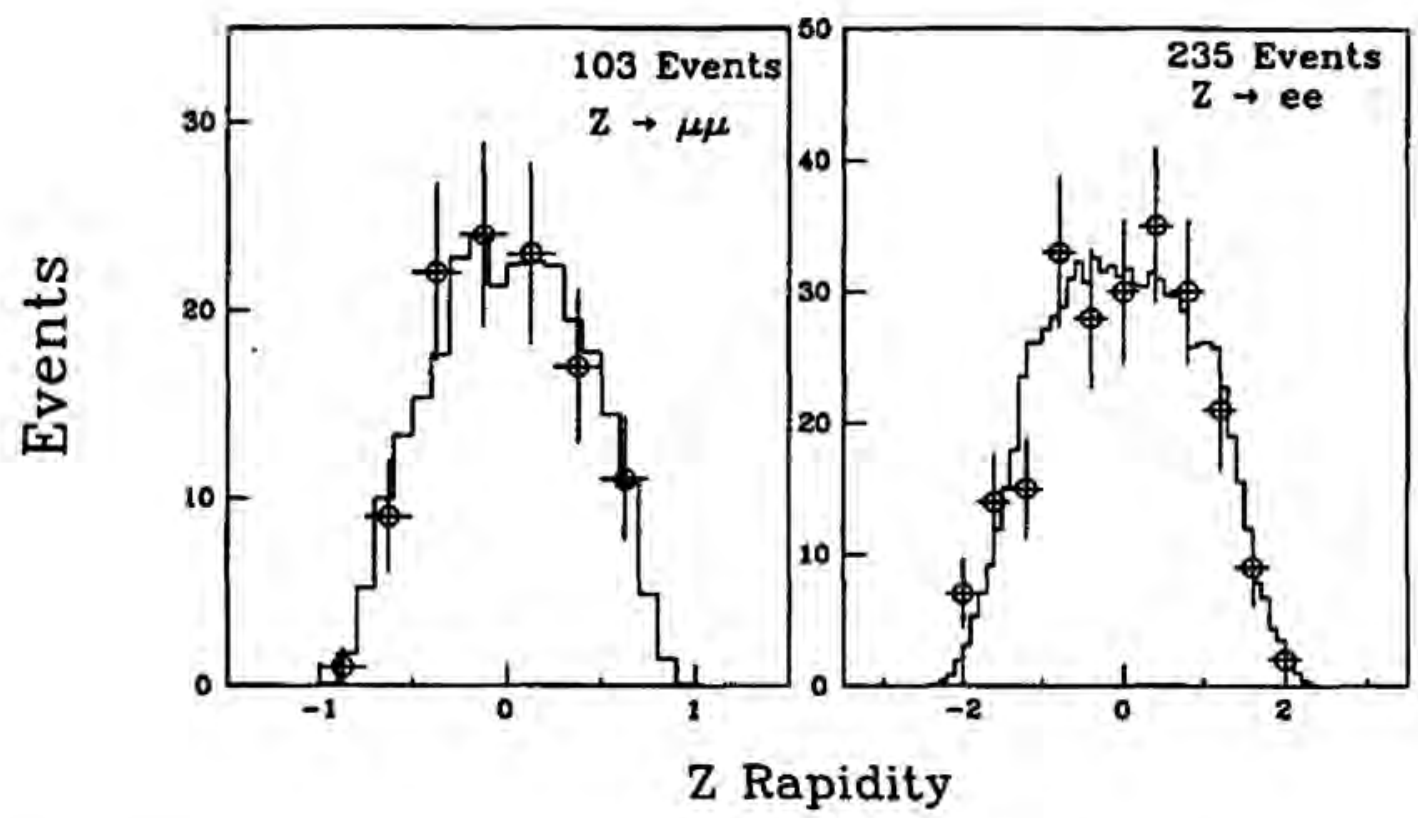

Figure 6.3: The $Z$ rapidity distributions. The histograms are the results of Monte Carlo simulations normalized to data.

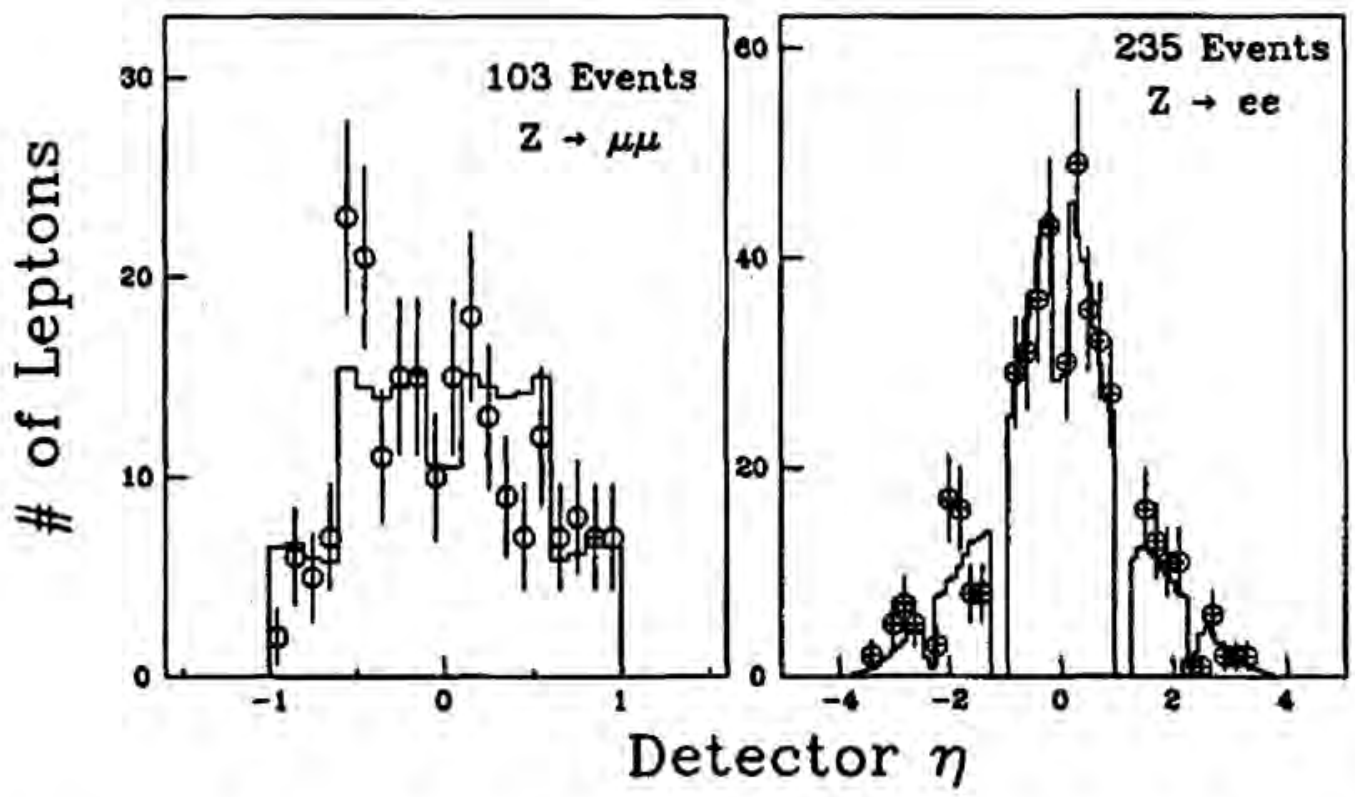

Figure 6.4: The lepton pseudo-rapidity distributions. The histograms are the results of Monte Carlo simulations normalized to data. 


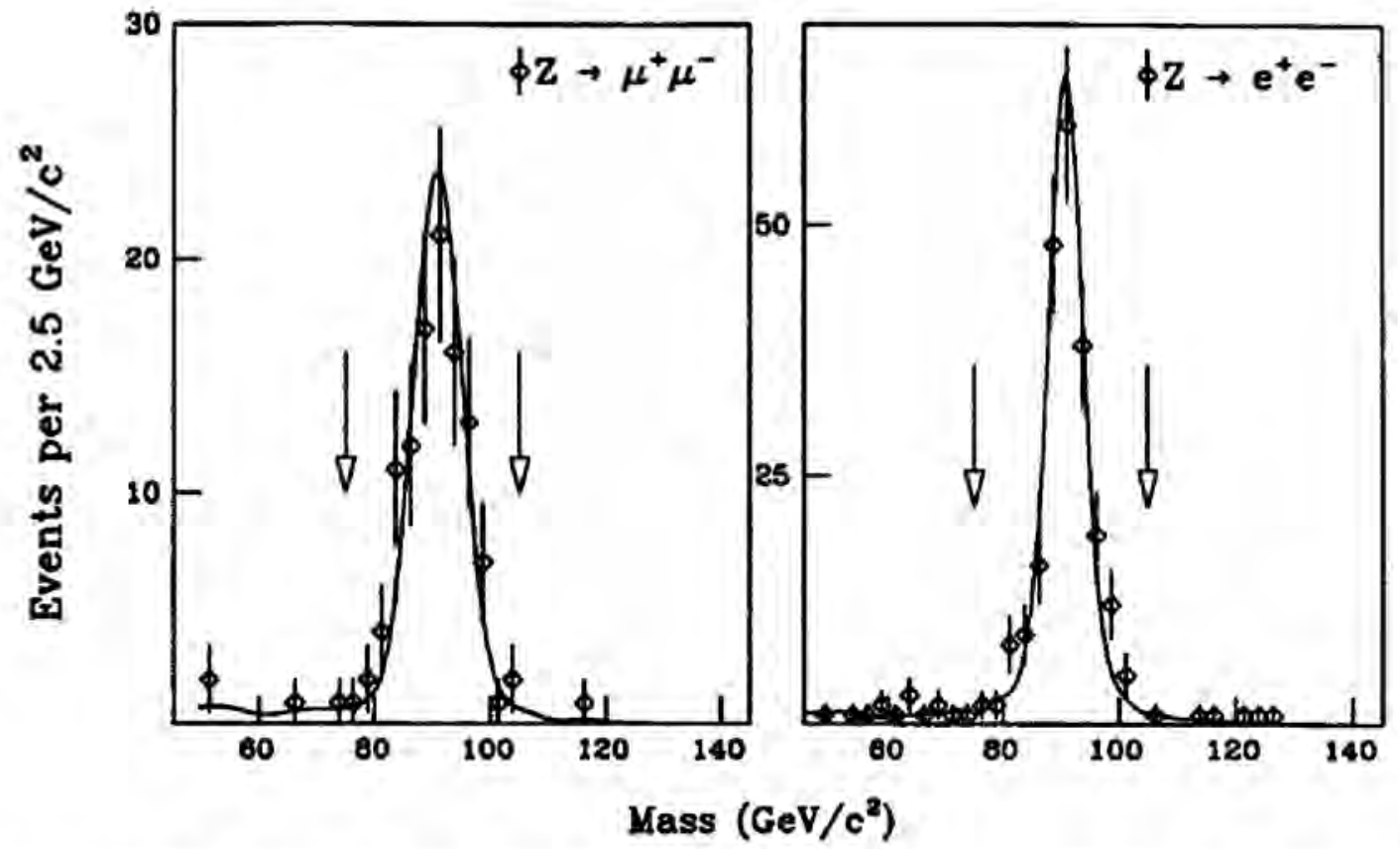

Figure 6.5: The invariant mass distributions of $\mu \mu$ and $e \epsilon$ pairs, jncluding low mass pairs. There are $103 Z \rightarrow \mu \mu, 235 Z \rightarrow e e$ candidate events with invariant mass between 75 and $105 \mathrm{GeV}$. The results of Monte Carlo simulations are shown as curves.

2. Fit the Monte Carlo $p_{T}$ spectrum, after detector simulation, to the same parametrization.

3. Obtain the function $S\left(p_{T}\right)$ as the ratio of the result of step 1 to the result of step 2 .

4. Then the factor

$$
\alpha\left(p_{T}\right)=\frac{S\left(p_{T}\right)}{\text { maximum of } S\left(p_{T}\right)}
$$

is the fraction of Monte Carlo events to be accepted at a given $p_{\boldsymbol{T}}$.

The results of this procedure are shown in Figure 6.6 for both the $Z \rightarrow e e$ and $Z \rightarrow \mu \mu$ data. The uncertainties in the fit parameters are used to estimate the systematic uncertainty in our measurement due to assumption in the input Monte Carlo $p_{T}$ spectrum. The Monte Carlo simulation is adequate and can be used to obtain acceptance and resolution corrections. We discuss the uncertainty due to assumptions in the Monte Carlo simulation in Section 7.2. 


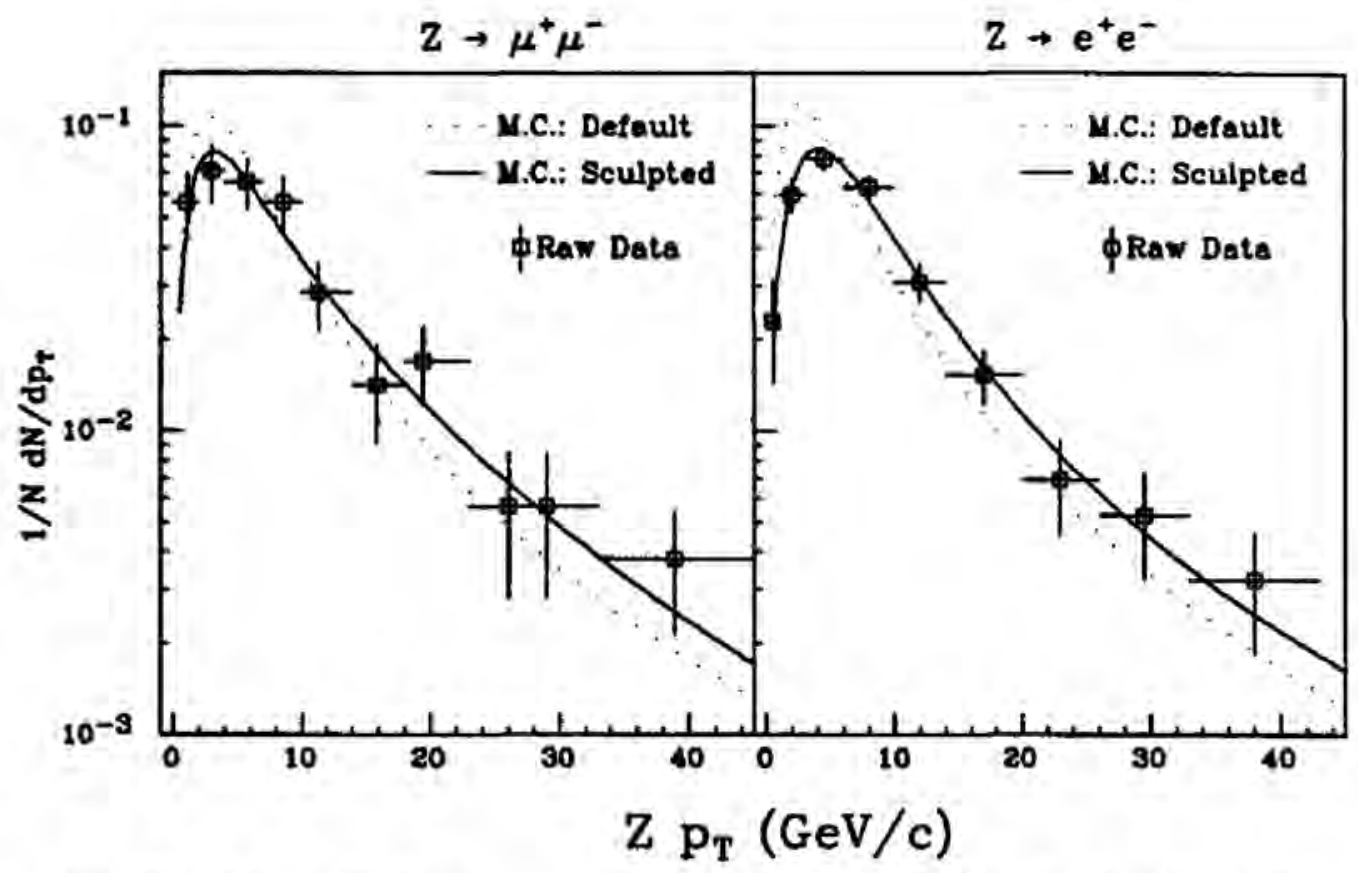

Figure 6.6: Two Monte Carlo $p_{T}$ spectra, one is the default, the other is adjusted to fit the raw data.

\subsection{The Acceptance Correction}

We measure the acceptance of the $Z$ selection procedure as a function of the $Z p_{T}$. For each Monte Carlo event, an event $z$-vertex is generated according to a Gaussian of width $\sigma=30 \mathrm{~cm}$. The efficiency of a cut at $2 \sigma$ is $\epsilon_{z v}=0.954$.

The fiducial cuts on Monte Carlo events are the same as those applied to data. For electrons, the cuts are:

1. $\mid z$-vertex $\mid<60 \mathrm{~cm}$,

2. at least one CEM electron, and

3. the electron momentum vector extrapolated to the calorimeter must be within fiducial regions defined in Table 4.1.

For muons, the cuts are:

1. $\mid z$-vertex $\mid<60 \mathrm{~cm}$, 
2. at least one muon with a drift chamber track (CMUO), and

3. the extrapolated muon momentum vector must be within the muon fiducial regions defined in Table 4.7.

The fiducial acceptance rises as a function of the $Z_{p_{T}}$, because the leptons from the decay of a high $p_{T} Z$ receive a large transverse boost toward the central region, and we always require a central lepton.

The selection cuts on Monte Carlo events passing the fiducial cuts reflect those applied to data:

1. The lepton trigger must be satisfied.

2. The lepton passing tight quality cuts must have at least $20 \mathrm{GeV}$ of transverse momentum. The lepton passing loose quality cuts must have at least $10 \mathrm{GeV}$ of transverse momentum.

3. The invariant mass of the two leptons must be between 75 and $105 \mathrm{GeV}$.

Note that this procedure accounts for the correlation between the $Z$ selection efficiency and the fiducial acceptance. The selection efficiency is found to be uniform as a function of the $Z p_{T}$. The acceptance, defined as the fraction of Monte Carlo events that passed all the above fiducial and selection cuts, is

$$
a\left(p_{T}\right)=\frac{\mathrm{N}(\text { pass fiducial and selection cuts })}{\mathrm{N}(\text { generated })} .
$$

The procedure described above can be used in separate measurements using the $Z \rightarrow e e$ or $Z \rightarrow \mu \mu$ samples. It can also be used in a combined measurement using both the $Z \rightarrow e e$ and $Z \rightarrow \mu \mu$ samples. We simply choose the $Z$ decay lepton type according to the branching fraction. We also account for the different effective luminosities for the two samples. The muon trigger was not working during the early parts of the run, and the data from those runs are not included. This leads to an effectively smaller $Z \rightarrow \mu \mu$ acceptance. Since in Equation 6.3 the denominator is the same for the two modes, the combined acceptance is 
simply the sum of the two. This is justified by a maximum likelihood argument given below. The $Z \rightarrow$ e. and $Z \rightarrow \mu \mu$ combined acceptance is shown in Figure 6.7 as a function of $p_{T}$.

\subsubsection{Combining Data Samples with Different Acceptances}

The $Z \rightarrow e e$ and $Z \rightarrow \mu \mu$ samples are combined in the final measurement for better statistical accuracy. We discuss how the acceptances for these two different samples are combined by considering the case where a cross section is measured by several experiments with different acceptances and statistics.

Let

- $\epsilon_{i}$ be the acceptance of the $i^{\text {th }}$ experiment,

- $\bar{n}_{i}=\epsilon_{i} \times \sigma$ be the average number of events this experiment expects to observe, where $\sigma$ is the true cross section this experiment attempts to measure,

then the probability to observed $n_{i}$ events is given by Poisson statistics:

$$
P\left(n_{i}, \bar{n}_{i}\right)=\frac{\bar{n}_{i}^{n_{i}} \times e^{-\bar{n}_{i}}}{n_{i} !}
$$

The joint probability for obtaining the results from all experiments is expressed in terms of the likelihood function:

$$
\mathcal{L}=\prod_{i} P\left(n_{i}, \bar{n}_{i}\right)
$$

The result of maximizing the log-likelihood,

$$
\begin{aligned}
0 & =\frac{\partial \ln \mathcal{L}}{\partial \sigma} \\
& =\frac{\partial}{\partial \sigma} \sum_{i} n_{i} \ln \left(\bar{n}_{i}\right)-\bar{n}_{i}-\ln n_{i} ! \\
& =\sum_{i} \frac{n_{i}}{\sigma}-\epsilon_{i}
\end{aligned}
$$

is

$$
\sigma=\frac{\sum_{i} n_{i}}{\sum_{i} \epsilon_{i}}
$$

That is, to combine the results of several experiments for a cross section measurement, the number of events observed should be added, and the acceptances are also added. This 


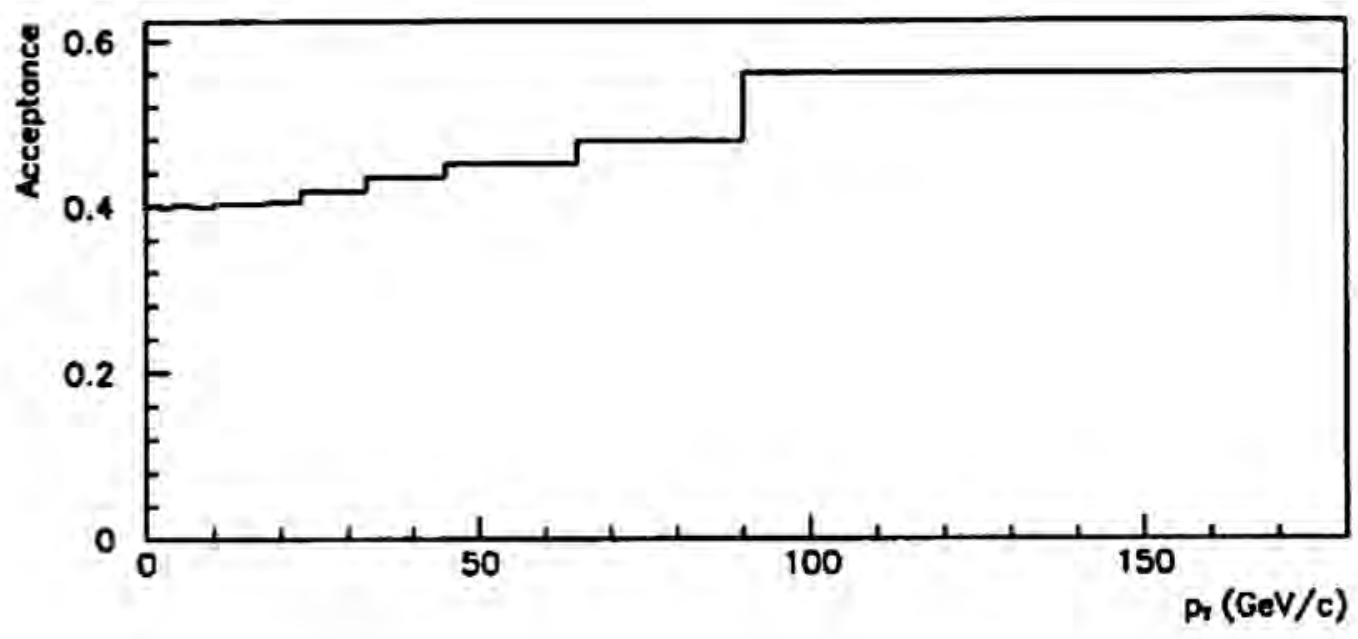

Figure 6.7: The combined $Z \rightarrow e e$ and $Z \rightarrow \mu \mu$ acceptance as a function of $p_{T}$.

justifies our Monte Carlo procedure described above for obtaining the acceptance for the $Z \rightarrow e e$ and $Z \rightarrow \mu \mu$ combined measurement.

\subsection{The Correction for Resolution Smearing}

Because of the intrinsic resolution in the lepton momentum measurement, the $Z p_{T}$ measured from the lepton momenta has an associated random error. This resolution smearing effect, together with a rapidly changing $p_{T}$ spectrum, leads to distortion in the observed spectrum. We correct for this smearing effect in the observed spectrum to obtain a $d \sigma / d p_{T}$ that can be directly compared to theoretical prediction.

The effect of resolution smearing can be expressed as the convolution of the detector resolution function and the true spectrum:

$$
f^{\prime}\left(p_{T}^{\prime}\right)=\int c\left(p_{T}^{\prime}, p_{T}\right) \cdot f\left(p_{T}\right) d p_{T}
$$

where

- $p_{x}$ is the true value of the transverse momentum, 
- $p_{x}^{\prime}$ is the observed value (with resolution smearing),

- $f\left(p_{T}\right)$ is the true spectrum $\left(d \sigma / d p_{T}\right)$,

- $c\left(p_{T}^{\prime}, p_{T}\right)$ is the detector resolution function, and

- $f^{\prime}\left(p_{T}^{\prime}\right)$ is the observed spectrum.

Note that the the resolution function $c\left(p_{T}^{\prime}, p_{T}\right)$ defined this way depends only on the lepton momentum resolution, that is, the electron energy resolution for $Z \rightarrow e e$, and the track momentum resolution for $Z \rightarrow \mu \mu p_{T}$ measurement.

In the case of limited statistics, as in this experiment, the observed spectrum is not a continuous distribution but a binned histogram. The analog of Equation 6.8 for the case of discrete $p_{T}$ values is:

$$
F_{i}\left(p_{T i}^{\prime}\right)=\sum_{j} C_{i j}\left(p_{T i}^{\prime}, p_{T j}\right) \cdot F_{j}\left(p_{T j}\right)
$$

where

- $F\left(p_{T i}\right)$ is the $i^{\text {th }}$ bin of the observed spectrum,

- $F_{j}\left(p_{T j}\right)$ is the $j^{t h}$ bin of the true spectrum, and

- $C_{i j}\left(p_{T i}^{\prime}, p_{T j}\right)$ is the resolution smearing matrix. The $i j^{\text {th }}$ element of this matrix is the probability that $p_{T_{j}}$ is measured as $p_{T ;}^{\prime}$ because of the detector resolution.

Then, if $C_{i j}$ is known, we can invert it, and use its inverse, $C_{i j}^{-1}\left(p_{T i}^{\prime}, p_{T j}\right)$, and $F_{i}^{\prime}$ to obtain $F_{j}$ :

$$
F_{j}=\sum_{i} C_{i j}^{-1} \cdot F_{i}^{\prime}
$$

Note, however, the $C_{i j}$ defined this way depends on the shape of the spectrum inside the bins. This is an artifact of finite bin widths, and is unavoidable for a measurement based on finite statistics.

From now on, we refer to Equation 6.9 as "smearing", $F$ ' the "smeared spectrum", Equation 6.10 as "unsmearing", and $F_{j}$ the "unsmeared spectrum". 
We obtain $C_{i j}$ using the Monte Carlo simulation described in Section 6.1. The electron energy resolution and the track momentum resolution are discussed in Section 6.1.2, and checked with data in Section 6.1.3. We derive the smearing matrix as follows:

1. Smear the lepton momentum with the resolution given in Section 6.1.2.

2. Find the relationship between $p_{T}$ and $p_{T}^{\prime}$ reconstructed from the Monte Carlo leptons. This is accomplished by accumulating a 2-dimensional histogram, with $p_{T}$ on one axis and $p_{T}^{\prime}$ on the other. Let $i$ label the rows for $p_{T}^{\prime}$, and $j$ label the columns for $p_{T}$. We call this 2-d histogram $A_{i j}$.

3. The smearing matrix is obtained by normalizing $A_{i j}$ :

$$
C_{i j}=\frac{A_{i j}}{\Sigma_{i} A_{i j}}
$$

Thus, for the $j^{\text {th }}$ column of $C_{i j}$, the $i^{\text {th }}$ element is the probability that $p_{T j}$ is measured as $p_{T i}^{\prime}$ because of detector resolution smearing.

The effective $p_{T}$ resolution, defined as the rms spread in the difference between $p_{T}$ and $p_{T}^{\prime}$, is shown as a function of $p_{T}$ in Figure 6.8. As expected, the resolution for the $Z \rightarrow e e$ is better than that for the $Z \rightarrow \mu \mu$ since the electron energy resolution is better than the CTC track momentum resolution. To show how big the smearing is, a few columns of $C_{i j}$ are shown in Figure 6.9. (The $p_{T}$ resolution is for the combined $Z \rightarrow e e$ and $Z \rightarrow \mu \mu$ measurement.) Figure $6.9 \mathrm{a}$ shows that for the $p_{T}$ bin 0 to $2 \mathrm{GeV}, \approx 50 \%$ of the time the measured $p_{T}^{\prime}$ is also in the range 0 to $2 \mathrm{GeV}$, and $\approx 35 \%$ of the time the measured $p_{T}^{\prime}$ is in the next bin, in the range 2 to $4 \mathrm{GeV}$.

We invert $C_{i j}$ to get $C_{i j}^{-1}$. A few rows of the $C_{i j}^{-1}$ are shown in Figure 6.10. The physical interpretation of each element of $C_{i j}^{-1}$ is less direct. But the histograms show how much "unsmearing" there should be for the content of each observed bin. For example, Figure $6.10 \mathrm{~b}$ shows that to obtain the "unsmeared" bin $\left(F_{2}\right)$, with $p_{T}$ between 2 and 4 $\mathrm{GeV}$, there are contributions from the original bin as well as the neighboring bins:

$$
F_{2}=3 \times F_{2}^{\prime}+(-2) \times F_{1}^{\prime}+(-0.1) \times F_{3}^{\prime}+\cdots
$$




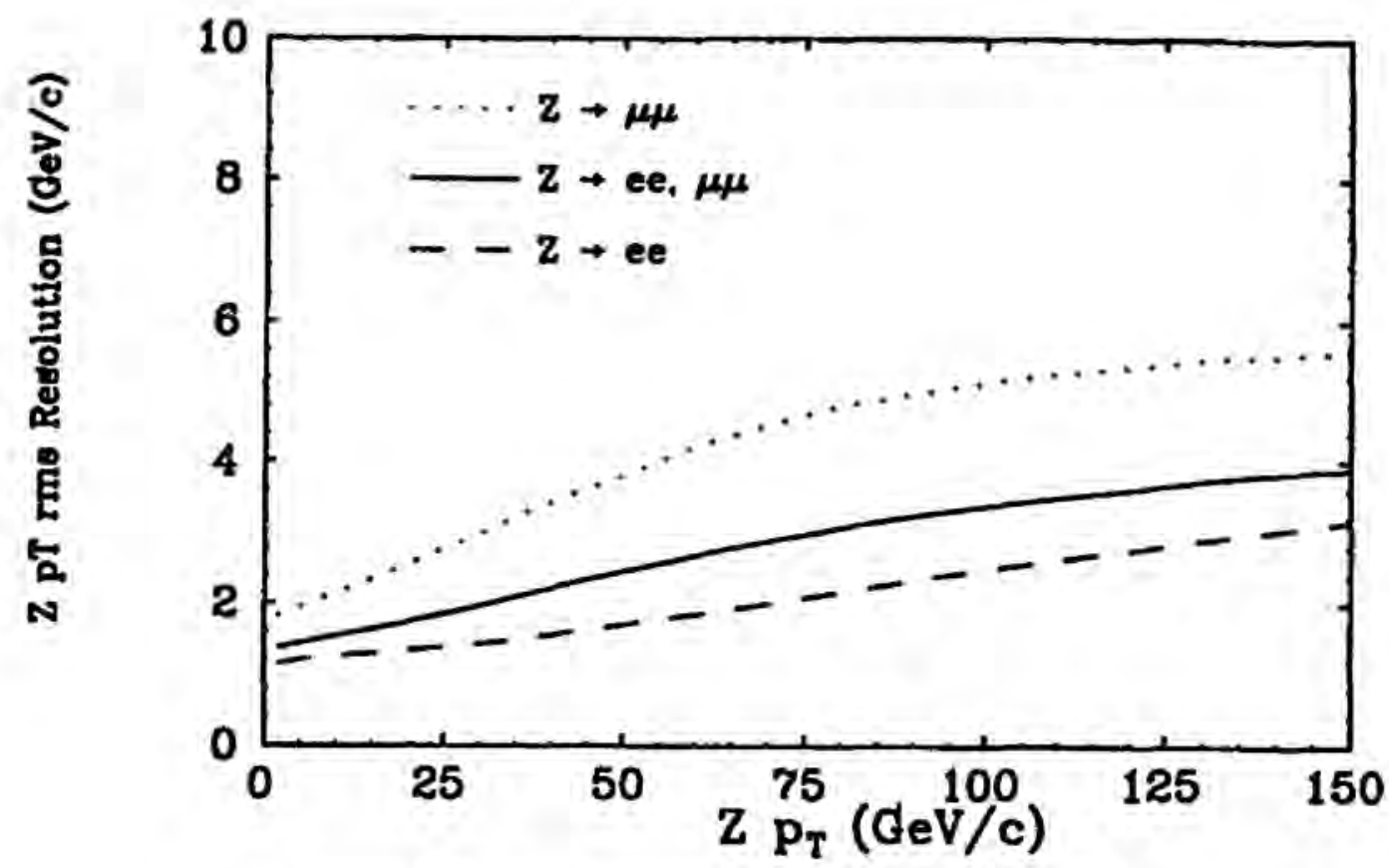

Figure 6.8: The $Z p_{T}$ resolution as a function of $p_{T}$.

The smearing matrix $C_{i j}$ is shown in Table 6.1.

To make sure that these matrices are properly constructed, we test them using the Monte Carlo simulation. We smear the input ISAJET $p_{T}$ spectrum in two distinct ways. First we smear it as we did in our usual procedure, described in Section 6.1.2, by the known lepton momentum resolution. This is the histogram shown in Figure 6.11a, and labeled as " $\sigma_{\mathrm{E}}$ smearing". We also smear it using the smearing matrix derived above. This is shown as the plotted circles in Figure 6.11a. We arrive at the same smeared spectrum with these two different smearing methods. Similarly, we test $C_{i j}^{-1}$ by comparing the never-smeared spectrum with the spectrum "unsmeared" using $C_{i j}^{-1}$ in Figure $6.11 \mathrm{~b}$. We see that our "unsmearing" procedure returns the never-smeared spectrum. Finally, we display the effect of the corrections for the acceptance and resolution smearing in Figure 6.12. As expected, the acceptance correction affects the normalization, while the unsmearing affects the shape. 
Columns of the C Matrix

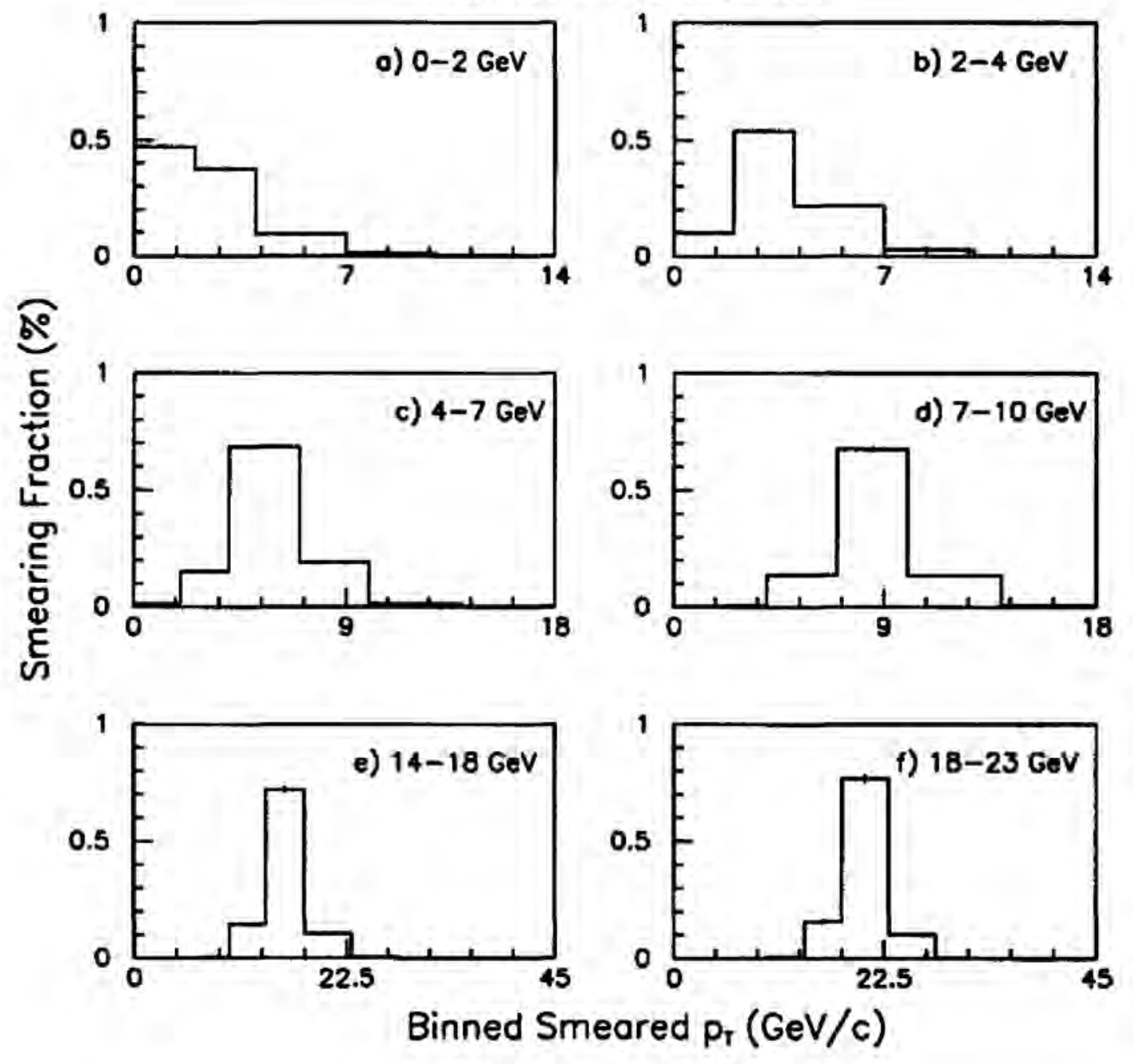

Figure 6.9: The "smearing fraction", or the probability that the $p_{T}$ in a given range is measured to be in some other range because of resolution smearing. 

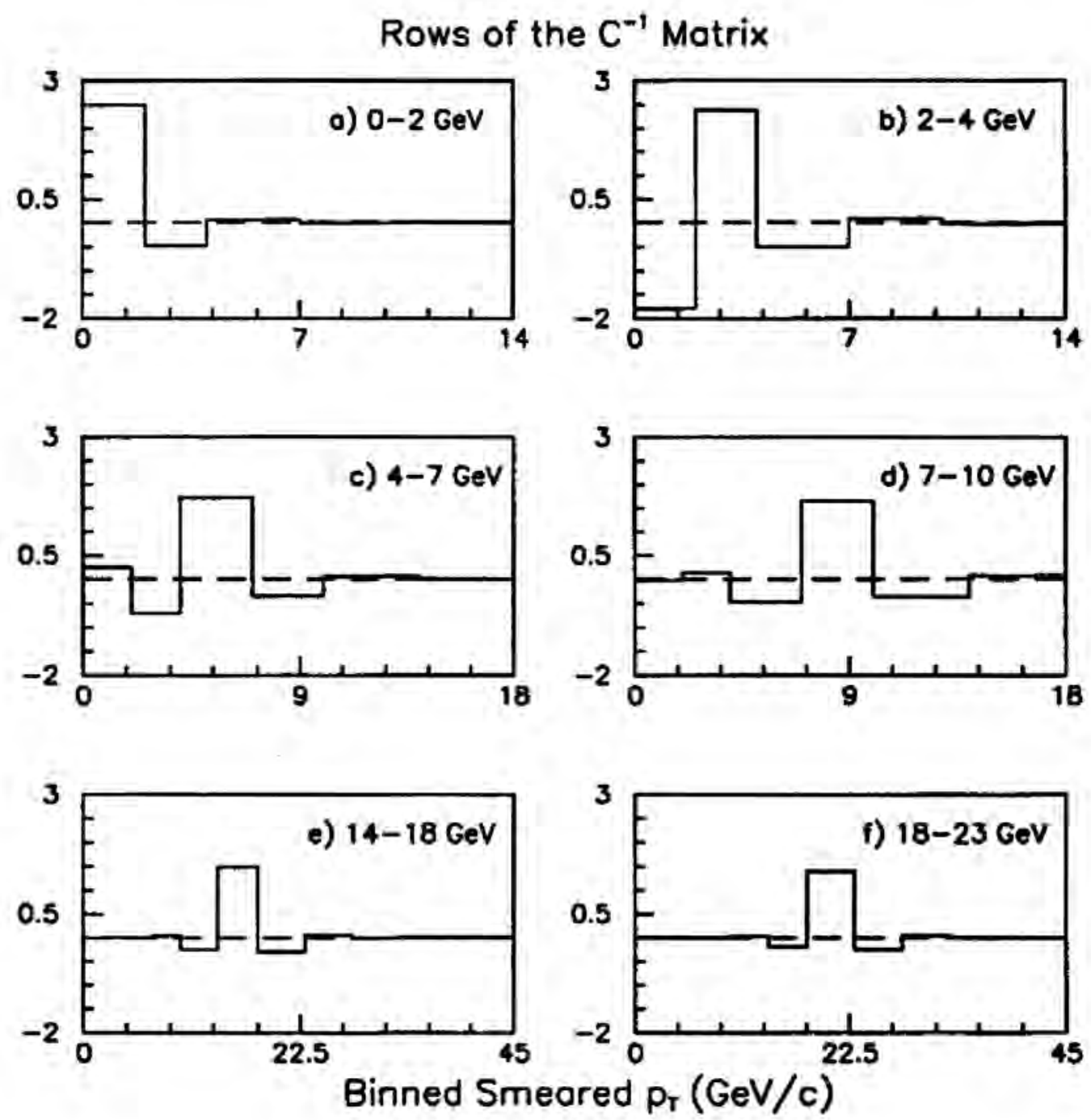

Figure 6.10: The "unsmearing" mechanism. It shows how the content in each observed bin is to be distributed among the "unsmeared" bins. The dashed line is at zero. 
Table 6.1: The smearing matrix $C_{i j}$. For example, the matrix element $C_{32}$ is 0.22 .

\begin{tabular}{r|rrrrrrrrrrrrr}
\hline$i \backslash j$ & 1 & 2 & 3 & 4 & 5 & 6 & 7 & 8 & 9 & 10 & 11 & 12 & 13 \\
\hline 1 & 0.47 & 0.10 & 0.01 & 0 & 0 & 0 & 0 & 0 & 0 & 0 & 0 & 0 & 0 \\
2 & 0.37 & 0.54 & 0.15 & 0.01 & 0 & 0 & 0 & 0 & 0 & 0 & 0 & 0 & 0 \\
3 & 0.09 & 0.22 & 0.68 & 0.14 & 0.01 & 0 & 0 & 0 & 0 & 0 & 0 & 0 & 0 \\
4 & 0.01 & 0.03 & 0.19 & 0.67 & 0.16 & 0 & 0 & 0 & 0 & 0 & 0 & 0 & 0 \\
5 & 0 & 0 & 0.01 & 0.13 & 0.74 & 0.15 & 0.01 & 0 & 0 & 0 & 0 & 0 & 0 \\
6 & 0 & 0 & 0 & 0 & 0.12 & 0.72 & 0.16 & 0.01 & 0 & 0 & 0 & 0 & 0 \\
7 & 0 & 0 & 0 & 0 & 0 & 0.10 & 0.77 & 0.13 & 0 & 0 & 0 & 0 & 0 \\
8 & 0 & 0 & 0 & 0 & 0 & 0 & 0.10 & 0.74 & 0.14 & 0.01 & 0 & 0 & 0 \\
9 & 0 & 0 & 0 & 0 & 0 & 0 & 0 & 0.12 & 0.72 & 0.20 & 0 & 0 & 0 \\
10 & 0 & 0 & 0 & 0 & 0 & 0 & 0 & 0 & 0.06 & 0.86 & 0.10 & 0 & 0 \\
11 & 0 & 0 & 0 & 0 & 0 & 0 & 0 & 0 & 0 & 0.03 & 0.90 & 0.08 & 0 \\
12 & 0 & 0 & 0 & 0 & 0 & 0 & 0 & 0 & 0 & 0 & 0.03 & 0.89 & 0.22 \\
13 & 0 & 0 & 0 & 0 & 0 & 0 & 0 & 0 & 0 & 0 & 0 & 0.01 & 0.93 \\
\hline
\end{tabular}
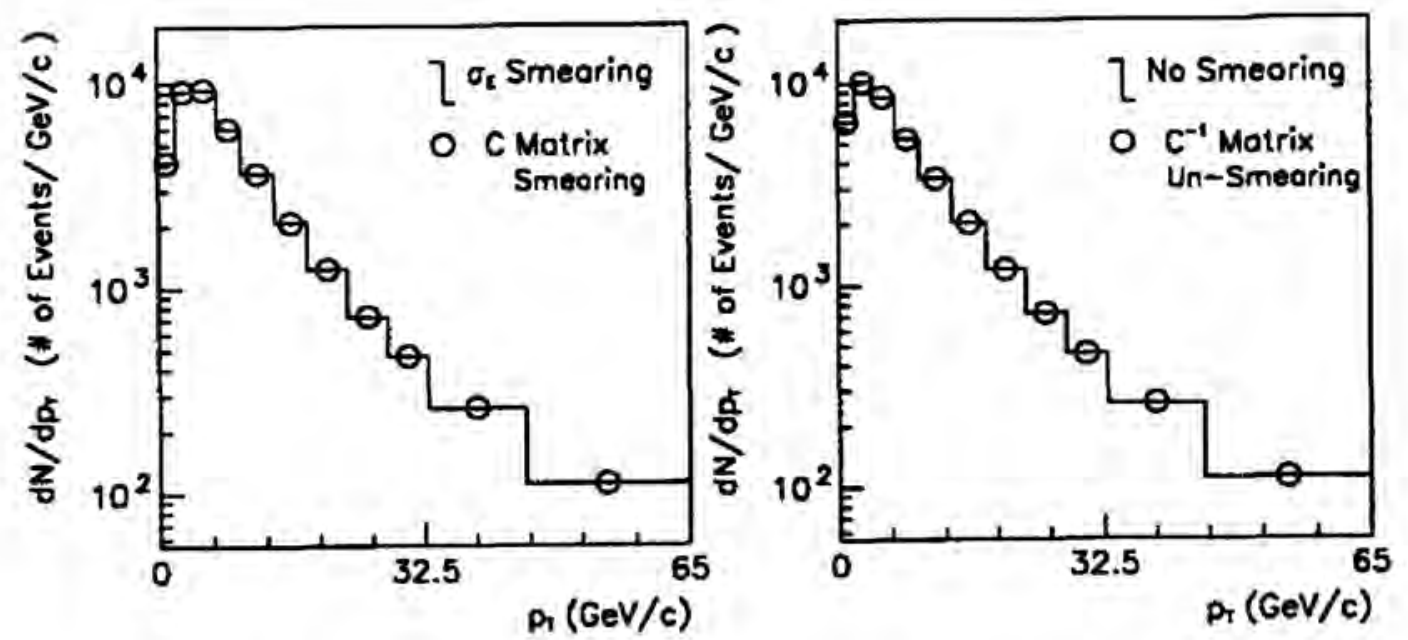

Figure 6.11: The results of consistency tests of the smearing and "unsmearing" matrices. 
Corrections to $p_{\text {r Spectrum }}$
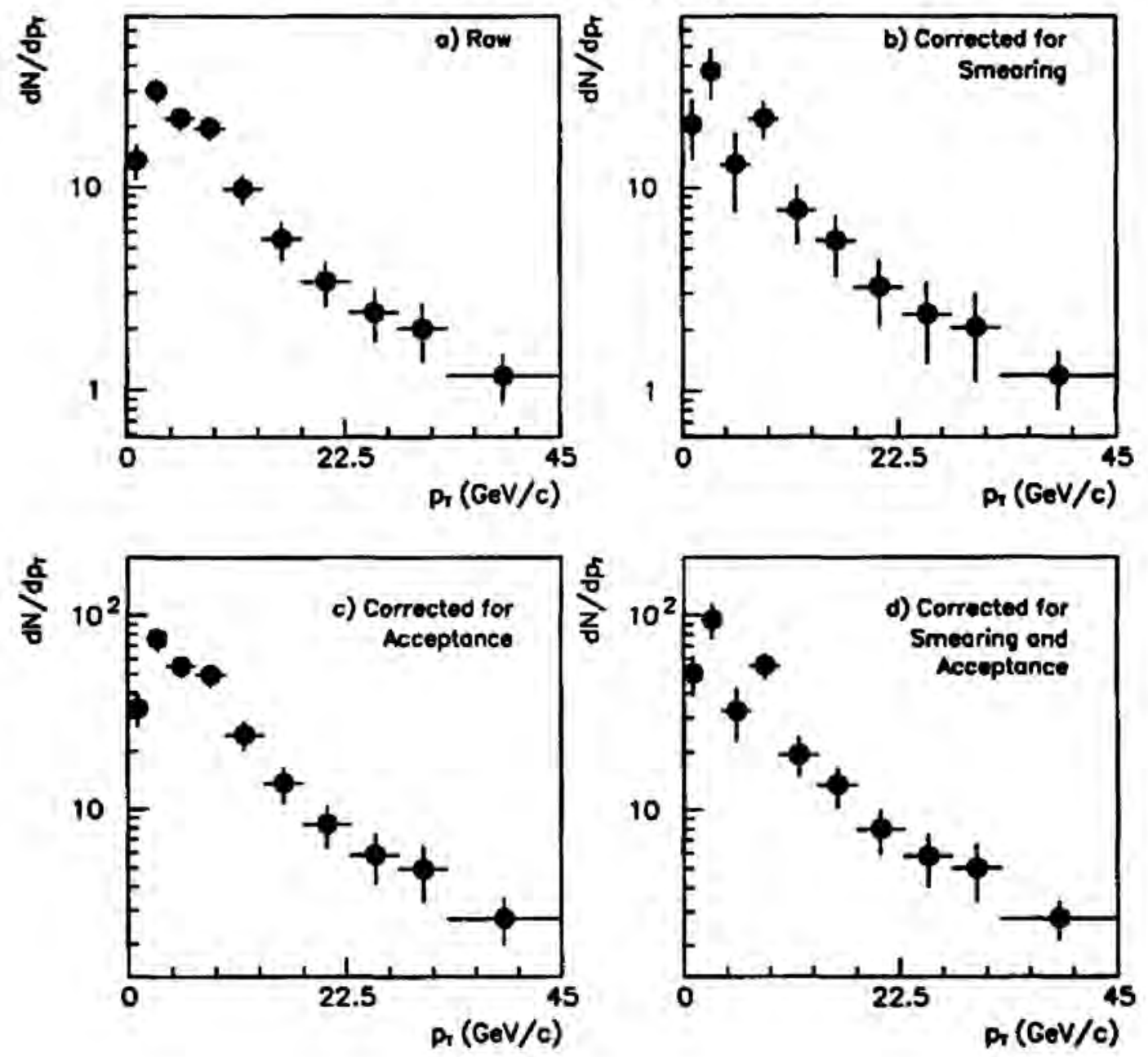

Figure 6.12: The effects of corrections on the $p_{T}$ spectrum. Note that the correction for resolution smearing changes the shape, whereas the correction for acceptance changes the over-all normalization of the spectrum. 


\subsection{Background Estimate}

As shown in Figure 4.13, non-dilepton background contamination in our $Z$ sample is very small. Fake muon background comes from pion punch-through, Fake electron background comes from mis-identified jets. Backgrounds in our $Z$ sample can be estimated using the isolation of events in the side band regions to extrapolate into the signal region. The scatter plots used to estimate the background in the electron and muon samples are shown in Figure 6.13. The maximum of the two lepton isolations is used in the plot.

Note that the regions "a" plus "c", "b", "d" plus "e", and " $\mathrm{Z}$ " in Figure 6.13 all have the same area. For the $Z \rightarrow$ pe sample, we estimate the background in the signal region, labeled " $\mathrm{Z}$ ", using three methods:

1. Use the number of events in the side band regions "a" and " $c$ ". There are 2 events each in "a" and "c". Assuming the background falls linearly in mass, an interpolation gives $2 \pm 2$ background events in the " $b$ " region. Then, assuming conservatively (see next item) that the background is flat in maximum isolation as the maximum isolation tends to zero, an extrapolation gives $2 \pm 2$ background events in the "Z" region.

2. Use the number of events in the region "b". This region contains 12 events - these consist of both background events and real $Z$ events that failed the isolation cut, Using the CEM isolation cut efficiency and the total number of $Z$ events, we estimate $\left(1-0.985^{2}\right) \times 235=7$ real $Z$ events, leaving $5 \pm 3$ background events in the " $b$ " region.

But the distribution of the maximum isolation variable is not flat as it tends to zero. Since it is the maximum of two quantities that range from zero upward, the probability that it is zero is much less than the probability that it is non-zero. The distributions of the maximum isolation in the background processes: a)photon conversion, [54] b) $\pi^{0} \mathrm{~s}$ in jets,[55] and c) $b \vec{b}$ semi-leptonic decays [55] are shown in Figure 6.14. These distributions suggest that the background falls linearly to zero as we extrapolate from the region " $\mathrm{b}$ " to the signal region " $\mathrm{Z}$ ". We thus estimate $1 \pm 1$ and $3 \pm 2$ background 
events using method 1 and method 2 , respectively.

3. Use the number of events in the side band regions " $d$ " and " $e$ ". There are 7 events in " $\mathrm{d}$ " and 3 events in " $\mathrm{e}$ ". Using the ISAJET Monte Carlo with detector simulation (see Section 6.1), we expect the Drell-Yan and $Z$ processes to contribute, for 235 events in the " $Z$ " region, 6 events in " $d$ " and 4 events in " $e$ ". Thus, we estimate $1 \pm 1$ background event in " $\mathrm{Z}$ ".

We conclude that the background from QCD jet production is $2 \pm 2$ events. This is less than $1 \%$ of the events in the $Z \rightarrow$ ee sample.

For the $Z \rightarrow \mu \mu$ sample, we cannot use the maximum isolation variable to estimate the background because we did not use isolation to define our signal region. (That is, muon isolation cut is not used in muon selection.) But there is only one event in all of the side band regions (in "d"). We estimate conservatively that the background in the $Z \rightarrow \mu \mu$ sample is $1 \pm 1$ event, or $1 \%$ of the sample.

The background from $Z \rightarrow \tau \tau$, with sequential $\tau$ decays to electrons or muons, is estimated using the ISAJET Monte Carlo to be less than 0.5 event.

In summary, the backgrounds in both the $Z \rightarrow e e$ and $Z \rightarrow \mu \mu$ samples are less than $1 \%$. We neglect these backgrounds.

\subsection{Correction for the Drell-Yan Continuum}

Since we compare our measurement with theoretical calculations that include the $Z$ couplings only; we must correct for contributions from the virtual photon. We obtain the correction as the ratio:

$$
f_{\mathrm{DY}}=\frac{\sigma_{Z}}{\sigma_{Z+Z \cdot \gamma+\gamma}}
$$

where

$$
\sigma_{Z}=\int_{75 G e V}^{105 G e V} \frac{d \sigma(Z)}{d m} \cdot d m
$$




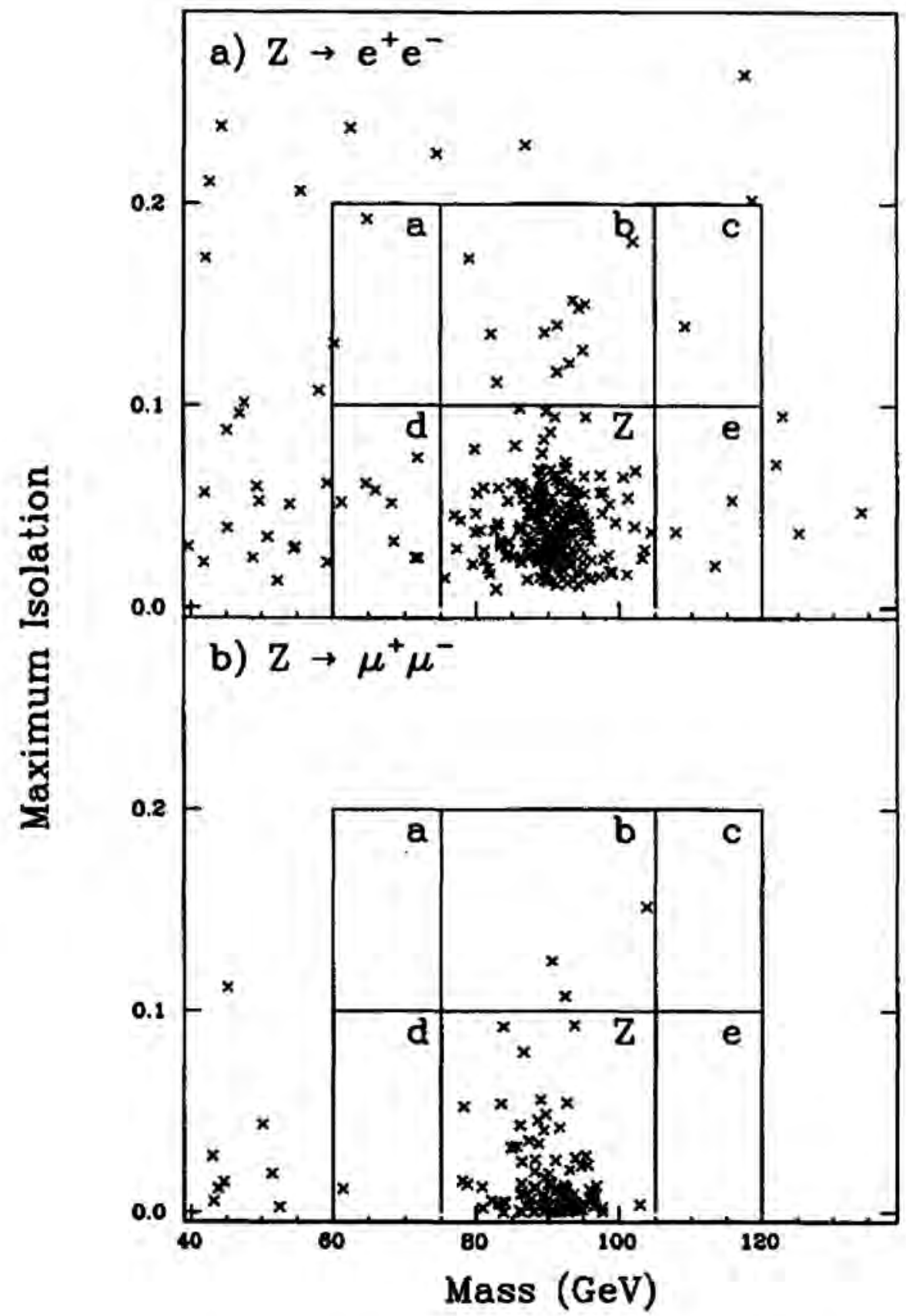

Figure 6.13: Scatter plot of the maximum isolation of the two charged leptons vs. the invariant mass used in background estimate. 


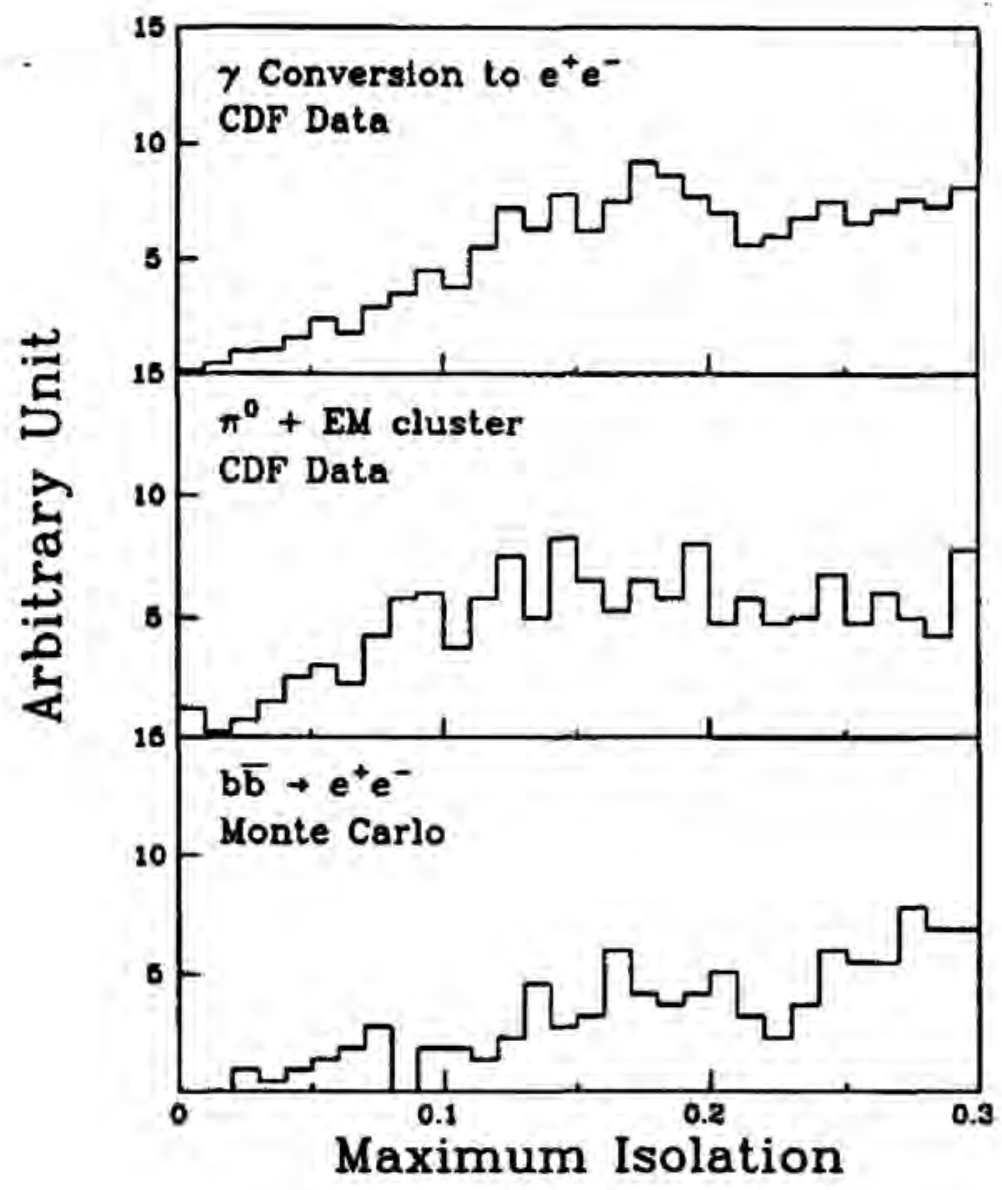

Figure 6.14: The distributions of maximum isolation for three background processes. 
is the cross section in the mass window $75-105 \mathrm{GeV}$ with $Z$ couplings only, and

$$
\sigma_{Z+Z \cdot \gamma+\gamma}=\int_{75 G e V}^{105 G e V} \frac{d \sigma(Z+Z \cdot \gamma+\gamma)}{d m} \cdot d m
$$

is the cross section with contributions from the $Z$, the $\gamma$, and the $Z-\gamma$ interference terms. The cross section calculation is done using the leading order results. We obtain $f_{\mathrm{DY}}=\mathbf{0 . 9 8 7}$ $\pm 0.005 .[32]$ We apply this correction to each $p_{T}$ bin. 


\section{Chapter 7}

\section{Results and Conclusions}

With the correction for acceptance obtained in Section 6.2 and the correction for resolution smearing obtained in Section 6.3, we now measure the $Z$ production differential cross section as a function of $p_{T}, d \sigma / d p_{T}$, and compare it directly with theoretical predictions. In this Chapter, we describe the procedure for obtaining $d \sigma / d p_{T}$ and the uncertainty in the measurement, along with the results and conclusions.

\subsection{The Procedure for Obtaining $d \sigma / d p_{T}$}

We apply corrections for acceptance and resolution smearing as follows:

1. Correct for resolution smearing effects using $C_{i j}^{-1}$, as in Equation 6.10 :

$$
F_{j}=\sum_{i} C_{i j}^{-1} \cdot F_{i}^{\prime}
$$

2. Correct for the acceptance.

3. Correct for the Drell-Yan continuum contribution and the leptonic branching fraction.

The effect of step 1 is shown in Figure 6.12b. The effect of step 2 is shown in Figure 6.12c. The differential cross-section as a function of $p_{T}$ is then given by:

$$
d \sigma / d p_{T}=\frac{N\left(p_{T}\right) \cdot f_{\mathrm{DY}}}{a\left(p_{T}\right) \cdot \Delta p_{T} \cdot \mathcal{L} \cdot B}
$$

where 
- $N\left(p_{T}\right)$ is the number of events in a $p_{T}$ bin with bin center $p_{T}$ and width $\Delta p_{T}$, after correction for resolution smearing. That is, $N_{j}=F_{j} \cdot \Delta p_{T}$.

- $a\left(p_{T}\right)$ is the combined $Z \rightarrow e e$ and $Z \rightarrow \mu \mu$ acceptance defined in Equation 6.3.

- $\mathcal{L}=(4.05 \pm 0.28) \mathrm{pb}^{-1}$ is the integrated luminosity.

- $B=0.033$ is the $Z \rightarrow e e, Z \rightarrow \mu \mu$ branching fraction.

- $f_{\mathrm{DY}}=0.987 \pm 0.005$ is the correction for Drell-Yan continuum contribution to the $Z$ peak.

Note that we use the integrated luminosity of the electron sample for this measurement. The acceptance for the combined $Z \rightarrow e e$ and $Z \rightarrow \mu \mu$ sample accounts for the smaller effective integrated luminosity of the muon sample, $\mathcal{L}=(3.54 \pm 0.24) \mathrm{pb}^{-1}$. (See Section 6.2.)

\subsection{The Statistical Uncertainty in $d \sigma / d p_{T}$}

The sources of uncertainties in our measurement include statistical fluctuations in the number of events observed in a raw $\boldsymbol{p}_{T}$ bin, assumptions made in the Monte Carlo simulation used to obtain the corrections for acceptance and resolution smearing, and the uncertainties on other normalization factors in Equation 7.2 such as the integrated luminosity. Because the total number of events must be the same before and after "unsmearing", any procedure used to correct resolution smearing effects leads to correlation in the uncertainties among the bins in the unsmeared $p_{T}$ spectrum. We first consider the correlation in the statistical uncertainty. Let $N_{k}^{\prime}\left(p_{T i}^{\prime}\right)$ be the number of events observed in the $i^{\text {th }} p_{T}$ bin. The statistical uncertainty on the observed spectrum is given by the Poisson fluctuation in the number of events observed in each bin:

$$
\Delta F_{i}^{\prime}=\frac{\Delta N_{i}^{\prime}}{\Delta p_{T}}=\frac{\sqrt{N_{i}^{\prime}}}{\Delta p_{T}} .
$$

The statistical uncertainty on the unsmeared spectrum is

$$
\Delta F_{j}=\sum_{i} C_{i j}^{-1} \cdot \Delta F_{i}^{\prime}
$$


and the covariance is

$$
\left(\Delta F_{i}\right)\left(\Delta F_{j}\right)=\sum_{k T} C_{k i}^{-1} \Delta F_{k}^{\prime} C_{l j}^{-1} \Delta F_{i}^{\prime} .
$$

By taking the expectation value, and using the fact the Poisson fluctuations in each bin of the observed spectrum are independent, we obtain the covariance matrix for the "unsmeared" spectrum:

$$
\begin{aligned}
E_{i j} & =\left\langle\left(\Delta F_{i}\right)\left(\Delta F_{j}\right)\right\rangle \\
& =\left\langle\sum_{k T} C_{k i}^{-1} \Delta F_{k}^{\prime} C_{l j}^{-1} \Delta F_{i}\right\rangle \\
& =\sum_{k I} C_{k i}^{-1} C_{l j}^{-1}\left\langle\left(\Delta F_{k}^{\prime}\right)\left(\Delta F_{i}^{\prime}\right)\right\rangle \\
& =\sum_{k I}^{k} C_{k i}^{-1} C_{l j}^{-1}\left(\Delta F_{k}^{\prime}\right)^{2} \delta_{k l} \\
& =\sum_{k} C_{k i}^{-1} C_{k j}^{-1}\left(\Delta F_{k}^{\prime}\right)^{2} .
\end{aligned}
$$

The square-root of the diagonal elements of $E_{i j}$ gives the error bars shown in Figure 6.12d. Similarly, as we can see from Equation 7.1, the uncertainties in the unsmearing matrix $C_{i j}^{-1}$ due to systematic uncertainties, also lead to correlated uncertainties among the bins of the $p_{T}$ spectrum. We next discuss the sources of systematic uncertainties, and then the treatment of the correlated uncertainties.

\subsection{The Sources Of Systematic Uncertainties}

The sources that contribute systematic uncertainties are

- the integrated luminosity: $\mathcal{L}=(4.05 \pm 0.28) \mathrm{pb}^{-1}$,

- the Drell-Yan continuum correction: $f_{\mathrm{DY}}=0.987 \pm 0.005$, and

- the assumptions in the Monte Carlo simulation.

The following is a list of assumptions made in the Monte Carlo simulation, described in Section 6.1, that lead to uncertainties in the corrections for acceptance and resolution smearing. We discuss how to treat correlated uncertainties in Section 7.4. 


\section{The Event Vertex}

The event $z$-vertex distribution is a Gaussian, with $\sigma=30 \pm 1 \mathrm{~cm}$. For a $z$-vertex cut at $60 \mathrm{~cm}$ from the center of the detector, this corresponds to an efficiency of $\epsilon_{z v}=0.954 \pm 0.005$.

\section{Structure Functions}

Several sets of the parametrizations of the parton momentum distributions, MRS sets E and B, and DFLM sets 1,2 and 3 are used in the Monte Carlo event generation. Since a variation in geometric acceptance means that the fraction of events in each detector changes, it is correlated with the $Z$ selection efficiency. This correlation is taken into account in the acceptance calculation described in Section 6.2. The nominal set used is MRS set $B$. The resulting maximum deviation in $d \sigma / d p_{T}$ from the nominal case is used as the one standard deviation uncertainty in $d \sigma / d p_{T}$ due to the choice of structure function.

\section{Selection and Trigger Efficiencies}

The lepton selection and trigger efficiencies for each lepton type are varied independently within their uncertainties. The resulting variances in the $d \sigma / d p_{T}$ results are added. (This is just combining errors in quadrature.)

\section{Energy and Momentum Scales}

The energy and momentum scale factors are set to 1.0 in the Monte Carlo. We estimate the uncertainty by varying the scale factors, as described in Section 6.1.2. The resulting variances in the $d \sigma / d p_{T}$ results are added.

\section{Energy and Momentum Resolution Parametrizations}

The amount of resolution smearing in the Monte Carlo simulation affects the resolution smearing correction. As discussed in Section 6.1.2, we independently vary each term in the momentum resolution parametrizations within its uncertainties. For each variation, a smearing matrix $C_{i j}$, and thus its inverse $C_{i j}^{-1}$, is obtained to estimate 
the systematic uncertainty. (See Section 7.4.)

It affects the selection efficiency as well, through smearing in the electron $E_{T}$ threshold and the mass window cut. But the effect on the selection efficiency is very small and we ignore this correlation between resolntion correction and selection efficiency.

\section{Monte Carlo $p_{T}$ Spectrum}

The shape of the $p_{T}$ spectrum affects the resolution smearing correction, because the amount of smearing depends on both the resolution, and the shape of the $p_{T}$ spectrum inside each bin. As discussed in Section 6.1.3, the Monte Carlo $p_{T}$ spectrum is adjusted to fit the data. The uncertainties in the fit parameters are used to estimate the systematic uncertainty. That is, we obtain a smearing matrix $C_{i j}$, and thus its inverse $C_{i j}^{-1}$, for each input Monte Carlo $p_{T}$ spectrum obtajned from varying the fit parameters. (See Section 7.4.)

The uncertainties from statistical fluctuations and uncertainties in the "unsmearing" mar trix lead to uncertainties correlated among the bins, resulting in a covariance matrix. The uncorrelated variances due to the sources listed above, that is, those not from the resolution and the input Monte Carlo $p_{T}$ spectrum, are added to the diagonal of the covariance matrix. The uncorrelated uncertainty on $d \sigma / d p_{T}$ due to each source, as well as the diagonal elements of the covariance matrix of the correlated uncertainties are summarized separately in Table 7.1 in Section 7.5. We next describe the procedure for obtaining the covariance matrix.

\subsection{The Treatment of Correlated Uncertainties}

To account for correlation in the uncertainties, we use a simple Monte Carlo procedure that models the results of a large number of experiments to obtain a covariance matrix. Here is the outline of this procedure:

1. Generate a raw $p_{T}$ distribution, allowing for statistical fluctuations. 
2. Generate an "unsmearing" matrix, allowing for systematic uncertainties in the resolition and Monte Carlo $p_{T}$ spectrum.

3. Apply the generated "unsmearing" matrix to the generated raw $p_{T}$ distribution.

4. Get $d \sigma / d p_{T}$ as in Equation 7.2.

5. The deviation of this $d \sigma / d p_{T}$ from the nominal $d \sigma / d p_{T}$ measured from real data is used to obtain the covariance matrix.

More discussions of this procedure follow. It folds in the systematic uncertainties and the statistical uncertainties that are correlated among bins.

\subsubsection{Statistical Uncertainties Only}

We first consider the simple case with statistical nncertainty only. A $p_{T}$ spectrum is generated according to raw data. For each $p_{T}$ bin, a random deviate is drawn from a Poisson distribution. The mean of this Poisson distribution is given by the number of events in the corresponding raw data bin. For each raw $p_{T}$ spectrum thus generated, we apply the corrections for acceptance and resolution smearing as done for real data. This results in a $d \sigma / d p_{T}$ that we expect to measure if we were to repeat our experiment, assuming only statistical fluctuations in the data. We repeat this process $N$ times to obtain a covariance matrix:

$$
H_{i j}=\frac{1}{N} \cdot \sum_{k=1}^{N}\left(X_{i}^{k}-\bar{X}_{i}\right) \cdot\left(X_{j}^{k}-\bar{X}_{j}\right)
$$

where $N=10^{6}, X_{i}^{k}$ is the $i^{\text {th }}$ bin of $d \sigma / d p_{T}$ in the $k^{\text {th }}$ Monte Carlo experiment, and $\bar{X}_{i}$ is the nominal value given by the real data. The statistical fluctuations in $d \sigma / d p_{T}$ given by $H_{i j}$ is the same as that in $F$ given by $E_{i j}$ which is obtained analytically in Equation 7.6. (That is, $H_{i j}$ is the same as $E_{i j}$ when the other factors in Equation 7.2 are accounted for.) This validates our simple Monte Carlo procedure. 


\subsubsection{Including Systematic Uncertainties}

We now consider correlated systematic uncertainties. We assume that systematic uncertainties are Gaussian. Ideally, for each Monte Carlo experiment, we would allow each parameter in the resolution parametrization and the Monte Carlo $p_{T}$ spectrum to vary independently, and obtain $C_{i j}^{-1}$ as described in Section 6.3. However, this is very inefficient. Obtaining $C_{i j}^{-1}$ requires analyzing a large sample of Monte Carlo $Z$ events. Besides, the dominant correlated uncertainties are statistical. Thus, we generate $C_{i j}^{-1}$, allowing for systematic uncertainties, as follows:

1. For each parameter to be varied in the resolution parametrization and the Monte Carlo $p_{T}$ spectrum, evaluate $C_{i j}$ only three times: once at the nominal parameter value, and once each at the $\pm 1 \sigma$ value. These are the nominal $C_{i j}$ matrix, and the $\pm 1 \sigma$ matrices that would give the $\pm 1 \sigma$ deviations on $d \sigma / d p_{T}$ due to the uncertainty on this particular parameter.

This step is done only once. The following steps are done once for each "experiment" in our Monte Carlo procedure.

2. A new $C_{i j}$ matrix is generated by varying the nominal $C_{i j}$ matrix. The sign and the amount of the variation is given by the value of the $\pm 1 \sigma$ element with respect to the nominal matrix element value. The variations are scaled by a random number drawn from a Gaussian with mean $=0$ and $\sigma=1$. The variations from either the + or the $-1 \sigma$ matrix are used, according to the sign of the random number. The scaled variation for each element of the smearing matrix is added to (or subtracted from) the corresponding element of the nominal $C_{i j}$ matrix. For example, suppose for the $(1,1)$ element, the nominal smearing matrix is 0.60 and the $+1 \sigma$ value is 0.65 . Then, the $+1 \sigma$ value is generated by fluctuating 0.60 up by a Gaussian of $\sigma=0.05$. The generated smearing matrix retains the correlation between the variations in each element of the smearing matrix due to variation of the parameter. This process amounts to a Taylor 
expansion of the nominal $C_{i j}$ matrix, which is a valid procedure since the variations are small.

3. The final $C_{i j}$ is derived by varying each element consecutively for all parameter variations. That is, the value obtained by fluctuating the nominal value due to parameter 1 is further fluctuated by variations due to parameter 2 , and so on. This amounts to adding Gaussian errors in quadrature.

4. The generated $C_{i j}$ is normalized, as for the real $C_{i j}$, so that the total number of events is the same before and after smearing. And finally, it is inverted to yield a generated $C_{i j}^{-1}$.

The only modification to the procedure described for the case with statistical uncertainty

only is the generation of a new $C_{i j}^{-1}$ for each Monte Carlo experiment. This is the process outlined at the beginning of this Section. We thus obtain a covariance matrix, $H_{i j}$, as defined in Equation 7.7, that describes the uncertainties that are correlated among the bins and includes both the statistical fluctuations and the systematic uncertainty in the "unsmearing" matrix.

\subsection{Results of the $Z p_{T}$ Distribution}

We add the uncorrelated variances in $d \sigma / d p_{T}$ to the diagonal of $H_{i j}$, to get the final covariance matrix $\sigma_{i j}$. This amounts to combining variances, or adding errors in quadrature. Note that $\sigma_{i}=\sqrt{\sigma_{i i}}$ is the one standard deviation uncertainty on the $i^{\text {th }}$ bin of the measured $d \sigma / d p_{T}$. Using this covariance matrix, we obtain a $\chi^{2}$ when making comparisons with theoretical predictions. The correlation coefficient between neighboring bins is given by

$$
\rho_{i j}=\frac{\sigma_{i j}}{\sqrt{\sigma_{i i} \cdot \sigma_{j j}}}
$$

The one standard deviation uncertainties due to each source are listed in Table 7.1. For the uncertainties that are correlated among the bins, the values of the square-root of the 
Table 7.1: The percentage uncertainties broken down for each source. In the case of correlated errors, the diagonals of the covariance matrix are used.

\begin{tabular}{|c|c|c|c|c|c|c|c|}
\hline Bin \# & $\begin{array}{c}\text { Statistical } \\
\text { Fluctuation }\end{array}$ & $\begin{array}{l}\text { Structure } \\
\text { Function }\end{array}$ & $\begin{array}{l}e, \mu \\
\in I D\end{array}$ & $\begin{array}{c}e, \mu \\
\epsilon \text { Thigger }\end{array}$ & $\begin{array}{c}\text { E,p } \\
\text { Scale }\end{array}$ & $\begin{array}{c}\mathbf{E , p} \\
\text { Resolution }\end{array}$ & $\begin{array}{l}\text { Input } p_{T} \\
\text { Spectrum }\end{array}$ \\
\hline$\overline{1}$ & $33 \%$ & $6 \%$ & $5 \%$ & $1 \%$ & $13 \%$ & $7 \%$ & $4 \%$ \\
\hline 2 & $28 \%$ & $5 \%$ & $5 \%$ & $1 \%$ & $8 \%$ & $5 \%$ & $4 \%$ \\
\hline 3 & $41 \%$ & $8 \%$ & $7 \%$ & $2 \%$ & $21 \%$ & $16 \%$ & $15 \%$ \\
\hline 4 & $20 \%$ & $3 \%$ & $6 \%$ & $1 \%$ & $4 \%$ & $5 \%$ & $4 \%$ \\
\hline 5 & $31 \%$ & $3 \%$ & $6 \%$ & $1 \%$ & $9 \%$ & $6 \%$ & $5 \%$ \\
\hline 6 & $33 \%$ & $5 \%$ & $6 \%$ & $1 \%$ & $3 \%$ & $5 \%$ & $2 \%$ \\
\hline 7 & $36 \%$ & $4 \%$ & $6 \%$ & $1 \%$ & $4 \%$ & $3 \%$ & $3 \%$ \\
\hline 8 & $42 \%$ & $8 \%$ & $6 \%$ & $2 \%$ & $5 \%$ & $5 \%$ & $2 \%$ \\
\hline 9 & $45 \%$ & $7 \%$ & $6 \%$ & $1 \%$ & $5 \%$ & $6 \%$ & $3 \%$ \\
\hline 10 & $32 \%$ & $3 \%$ & $6 \%$ & $1 \%$ & $2 \%$ & $2 \%$ & $2 \%$ \\
\hline 11 & $49 \%$ & $5 \%$ & $6 \%$ & $2 \%$ & $4 \%$ & $4 \%$ & $2 \%$ \\
\hline 12 & $53 \%$ & $5 \%$ & $6 \%$ & $1 \%$ & $5 \%$ & $2 \%$ & $3 \%$ \\
\hline 13 & $66 \%$ & $13 \%$ & $5 \%$ & $1 \%$ & $11 \%$ & $4 \%$ & $4 \%$ \\
\hline
\end{tabular}

diagonal elements of the covariance matrix are given. The full correlation matrix defined in Equation 7.8 is presented in Table 7.2 .

We summarize the results of the $Z p_{T}$ distribution and the uncertainties in Table 7.3. The results are obtained with a combined sample of $235 Z \rightarrow$ ee and $103 Z \rightarrow \mu \mu$ events. The uncertajnties are systematic and statistical combined, and are correlated among bins due to correction for resolution smearing. The dominant elements of the correlation matrix are also included.

The $p_{T}$ values are corrected for binning effects such that in the limit of infinite statistics, the plotted points lie on the true spectrum.[56] This is accomplished using the following procedure:

1. Start with a functional parametrization, $f\left(p_{T}\right)$ that fits the measured $d \sigma / d p_{T}$ with the $p_{T}$ values at the center of the bin. (See below for more details on the parametrization.)

2. Then for a bin with edges at $x_{1}$ and $x_{2}$, the proper position to plot the data point, 
Table 7.2: The correlation matrix. (It is symmetric about the diagonal.)

\begin{tabular}{|c|c|c|c|c|c|c|c|c|c|c|c|c|c|}
\hline & 1 & $\overline{2}$ & 3 & $\overline{4}$ & 5 & 6 & 7 & 8 & 9 & 10 & 11 & 12 & 13 \\
\hline 1 & 1 & -0.45 & 0.07 & -0.02 & -0.02 & 0.03 & 0.01 & -0.02 & 0.01 & $\overline{0}$ & -0.01 & .0 .01 & -0.1 \\
\hline 2 & & 1 & -0.48 & 0.22 & -0.10 & 0.04 & 0.02 & -0.02 & 0.01 & -0.01 & -0.01 & 0 & -0.01 \\
\hline 3 & & & 1 & -0.50 & 0.23 & -0.10 & -0.03 & 0,05 & -0.03 & 0.01 & 0.03 & 0.01 & 0.03 \\
\hline 4 & & & & 1 & -0.43 & 0.14 & 0.01 & -0.03 & 0.02 & -0.01 & -0.02 & -0.01 & -0.02 \\
\hline 5 & & & & & 1 & -0.38 & 0.07 & 0 & -0.01 & 0.02 & 0.01 & 0.01 & 0.01 \\
\hline 6 & & & & & & 1 & -0.33 & 0.06 & -0.01 & 0 & -0.01 & 0 & 0 \\
\hline 7 & & & & & & & 1 & -0.29 & 0.08 & 0 & 0 & 0 & -0.01 \\
\hline 8 & & & & & & & & 1 & -0.37 & 0.04 & 0.01 & 0 & 0 \\
\hline 9 & & & & & & & & & 1 & -0.18 & 0.02 & 0 & 0.01 \\
\hline 10 & & & & & & & & & & 1 & -0.16 & 0.02 & 0 \\
\hline 11 & & & & & & & & & & & 1 & -0.17 & 0.02 \\
\hline 12 & & & & & & & & & & & & 1 & -0.10 \\
\hline 13 & & & & & & & & & & & & & 1 \\
\hline
\end{tabular}

$x_{0}$ is given by solving:

$$
\int_{x_{1}}^{x_{2}} f(x) d x=f\left(x_{0}\right) \cdot\left(x_{2}-x_{1}\right)
$$

3. Using the new bin position in step 1 , repeat the last two steps. Only one iteration is needed to arrive at the final bin position for each bin.

4. The plotted point is at $p_{T}=x_{0}$, with the vertical-axis value given by the measured $d \sigma / d p_{T}$ in that bin.

Then, for the simple case of a linear spectrum, $x_{0}=\left(x_{2}+x_{1}\right) / 2$, as expected. Note that taking the average, with or without weighting by the spectrum, of the $p_{T}$ inside the bin would give the wrong position. That is, the plotted points would not lie on the true spectrum. This is easily seen for the example of a linear spectrum.

The functional form used for the parametrization in this procedure, which was also used for sculpting in Section 6.1.3, is a modified version of the function used in the ISAJET Monte Carlo to generate the boson $p_{T}$ distributions:

$$
\frac{d \sigma}{d p_{T}}=\frac{2 p_{T}\left(p_{T}^{2}-D\right)^{C-1}}{B\left[\left(E p_{T}\right)^{F}+1\right]}
$$


Table 7.3: Summary of the results and uncertainties.

\begin{tabular}{ccccccccc}
\hline \hline $\begin{array}{c}\Delta p_{T} \\
(\mathrm{GeV} / c)\end{array}$ & $\begin{array}{c}\text { Events } \\
\text { Observed }\end{array}$ & $\begin{array}{c}p_{T} \\
(\mathrm{GeV} / \mathrm{c})\end{array}$ & \multicolumn{2}{c}{$d \sigma / d p_{T}$} & \multicolumn{3}{c}{ Correlation Coefficients } \\
\cline { 6 - 9 } & $(\mathrm{pb} / \mathrm{GeV} / c)$ & $(i, i+1)$ & $(i, i+2)$ & $(i, i+3)$ \\
\hline $0-2$ & 27 & 1.0 & 391 & \pm & 139 & -0.45 & 0.07 & -0.02 \\
$2-4$ & 60 & 2.8 & 734 & \pm & 214 & -0.48 & 0.22 & -0.10 \\
$4-7$ & 66 & 5.6 & 251 & \pm & 144 & -0.50 & 0.23 & -0.10 \\
$7-10$ & 59 & 8.4 & 427 & \pm & 110 & -0.43 & 0.14 & 0.01 \\
$10-14$ & 39 & 12.0 & 149 & \pm & 55 & -0.38 & 0.07 & 0.00 \\
$14-18$ & 22 & 16.0 & 104 & \pm & 38 & -0.32 & 0.06 & -0.01 \\
$18-23$ & 17 & 20.3 & 61.5 & \pm & 23.5 & -0.29 & 0.08 & 0.00 \\
$23-28$ & 12 & 25.3 & 44.6 & \pm & 20.4 & -0.35 & 0.04 & 0.01 \\
$28-33$ & 10 & 30.4 & 38.7 & \pm & 17.6 & -0.18 & 0.02 & 0.00 \\
$33-45$ & 14 & 38.4 & 21.5 & \pm & 7.0 & -0.15 & 0.02 & 0.00 \\
$45-65$ & 6 & 54.0 & 4.7 & \pm & 2.5 & -0.17 & 0.02 & - \\
$65-90$ & 4 & 76.3 & 2.8 & \pm & 1.6 & -0.10 & - & - \\
$90-180$ & 2 & 126 & 0.3 & \pm & 0.2 & - & - & - \\
\hline \hline
\end{tabular}

We perform a minimum $\chi^{2}$ fit of the above parametrization to the measured $Z p_{T}$ distribution. The MINUIT [57] function minimization programs are used. For each MINUIT variation of the parameters, a $\chi^{2}$ is calculated between the measured $Z p_{T}$ spectrum and the parametrization:

$$
\chi^{2}=\sum_{i, j}\left(X_{i}-P_{i}\right) \cdot \sigma_{i j}{ }^{-1} \cdot\left(X_{j}-P_{j}\right),
$$

where $X_{i}$ is the measured $d \sigma / d p_{T}, P_{i}$ is the value of the spectrum given by the parametrization for the $i^{\text {th }}$ bin, and $\sigma_{i j}{ }^{-1}$ is the inverse of the covariance matrix. The set of parameter values that minimizes the $\chi^{2}$ is the set of best fit parameters. The minimization yields a fit $\chi^{2}$ of 4 for 8 degrees of freedom (5 fit parameters for 13 data points). The values of the best fit parameters and their errors returned by MINUIT are summarized in Table 7.4. This table also includes the nominal fitted parameter values [58] for the $W$ spectrum.

\subsubsection{Comparison With QCD Prediction}

The results are also shown in Figure 7.1, and compared with the QCD next-to-leading order calculation.[8] The QCD prediction is plotted as a band; the width of the band indicates the uncertainty in the prediction. (See Section 1.3.) This prediction agrees with our result. 
Table 7.4: The fit parameters of parametrizations of the $W$ and the $Z p_{T}$ spectra.

\begin{tabular}{cll}
\hline Parameter & $W$ & $Z$ \\
\hline B & $1.434 \times 10^{-4}$ & $(2.450 \pm 1.931) \times 10^{-4}$ \\
C & -0.07 & $-0.259 \pm 0.166$ \\
D & -13.8 & $-9.611 \pm 4.152$ \\
E & $3.42 \times 10^{-2}$ & $(2.411 \pm 1.114) \times 10^{-2}$ \\
F & 3.09 & $2.483 \pm 0.675$ \\
\hline
\end{tabular}

The results obtained separately for the $Z \rightarrow e e$ and $Z \rightarrow \mu \mu$ samples also agree with the QCD prediction, and with each other. These are shown in Figure 7.2.

To make the comparison more quantitative, we calculate a $\chi^{2}$ between our result, from the combined $Z \rightarrow e e$ and $Z \rightarrow \mu \mu$ samples, and the nominal QCD prediction, taken as the median of the band in Figure 7.1. The $\chi^{2}$ is obtained using the covariance matrix $\sigma_{i j}$ as in Equation 7.11 with $P_{i}$ given by QCD predictions. We obtain a $\chi^{2}$ of 5 for 13 degrees of freedom (no arbitrary normalization factors). But because of the large uncertainty in the QCD calculation, we cannot treat this as a precise test of the predictions. For example, we cannot use a $\chi^{2}$ test of this type to determine the $Q^{2}$ scale because there are also large uncertainties in the parametrization of the parton momentum distributions, and higher order corrections, etc.. For example, a bad $\chi^{2}$ for a particular choice of the $Q^{2}$ could be due to problems in the parton distribution parametrization.

\subsubsection{Comparison With the $W p_{T}$ Distribution}

Since the $W$ and the $Z$ are both electroweak vector bosons, we expect their production properties, in particular their $p_{T}$ distributions, to have similar characteristics. The $W$ total production cross section in pip collisions at $\sqrt{s}=1.8 \mathrm{TeV}$ is approximately three times that for the $Z$. This can be understood as follows, using estimates based on the dominant leading order boson production process. The cross sections can be expressed in terms of the boson-quark couplings and the structure functions.[59] For simplicity, we only consider 


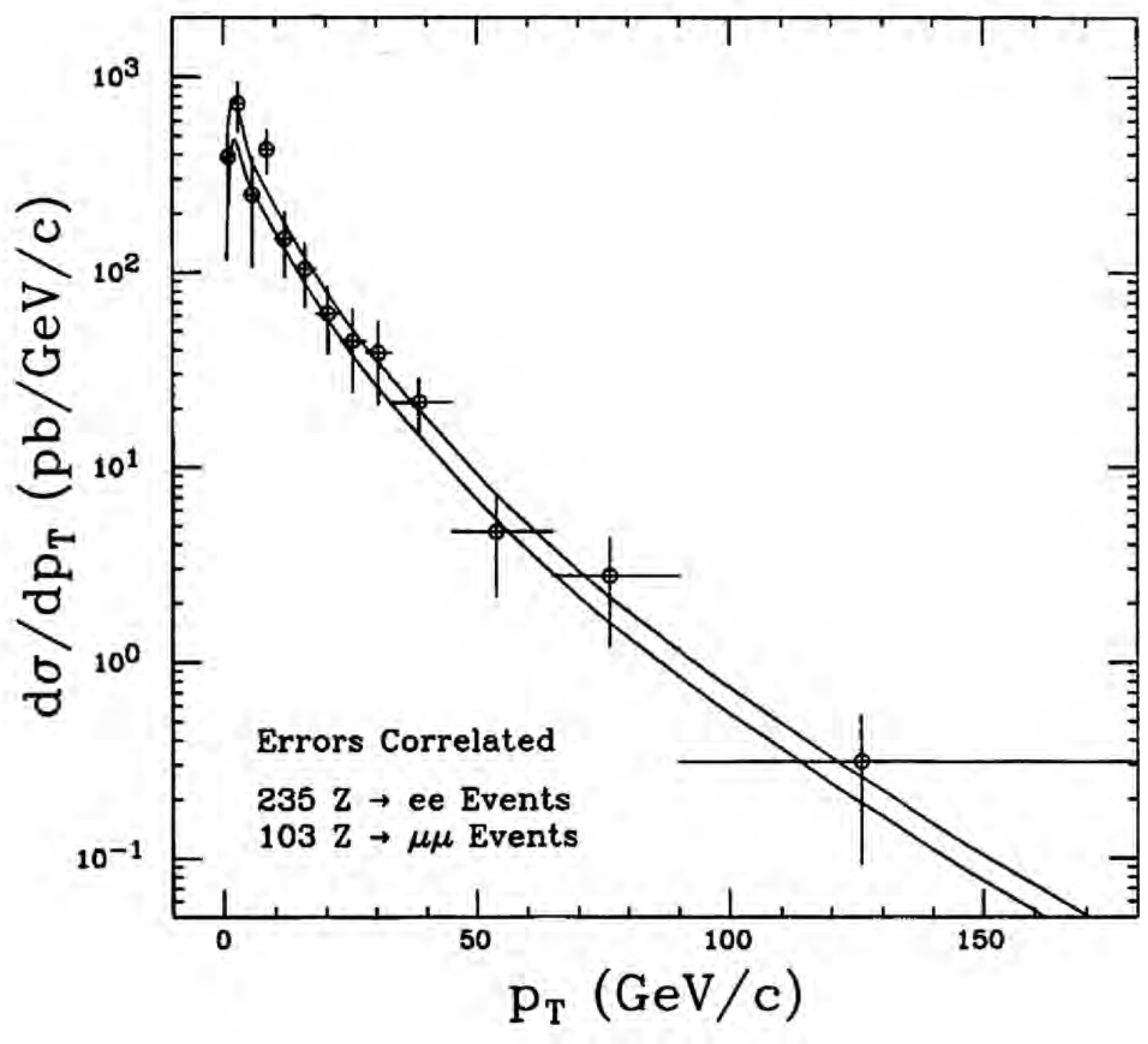

Figure 7.1: The differential cross section for $Z$ production for the combined $Z \rightarrow e e$ and $Z \rightarrow \mu \mu$ samples. The next-to-leading order QCD calculation is shown as a band; the width of the band indicates the theoretical uncertainties. 


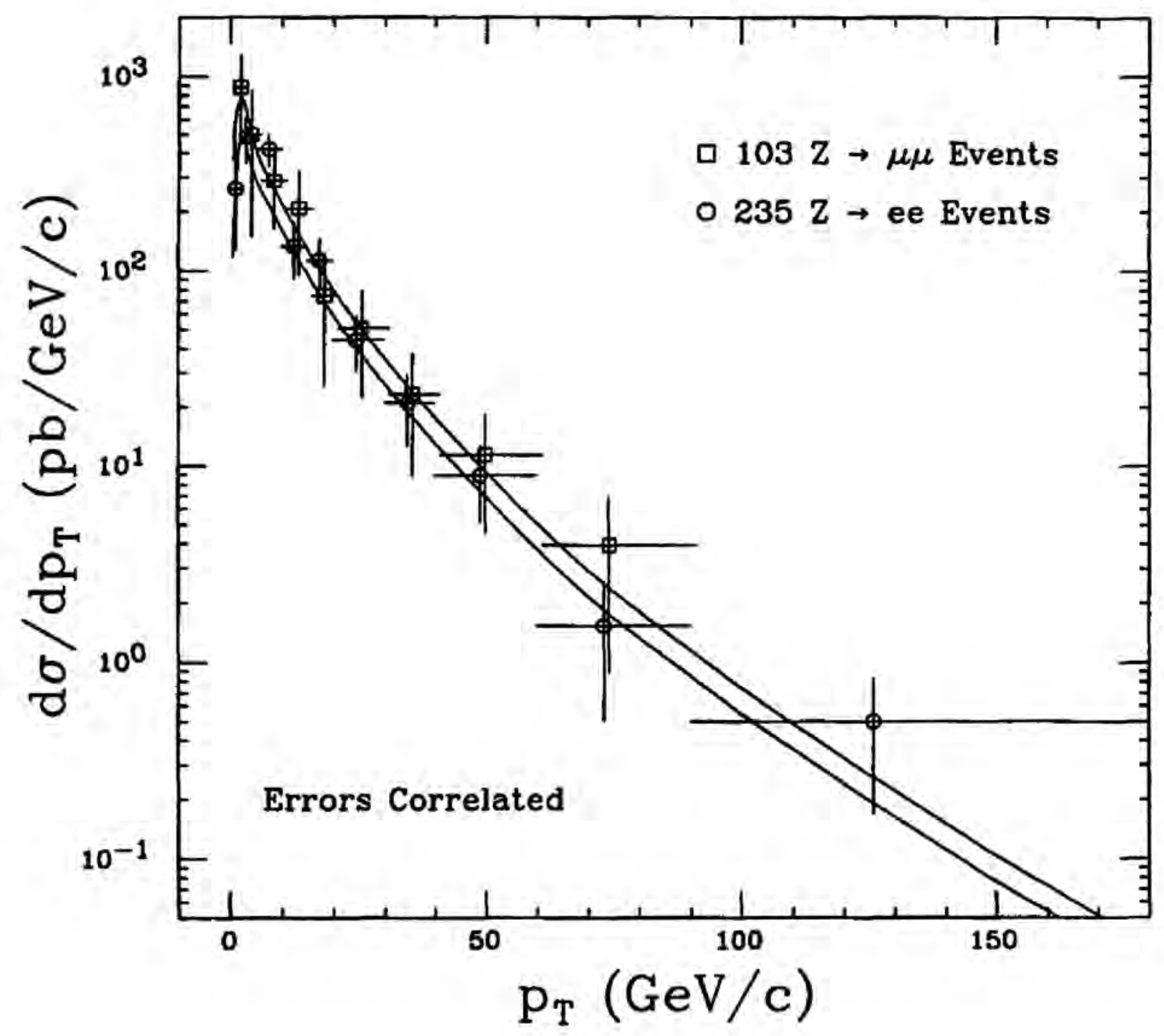

Figure 7.2: The differential cross section for $Z$ production for the $Z \rightarrow e e$ and $Z \rightarrow \mu \mu$ samples separately. The next-to-leading order QCD calculation is shown as a band; the width of the band indicates the theoretical uncertainties. 
production by valence quarks. The ratio of the cross sections is

$$
\frac{\sigma W}{\sigma Z} \approx 2 \times \frac{u \bar{d}+d \bar{u}}{0.6 u \bar{u}+0.7 d \bar{d}}
$$

where the factors of 0.6 and 0.7 come from the boson-quark couplings, and $u$ and $d$ are the parton distributions. Furthermore, let the boson rapidity $y=0$, then the fraction of the proton momentum carried by the colliding quarks is $x=M / \sqrt{s} \approx 0.04$. At this $\mathrm{x}$ value, the EHLQ structure function parametrization yields $u \approx 2 d$. The result $\sigma_{W} / \sigma_{Z} \approx 3$ follows.

The shapes of the $W$ and $Z p_{T}$ distributions are also expected to be slightly different,[60] mainly due to the following two sources:

- The $W, Z$ masses are different and the dependence of the production cross section on the boson mass is different at low and high $p_{T}$. For $\bar{p} p \rightarrow$ boson + parton, the $x$ 's of the structure functions can be expressed as: ${ }^{1}$

$$
\begin{aligned}
& x_{a}=\sqrt{\frac{p_{T}^{2}}{s}}\left[e^{y_{q}}+\sqrt{1+\left(\frac{M}{p_{T}}\right)^{2}} e^{y w}\right], \\
& x_{b}=\sqrt{\frac{p_{T}^{2}}{s}}\left[e^{-y_{\varphi}}+\sqrt{1+\left(\frac{M}{p_{T}}\right)^{2}} e^{-y w}\right],
\end{aligned}
$$

where $y_{q}\left(y_{W}\right)$ is the rapidity of the parton (boson) produced, and $M$ is the boson mass. For simplicity, set $y_{g}=0$ and $y_{W}=0$ to obtain

$$
x_{a}=x_{b}=\sqrt{\frac{p_{T}^{2}}{s}}+\sqrt{\frac{M^{2}+p_{T}^{2}}{s}} .
$$

So at low $p_{T}, x$ is roughly $\sqrt{M^{2} / s}$; whereas at high $p_{T}, x$ goes like $\sqrt{p_{T}^{2} / s}$. Thus, there is a difference between the ratio of the $W$ to $Z$ cross sections at low $p_{T}$ and the ratio at high $p_{T}$. That is, the $W$ and $Z$ mass difference leads to a difference in the shapes of the $p_{T}$ spectra.

- At high center of mass, $\sqrt{s}=1.8 \mathrm{TeV}$, the bosons at low $p_{T}$ are primarily produced by gluons and sea quarks, and so the $W$ and the $Z$ probe the same structure functions.

\footnotetext{
${ }^{1}$ These can be easily derived from energy and momentum conservation, and using the expression $\left(\sqrt{M^{2}+p_{T}^{2}} \cosh y, 0, p_{T}, \sqrt{M^{2}+p_{T}^{2}} \sinh y\right)$ for the four-vector.
} 
At large $p_{T}$, higher $x, W, Z$ production by the valence quarks dominates. Since the couplings of the $Z$ to $u \bar{u}$ and $d \bar{d}$ are different from that of the $W$ to $u \bar{d}$ and $\bar{u} d$, there is also a difference in the shape of the $d \sigma / d p_{T}$ for the $W \mathrm{~s}$ and the $Z$.

Note that the first effect is dominant at low $p_{T}$, while the second effect is dominant at high $p_{T}$. It is nevertheless interesting to compare the shapes of the $W$ and the $Z p_{T}$ distributions. We calculate a $\chi^{2}$ between the measured $Z p_{T}$ distribution and a parametrization of the $W$ $p_{T}$ distribution given by Equation 7.10 with the nominal parameters shown in Table 7.4. The $\chi^{2}$ is calculated using the covariance matrix $\sigma_{i j}$, as in Equation 7.11, and thus accounts for the correlation in the uncertainties among the bins in the measured $Z p_{T}$ distribution. But it does not account for error in the $W p_{T}$ spectrum parametrization. We obtain a $\chi^{2}$ of 12 for 12 degrees of freedom, with an arbitrary overall normalization factor, indicating good agreement between the shapes of the the $W$ and the $Z p_{T}$ spectra.

In Figure 7.3, we plot the following:

1. The ratio of the measured $Z p_{T}$ distribution to the nominal QCD prediction.

2. The expectation from QCD for the above ratio, shown as a band to indicate the theoretical uncertainty in the nominal QCD prediction for the $Z p_{T}$ distribution.

3. The ratio of the measured $W p_{T}$ distribution [61] to the nominal QCD prediction for the $Z p_{T}$ distribution. It is normalized by the factor of 3.12 of the measured ratio of the $W$ and $Z$ total cross sections[31].

4. The ratio of the QCD prediction for the $W p_{T}$ distribution to that for the $Z p_{T}$ distribution. It is normalized by the ratio of the measured $W$ and $Z$ total cross sections, as in the previous item.

Within the measurement uncertainties, the $W$ and the $Z p_{T}$ spectra have the same shape. This plot also shows the agreement of the $Z p_{r}$ distribution measurement with the corresponding QCD prediction on a linear scale. 


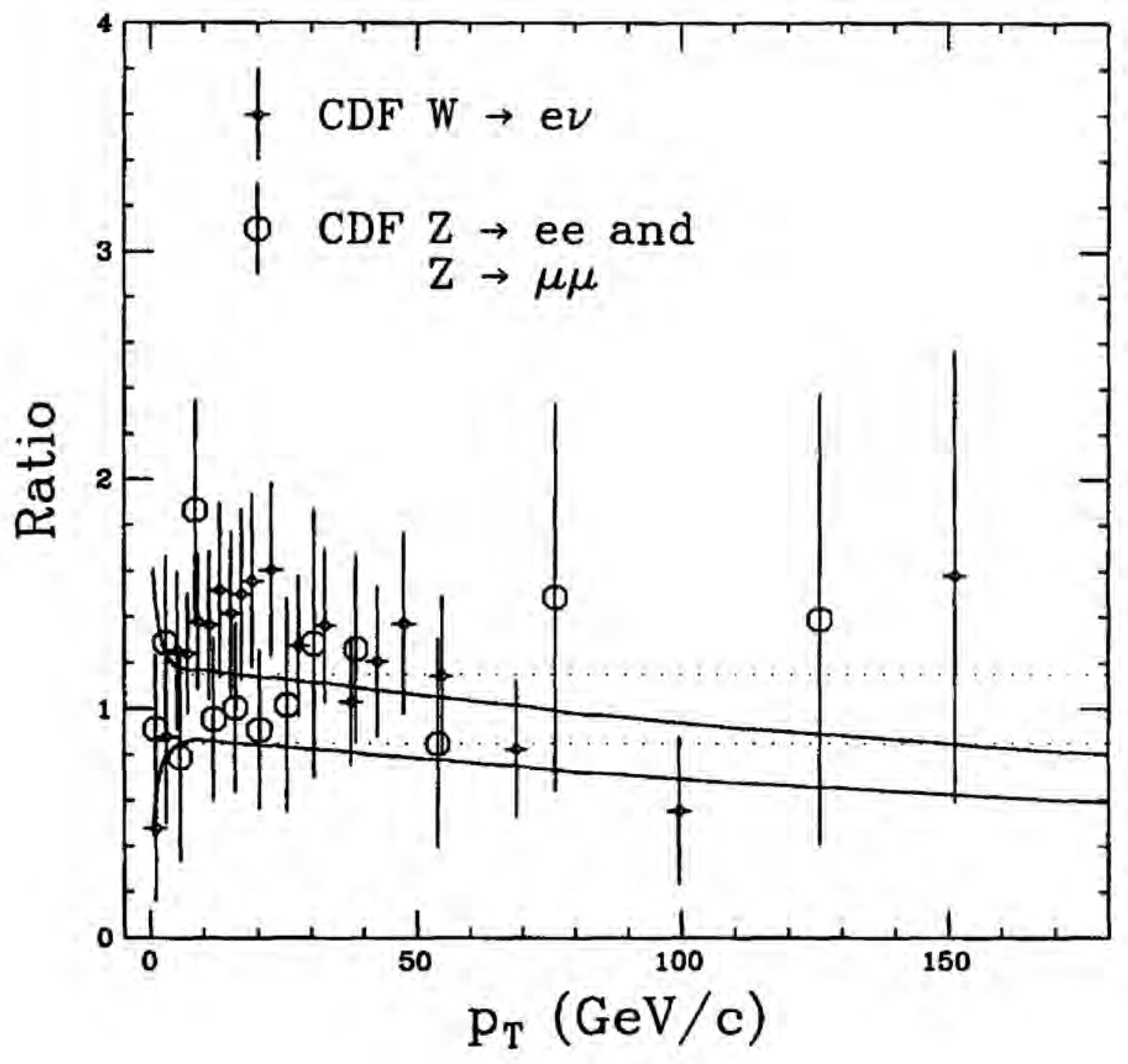

Figure 7.3: The ratio of the $W$ and $Z p_{T}$ spectra to the nominal QCD prediction for the $Z$; the $W$ 's are normalized by the ratio of the measured $W$ and $Z$ total cross sections. The QCD prediction is shown as a band of solid (dotted) lines for the $W(Z)$. 


\subsection{Conclusion}

In summary, we have measured the $Z$ differential cross section as a function of $p_{T}$. The resulting $d \sigma / d p_{T}$, corrected for acceptance and resolution smearing effects, is in good agreement with a next-to-leading order QCD calculation, in both the shape and the absolute normalization, over the range $0<p_{T}<160 \mathrm{GeV} / c$ available from this data sample.

The uncertainties in the measurement are dominated by the statistical uncertainties. These will be substantially reduced in the future with more data collected at the CDF experiment. To make a quantitative test of $\mathrm{QCD}$, however, the theoretical uncertainty must also be reduced. That will require, among other improvements, the calculation of higher order diagrams and improved parton distribution parametrizations. With more data and progress on the theoretical front, the $Z p_{T}$ distribution will lead to a better understanding of the nature of the strong interactions. 


\section{Bibliography}

[1] F.J. Hasert et. al., Phys. Lett. 46B, (1973)138; Nucl. Phys. B73, (1974)1;

A. Benvenuti et. al., Phys. Rev. Lett. 32, (1974)800.

[2] S.L. Glashow, Nucl, Phys. B22, (1961)579;

A. Salam in Proc. 8th Nobel Symposium, p.367, ed. N.Svertholm (Almqvist and Wik-

sell, Stockholm,1968);

S.Weinberg, Phys, Rev. Lett. 19, (1967)1264;

S.L.Glashow, J.Iliopoulos, and L.Maiani, Phys. Rev. D2, (1970)1285;

G.'t Hooft, Nucl. Phys. B33, (1971)173 and B35, (1971)167.

[3] UA1 Collaboration, G.Arnison et. al., Phys. Lett. 122B, (1983)103;

UA2 Collaboration, M.Banner et. al., Phys. Lett. 122B, (1983)476;

UA1 Collaboration, G.Arnison et. al., Phys. Lett. 126B, (1983)398;

UA2 Collaboration, P.Bagnaia et. al., Phys. Lett. 129B, (1983)130.

[4] UA2 Collaboration, J.Alitti et. al., Phys. Lett. 241B, (1990)150;

CDF Collaboration, F.Abe et. al, Phys. Rev. Lett. 65, (1990)2243.

[5] CDF Collaboration, F.Abe et. al., Phys. Rev. Lett. 63, (1989)720;

Mark-II Collaboration, G.S.Abrams et, al., Phys. Rev, Lett. 63, (1989)724, 2173;

L3 Collaboration, B.Adeva et. al., Phys. Lett. B231, (1989)509;

ALEPH Collaboration, D.DeCamp et. al., phys. Lett. B231, (1989)519;

OPAL Collaboration, M.Akrawy et. al., phys. Lett. B231, (1989)530;

DELPHI Collaboration, P.Aarnio et. al., phys. Lett. B231, (1989)539. 
[6] H.Burkhardt and J.Steinberger, CERN-PPE/91-50, to be published in Vol. 41 of the Annu. Rev, of Nucl. Part. Sci.

[7] A recent review of experimental tests of QCD can be found in G.Altarelli, Annu. Rev. Nucl. Part. Sci., 39, (1989)357.

[8] P. B. Arnold and R. P. Kauffman, Nucl. Phys. B349, (1991)381.

[9] Particle Data Group, Phys. Lett. 239B, (1990)

[10] P .B. Arnold, M. H. Reno, Nucl. Phys. B319, (1989)37;

[11] R. K. Ellis, G. Martinelli and R. Petronzio, Nucl. Phys. B211, (1983)106; R. Gonsalves, J. Pawlowski and C.-F. Wai, Phys. Rev. D40, (1989)2245.

[12] J. Collins, D. Soper and G. Sterman, Nucl. Phys. B250, (1985)199; G. Altarelli, R. K. Ellis, M. Greco and G. Martinelli, Nucl. Phys., B246, (1984)12; C.Davies, B.Webber and W. Stirling, Nucl. Phys. B256, (1985)413.

[13] P.N. Harriman, A.D. Martin, R.G. Roberts and W.J. Stirling, Phys. Rev. D42, (1990)798.

[14] UA2 Collaboration, J.Alitti et. al., Z. Phys. C47, (1990)523; UA1 Collaboration, C.Albajar et. al., Z. Phys. C44, (1989)15.

[15] J. Peoples, in Proc. of 8-th Topical Workshop on Proton-Antiproton Collider Physics, Castiglione, (1989).

[16] S. van der Meer, in Proc. of the SLAC and Fermilab Summer Schools on the Physics of Particle Accelerators, AIP Conference Proceedings 153, AIP, New York, 1987.

[17] CDF Collaboration, F. Abe et. al., Nucl. Instrum. Methods Phys. Res., Sect. A, 271, (1988)387.

[18] J. Konigsherg, private communication. Preliminary results from analysis of 1990 test beam data. 
[19] G.W. Foster et. al., Nucl. Instrum. Methods Phys. Res., Sect. A, 269, (1988)93.

[20] D. Brown, M. Franklin, CDF Notes 696,811 .

[21] Peter Berge, private communications.

[22] Aseet Mukherjee, "Alignment and Calibration of the CDF Central Detector", NIM article in preparation.

[23] C. Newman-Holmes, et. al., Nucl. Instrum. Methods Sect. A 274, (1989)443. There was a small leak in the cryogenic system and the operating current of the solenoid was lowered to prevent further damage during the run.

[24] T. Kamon: CDF Note 905; T.Kamon, D.Connor, S.Hahn:CDF Note 989.

[25] S. Kim, S. Ogawa: CDF Note 1021; S. Kim, et. al.:CDF Note 906.

[26] J. Ng: CDF Note 968 .

[27] E. Kearns: CDF Note 1077;

[28] F. Abe et. al., Phys. Rev. Lett. 62, (1989)613; F. Abe et. al., Phys. Rev. D43, (1991)664;

[29] C. Grosso-Pilcher and S. White, CDF Note 1202

[30] UA4 Collaboration, M. Bozzo et al., Phys. Lett. B147, (1984)392;

UA4 Collaboration, D. Bernard et al., Phys. Lett. B198, (1987)583.

[31] CDF Collaboration, F. Abe et. al., Phys. Rev. D. 44, (1991)29.

[32] E. Kearns, Ph.D. Thesis, Harvard University, June 1990. (Unpublished)

[33] P. Hurst, Ph.D. Thesis, University of nlinois at Urbana-Champaign, 1990. (Unpublished)

[34] A. Byon-Wagner et. al., CDF Note 1263. 
[35] J. Proudfoot, CDF Note 935.

[36] G. Ascoli, et. al., Nucl. Instrum. Methods Sect. A 268, (1988)33-40.

[37] D. Smith, Ph.D. Thesis, University of Illinois at Urbana-Champaign, December 1989. (Unpublished)

[38] H. Grassmann, T. Westhuing, CDF Note 1012; M.Gold, CDF Note 1229.

[39] A. Gauthier et. al.,CDF Notes 937, 1029, 1106,1145.

[40] 1. Hinchliffe, PAPAGENO, a QCD Monte Carlo, (in preparation); M. Shapiro and D. Brown: CDF Note 1221.

[41] F. Paige and S. D. Protopopescu, Isajet Monte Carlo version 6.22, BNL Report No. BNL38034, 1986 (unpublished).

[42] R. D. Field and R. P. Feynman, Nucl. Phys. B136, (1978)1.

[43] D. Brown, et. al., CDF Note 753.

[44] S. Behrends, CDF Note 1286.

[45] T. Hessing, et. al., CDF Note 1131; B. Winer, CDF Note 1239.

[46] D. Brown,B.Flaugher, CDF Note 1340.

[47] D. Brown, Ph.D. Thesis, Harvard University, June 1989. (Unpublished)

[48] Eichten, Hinchliffe, Lane, and Quigg: Rev. Mod. Phys. 58, (1986)1065.

[49] F.T. Brandt, G. Kramer, Su-Long Nyeo, Z. Phys. C48, (1990)301.

[50] F.A. Berends, W.T. Giele, H. Kuijf, R. Kleiss, W.J. Stirling, Phys. Lett. B224, (1989)237.

[51] Martin, Roberts, and Stirling: Phys. Rev. D37, (1988)1161. 
[52] M. Diemoz, F. Ferroni, E. Longo, and G. Martinelli: Z. Phys. C39, (1988)21.

[53] B. Winer, P. Tipton, CDF Notes 1287.

[54] M. Gold, CDF Note 913.

[55] M. Gold, A. Yagil, A. Clark, CDF Note 1094.

[56] A. B. Wicklund, private communications.

[57] F. James, M. Roos, CERN Computer Center Program Library Document, D506, 1989.

[58] B. Winer, private communications.

[59] See for example, V. Barger and R. Phillips, Collider Physics, Addison-Wesley, New York, 1988, Chapter 8 .

[60] Peter Arnold and Russel Kaufman, private communications.

[61] F. Abe et. al., Phys. Rev. Lett. 66, (1991)2951. 


\section{ProQuest Distribution Agrement}

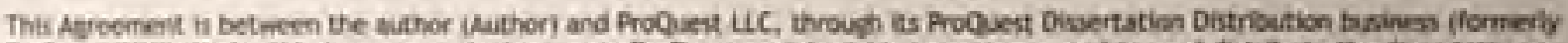

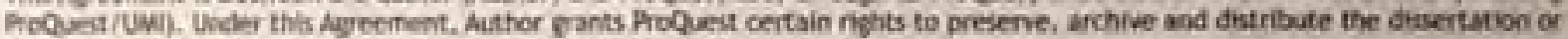
thesis gthe Worky, absuract, and index terms provided by Aughor to ProQuest.

\section{Section L Licene for Inclusion of the Work in ProQuest Publishing Program}

Grant of Rights. Author heveby grants to ProGuest the non-exclusive, worldwide right to noproduce, distribute, didplay and threment the Work ili whole or in part) in such tampble and electronic formats as may be in existerce now or developed in the futiare. Author further gants to Aroquat the right to incluibe the abstract, bituography and other eneludata in the Proquett Dissertations is Theses

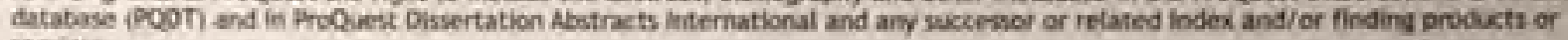
services.

Proquest Publishing Program - Election and Elements, The rights ganted above shail be exercised accordine to the publishilre

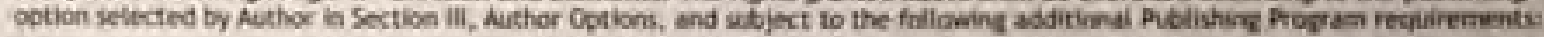

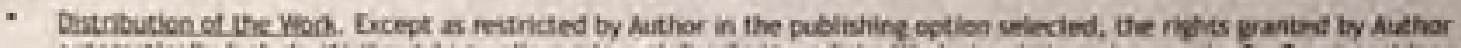
automatically include (1) bie right to allow sale and distribution of the Work, in whole or in part, by Anquest and its saies agents and dittributers and (2) the right to make the Abstract. bibliograghic dats and any meta duts askeclated with the Work avaluble ta seavch engines and harventers.

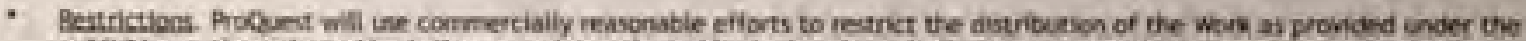
publishine option selested by Ruthor or as later piected by duthor through direct contact with Proquest. Such election is wabject to Aethor's Dewee Grantine institution Directives. With respect to restrictiona requested after subminskin of the Wherk.

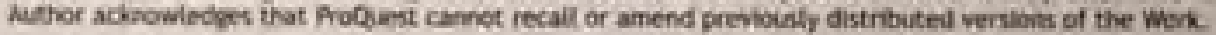

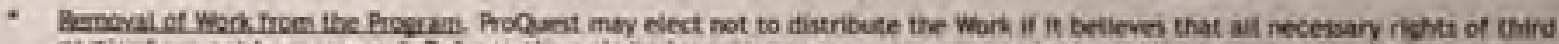
parties have not bem secured. Aeles to the website hetp://nwweproquest.com/products uni/distertations/for information

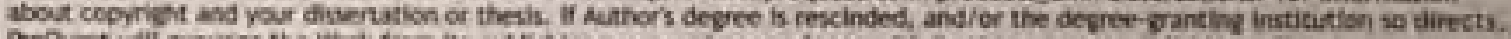
Proquest will expange the Work from its publishing program is accordance with its then current publishing policie.

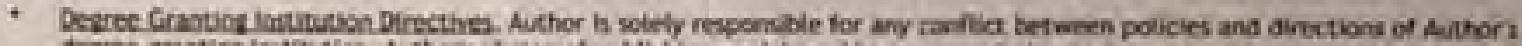

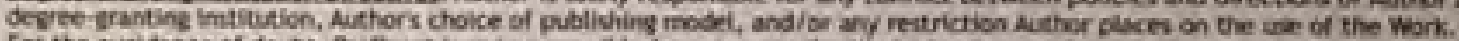

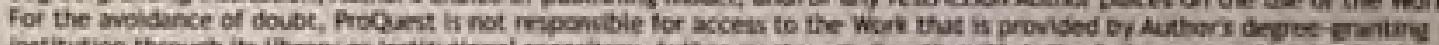

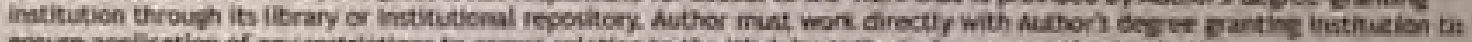

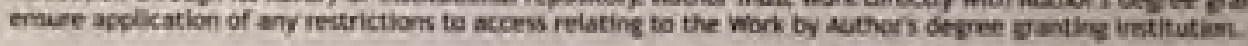

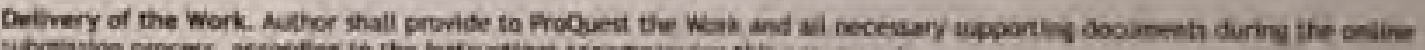

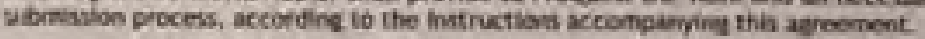

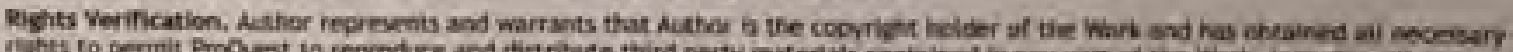

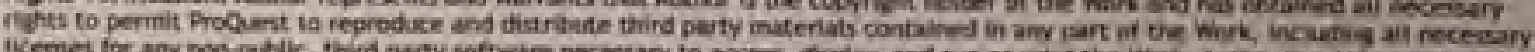

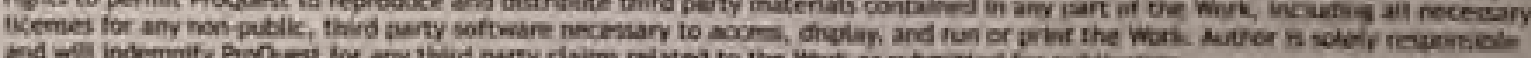

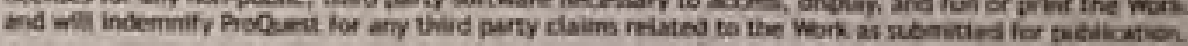

Ocan Access Aublitahine Pina

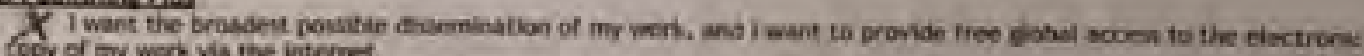

Coy of ay work vis the interret.

$X$ Iimbentast that i wili not te eliectie to nexehe ravaitin.

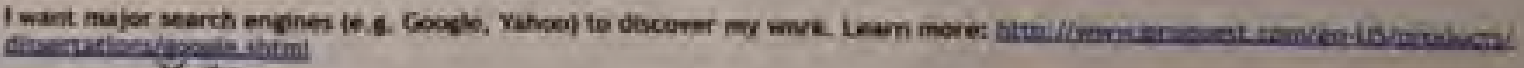

$$
\text { Xin }
$$

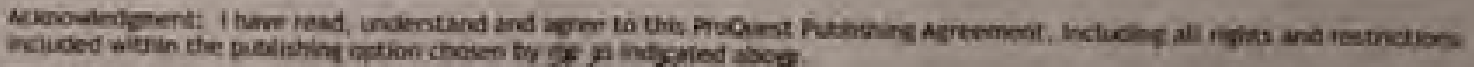

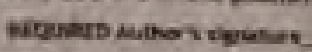
Prive itorne) 